\author{
Universidade de São Paulo \\ Instituto de Física de São Carlos
}

Guylherme Emmanuel Tagliaferro de Queiroz

\title{
Processamento digital de sinais aplicado a análise de distribuição de tempos de relaxação em sinais de ressonância magnética nuclear.
}



Guylherme Emmanuel Tagliaferro de Queiroz

\section{Processamento digital de sinais aplicado a análise de distribuição de tempos de relaxação em sinais de ressonância magnética nuclear.}

Dissertação apresentada ao Programa de PósGraduação em Física do Instituto de Física de São Carlos da Universidade de São Paulo, para obtenção do título de mestre em Ciências.

Área de Concentração: Física Aplicada - Opção Física Computacional

Orientador: Prof. Dr. Rodrigo Capobianco Guido

\section{Versão Corrigida}

(versão original disponível na Unidade que aloja o Programa) 
AUTORIZO A REPRODUÇÃO E DIVULGAÇÃO TOTAL OU PARCIAL DESTE TRABALHO, POR QUALQÜER MEIO CONVENCIONAL OU ELETRÔNICO PARA FINS DE ESTUDO E PESQUISA, DESDE QUE CITADA A FONTE.

Ficha catalográfica elaborada pelo Serviço de Biblioteca e Informação do IFSC, com os dados fornecidos pelo(a) autor(a)

Queiroz, G. E. T.

Processamento digital de sinais aplicado a análise de distribuição de tempos de relaxação em sinais de ressonância magnética nuclear / G. E. T. Queiroz; orientador Rodrigo Capobianco Guido versão corrigida -- São Carlos, 2015. $155 \mathrm{p}$.

Dissertação (Mestrado - Programa de Pós-Graduação em Física Aplicada Computacional) -- Instituto de Física de São Carlos, Universidade de São Paulo, 2015.

1. Processamento digital de sinais. 2. Ressonância magnética nuclear. 3. Equações integrais de Fredholm. 4. Meios porosos. I. Capobianco Guido, Rodrigo, orient. II. Título. 


\section{AGRADECIMENTOS}

Este trabalho, em primeiro lugar, é dedicado a todos aqueles que depositaram sua crença em mim. Não existe nada que quantifique tamanha gratidão que tenho por aqueles que me acompanham ou me acompanharam de alguma forma. Ao meus pais, a gratidão eterna pelo amor que jamais faltou e me permitiu chegar onde estou hoje. Ao meu ex-orientador da graduação Prof. Dr. Luiz Antônio Bássora, embora a vida, por hora, nos tenha imposto caminhos paralelos, esteja certo do imenso prazer que tenho de lhe chamar de amigo, obrigado pelo conhecimento que me foi passado e pelas portas que me abriu. Ao meu orientador Prof. Dr. Rodrigo Capobianco Guido, serei eternamente grato pela dedicação e paciência. Sou grato também a CAPES por fornecer o auxilio que me permitiu realizar este trabalho. 

Não adentre à boa noite tão gentilmente assim, É necessário delirar ao fim do dia,

Lute, lute pela luz que chega ao fim,

(..)

Dylan Thomas. 



\section{RESUMO}

QUEIROZ, G. E. T. Processamento digital de sinais aplicado a análise de distribuição de tempos de relaxação em sinais de ressonância magnética nuclear. 2015. 155 p. Dissertação (Mestrado em Ciências) - Instituto de Física de São Carlos, Universidade de São Paulo, São Carlos, 2015.

Sabe-se que a relaxação de líquidos em meios porosos envolve três mecanismos principais: relaxação bulk, relaxação de superfície e difusão. Muitas vezes, os processos de relaxação de líquidos confinados em meios porosos são dominados pelo processo de relaxação de superfície e difusão do fluído. No chamado regime de difusão rápida, a relaxação de um único poro é comandada por uma função mono exponencial que depende, principalmente, da relação superfície-volume do poro, de modo que em um material poroso, isto é, contendo uma distribuição ampla de tamanho de poros, o sinal de decaimento de magnetização obtido por meio da ressonância magnética nuclear é formado pela soma de exponenciais com diferentes tempos de relaxação. O problema-chave abordado neste trabalho consiste, portanto, em obter por meio desse sinal de magnetização a distribuição dos tempos de relaxação que controlam o decaimento das funções mono-exponenciais. Matematicamente, esse sinal de decaimento de magnetização pode ser descrito na forma geral de uma equação integral de Fredholm do primeiro tipo, cuja solução é um reconhecido problema inverso mal-posto. As abordagens utilizadas na tentativa de solucionar o problema são oriundas de uma área conhecida como processamento digital de sinais, e os seguintes métodos são analisados e comparados neste trabalho: algoritmo dos mínimos quadrados médios com restrição de não negatividade (LMS-NN), algoritmo dos mínimos quadrados médios com restrição de não negatividade e regularizado (LMS-RNN), redes recorrentes de Hopfield e o já bem conhecido na solução de problemas inversos mal-postos, o algoritmo dos mínimos quadrados regularizado (LS-R). Os resultados obtidos no trabalho são bastante positivos, demonstrando que, além do LS-R, existem outras alternativas na solução do problema, que principalmente, permitem atestar as soluções obtidas por qualquer um dos algoritmos.

Palavras-chave: Processamento digital de sinais. Ressonância magnética nuclear. Equações integrais de Fredholm. Meios porosos. 



\section{ABSTRACT}

QUEIROZ, G. E. T. Digital signal processing applied to relaxation times distribution analysis in nuclear magnetic resonance signals. 2015. 155 p. Dissertação (Mestrado em Ciências) Instituto de Física de São Carlos, Universidade de São Paulo, São Carlos, 2015.

It is known that the relaxation of liquids in porous media involves three principal mechanisms: bulk relaxation, surface relaxation, and diffusion. Relaxation processes of confined fluids in porous media are often controlled by surface relaxation process and diffusion. In the so-called fast diffusion regime, the relaxation of a single pore is governed by a mono-exponential function that depends primarily on the relation surface-volume of the pore, so that in a porous medium, i.e, in a medium which contains a wide distribution of pore sizes, the signal of magnetization decay obtained by nuclear magnetic resonance is composed by a sum of exponentials controlled by different relaxation times. The main issue discussed in this work consists in obtaining the distribution of relaxation times that controls the decay of the mono-exponential functions that comprise the magnetization signal. Mathematically this signal of magnetization decay can be generally described as a Fredholm integral equation of the first kind, whose solution is a recognized ill-posed inverse problem. The approaches adopted to try to solve the problem come from an area known as digital signal processing, and the following methods analyzed and compared are: non-negative least mean square algorithm (NN-LMS), regularized and nonnegative least mean square algorithm (RNN-LMS), recurrent Hopfield networks and regularized least square algorithm (R-LS), acknowledged in the solution of ill-posed inverse problems. The results obtained are very positive, and show that in addition to R-LS there are other alternatives in the solution of the problem, which mainly allow to attest the results achieved through any of the algorithms.

Keywords: Digital signal processing. Nuclear magnetic ressonance. Fredholm integral equations. Porous media. 



\section{LISTA DE FIGURAS}

Figura 2.1 - Diagrama do movimento de precessão do spin nuclear sujeito a um campo magnético externo $\mathbf{B}_{0} \ldots \ldots \ldots$. . . . . . . . . . . . . p.30

Figura 2.2 - Diagrama de um sistema com dois níveis de energia. . . . . . . . . . p.31

Figura 2.3 - Free Induction Decay. . . . . . . . . . . . . . . . . . . p. 33

Figura 2.4 - Sequência de inversão-recuperação, utilizada na medida de $T_{1} \ldots$. . . p.34

Figura 2.5 - Sequência de saturação-recuperação, utilizada na medida de $T_{1}$. . . p.35

Figura 2.6 - Refocalização com pulso de $180^{\circ}$, utilizado na medida de $T_{2} \ldots \ldots$. . . 36

Figura 2.7 - Fenômeno spin-eco, utilizado na medida de $T_{2} \ldots \ldots \ldots$ p. 36

Figura 2.8 - Sequência de pulsos na medida de $T_{2}$ proposta por Carl, Purcell, Meiboom e Gill. . . . . . . . . . . . . . . . . . . . . . . . . p. 37

Figura 2.9 - Possíveis mecanimos de relaxação no interior de um poro. . . . . . . p. 38

Figura 2.10 - Representação da relação entre tempos de relaxação e tamanho do poro. p. 39

Figura 2.11 - Forma geral do combinador linear adaptativo de múltiplas entradas . . p.42

Figura 2.12 - Diagrama do processo adaptativo do combinador linear envolvendo o sinal de saída $y_{k}$ e o sinal desejado $d_{k} \ldots \ldots$. . . . . . . . . p.43

Figura 2.13 - Superfície de desempenho quadrática bi-dimensional. . . . . . . . . p. 44

Figura 2.14 - Curvas de contorno da superfície de desempenho com a localização de $\mathbf{w}^{0} \ldots \ldots \ldots \ldots \ldots \ldots \ldots$. . . . . . . . . . . . . . . . . . . .

Figura 2.15 - Distribuição multi-log-normal composta por 4096 amostras logaritmomanicamente espaçadas no intervalo $\left[10^{-5}, 10^{-2}\right]$, com $A_{1}=0,8 \mathrm{e}$ $A_{2}=0,2, T_{c_{1}}=10^{-3}$ e $T_{c_{2}}=10^{-1}$ e $\sigma_{1}=2,5$ e $\sigma_{2}=1 \ldots$. . .

Figura 2.16 - Decaimento multi exponencial composto por 1024 amostras, contaminadas com ruído gaussiano, para a distribuição da figura 2.15 , com diversos tempos de aquisição. 
Figura 2.17 - Distribuição log-normal composta por 4096 amostras logaritmomanicamente espaçadas no intervalo $\left[10^{-5}, 10^{-2}\right]$, com $A_{1}=0,8, T_{c_{1}}=10^{-2}$

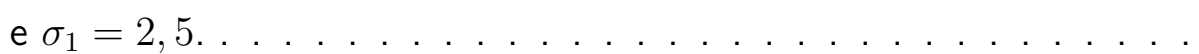

Figura 2.18 - Decaimento multi exponencial composto por 1024 amostras, contaminadas com ruído gaussiano, para a distribuição da figura 2.17, com diferentes tempos de eco.

Figura 2.19 - Unidades responsáveis pelo processamento nas redes neurais. a)Esquema simplificado de um neurônio biológico. b)Neurônio artificial típico . .

Figura 2.20 - Rede de Hopfield convencional.

Figura 2.21 - Gráfico da função logística. . . . . . . . . . . . . . . . . . . . .

Figura 2.22 - Representação geométrica da SVD para uma matriz $2 \times 2$. . . . . .

Figura 2.23 - Suavização da resposta (regularização) para diferentes valores de $\lambda$. .

Figura 3.1 - Interface do gerador de pares de sinais simulados desenvolvido. . . . . .

Figura 3.2 - Distribuição obtida através do algoritmo LMS-NN para o sinal simulado com $\tau^{*}=30 \mu$ s e comparado à distribuição simulada do teste um. . .

Figura 3.3 - Distribuição obtida através do algoritmo LMS-NN para o sinal simulado com $\tau^{*}=60 \mu \mathrm{s}$ e comparado à distribuição simulada do teste um. . .

Figura 3.4 - Distribuição obtida através do algoritmo LMS-NN para o sinal simulado com $\tau^{*}=120 \mu \mathrm{s}$ e comparado à distribuição simulada do teste um.

Figura 3.5 - Distribuição obtida através do algoritmo LMS-NN para o sinal simulado $\operatorname{com} \tau^{*}=240 \mu \mathrm{s}$ e comparado à distribuição simulada do teste um.

Figura 3.6 - Distribuição obtida através do algoritmo LMS-NN para o sinal simulado com $\tau^{*}=480 \mu s$ e comparado à distribuição simulada do teste um.

Figura 3.7 - Distribuição obtida através do algoritmo LMS-NN para o sinal simulado com $\tau^{*}=960 \mu \mathrm{s}$ e comparado à distribuição simulada do teste um.

Figura 3.8 - Distribuição obtida através do algoritmo LMS-NN combinando os sinais simulados com $\tau^{*}=30 \mu s, 60 \mu s, 120 \mu s, 240 \mu s, 480 \mu s$ e $960 \mu s$ e comparado à distribuição simulada para o teste um. 
Figura 3.9 - Distribuição obtida através do algoritmo LMS-RNN para o sinal simulado com $\tau^{*}=30 \mu \mathrm{s}$ e comparado à distribuição simulada do teste

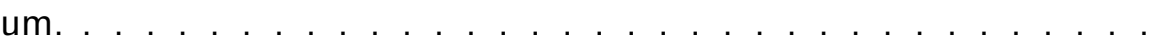

Figura 3.10 - Distribuição obtida através do algoritmo LMS-RNN para o sinal simulado com $\tau^{*}=60 \mu \mathrm{s}$ e comparado à distribuição simulada do teste um.

Figura 3.11 - Distribuição obtida através do algoritmo LMS-RNN para o sinal simulado com $\tau^{*}=120 \mu \mathrm{s}$ e comparado à distribuição simulada do teste um.

Figura 3.12 - Distribuição obtida através do algoritmo LMS-RNN para o sinal simulado com $\tau^{*}=240 \mu \mathrm{s}$ e comparado à distribuição simulada do teste um.

Figura 3.13 - Distribuição obtida através do algoritmo LMS-RNN para o sinal simulado com $\tau^{*}=480 \mu \mathrm{s}$ e comparado à distribuição simulada do teste um.

Figura 3.14 - Distribuição obtida através do algoritmo LMS-RNN para o sinal simulado com $\tau^{*}=960 \mu \mathrm{s}$ e comparado à distribuição simulada do teste um.

Figura 3.15 - Distribuição obtida através do algoritmo LMS-RNN combinando os sinais simulados com $\tau^{*}=30 \mu \mathrm{s}, 60 \mu \mathrm{s}, 120 \mu \mathrm{s}, 240 \mu \mathrm{s}, 480 \mu \mathrm{s}$ e $960 \mu \mathrm{s}$ e comparado à distribuição simulada para o teste um.

Figura 3.16 - Distribuição obtida através do algoritmo da rede recorrente de Hopfield para o sinal simulado com $\tau^{*}=30 \mu \mathrm{s}$ e comparado à distribuição simulada do teste um.

Figura 3.17 - Distribuição obtida através do algoritmo da rede recorrente de Hopfield para o sinal simulado $\operatorname{com} \tau^{*}=60 \mu \mathrm{s}$ e comparado à distribuição simulada do teste um.

Figura 3.18 - Distribuição obtida através do algoritmo da rede recorrente de Hopfield para o sinal simulado com $\tau^{*}=120 \mu \mathrm{s}$ e comparado à distribuição simulada do teste um. 
Figura 3.19 - Distribuição obtida através do algoritmo da rede recorrente de Hopfield para o sinal simulado com $\tau^{*}=240 \mu s$ e comparado à distribuição simulada do teste um. . . . . . . . . . . . . . . . . . . . .

Figura 3.20 - Distribuição obtida através do algoritmo da rede recorrente de Hopfield para o sinal simulado com $\tau^{*}=480 \mu s$ e comparado à distribuição simulada do teste um.

Figura 3.21 - Distribuição obtida através do algoritmo da rede recorrente de Hopfield para o sinal simulado com $\tau^{*}=960 \mu s$ e comparado à distribuição simulada do teste um.

Figura 3.22 - Distribuição obtida através do algoritmo LS-R para o sinal simulado com $\tau^{*}=30 \mu s$ e comparado à distribuição simulada do teste um. . .

Figura 3.23 - Distribuição obtida através do algoritmo LS-R para o sinal simulado $\operatorname{com} \tau^{*}=60 \mu \mathrm{s}$ e comparado à distribuição simulada do teste um. . .

Figura 3.24 - Distribuição obtida através do algoritmo LS-R para o sinal simulado com $\tau^{*}=120 \mu \mathrm{s}$ e comparado à distribuição simulada do teste um.

Figura 3.25 - Distribuição obtida através do algoritmo LS-R para o sinal simulado com $\tau^{*}=240 \mu s$ e comparado à distribuição simulada do teste um.

Figura 3.26 - Distribuição obtida através do algoritmo LS-R para o sinal simulado com $\tau^{*}=480 \mu s$ e comparado à distribuição simulada do teste um.

Figura 3.27 - Distribuição obtida através do algoritmo LS-R para o sinal simulado com $\tau^{*}=960 \mu \mathrm{s}$ e comparado à distribuição simulada do teste um.

Figura 3.28 - Distribuição obtida através do algoritmo LMS-NN para o sinal simulado $\operatorname{com} \tau^{*}=30 \mu s$ e comparado à distribuição simulada do teste dois.

Figura 3.29 - Distribuição obtida através do algoritmo LMS-NN para o sinal simulado com $\tau^{*}=60 \mu \mathrm{s}$ e comparado à distribuição simulada do teste dois.

Figura 3.30 - Distribuição obtida através do algoritmo LMS-NN para o sinal simulado com $\tau^{*}=120 \mu \mathrm{s}$ e comparado à distribuição simulada do teste dois. .

Figura 3.31 - Distribuição obtida através do algoritmo LMS-NN para o sinal simulado com $\tau^{*}=240 \mu s$ e comparado à distribuição simulada do teste dois. .

Figura 3.32 - Distribuição obtida através do algoritmo LMS-NN para o sinal simulado com $\tau^{*}=480 \mu s$ e comparado à distribuição simulada do teste dois. . 
Figura 3.33 - Distribuição obtida através do algoritmo LMS-NN para o sinal simulado com $\tau^{*}=960 \mu$ s e comparado à distribuição simulada do teste dois.

Figura 3.34 - Distribuição obtida através do algoritmo LMS-NN combinando os sinais simulados com $\tau^{*}=30 \mu s, 60 \mu s, 120 \mu s, 240 \mu s, 480 \mu s$ e $960 \mu s$ e comparado à distribuição simulada.

Figura 3.35 - Distribuição obtida através do algoritmo LMS-RNN para o sinal simulado com $\tau^{*}=30 \mu \mathrm{s}$ e comparado à distribuição simulada do teste dois.

Figura 3.36 - Distribuição obtida através do algoritmo LMS-RNN para o sinal simulado com $\tau^{*}=60 \mu \mathrm{s}$ e comparado à distribuição simulada do teste dois.

Figura 3.37 - Distribuição obtida através do algoritmo LMS-RNN para o sinal simulado com $\tau^{*}=120 \mu \mathrm{s}$ e comparado à distribuição simulada do teste dois.

Figura 3.38 - Distribuição obtida através do algoritmo LMS-RNN para o sinal simulado com $\tau^{*}=240 \mu \mathrm{s}$ e comparado à distribuição simulada do teste dois.

Figura 3.39 - Distribuição obtida através do algoritmo LMS-RNN para o sinal simulado com $\tau^{*}=480 \mu \mathrm{s}$ e comparado à distribuição simulada do teste dois.

Figura 3.40 - Distribuição obtida através do algoritmo LMS-RNN para o sinal simulado com $\tau^{*}=960 \mu \mathrm{s}$ e comparado à distribuição simulada do teste dois.

Figura 3.41 - Distribuição obtida através do algoritmo LMS-RNN combinando os sinais simulados com $\tau^{*}=30 \mu s, 60 \mu s, 120 \mu s, 240 \mu s, 480 \mu s$ e $960 \mu s$ e comparado à distribuição simulada.

Figura 3.42 - Distribuição obtida através do algoritmo da rede recorrente de Hopfield para o sinal simulado com $\tau^{*}=30 \mu s$ e comparado à distribuição simulada do teste dois.

Figura 3.43 - Distribuição obtida através do algoritmo da rede recorrente de Hopfield para o sinal simulado com $\tau^{*}=60 \mu \mathrm{s}$ e comparado à distribuição simulada do teste dois. 
Figura 3.44 - Distribuição obtida através do algoritmo da rede recorrente de Hopfield para o sinal simulado com $\tau^{*}=120 \mu \mathrm{s}$ e comparado à distribuição simulada do teste dois. . . . . . . . . . . . . . . . . . . . . . p. 103

Figura 3.45 - Distribuição obtida através do algoritmo da rede recorrente de Hopfield para o sinal simulado com $\tau^{*}=240 \mu \mathrm{s}$ e comparado à distribuição simulada do teste dois. . . . . . . . . . . . . . . . . . . . . . . . . . p. 104

Figura 3.46 - Distribuição obtida através do algoritmo da rede recorrente de Hopfield para o sinal simulado com $\tau^{*}=480 \mu \mathrm{s}$ e comparado à distribuição simulada do teste dois. . . . . . . . . . . . . . . . . . . . . .

Figura 3.47 - Distribuição obtida através do algoritmo da rede recorrente de Hopfield para o sinal simulado com $\tau^{*}=960 \mu \mathrm{s}$ e comparado à distribuição simulada do teste dois. . . . . . . . . . . . . . . . . . . . . p. 105

Figura 3.48 - Distribuição obtida através do algoritmo LS-R para o sinal simulado com $\tau^{*}=30 \mu \mathrm{s}$ e comparado à distribuição simulada do teste dois. . p. 106

Figura 3.49 - Distribuição obtida através do algoritmo LS-R para o sinal simulado com $\tau^{*}=60 \mu \mathrm{s}$ e comparado à distribuição simulada do teste dois.

Figura 3.50 - Distribuição obtida através do algoritmo LS-R para o sinal simulado com $\tau^{*}=120 \mu \mathrm{s}$ e comparado à distribuição simulada do teste dois. . p. 107

Figura 3.51 - Distribuição obtida através do algoritmo LS-R para o sinal simulado com $\tau^{*}=240 \mu \mathrm{s}$ e comparado à distribuição simulada do teste dois. .

Figura 3.52 - Distribuição obtida através do algoritmo LS-R para o sinal simulado com $\tau^{*}=480 \mu \mathrm{s}$ e comparado à distribuição simulada do teste dois. . p. 108

Figura 3.53 - Distribuição obtida através do algoritmo LS-R para o sinal simulado com $\tau^{*}=960 \mu \mathrm{s}$ e comparado à distribuição simulada do teste dois. .

Figura 3.54 - Distribuição de valores obtidas através do algoritmo LMS-NN para $\tau^{*}=$ $15 \mu$ s comparado com a distribuição simulada.

Figura 3.55 - Distribuição de valores obtidas através do algoritmo LMS-RNN para $\tau^{*}=15 \mu s$ comparado com a distribuição simulada.

Figura 3.56 - Distribuição de valores obtidas através do algoritmo da rede recorrente de Hopfield para $\tau^{*}=15 \mu$ s comparado com a distribuição simulada. 
Figura 3.57 - Distribuição de valores obtidas através do algoritmo dos mínimos quadrados regularizado para $\tau^{*}=15 \mu \mathrm{s}$ comparado com a distribuição simulada.

Figura 3.58 - Distribuição de valores obtidas através do algoritmo LMS-RNN para sinal experimental real $\operatorname{com} \tau^{*}=240 \mu s$.

Figura 3.59 - Distribuição de valores obtidas através do algoritmo LMS-RNN para sinal experimental real $\operatorname{com} \tau^{*}=480 \mu s$.

Figura 3.60 - Distribuição de valores obtidas através do algoritmo LMS-NN para sinal experimental real com $\tau^{*}=960 \mu s$.

Figura 3.61 - Distribuição de valores obtida através do algoritmo LMS-NN combinando os sinais experimentais reais com $\tau^{*}=240 \mu \mathrm{s}, 480 \mu \mathrm{s}$ e $960 \mu \mathrm{s}$.

Figura 3.62 - Distribuição de valores obtidas através do algoritmo LMS-RNN para sinal experimental real com $\tau^{*}=240 \mu s$.

Figura 3.63 - Distribuição de valores obtidas através do algoritmo LMS-RNN para sinal experimental real com $\tau^{*}=480 \mu s$.

Figura 3.64 - Distribuição de valores obtidas através do algoritmo LMS-RNN para sinal experimental real com $\tau^{*}=960 \mu s$.

Figura 3.65 - Distribuição de valores obtida através do algoritmo LMS-RNN combinando os sinais experimentais reais com $\tau^{*}=240 \mu \mathrm{s}, 480 \mu \mathrm{s}$ e $960 \mu \mathrm{s}$.

Figura 3.66 - Distribuição de valores obtidas através do algoritmo da rede recorrente de Hopfield para sinal experimental real com $\tau^{*}=240 \mu \mathrm{s}$.

Figura 3.67 - Distribuição de valores obtidas através do algoritmo da rede recorrente de Hopfield para sinal experimental real com $\tau^{*}=480 \mu \mathrm{s}$.

Figura 3.68 - Distribuição de valores obtidas através do algoritmo da rede recorrente de Hopfield para sinal experimental real com $\tau^{*}=960 \mu \mathrm{s}$.

Figura 3.69 - Distribuição de valores obtidas através do algoritmo mínimos quadrados regularizado para sinal experimental real com $\tau^{*}=240 \mu s$.

Figura 3.70 - Distribuição de valores obtidas através do algoritmo mínimos quadrados regularizado para sinal experimental real com $\tau^{*}=480 \mu \mathrm{s}$.

Figura 3.71 - Distribuição de valores obtidas através do algoritmo mínimos quadrados regularizado para sinal experimental real com $\tau^{*}=960 \mu s$. 



\section{LISTA DE TABELAS}

Tabela 3.1 - Parâmetros utilizados e resultados obtidos com algoritmos LMS-NN. . p.78

Tabela 3.2 - Parâmetros utilizados e resultados obtidos com algoritmos LMS-RNN. p. 83

Tabela 3.3 - Parâmetros utilizados e resultados obtidos com algoritmo de Hopfield. p. 87

Tabela 3.4 - Parâmetros utilizados e resultados obtidos com algoritmos LS-R. . . . p. p1

Tabela 3.5 - Comparativo entre os melhores resultados obtidos com cada algoritmo no primeiro teste. . . . . . . . . . . . . . . . . p. 92

Tabela 3.6 - Parâmetros utilizados e resultados obtidos com algoritmos LMS-NN para o teste dois. . . . . . . . . . . . . . . . . . p.96

Tabela 3.7 - Parâmetros utilizados e resultados obtidos com algoritmos LMS-RNN. p. 101

Tabela 3.8 - Parâmetros utilizados e resultados obtidos com algoritmo da rede recorrente de Hopfield para o teste dois. . . . . . . . . . . . . . p. 105

Tabela 3.9 - Parâmetros utilizados e resultados obtidos com algoritmos LS-R. . . . p. 109

Tabela 3.10 - Comparativo entre os melhores resultados obtidos com cada algoritmo para o teste dois. . . . . . . . . . . . . . . . . . p. . 110

Tabela 3.11 - Parâmetros utilizados e resultados obtidos para cada algoritmo no teste três. . . . . . . . . . . . . . . . . . . . . p. 113

Tabela 3.12 - Parâmetros utilizados e resultados obtidos com algoritmo de LMS-NN para o teste com sinal experimental real. . . . . . . . . . . . . . . p. 114

Tabela 3.13 - Parâmetros utilizados e resultados obtidos com algoritmos LMS-RNN para o teste com sinal experimental real. . . . . . . . . . . . p. 117

Tabela 3.14 - Parâmetros utilizados e resultados obtidos com algoritmo da rede recorrente Hopfield para o teste com sinal experimental real. . . . . . . p. 120

Tabela 3.15 - Parâmetros utilizados e resultados obtidos com algoritmo dos mínimos quadrados regularizado para o teste com sinal experimental real. . . . p. 122 



\section{SUMÁRIO}

$\begin{array}{lll}1 \text { Introdução } & \text { p. } 25\end{array}$

1.1 Motivação . . . . . . . . . . . . . . . . . . . . p. 25

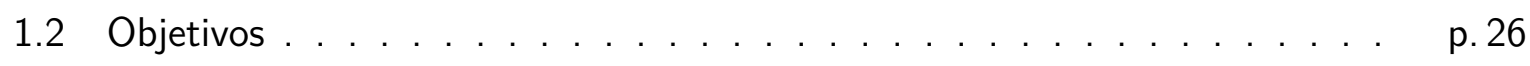

1.3 Organização do trabalho . . . . . . . . . . . . . . p. 26

2 Revisão bibliográfica $\quad$ p. 29

2.1 Ressonância magnética nuclear . . . . . . . . . . . . . . . . . . . p. 29

2.1.1 Relaxação transversal . . . . . . . . . . . . . . . . . . . p. 32

2.1.2 Relaxação longitudinal . . . . . . . . . . . . . . . . . . p. 32

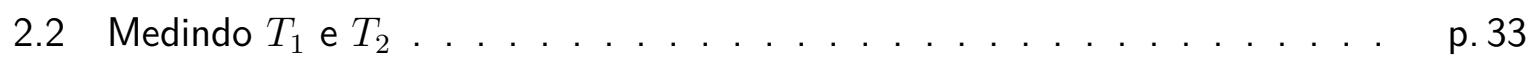

2.2.1 Free induction decay . . . . . . . . . . . . . . . . . . . . p.33

2.2 .2 Inversão-recuperação . . . . . . . . . . . . . . . . . . . . . p. 34

2.2.3 Saturação-recuperação . . . . . . . . . . . . . . . . . . . . p. 35

2.2 .4 Spin-eco e CPMG . . . . . . . . . . . . . . . . . . . . p. 35

2.3 Relaxação de líquidos em meios porosos . . . . . . . . . . . . . . . p. 37

2.4 Relaxação e tamanho dos poros . . . . . . . . . . . . . . . . . . . p. 39

2.5 Equações integrais de Fredholm e problemas inversos mal-postos . . . . . . p p. 40

2.6 Algoritmo dos mínimos quadrados médios ( LMS - Least Mean Square) . . p. p. 41

2.6 .1 Introdução . . . . . . . . . . . . . . . . . . . . . p. p. 41

2.6.2 Filtragem adaptativa - combinador linear adaptativo . . . . . . . p. p. 42

2.6.2.1 Estrutura do CLA . . . . . . . . . . . . . p. 42

2.6.2.2 Algoritmo do gradiente descendente . . . . . . . . p. 46

2.6.3 Desenvolvimento do algoritmo LMS por meio do problema da filtragem adaptativa . . . . . . . . . . . . . . . . . . . . . p. 48 
2.6.4 Obtendo distribuições $T_{2}$ com LMS . . . . . . . . . . . . p. 51

2.6.5 Algoritmo LMS com restrição de não-negatividade (LMS-NN) . . . p. p. 55

2.6.6 Algoritmo LMS regularizado e não-negativo (LMS-RNN) . . . . . . p p. 57

2.7 Redes recorrentes de Hopfield . . . . . . . . . . . . . . . . . . p p. 58

2.7.1 Funcionamento da rede de Hopfield . . . . . . . . . . . . . . . p. 61

2.7.2 Obtendo distribuições $T_{1}, T_{2}$ com as redes de Hopfield . . . . . . . p. 62

2.8 Solução dos mínimos quadrados e decomposição em valores singulares (SVD) com regularização de Tikhonov . . . . . . . . . . . . . . . p. 64

2.8 .1 SVD . . . . . . . . . . . . . . . . . . . . 64

2.8.2 Algoritmo dos mínimos quadrados (LS - Least Squares . . . . . . . p. p. 66

2.8.3 Determinação das distribuições de tempo de relaxação . . . . . . . p. p. 67

3 Técnica Proposta, Testes e Resultados p. 71

3.1 Metodologias . . . . . . . . . . . . . . . . . . p. 71

3.1 .1 LMS-NN e LMS-RNN . . . . . . . . . . . . . . . . . p. 72

3.1.2 Redes recorrentes de Hopfield . . . . . . . . . . . . . . . . . p.73

3.1.3 Solução dos mínimos quadrados com decomposição em valores singulares e regularização de Tikhonov . . . . . . . . . . . . . p.73

3.2 Teste 1 - distribuições simuladas próximas . . . . . . . . . . p. 73

3.2 .1 LMS-NN . . . . . . . . . . . . . . . . . . . . . p. 74

3.2 .2 LMS-RNN . . . . . . . . . . . . . . . . . . . . . . . . p. 79

3.2 .3 Hopfield . . . . . . . . . . . . . . . . . . . . p. 84

3.2 .4 LS-R $(S V D) \ldots \ldots \ldots \ldots$

3.3 Teste 2 - distribuições simuladas espaçadas . . . . . . . . . . . . . p. 92

3.3 .1 LMS-NN . . . . . . . . . . . . . . . . . . . . . . p. 92

3.3.2 LMS-RNN . . . . . . . . . . . . . . . . . . . . p. 97

3.3.3 Hopfield . . . . . . . . . . . . . . . . . . . . . . . . p. 102 


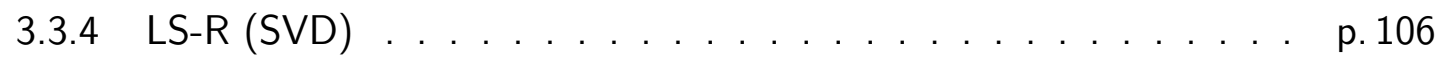

3.4 Teste 3 - distribuição delta de Kronecker . . . . . . . . . . . . . . . . . . p. 110

3.5 Teste 4 - sinal real . . . . . . . . . . . . . . . . . . . . . . p. 114

3.5 .1 LMS-NN . . . . . . . . . . . . . . . . . . . . . . . . . 114

3.5 .2 LMS-RNN . . . . . . . . . . . . . . . . . . p. 117

3.5 .3 Hopfield . . . . . . . . . . . . . . . . . . . . p. 120

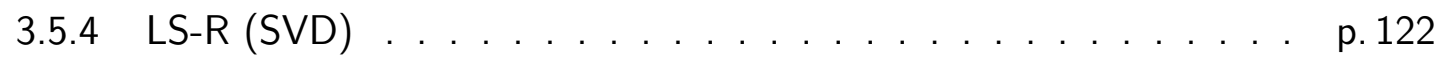

4 Conclusões e sugestões para trabalhos futuros $\quad$ p. 125

$\begin{array}{lr}\text { REFERENNCIAS } & \text { p. } 129\end{array}$

Apêndice A - Código C++ para LMS-NN e LMS-RNN p. 133

Apêndice B - Código C++ para rede recorrente de Hopfield $\quad$ p. 141

Apêndice C - Código C++ para LS-R p. 147 

CAPÍtulo 1

\section{Introdução}

Neste capítulo serão apresentadas as motivações, os principais objetivos e a organização geral do trabalho.

\subsection{Motivação}

As técnicas de processamento digital de sinais tem representado importante papel em avanços nas mais diversas áreas do conhecimento científico como, engenharia, física, biologia, economia, dentre outras. Tendo em vista a existência de uma dependência computacional intrínseca, observa-se que as possibilidades envolvendo processamento de sinais digitais, seja na análise, extração ou manipulação de informações, são influenciadas pela evolução do poder computacional, que por sua vez, a muitos anos tem apresentado forte crescimento. Além disso, a acessibilidade a esse alto poder computacional também tem crescido muito rapidamente. Neste cenário propício, a investigação de uma abordagem envolvendo processamento digital de sinais na solução de determinados problemas é bastante promissora. Esta investigação se justifica principalmente nos casos em que os métodos empregados atualmente apresentam custo computacional elevado ou mesmo resultados pouco satisfatórias, como ocorre no problema específico abordado neste trabalho.

Sob outro ponto de vista, tem-se ainda a possível extensão de impacto dos resultados, uma vez que apesar do problema em estudo, a determinação da distribuição dos tempos de relaxação $T_{1}$ e $T_{2}$ de um sinal de decaimento ou recuperação de magnetização, ser bastante específico, as abordagens são válidas para toda uma classe de problemas que surge com bastante frequência na física e engenharia, conhecida como problemas inversos mal-postos. 


\subsection{Objetivos}

O objetivo principal deste trabalho foi verificar a aplicabilidade e confrontar os resultados de alguns métodos de processamento digital de sinais na determinação de tempos de relaxação longitudinal $\left(T_{1}\right)$ e transversal $\left(T_{2}\right)$ em sinais de decaimento e recuperação de magnetização obtidos em experimentos de ressonância magnética nuclear (RMN) envolvendo meios porosos.

Particularmente, objetivou-se obter as distribuições de tempos de relaxação através de três diferentes abordagens envolvendo métodos de processamento digital de sinais. A primeira, através de filtragem adaptativa comparando duas versões do algoritmo dos mínimos quadrados médios(Least Mean Squares - LMS): LMS com restrição de não-negatividade (LMS-NN) e LMS com restrição de não-negatividade e regularização de Tikhonov (LMS-RNN), seguida da abordagem envolvendo uma arquitetura de rede neural artificial (RNA) conhecida como rede recorrente de Hopfield e, finalmente, para efeito de comparação, o método atualmente mais utilizado na solução de problemas inversos mal-postos, mínimos quadrados (Least Squares $L S)$ regularizado com decomposição em valores singulares (SVD).

\subsection{Organização do trabalho}

Tendo em vista os objetivos listados anteriormente o trabalho foi organizado em três capítulos subdivididos em seções e sub-seções, quando necessário. O capítulo 1 contém os aspectos gerais do trabalho, o capítulo 2 é constituído de breve revisão de literatura descrevendo conceitos teóricos sobre ressonância magnética nuclear, dos sinais objetos de investigação deste trabalho e sua conexão com as equações de Fredholm e os problemas inversos mal-postos. Contém também a fundamentação teórica da filtragem adaptativa e do algoritmo dos mínimos quadrados médios, seguido de duas variações do mesmo e da sua utilização na análise da distribuição dos tempos de relaxação transversal e longitudinal nos sinais de decaimento e recuperação de magnetização. Em seguida são apresentados alguns conceitos teóricos de redes neurais artificiais, a fundamentação teórica da rede recorrente de Hopfield e sua utilização na análise da distribuição dos tempos de relaxação transversal e longitudinal nos sinais de decaimento e recuperação de magnetização. Por fim, apresenta-se a decomposição em valores singulares e a solução dos mínimos quadrados regularizada e sua aplicação na solução do problema proposto. O capítulo 3 contém a descrição da metodologia empregada, das análises realizadas, os resultados obtidos e discussões referentes a estes. Finalmente no capítulo 
4 apresenta-se as conclusões, os comentários finais e as possíveis sugestões para trabalhos futuros. 


\section{Revisão bibliográfica}

Neste capítulo os conceitos necessários ao desenvolvimento do trabalho são apresentados de forma breve e em um nível de profundidade suficiente para a compreensão do estudo.

\subsection{Ressonância magnética nuclear}

O fenômeno da ressonância magnética nuclear foi descoberto independentemente por dois grupos de cientistas liderados respectivamente por Bloch (1) e Purcell.(2) A ressonância magnética é consequência da interação entre campos magnéticos externos e determinados núcleos atômicos que possuem momento magnético $\boldsymbol{\mu}$ e momento angular $\mathbf{J}$, os quais se relacionam através da seguinte expressão

$$
\boldsymbol{\mu}=\gamma \mathbf{J}
$$

onde $\gamma$ é uma propriedade intrínseca de cada núcleo atômico e conhecida como razão giromagnética.

Se imaginarmos um sistema contendo $N$ núcleos magnéticos idênticos distantes o suficiente para que as interações entre eles possam ser desprezadas e em uma configuração inicial onde todas as direções dos momentos angulares estão distribuídas aleatoriamente com todas as orientações sendo igualmente prováveis, o momento angular total e as projeções da magnetização serão nulas ao longo de qualquer direção.

Ao submeter este sistema a um campo magnético externo $\mathbf{B}_{0}$, definido na direção do eixo $z$, os momentos irão realizar um movimento de precessão em torno da direção de $\mathbf{B}_{0}$ induzido pelo torque $\boldsymbol{\mu} \times \mathbf{B}_{0}$ e o momento angular $\mathbf{J}$, conforme ilustra a figura 2.1. A equação deste movimento de precessão é obtida relacionando a taxa de variação do momento angular e o torque, isto é

$$
\frac{d \mathbf{J}}{d t}=\boldsymbol{\mu} \times \mathbf{B}_{0}
$$


Figura 2.1 - Diagrama do movimento de precessão do spin nuclear sujeito a um campo magnético externo $\mathbf{B}_{0}$.

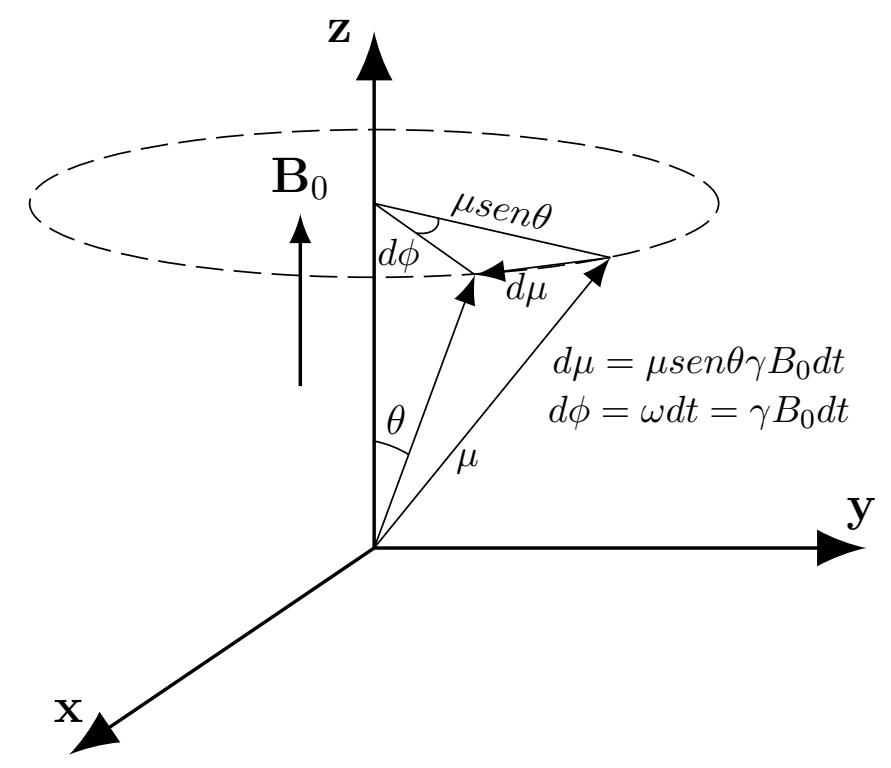

Fonte: Adaptada de D' EURYDICE. (3)

Combinando as equações (2.1.1) e (2.1.2), tem-se que

$$
\frac{d \boldsymbol{\mu}}{d t}=\boldsymbol{\mu} \times\left(\gamma \mathbf{B}_{\mathbf{0}}\right),
$$

onde a quantidade $\gamma B_{0}$, chamada de frequência de Larmor, é a frequência natural de precessão do spin submetido ao campo externo $B_{0}$. A frequência de Larmor é a frequência de ressonância do núcleo magnético, é somente nesta frequência que o núcleo magnético pode absorver ou emitir energia. A variação angular $d \phi$ devido ao movimento de precessão é então dada por

$$
d \phi=\gamma B_{0} d t
$$

A ressonância magnética é observada quando um sistema de spins com níveis de energia magnética

$$
E_{m}=-\boldsymbol{\mu} \cdot \mathbf{B}_{0}=-\gamma \hbar \mathbf{I} \cdot \mathbf{B}_{0}=-\gamma \hbar B_{0} m
$$

onde $\hbar$ é a constante de Planck, I é o operador de momento angular, separados pelo intervalo

$$
\Delta E=E_{m}-E_{m-1},
$$

chamado níveis de Zeeman, é irradiado com fótons de energia $\hbar \omega=\Delta E$. O campo magnético oscilante associado a radiação eletromagnética induz transições entre os estados de energia $E_{m}$ do sistema, o qual absorve a energia. Em equilíbrio térmico a probabilidade de encontrar 
Figura 2.2 - Diagrama de um sistema com dois níveis de energia.

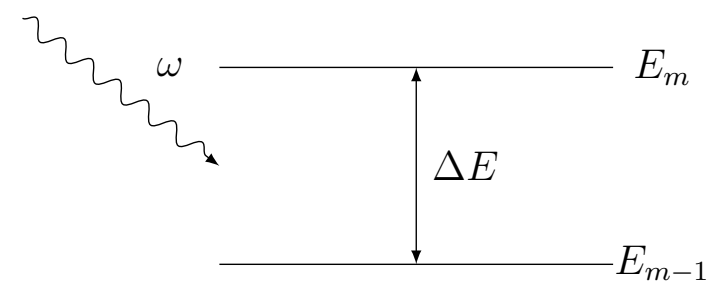

Fonte: Elaborada pelo autor.

um núcleo em um estado particular de energia $E_{m}$ é dada por (4):

$$
P_{m}=\frac{\exp \left(-E_{m} / k T\right)}{\sum_{m} \exp \left(-E_{m} / k T\right)}
$$

Deste modo, a quantidade de núcleos atômicos ocupando o nível energético $E_{m}$ pode ser definido como:

$$
N_{m}=\frac{N \exp \left(-E_{m} / k T\right)}{\sum_{m} \exp \left(-E_{m} / k T\right)}
$$

Tendo em vista que $m$ varia de $-I$ a $+I$, o somatório no denominador da equação (2.1.8) se estende também de $-I$ a $+I$, sendo $+I$ o estado menos energético, paralelo a $\mathbf{B}_{0} \mathrm{e}-I$ o estado mais energético, anti-paralelo ao campo aplicado. Tomando como exemplo o átomo de hidrogênio $(I=1 / 2)$, onde há apenas dois estados quantizados $m= \pm 1 / 2$, em equilíbrio térmico a razão das populações é dada por

$$
\frac{N_{-}}{N_{+}}=e^{-\mu B_{0} / k T}=e^{-\gamma \hbar B_{0} / k T}
$$

Nota-se que a energia magnética para um campo $B_{0}=250$ Gauss, que leva os prótons a precessionarem a uma frequência de $1 \mathrm{Mhz}$, é da ordem de $6,6 \times 10^{-26} \mathrm{~J}$, de modo que a razão das populações em temperatura ambiente é $\frac{N_{-}}{N_{+}} \approx 1-1.6 \times 10^{-5}$, ou seja, a diferença entre as populações $N_{-}$e $N_{+}$é extremamente pequena, entretanto é esta pequena diferença que leva a uma magnetização $\mathbf{M}_{0}$ observável paralela ao campo $\mathbf{B}_{\mathbf{0}}$.

A magnetização total $\mathbf{M}_{0}$ em um sistema de dois níveis pode ser definida em função da razão das populações em cada nível energético, ou seja,

$$
\mathbf{M}_{0}=N \boldsymbol{\mu}\left(1-\frac{N_{-}}{N_{+}}\right)
$$




\subsubsection{Relaxação transversal}

A relaxação transversal, como o próprio nome sugere ocorre no plano transversal e está relacionada com a perda de coerência da magnetização nesse plano. Esta perda de coerência ocorre devido à variação do campo magnético ao longo da amostra, decorrente da não homogeneidade de $B_{0}$ ou mesmo dos campos magnéticos locais produzidos pelos momentos magnéticos dos núcleos atômicos. Anteriormente foi visto que submeter o sistema de spins a um campo magnético $\mathbf{B}_{0}$ na direção do eixo $z$ faz com que uma magnetização $M_{0}$ apareça paralela a direção do campo. Na presença de um pulso de radio frequência (rf) induzido $\mathbf{B}_{1}$ é possível tirar o sistema do equilíbrio. (3) A largura do pulso de rf pode ser ajustada por meio da seguinte relação:

$$
\theta=\gamma B_{1} \Delta t
$$

Se o pulso de radio frequência $\mathbf{B}_{1}$ for ajustado para $90^{\circ}$, inicialmente os spins irão precessionar em fase no plano $x y$ (com $M_{z}$ nula) ao redor do eixo $z$ (no referencial do laboratório). Em seguida, gradualmente o movimento entre os spins perderá coerência, isto é, ocorrerá defasamento e a magnetização $M_{z}$ reaparece. O processo continua até que eventualmente $M_{z}$ retorne ao valor de equílibrio $M_{0}$.

No referencial girante, os decaimentos $M_{x}$ e $M_{y}$ são descritos pelas equações de Bloch

$$
\frac{d M_{x}}{d t}=-\frac{M_{x}}{T_{2}}, \quad \frac{d M_{y}}{d t}=-\frac{M_{y}}{T_{2}}
$$

cuja solução é também da forma exponencial, dada por:

$$
M_{\perp}(t)=M_{0} e^{-t / T_{2}}
$$

onde $T_{2}$ é o tempo de relaxação transversal, também conhecido como tempo de relaxação spin-spin.

\subsubsection{Relaxação longitudinal}

O ambiente local de um núcleo magnético cercado de moléculas é normalmente descrito como rede (termo derivado da RMN em sólidos).

Após anular a magnetização no eixo $z$ submetendo o sistema a um pulso de $\mathrm{rf}$ de $90^{\circ}$, a 
recuperação da magnetização $M_{0}$ ao longo deste eixo ocorre com um tempo característico $T_{1}$. Conforme introduzido por Bloch (1) a variação da magnetização $M_{z}$ no tempo é dada pela equação

$$
\frac{d M_{z}}{d t}=\frac{M_{0}-M_{z}}{T_{1}}
$$

cuja solução, intuitivamente, é dada na forma exponencial por

$$
M_{z}(t)=M_{0}\left(1-e^{-t / T_{1}}\right)
$$

A constante $T_{1}$, conhecida como tempo de relaxação longitudinal, é o tempo característico após o qual o sistema, se perturbado, retornará a configuração de equilíbrio térmico(5), ou seja, $T_{1}$ reflete quão efetivamente o sistema de spins troca energia magnética com o seu ambiente (rede) e por este motivo é chamada também de relaxação spin-rede.

\subsection{Medindo $T_{1}$ e $T_{2}$}

Conforme visto anteriormente, em experimentos de RMN, os fenômenos da relaxação da magnetização dos spins nucleares são descritos pelas equações de Bloch 2.1.14 e 2.1.12 e dependentes da interação entre os núcleos magnéticos e os campos locais flutuantes na amostra.

\subsubsection{Free induction decay}

A aquisição do sinal induzido após um pulso de $90^{\circ}$, conhecido como Free Induction Decay (FID), é o experimento mais simples realizado em RMN. A magnitude do sinal no FID, após

Figura 2.3 - Free Induction Decay.

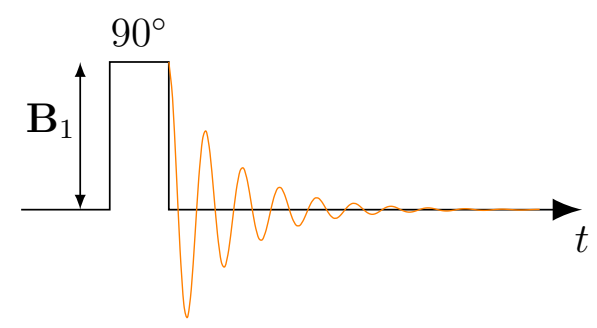

Fonte: Elaborada pelo autor. 
cessar o pulso de radio frequência, decai exponencialmente com uma constante de tempo $T_{2}^{*}$, a qual está relacionada com o tempo de relaxação transversal através da equação

$$
\frac{1}{T_{2}^{*}}=\frac{1}{T_{2}}+\frac{\gamma \Delta B_{0}}{2}
$$

onde $\Delta B_{0}$ é o desvio de $B_{0}$ devido a inomogeneidade do campo.

No FID, além das interações dos núcleos com os campos locais, a perda de coerência no plano transversal está relacionada principalmente com a inomogeneidade do campo $\mathbf{B}_{0}$, que acelera o defasamento entre os spins. Nota-se, portanto, que o tempo de relaxação no FID não depende apenas das propriedades intrínsecas da amostra o que o torna ineficiente para a determinação de $T_{2}$.

\subsubsection{Inversão-recuperação}

A sequência inversão-recuperação (IR) é utilizada na medida de $T_{1}$ e tem como vantagem eliminar quase totalmente a influência de $T_{2}$, tendo em vista que o vetor magnetização é totalmente longitudinal. A sequência consiste de um pulso de $180^{\circ}$, que inverte a magnetização no eixo $z$, seguido de um tempo de recuperação $\tau$ variável e um pulso de leitura de $90^{\circ}$. (6)

Figura 2.4 - Sequência de inversão-recuperação, utilizada na medida de $T_{1}$.

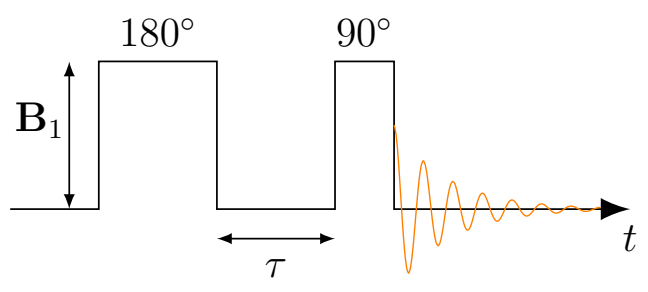

Fonte: Elaborada pelo autor.

O comportamento da magnetização longitudinal na sequência inversão-recuperação é dado pela equação 2.2.2, entretanto deve-se observar que este comportamento não pode ser medido diretamente, tendo em vista que não há componente transversal (7) de modo que $M_{z}$ é obtida indiretamente, repetindo-se a sequência da figura 2.4 com diferentes valores de $\tau$ e realizando um ajuste de curva.

$$
M_{z}(\tau)=M_{0}\left(1-2 e^{-\tau / T_{1}}\right) .
$$




\subsubsection{Saturação-recuperação}

Na sequencia saturação-recuperação $(\mathrm{SR})$, também utilizada na medida de $T_{1}$, a magnetização é transferida para o plano transversal $\left(M_{z}=0\right)$ através de um pulso de $90^{\circ}$ e permite-se que ocorra defasamento durante um período $\tau_{1}$, este processo é repetido diversas vezes até que ocorra efetiva saturação na magnetização (8) tornando-a nula na direção $z$. Com o sistema saturado, após um período $\tau_{2}$, aplica-se um pulso de leitura $\left(90^{\circ}\right)$ colocando novamente a magnetização no plano transversal.

Figura 2.5 - Sequência de saturação-recuperação, utilizada na medida de $T_{1}$.

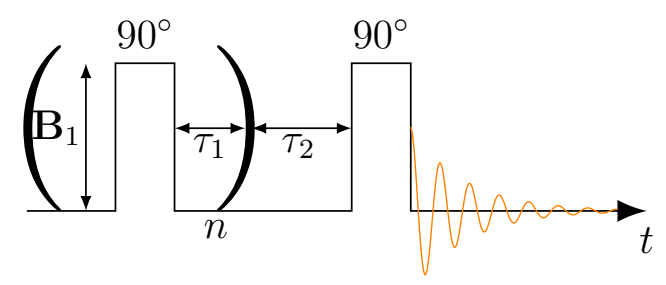

Fonte: Elaborada pelo autor.

O procedimento é repedido diversas vezes variando o tempo de recuperação $\tau_{2}$. A expressão que descreve a evolução da magnetização neste experimento é dada pela equação seguinte

$$
M_{z}\left(\tau_{2}\right)=M_{0}\left(1-e^{-\tau_{2} / T_{1}}\right)
$$

\subsubsection{Spin-eco e CPMG}

Conforme visto anteriormente o FID não é adequado para a medida de $T_{2}$, tendo em vista que além dos efeitos das flutuações de campos locais na amostra o decaimento é acelerado pela inomogeneidade do campo $B_{0}$. Em um trabalho publicado por Erwin Hahn em 1950 (9) surgiu a proposta de um novo experimento que permitiria medir o tempo de relaxação transversal evitando os efeitos do campo magnético não homogêneo, a proposta de Hahn, que ficou conhecida como spin-eco, consistia de um pulso de $90^{\circ}$ seguido de outro pulso de $90^{\circ}$ separados por um intervalo $\tau<T_{2}$. Baseado na proposta de Hahn, os pesquisadores Carr e Purcell, em 1954 (10), propuseram substituir o segundo pulso de $90^{\circ}$ por um pulso de $180^{\circ}$ separados por um intervalo $2 \tau$ visando obter uma maior intensidade de eco que ocorre exatamente no centro destes intervalos. 
Figura 2.6 - Refocalização com pulso de $180^{\circ}$, utilizado na medida de $T_{2}$.

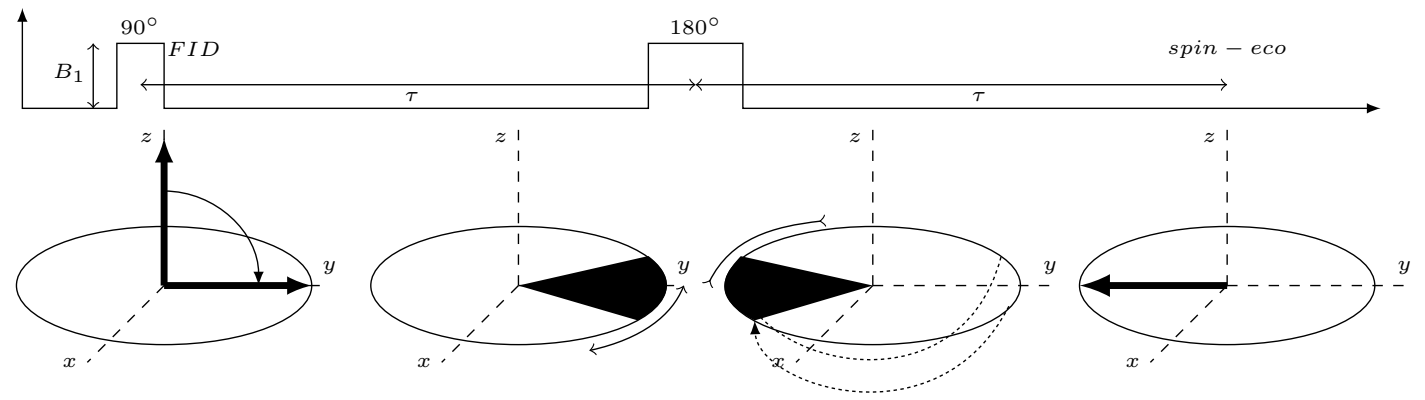

Fonte: Elaborada pelo autor.

Conforme ilustrado na figura 2.6, logo após o pulso de $90^{\circ}$ os spins começam a perder a coerência devido a não-homogeneidade de $B_{0}$ e à interação com os campos dipolares locais. Após o sistema relaxar por um período de tempo $\tau$ aplica-se um pulso de $180^{\circ} \mathrm{com}$ o objetivo de refocalizar os spins, isto é, o pulso gira os spins de um ângulo de $180^{\circ}$ no plano transversal, mas mantém suas velocidades de modo que eles voltam a entrar em fase resultando em um novo aumento no sinal medido. A re-focalização ocorre no mesmo período de tempo $\tau$ e elimina os efeitos da não-homogeneidade de $B_{0}$. Entretanto, os efeitos dos campos dipolares locais são mantidos, de modo que, conforme ilustrado na figura 2.7, o sinal de magnetização alcança uma intensidade máxima menor que a intensidade medida logo após cessar o pulso de $90^{\circ}$.

Figura 2.7 - Fenômeno spin-eco, utilizado na medida de $T_{2}$.

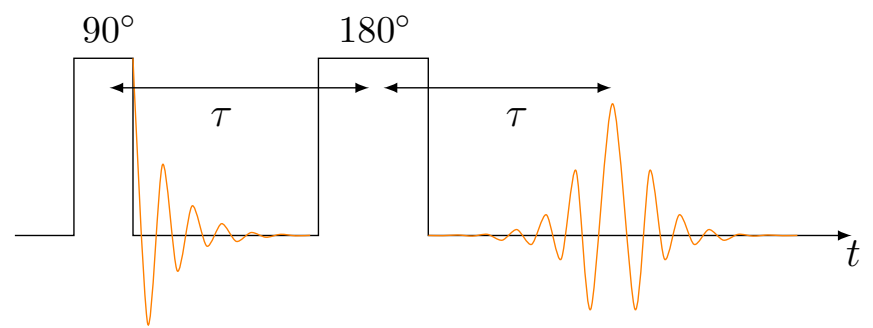

Fonte: Elaborada pelo autor.

A sequência proposta por Carl e Purcell passou a ser conhecida como CPMG após a contribuição de S. Meiboom e D. Gill (11), os quais introduziram a ideia de que os pulsos sucessivos devem ser coerentes e que a fase do pulso de rf de $90^{\circ}$ deve ser deslocada em $90^{\circ}$ de modo a eliminar as imperfeições dos pulsos de $180^{\circ}$. 
Figura 2.8 - Sequência de pulsos na medida de $T_{2}$ proposta por Carl, Purcell, Meiboom e Gill.

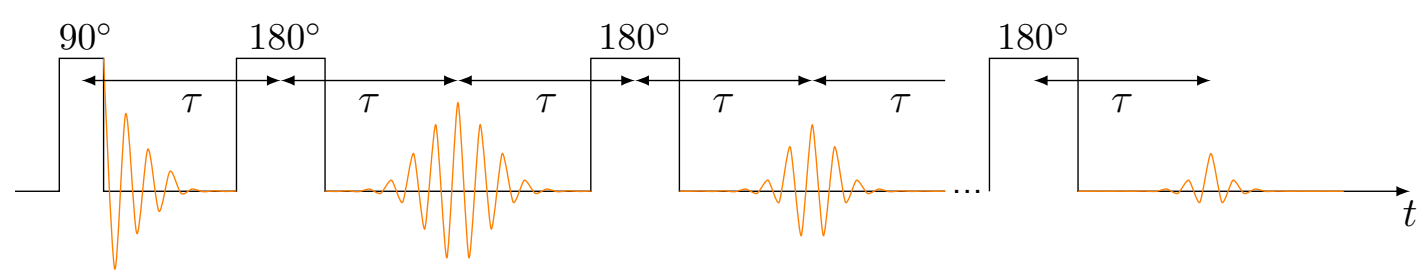

Fonte: Elaborada pelo autor.

\subsection{Relaxação de líquidos em meios porosos}

A relaxação dos líquidos em meios porosos envolve três mecanismos principais independentes (12), processo de relaxação bulk, relaxação de superfície e difusão na presença de gradientes de campo magnético, os quais estão associados a dinâmica molecular dos líquidos. Durante um experimento de RMN as moléculas percorrem distâncias relativamente grandes, eventualmente migram para outros poros e interagem com a superfície destes modificando as propriedades de relaxação. Os processos de relaxação atuam em paralelo em $T_{1}$ e $T_{2}$, porém atuam de maneiras distintas.

O tempo de relaxação $T_{1}$ é resultado da soma dos inversos das contribuições que o afeta, isto é, a relaxação bulk, que é a relaxação intrínseca do fluído, dependente de suas propriedades físico-químicas e a relaxação de superfície que ocorre na interface fluído-sólido dos poros.

$$
\frac{1}{T_{1}}=\frac{1}{T_{1_{b}}}+\frac{1}{T_{1_{\mathrm{s}}}}
$$

Em $T_{2}$ além das contribuições da relaxação bulk e da relaxação de superfície há também a contribuição da difusão devido a interação com os gradientes de campo magnético

$$
\frac{1}{T_{2}}=\frac{1}{T_{2_{b}}}+\frac{1}{T_{2_{\mathrm{s}}}}+\frac{1}{T_{2_{\mathrm{d}}}} .
$$

Muitas vezes, os processos de relaxacão bulk de líquidos e gases confinados em meios porosos são desprezíveis (13), $T_{b} \gg T_{s}$, de modo que a relaxação é dominada pelos processos de relaxação de superfície e difusão do fluído. Em análises teóricas realizadas por Brownstein e Tarr (14) foram identificados três regimes distintos de relaxação fluído-sólido, os quais são determinados pela relação $T_{s} / D$, onde $T_{s}$ é determinado, primariamente, pela relaxatividade 
Figura 2.9 - Possíveis mecanimos de relaxação no interior de um poro.

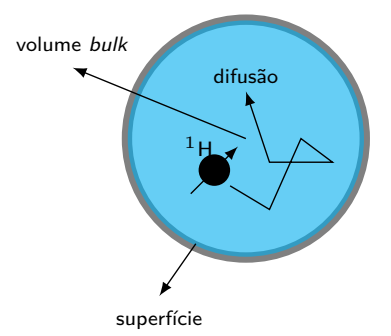

Fonte: Elaborada pelo autor.

superficial $(\rho)$ e pela geometria do poro, isto é,

$$
\frac{1}{T_{s}}=\rho_{1,2} \frac{S}{V} \equiv \rho_{1,2} r_{p}
$$

regime de difusão rápida $\rho_{1,2} r_{p} / D \ll 1$, ou seja, o decaimento da magnetização macroscópica é dominado pela relaxação dos núcleos com a superfície, portanto,

$$
\begin{gathered}
\frac{1}{T_{1_{s}}}=\rho_{1} \frac{S}{V}, \\
\frac{1}{T_{2_{s}}}=\rho_{2} \frac{S}{V} .
\end{gathered}
$$

No regime de difusão rápida, a relaxação é uniforme e apresenta comportamento exponencial (15), mais especificamente, a relaxação de um único poro é comandada por uma função mono-exponencial dependente da relação superfície/volume do poro.

regime de difusão intermediária $\rho_{1,2} r_{p} / D \approx 1$, apesar do decaimento da magnetização macroscópica ainda ser dominado pelo processo de relaxação de superfície, neste caso os dois processos contribuem com o decaimento da magnetização, que apresenta comportamento multi-exponencial.

regime de difusão lenta $\rho_{1,2} r_{p} / D \gg 1$, em geral os núcleos relaxam rapidamente em contato com a superfície e a difusão é lenta. O decaimento da magnetização macroscópica apresenta comportamento multi-exponencial, mesmo para um único poro.

Neste trabalho serão considerados apenas fluídos em regime de difusão rápida contidos em meios porosos, cujos poros são desconectados e nos quais o regime de relaxação é limitado a superfície, de modo que, conforme visto anteriormente, o decaimento da magnetização de cada poro é mono-exponencial e dependente apenas da razão superfície/volume. 


\subsection{Relaxação e tamanho dos poros}

Em geral, um material poroso possui uma distribuição ampla de tamanho de poros nos quais o tempo de relaxação, conforme ilustrado na figura 2.10, é diretamente proporcional ao tamanho do poro, portanto o decaimento da magnetização é na verdade uma soma de exponenciais com diferentes tempos de relaxação. Consequentemente a evolução da magneti-

Figura 2.10 - Representação da relação entre tempos de relaxação e tamanho do poro.

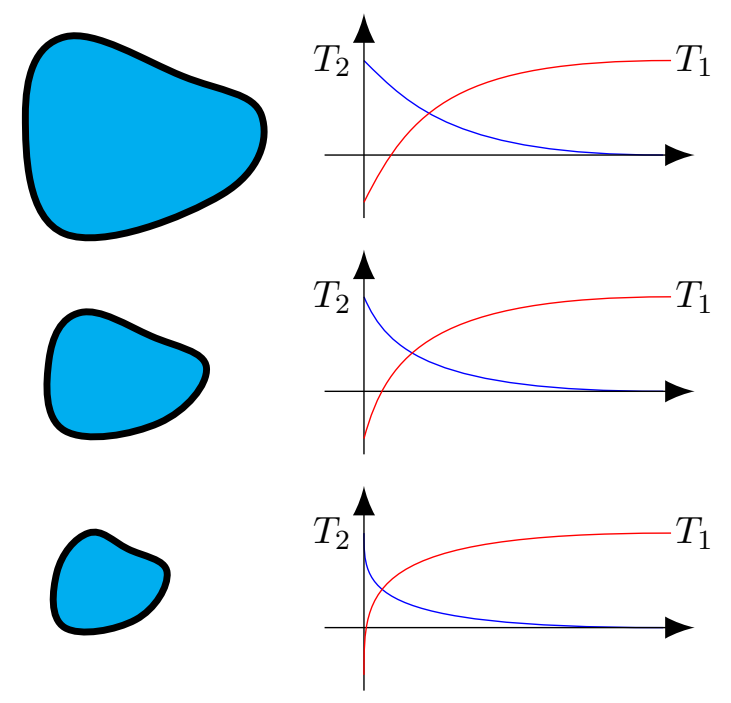

Fonte: Elaborada pelo autor.

zação nos experimentos inversão-recuperação, saturação-recuperação e CPMG são descritos, de forma geral, através das seguintes equações integrais:

\section{Inversão-Recuperação}

$$
M_{\|}(t)=\int_{T_{1_{\min }}}^{T_{1_{\max }}} g\left(T_{1}\right)\left(1-2 e^{-\tau / T_{1}}\right) d T_{1}
$$

\section{Saturação-Recuperação}

$$
M_{\|}(t)=\int_{T_{1_{\min }}}^{T_{1_{\max }}} g\left(T_{1}\right)\left(1-e^{-\tau / T_{1}}\right) d T_{1}
$$

\section{CPMG}

$$
M_{\perp}(t)=\int_{T_{2_{\min }}^{T_{2 \max }}}^{T_{2}} g\left(T_{2}\right) e^{-\tau / T_{2}} d T_{2}
$$

onde $g\left(T_{1}\right)$ e $g\left(T_{2}\right)$ são, respectivamente, as distribuições de tempos de relaxação longitudinal e transversal. As equações 2.4.1, 2.4 .2 e 2.4 .3 podem ser representadas de uma forma ainda mais geral através de uma equação integral de Fredholm de primeira espécie não-homogênea. 


\subsection{Equações integrais de Fredholm e problemas inversos mal-postos}

Uma equação integral de Fredholm de primeira espécie é uma equação integral que possui a seguinte forma geral,

$$
f(x)=\int_{a}^{b} \phi(t) K(x, t) d t
$$

onde os limites de integração são constantes (em contraste com as equações integrais de Volterra, nas quais os limites são variáveis), as funções $K(x, t)$, conhecida como núcleo e $f(x)$ são conhecidas e $\phi(t)$ é uma função a ser determinada (16). Em muitas das aplicações práticas envolvendo equações integrais de Fredholm o núcleo é dado por um modelo matemático exato, enquanto $f(x)$ é tipicamente um sinal discretizado, ou seja, um conjunto finito de pontos de precisão limitada e sujeito a erros de medida, o que torna as equações integrais de Fredholm o exemplo clássico de problema inverso* mal-posto.

O conceito de problema bem-posto ou mal-posto remete ao trabalho de Jaques Hardamard (17) no fim do século passado. Essencialmente Hardamard define um problema como malposto se a solução do problema não é única ou se não é uma função contínua dos dados. Formalmente pode-se definir os seguintes critérios:

Sejam $\mathcal{X}$ e $\mathcal{Y}$ espaços normados,

existência: para cada vetor de entrada $\mathbf{x} \in \mathcal{X}$, existe uma saída $y=f(\mathbf{x})$ onde $y \in \mathcal{Y}$.

unicidade: para cada par de vetores de entrada $\mathbf{x}, \mathbf{t} \in \mathcal{X}, f(\mathbf{x})=f(\mathbf{t})$ se, e somente se $\mathbf{x}=\mathbf{t}$.

continuidade: para qualuer $\varepsilon>0$ existe $\delta=\delta(\varepsilon)$ tal que a distância em seus respectivos espaços $\rho_{x}(\mathbf{x}, \mathbf{t})<\delta$ implica em $\rho_{y}(f(\mathbf{x}), f(\mathbf{t}))<\varepsilon$.

A violação de qualquer dos critérios acima implica em um problema mal-posto.

As dificuldades envolvendo a equação integral 2.5.1 estão ainda intimamente ligadas a compacidade do operador associado ao núcleo $K(x, t)(18)$, fisicamente, a integração envolvendo o núcleo $K(x, t)$ tem efeito suavizante em $\phi(t)$, de modo que o processo inverso, isto é, determinar $f(x)$ por meio de $\phi(t)$ tende a amplificar qualquer componente de alta-frequência em $f(x)$.

\footnotetext{
* Define-se problema inverso como o oposto ao trivial e bem conhecido problema direto, o qual se resume a obter resposta de um determinado modelo a um conjunto de dados de entrada, ou seja, no problema inverso o objetivo é determinar os dados de entrada a partir dos efeitos observados.
} 
Em todas as abordagens exploradas neste trabalho serão consideradas, sem perda de generalidade, sinais de magnetização transversal, simulados ou não, obtidos por meio de sequência CPMG.

\subsection{Algoritmo dos mínimos quadrados médios (LMS - Least Mean Square)}

\subsubsection{Introdução}

O algoritmo dos mínimos quadrados médios surgiu na década de sessenta em um trabaIho sobre reconhecimento de padrões realizado por Widrow e Hoff e desde então tornou-se o algoritmo de filtragem adaptativa mais utilizado na prática. De fato, o algoritmo LMS é o carro-chefe e provavelmente grande responsável pelo desenvolvimento da filtragem adaptativa linear. Diversas adaptações do algoritmo resultaram em aplicações nas mais diversas áreas, como por exemplo, sistemas de controle, processamento digital de sinais de áudio e voz, engenharia biomédica, sismologia e etc, além de estar intimamente relacionado com o surgimento e o desenvolvimento das redes neurais artificiais (RNA). O LMS é um algoritmo de gradiente estocástico o que o diferencia do algoritmo do gradiente descendente. O sucesso do algoritmo LMS é reflexo de sua implementação intuitiva e extremamente simples frente a sua efetividade e robustez no que diz respeito a aplicação.

A proposta deste capítulo é discutir, em princípio, o comportamento, as características, a implementação e demais aspectos teóricos do chamado Combinador Linear Adaptativo (CLA), considerado a essência dos algoritmos de filtragem adaptativa, seguido da apresentação do algoritmo dos mínimos quadrados médios sob a visão de um problema de filtragem adaptativa e das abordagens de imposição de suavidade e restrição de não-negatividade nos elementos ajustáveis, visando adaptar a estrutura do algoritmo para obter solução para as equações de Fredholm ou, mais especificamente, para realizar a análise das distribuições de tempo de relaxação em sinais de decaimento proveniente dos experimentos de RMN envolvendo meios porosos. 


\subsubsection{Filtragem adaptativa - combinador linear adaptativo}

O combinador linear adaptativo é fundamental no processamento adaptativo de sinais digitais. O CLA está, de alguma forma, relacionado com a maioria dos processos adaptativos e devido a sua estrutura não recursiva, na maioria dos casos, é relativamente fácil de compreender e analisar.

\subsubsection{Estrutura do CLA}

Figura 2.11 - Forma geral do combinador linear adaptativo de múltiplas entradas

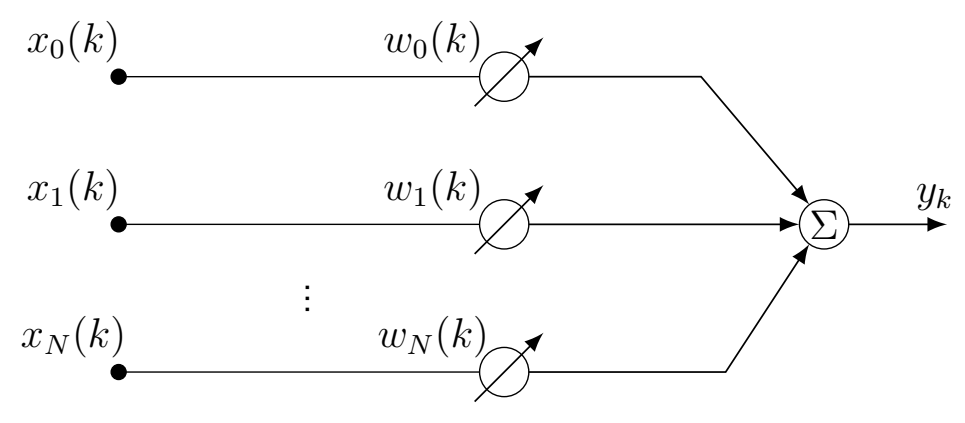

Fonte: Elaborada pelo autor.

A figura 2.11 apresenta o diagrama geral do combinador linear adaptativo. Os elementos $x_{0}, x_{1}, \ldots, x_{N}$ correspondem a um conjunto de valores de entrada conectados aos respectivos pesos ajustáveis $w_{0}, w_{1}, \ldots, w_{N}$ os quais, por sua vez, estão totalmente conectados a uma única unidade de soma compondo um sinal de saída $y_{k}$.

Os valores de entrada podem ser interpretados de duas maneiras distintas, podem ser considerados simultâneos, isto é, quando provenientes de $N+1$ diferentes fontes de sinal (múltiplas entradas) ou sequenciais quando o sinal de entrada é formado por $N+1$ amostras sequenciais da uma mesma fonte de sinal (entrada única). Tendo em vista as características do problema proposto, considera-se adiante apenas o CLA de entradas múltiplas.

Os valores de entrada e os elementos ajustáveis relacionados podem ser respectivamente representados em forma vetorial por:

$$
\begin{aligned}
\mathbf{x}_{\mathbf{k}} & =\left[x_{0}(k) x_{1}(k) \ldots x_{N}(k)\right]^{T} \\
\mathbf{w}_{\mathbf{k}} & =\left[w_{0}(k) w_{1}(k) \ldots w_{N}(k)\right]^{T}
\end{aligned}
$$


e deste modo o sinal de saída $y_{k}$ é dado por:

$$
y_{k}=\sum_{i=0}^{N} x_{i}(k) w_{i}(k)
$$

ou ainda, na forma vetorial,

$$
y_{k}=\mathbf{x}_{\mathbf{k}}^{T} \mathbf{w}_{\mathbf{k}}=\mathbf{w}_{\mathbf{k}}^{T} \mathbf{x}_{\mathbf{k}}
$$

$\mathrm{O}$ ajuste do vetor de pesos $\mathbf{w}_{\mathbf{k}}$ em malha fechada depende ainda de um sinal de resposta desejada $d_{k}$. O processo de adaptação dos parâmetros livres do CLA tende a aproximar $y_{k}$ da resposta desejada $d_{k}$ por meio da minimização do sinal de erro obtido através da comparação de $y_{k}$ e $d_{k}$, isto é,

$$
e_{k}=d_{k}-y_{k}
$$

substituindo 2.6.4 em 2.6.5 tem-se,

Figura 2.12 - Diagrama do processo adaptativo do combinador linear envolvendo o sinal de saída $y_{k}$ e o sinal desejado $d_{k}$.

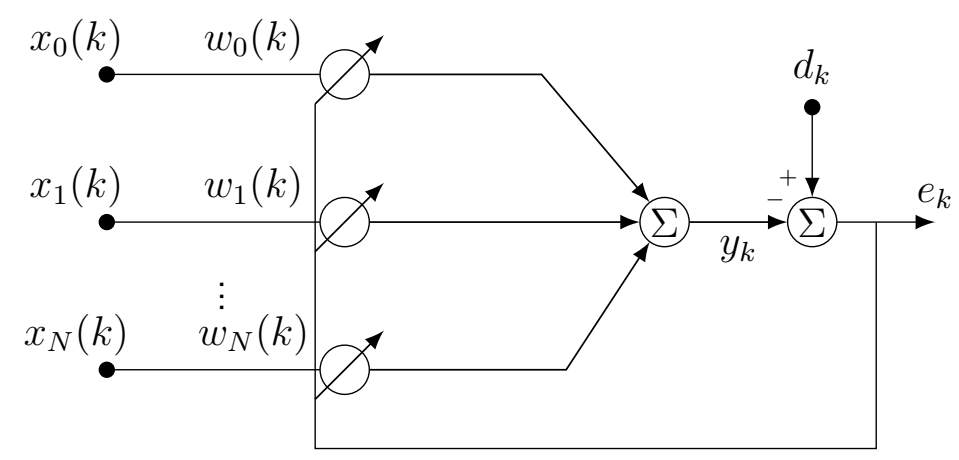

Fonte: Elaborada pelo autor.

$$
e_{k}=d_{k}-\mathbf{x}_{\mathbf{k}}^{T} \mathbf{w}_{\mathbf{k}}
$$

A equação de erro instantâneo 2.6.6 pode exibir uma estrutura complexa constituída de muitos mínimos locais, de modo que a avaliação da proximidade entre $y_{k}$ e $d_{k}$, bem como o ajuste do vetor de pesos $\mathbf{w}_{\mathbf{k}}$ é realizado considerando o valor esperado da energia do erro instantâneo, isto é, o erro médio quadrático (MSE - Mean Squared Error), dado por:

$$
\begin{gathered}
e_{k}^{2}=d_{k}^{2}+\mathbf{w}_{k}^{T} \mathbf{x}_{k} \mathbf{x}_{k}^{T} \mathbf{w}_{k}-2 d_{k} \mathbf{x}_{k}^{T} \mathbf{w}_{\mathbf{k}} \\
E\left[e_{k}^{2}\right]=\xi_{k}=E\left[d_{k}^{2}\right]+\mathbf{w}_{k}^{T} \mathbf{R} \mathbf{w}_{k}-2 \mathbf{p}^{T} \mathbf{w}_{\mathbf{k}}
\end{gathered}
$$


onde $\mathbf{R}$ é a matriz de correlação do sinal de entrada e definida como

$$
\mathbf{R}=E\left[\mathbf{x}_{k} \mathbf{x}_{k}^{T}\right]=E\left[\begin{array}{ccccc}
\mathbf{x}_{0 k}^{2} & \mathbf{x}_{0 k} \mathbf{x}_{1 k} & \mathbf{x}_{0 k} \mathbf{x}_{2 k} & \cdots & \mathbf{x}_{0 k} \mathbf{x}_{N k} \\
\mathbf{x}_{1 k} \mathbf{x}_{0 k} & \mathbf{x}_{2 k}^{2} & \mathbf{x}_{1 k} \mathbf{x}_{2 k} & \cdots & \mathbf{x}_{1 k} \mathbf{x}_{N k} \\
\vdots & \vdots & \vdots & \ddots & \vdots \\
\mathbf{x}_{N k} \mathbf{x}_{0 k} & \mathbf{x}_{N k} \mathbf{x}_{1 k} & \mathbf{x}_{N k} \mathbf{x}_{2 k} & \cdots & \mathbf{x}_{N k}^{2}
\end{array}\right]
$$

Nota-se que a diagonal principal de $\mathbf{R}$ é formada pelos valores médios quadráticos do sinal de entrada $\mathbf{x}_{k}$. De maneira similar define-se o vetor de correlação cruzada $\mathbf{p}$ entre a resposta desejada $d_{k}$ e o sinal de entrada $\mathbf{x}_{k}$ como:

$$
\mathbf{p}=E\left[d_{k} \mathbf{x}_{k}^{T}\right]=E\left[\begin{array}{llll}
d_{k} \mathbf{x}_{0 k} & d_{k} \mathbf{x}_{1 k} & \cdots & \left.d_{k} \mathbf{x}_{N k} \cdot\right]^{T}
\end{array}\right.
$$

Fica claro na expressão 2.6 .8 que o erro médio quadrático $\xi$ é precisamente uma função quadrática do vetor de pesos w quando o sinal de entrada e a resposta desejada são variáveis estocásticas estacionárias (19). A superfície definida por $\xi$ para $\mathbf{w} \in \Re^{2}$ é um paraboloide (ou hiper paraboloide para $\mathbf{w} \in \Re^{N}, N>2$ ) e é conhecida como superfície de desempenho. Conforme verifica-se na figura 2.13 a superfície de desempenho possui apenas um mínimo global o qual corresponde exatamente ao vetor de pesos ótimo $\mathrm{w}^{0}$ e que pode ser obtido diretamente por meio da seguinte equação:

$$
\frac{\partial \xi}{\partial \mathbf{w}}=\mathbf{0}
$$

onde $\frac{\partial \xi}{\partial \mathbf{w}}$ é o gradiente do erro médio quadrático $\xi$ em relação ao vetor de pesos $\mathbf{w}$, definido

Figura 2.13 - Superfície de desempenho quadrática bi-dimensional.

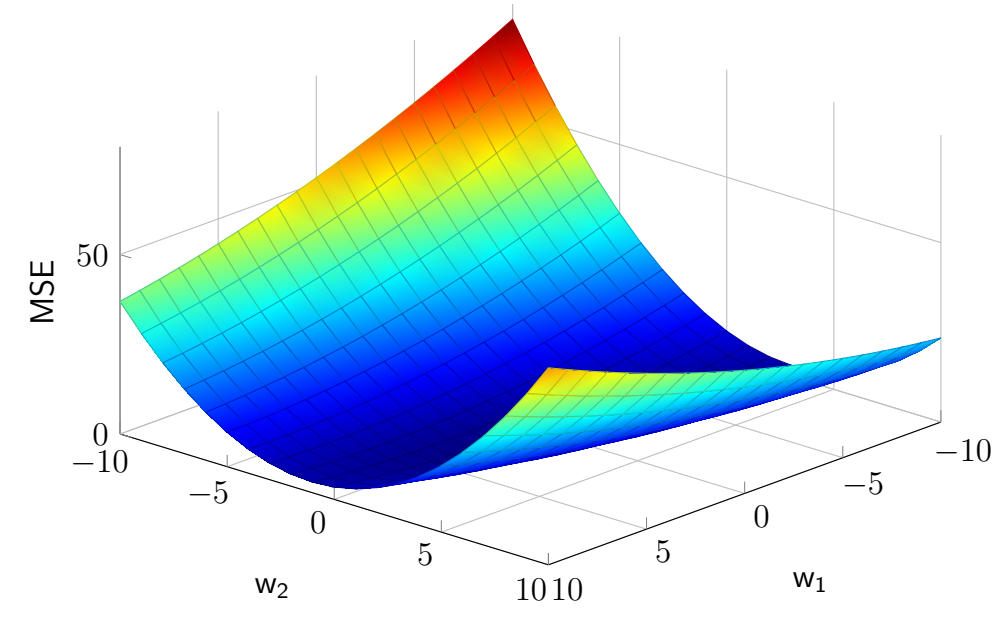

Fonte: Elaborada pelo autor. 
como

$$
\frac{\partial \xi}{\partial \mathbf{w}}=\left[\begin{array}{llll}
\frac{\partial \xi}{\partial w_{0}} & \frac{\partial \xi}{\partial w_{1}} & \cdots & \frac{\partial \xi}{\partial w_{N}}
\end{array}\right]
$$

resolvendo 2.6 .12

$$
\frac{\partial \xi}{\partial \mathbf{w}}=2(\mathbf{R w}-\mathbf{p})
$$

O valor ótimo $\mathrm{w}^{0}$ (no sentido dos mínimos quadrados médio) para os elementos ajustáveis do combinador linear é obtido igualando a expressão do gradiente 2.6.12 à zero, isto é,

$$
\frac{\partial \xi}{\partial \mathbf{w}}=\mathbf{0}
$$

portanto,

$$
\begin{gathered}
\mathbf{R w}-\mathbf{p}=\mathbf{0} \\
\mathbf{w}^{0}=\mathbf{R}^{-1} \mathbf{p}
\end{gathered}
$$

Figura 2.14 - Curvas de contorno da superfície de desempenho com a localização de $\mathbf{w}^{0}$.

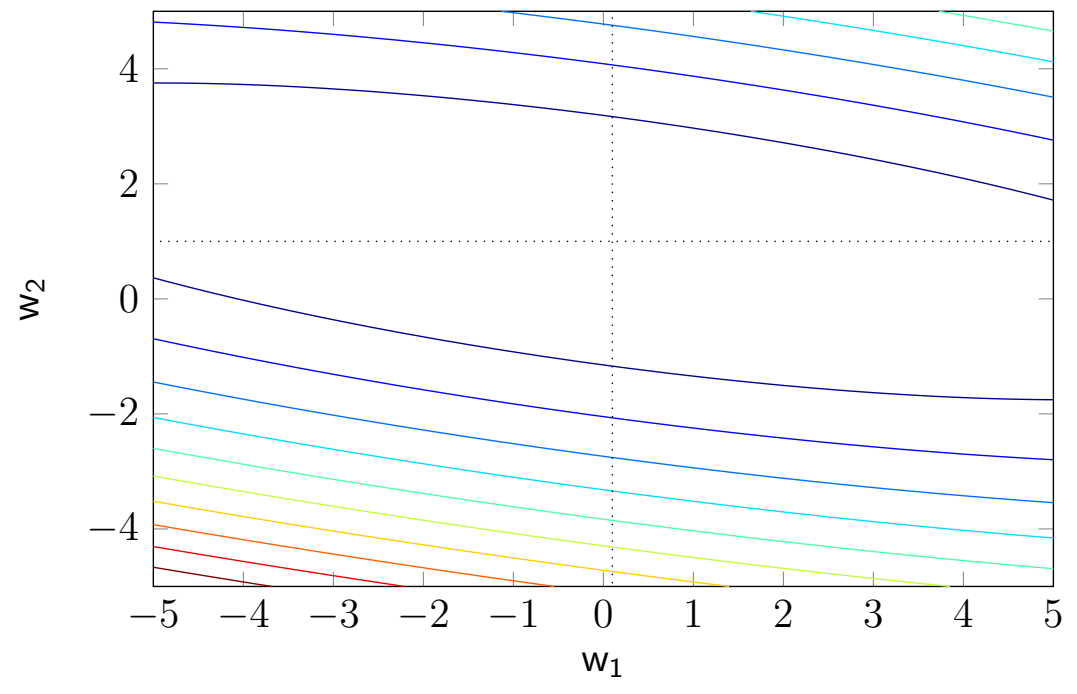

Fonte: Elaborada pelo autor.

A equação 2.6.15 é a forma matricial da expressão conhecida como solução de WienerHopf, ou simplesmente solução de Wiener. Substituindo a solução de Wiener na expressão do erro médio quadrático definida em 2.6.8, obtém-se o valor mínimo do erro médio quadrático,

$$
\begin{aligned}
\xi_{\min } & =E\left[d_{k}^{2}\right]+\mathbf{w}^{\mathbf{0}^{T}} \mathbf{R} \mathbf{w}^{\mathbf{0}}-2 \mathbf{p}^{T} \mathbf{w}^{\mathbf{0}} \\
& =E\left[d_{k}^{2}\right]+\left[\mathbf{R}^{-1} \mathbf{p}\right]^{T} \mathbf{R} \mathbf{R}^{-1} \mathbf{p}-2 \mathbf{p}^{T} \mathbf{R}^{-1} \mathbf{p}
\end{aligned}
$$

o qual, considerando a propriedade de simetria da matriz de correlação $\mathbf{R}$, pode ser reescrito 
na forma

$$
\xi_{\min }=E\left[d_{k}^{2}\right]-\mathbf{p}^{T} \mathbf{w}^{\mathbf{0}}
$$

Contudo, deve-se observar que a solução de Wiener obtida em 2.6.15 não tem muita utilidade prática tendo em vista que normalmente, em aplicações reais, os parâmetros que definem a superfície de desempenho quadrática, isto é, a matriz de correlação do sinal de entrada $\mathbf{R}$ e a matriz de correlação cruzada entre o sinal de entrada e a resposta desejada $\mathbf{p}$ não são totalmente conhecidos. Uma das possíveis abordagens para contornar o problema é utilizar um modelo iterativo de avaliação estimada da superfície de desempenho. A busca por uma solução aproximada de $\mathbf{w}^{0}$ através de um modelo iterativo traz a vantagem de permitir incluir restrições na formulação do problema visando garantir soluções coerentes levando em consideração os aspectos de má-formulação do problema inverso exposto nas sessões 2.4 e 2.5 .

\subsubsection{Algoritmo do gradiente descendente}

Considerando o modelo da figura 2.12 e assumindo que $\mathbf{R}$ e $\mathbf{p}$ são conhecidos, diferente da solução direta de Wiener-Hopf, o algoritmo do gradiente descendente tende a alcançar o vetor de pesos ótimo $\mathrm{w}^{0}$ por meio de um processo iterativo de busca, isto é, através de passos consecutivos, partindo de um valor inicial $\mathbf{w}(0)$ e avaliando o gradiente de uma função objeto convexa $\xi$ em função dos parâmetros w que a definem. (20) A cada passo do algoritimo o vetor de pesos é ligeiramente modificado na direção oposta ao gradiente até que um valor crítico de convergência seja atingido. De maneira geral o algoritmo do gradiente descendente é definido pela seguinte expressão:

$$
\Delta \mathbf{w}=-\eta \frac{\partial \xi}{\partial \mathbf{w}}
$$

onde o termo $\Delta \mathbf{w}$ à esquerda de 2.6.18 representa a diferença entre os vetores de parâmetros do CLA em duas iterações consecutivas, ou seja,

$$
\mathbf{w}(k+1)-\mathbf{w}(k)=-\eta \frac{\partial \xi}{\partial \mathbf{w}}
$$

que é, portanto, a equação de atualização dos pesos do combinador.

O parâmetro $\eta$ em 2.6.19 é conhecido como parâmetro da taxa de aprendizagem e resumidamente exprime o quão rápido o processo de treinamento do algoritmo segue em direção ao ponto de minimização de $\xi$. Os valores adotados para $\eta$ pertencem ao intervalo compreendido em $0<\eta<1(21)$. Através da análise de convergência do algoritmo é possível demonstrar 
que a escolha de um $\eta$ adequado é fundamental e que está intrinsecamente relacionada à convergência do algoritmo do gradiente descendente.

Chega-se a prova de convergência de 2.6.18 rescrevendo $\xi$, obtido em 2.6.8, da seguinte forma:

$$
\begin{aligned}
\xi_{k} & =E\left[d_{k}^{2}\right]+\mathbf{w}_{k}^{T} \mathbf{R} \mathbf{w}_{k}-2 \mathbf{p}^{T} \mathbf{w}_{\mathbf{k}} \\
& =E\left[d_{k}^{2}\right]-\mathbf{p}^{T} \mathbf{R}^{-1} \mathbf{p}+\mathbf{p}^{T} \mathbf{R}^{-1} \mathbf{p}+\mathbf{w}^{T} \mathbf{R} \mathbf{w}-2 \mathbf{w}^{T} \mathbf{p} \\
& =\xi_{\min }+\mathbf{p}^{T} \mathbf{R}^{-1} \mathbf{R} \mathbf{R}^{-1} \mathbf{p}+\mathbf{w}^{T} \mathbf{R} \mathbf{w}-2 \mathbf{w}^{T} \mathbf{p} \\
& =\xi_{\min }+\mathbf{w}^{\mathbf{0}^{T}} \mathbf{R} \mathbf{w}^{\mathbf{0}}+\mathbf{w}^{T} \mathbf{R} \mathbf{w}+2 \mathbf{w}^{T} \mathbf{R} \mathbf{w}^{\mathbf{0}} \\
& =\xi_{\min }+\left(\mathbf{w}-\mathbf{w}^{\mathbf{0}}\right)^{T} \mathbf{R}\left(\mathbf{w}-\mathbf{w}^{\mathbf{0}}\right) .
\end{aligned}
$$

Substituindo 2.6.20 em 2.6.19 e resolvendo,

$$
\mathbf{w}(k+1)=\mathbf{w}(k)-2 \eta \mathbf{R} \mathbf{w}+2 \eta \mathbf{R} \mathbf{w}^{\mathbf{0}}
$$

A análise da equação 2.6.21 é dificultada pelo acoplamento entre as componentes de w, de modo que é necessário transformar $\xi$ para um sistema de coordenadas principais através de duas transformaç̃ões isométricas. A primeira transformação consiste em uma operação de translação $\mathbf{v}=\mathbf{w}-\mathbf{w}^{\mathbf{0}}$,

$$
\begin{aligned}
\mathbf{w}(k+1) & =(\mathbf{I}-2 \eta \mathbf{R}) \mathbf{w}(k)+2 \eta \mathbf{R} \mathbf{w}^{\mathbf{0}} \\
\mathbf{w}(k+1)-\mathbf{w}^{\mathbf{0}} & =(\mathbf{I}-2 \eta \mathbf{R})\left(\mathbf{w}(k)-\mathbf{w}^{\mathbf{0}}\right) \\
\mathbf{v}(k+1) & =(\mathbf{I}-2 \eta \mathbf{R}) \mathbf{v}(k)
\end{aligned}
$$

seguida de uma operação de rotação $\mathbf{v}=\mathbf{Q} \mathbf{v}^{\prime}$, onde $\mathbf{Q}$ é a matriz que contém os autovetores $\mathbf{q}_{1}, \mathbf{q}_{2}, \ldots, \mathbf{q}_{N}$ de $\mathbf{R}$ respectivamente associados aos autovalores $\lambda_{1}, \lambda_{2}, \ldots, \lambda_{N}$, os quais são organizados em uma matriz de autovalores $\Lambda$, na qual todas as posições são nulas, exceto pela diagonal principal, isto é,

$$
\mathbf{Q}=\left[\begin{array}{llll}
\mathbf{q}_{1} & \mathbf{q}_{2} & \cdots & \mathbf{q}_{N}
\end{array}\right]
$$

e

portanto,

$$
\boldsymbol{\Lambda}=\left[\begin{array}{ccc}
\lambda_{1} & \cdots & 0 \\
\vdots & \ddots & \vdots \\
0 & \cdots & \lambda_{N}
\end{array}\right]
$$

$$
\mathbf{Q} \mathbf{v}^{\prime}(k+1)=(\mathbf{I}-2 \eta \mathbf{R}) \mathbf{Q} \mathbf{v}^{\prime}(k) .
$$


Multiplicando ambos os lados de 2.6 .25 pela matriz inversa de $\mathbf{Q}$

$$
\mathbf{v}^{\prime}(k+1)=\mathbf{Q}^{-1}(\mathbf{I}-2 \eta \mathbf{R}) \mathbf{Q} \mathbf{v}^{\prime}(k)
$$

e recordando a relação entre autovalores e autovetores

$$
\begin{aligned}
\mathrm{RQ} & =\mathrm{Q} \Lambda \\
\mathbf{R} & =\mathrm{Q} \Lambda \mathrm{Q}^{-1}
\end{aligned}
$$

a equação 2.6.26 pode, enfim, ser escrita na forma diagonal

$$
\mathbf{v}^{\prime}(k+1)=(\mathbf{I}-2 \eta \boldsymbol{\Lambda}) \mathbf{v}^{\prime}(k)
$$

A série 2.6.28 resultante da análise de convergência do algoritmo do gradiente descendente é estável e convergente desde que

$$
\lim _{k \rightarrow \infty}(\mathbf{I}-2 \eta \boldsymbol{\Lambda})^{k} \mathbf{v}^{\prime}(0)=\mathbf{0}
$$

isto é,

$$
\left[\begin{array}{llll}
\lim _{k \rightarrow \infty}\left(1-2 \eta \lambda_{0}\right)^{k} & & & \\
& & & \\
& \lim _{k \rightarrow \infty}\left(1-2 \eta \lambda_{1}\right)^{k} & & \\
& & \ddots & \\
& & \lim _{k \rightarrow \infty}\left(1-2 \eta \lambda_{N}\right)^{k}
\end{array}\right]=\mathbf{0}
$$

Tendo em vista que os autovalores da matriz de correlação $\mathbf{R}$ são todos reais e positivos, segue, portanto, que a condição necessária e suficiente para convergência ou estabilidade do algoritmo do gradiente descendente exige que o parâmetro da taxa de aprendizagem $\eta$ satisfaça a condição (22):

$$
0<\eta<\frac{1}{\lambda_{\max }}
$$

\subsubsection{Desenvolvimento do algoritmo LMS por meio do problema da filtragem adaptativa}

Supõe-se a princípio, que toda a informação disponível sobre determinado sistema de características desconhecidas esteja disponível sob a forma de um conjunto de dados de entradasaída $\mathcal{T}$ obtidos de maneira discretizada e também que ao ser estimulado por um sinal de 
entrada $\mathbf{x}(i) \in \Re^{m}$, onde $m$ é referido como dimensionalidade do espaço de entrada, este mesmo sistema responda de maneira que a saída seja um escalar $d(i)$. Deste modo, pode-se afirmar que o conjunto de dados

$$
\mathcal{T}:\{\mathbf{x}(i), d(i) ; i=1,2, \ldots, n\}
$$

descreve o comportamento deste sistema.

O algoritmo LMS sob o aspecto de um filtro adaptativo aplicado a identificação de sistemas visa obter iterativamente por meio de dois processos básicos, características desconhecidas que permitam descrever e prever o comportamento do sistema, estes processos são:

1. Processo de filtragem, o qual envolve o cálculo da saída $y(i)$ do filtro produzida por um vetor de coeficientes $\mathbf{w}$ e gera um estimativa de erro por meio da comparação desta saída com a resposta desejada $d(i)$.

2. Processo adaptativo, o qual envolve o ajuste dos coeficientes do filtro de acordo com a estimativa do erro.

Assim como no CLA, no algoritmo dos mínimos quadrados médios, o sinal de entrada $\mathbf{x}(i)$ e o sinal de saída $d(i)$ estão relacionados por meio da equação de erro 2.6 .5 , isto é,

$$
e(k)=d(k)-\mathbf{x}^{T}(k) \mathbf{w}(k)
$$

onde $\mathbf{w}(k) \in \Re^{m}$ é o chamado vetor de coeficientes ou parâmetros do filtro (equivalentes aos elementos ajustáveis vistos no (LA) no instante $k$. O algoritmo LMS modifica de maneira apropriada o vetor de coeficientes buscando minimizar uma função objeto dependente de w. $\mathrm{O}$ LMS pode ser interpretado como a versão estocástica do algoritmo do gradiente descendente visto anteriormente, tendo em vista que a função de custo $\xi$ definida neste como o valor quadrático médio do erro $e(k)$, grosso modo, é substituída pela estimativa instantânea do erro, isto é,

$$
\hat{\xi}(\mathbf{w})=\frac{e^{2}(n)}{2} .
$$

Fala-se em estimativa, pois geralmente junto aos dados de entrada $\mathbf{x}(1), \mathbf{x}(2), \ldots, \mathbf{x}(n) \mathrm{e}$ ao respectivo vetor de saída desejada $\mathbf{d}(k)$ estão associadas incertezas relacionadas ao processo de medida.

Tomando o gradiente da função de custo 2.6.33 em relação ao vetor de coeficientes

$$
\frac{\partial \hat{\xi}(\mathbf{w})}{\partial \mathbf{w}}=e(n) \frac{\partial e(n)}{\partial \mathbf{w}}
$$


e resolvendo a derivada parcial do lado direito da equação

$$
\frac{\partial e(n)}{\partial \mathbf{w}}=-\mathbf{x}(n)
$$

chega-se a estimativa do vetor gradiente para a função de custo $\hat{\xi}(\mathbf{w})$, isto é,

$$
\frac{\partial \hat{\xi}(\mathbf{w})}{\partial \mathbf{w}}=-\mathbf{x}(n) e(n)
$$

surge daí, portanto, a denominação algoritmo de gradiente estocástico. Por fim, substituindo 2.6.36 na equação recursiva do método do gradiente descendente 2.6.18 completa-se a formulação comum do algoritmo dos mínimos quadrados médios, isto é,

$$
\hat{\mathbf{w}}(n+1)=\hat{\mathbf{w}}(n)+\eta \mathbf{x}(n) e(n)
$$

A análise de convergência do vetor de parâmetros do LMS na média, supondo independência estatística entre os elementos $\mathbf{x}(k)$ e $\mathbf{v}(k)$ e $\mathbf{x}(k)$ e $e^{0}$ é idêntica ao que foi demonstrado para o CLA, isto é, a convergência do algoritmo é garantida desde que

$$
0<\eta<\frac{1}{\lambda_{\max }}
$$

A condição de estabilidade anterior conforme discutido e demonstrado em (23) pode ser substituída por

$$
0<\eta<\frac{1}{2 \lambda_{\max }+\sum_{i=0}^{N} \lambda_{i}}<\frac{1}{\sum_{i=0}^{N} \lambda_{i}}=\frac{1}{\operatorname{tr}[\mathbf{R}]}
$$

onde a última expressão é mais utilizada na prática por ser mais simples de estimar tendo em vista que está relacionada com o quadrado da norma euclideana do sinal de entrada.

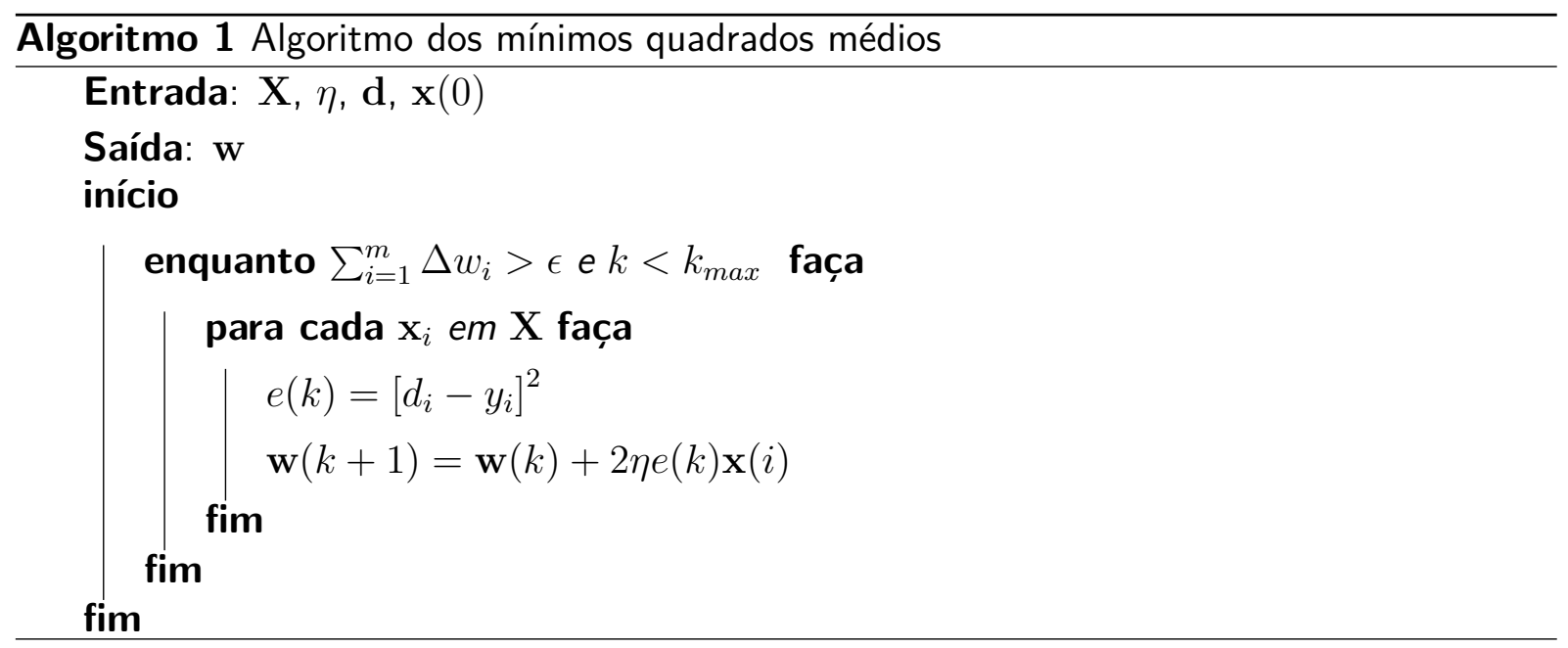




\subsubsection{Obtendo distribuições $T_{2}$ com LMS}

Considerando distribuição de valores $T_{1}$ ou $T_{2}$ simuladas através de distribuições multilog-normal dada por:

$$
g\left(\Gamma_{i}\right)=g\left(T_{1}\right)=g\left(T_{2}\right)=\sum_{k=1}^{N} A_{k} \exp \left(-\sigma_{k} \log \left(\frac{\Gamma_{i}}{T_{c_{k}}}\right)^{2}\right)
$$

onde os $A_{k}$ são as $n$ amplitudes máximas das distribuições localizadas nos centros $T_{c_{k}}$ com espalhamento de valores $\sigma_{k}$. Os respectivos sinais de magnetização simulados formados pelas distribuições são descritos através das equações multi exponenciais:

$$
\begin{gathered}
M_{\perp}\left(\tau_{j}^{*}\right)=\sum_{i=1}^{n} g\left(\Gamma_{i}\right) \exp \left(-\frac{\tau_{j}^{*}}{\Gamma_{i}}\right)+\epsilon_{j}, j=1,2, \ldots, m . \\
M_{\|}\left(\tau_{j}^{*}\right)=\sum_{i=1}^{n} g\left(\Gamma_{i}\right)\left(1-\exp \left(\frac{\tau_{j}^{*}}{\Gamma_{i}}\right)\right)+\epsilon_{j}, j=1,2, \ldots, m .
\end{gathered}
$$

onde $\tau_{j}^{*}=2 \tau$ e $\epsilon_{j}$ é uma componente de ruído somada ao sinal de magnetização simulado a fim de torná-lo mais próximo daqueles obtidos experimentalmente.

Figura 2.15 - Distribuição multi-log-normal composta por 4096 amostras logaritmomanicamente espaçadas no intervalo $\left[10^{-5}, 10^{-2}\right]$, com $A_{1}=0,8$ e $A_{2}=0,2, T_{c_{1}}=10^{-3} \mathrm{e}$ $T_{c_{2}}=10^{-1}$ e $\sigma_{1}=2,5$ e $\sigma_{2}=1$

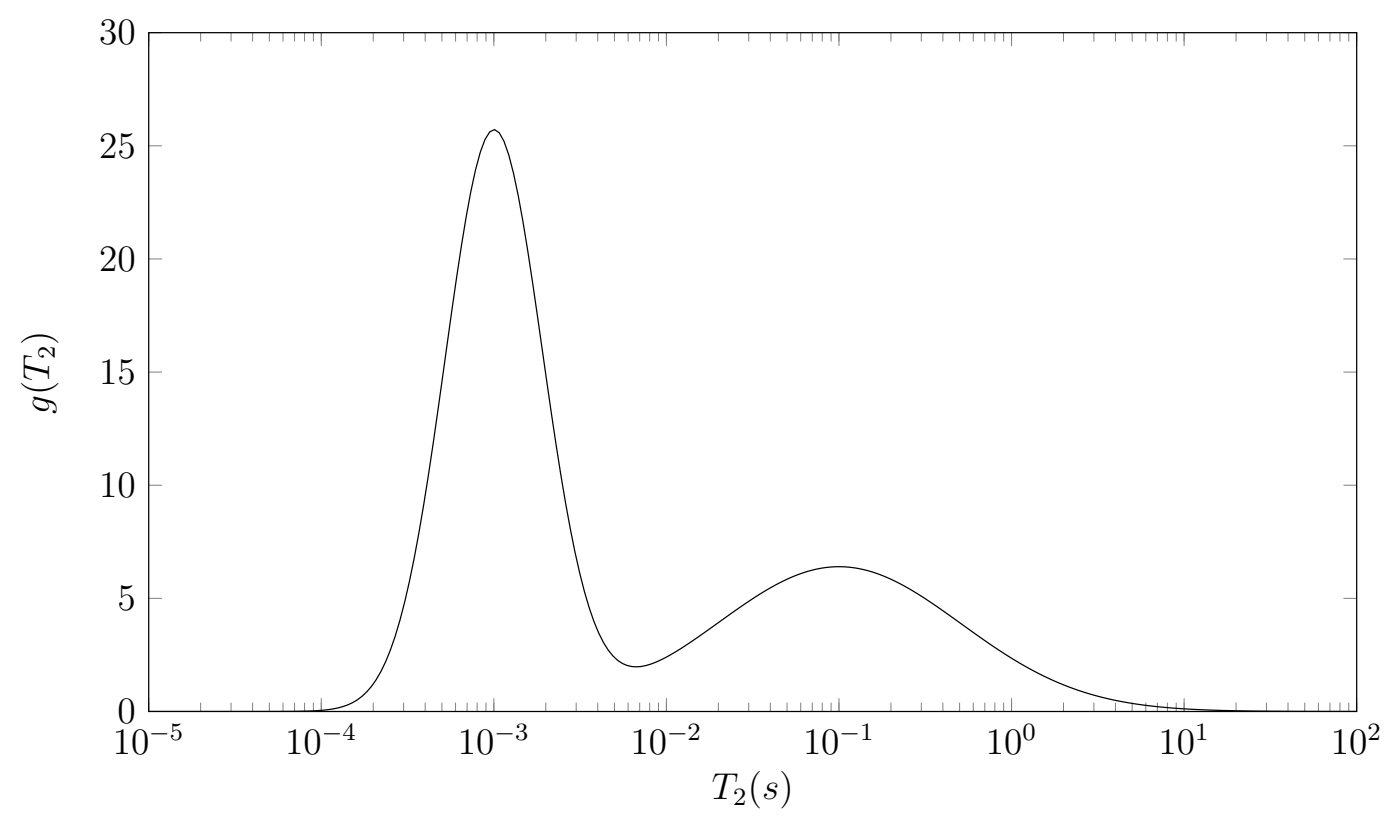

Fonte: Elaborada pelo autor. 
Figura 2.16 - Decaimento multi exponencial composto por 1024 amostras, contaminadas com ruído gaussiano, para a distribuição da figura 2.15 , com diversos tempos de aquisição.

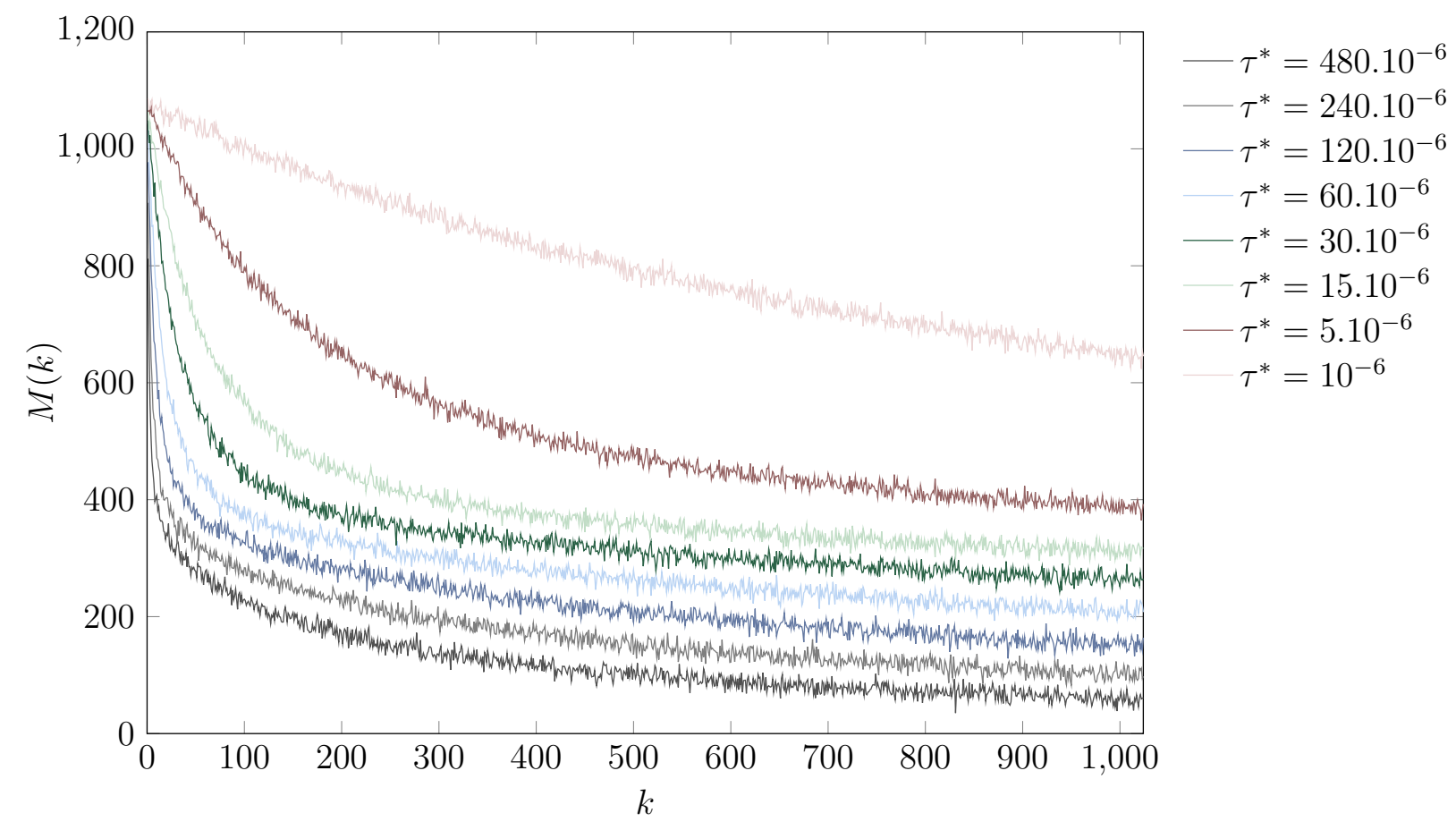

Fonte: Elaborada pelo autor.

Figura 2.17 - Distribuição log-normal composta por 4096 amostras logaritmomanicamente espaçadas no intervalo $\left[10^{-5}, 10^{-2}\right]$, com $A_{1}=0,8, T_{c_{1}}=10^{-2}$ e $\sigma_{1}=2,5$.

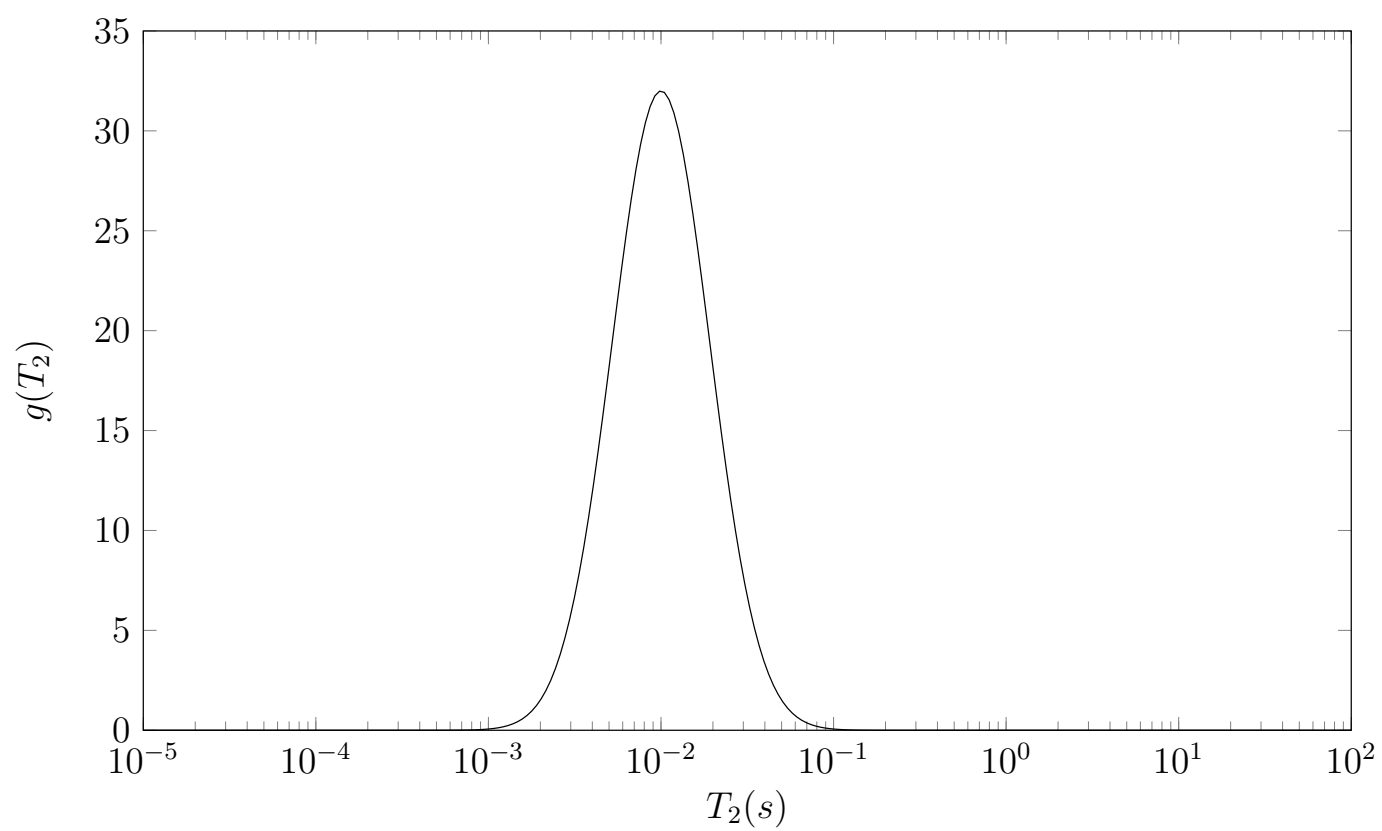

Fonte: Elaborada pelo autor. 
Figura 2.18 - Decaimento multi exponencial composto por 1024 amostras, contaminadas com ruído gaussiano, para a distribuição da figura 2.17, com diferentes tempos de eco.

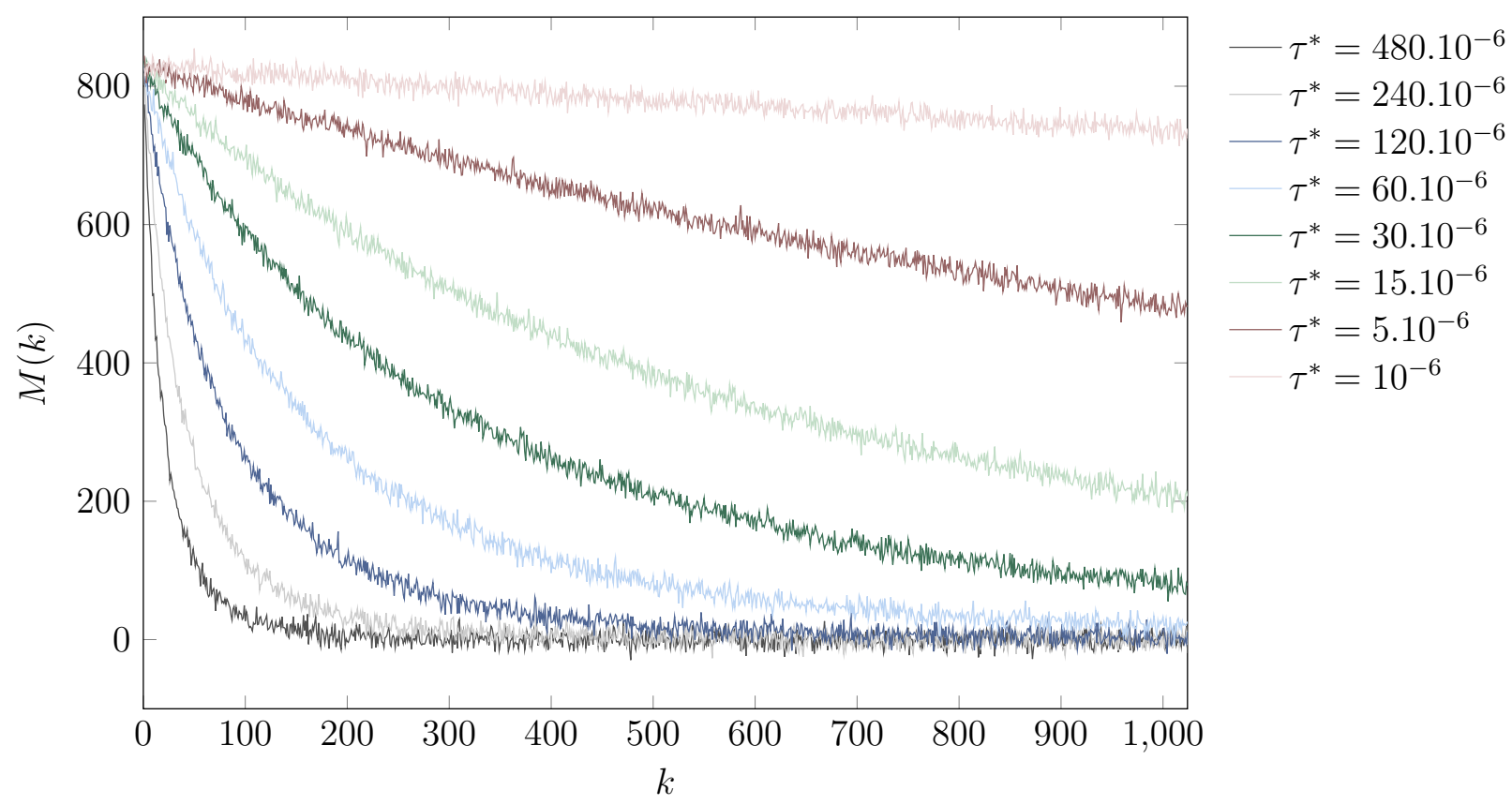

Fonte: Elaborada pelo autor.

Reescrevendo $M_{\perp}$ e $M_{\|}$na forma geral

$$
M\left(\tau_{j}^{*}\right)=\sum_{i=1}^{n} g\left(\Gamma_{i}\right) K\left(\tau_{j}^{*}, \Gamma_{i}\right)+\epsilon_{j}, j=1,2, \ldots, m
$$

ou ainda $M\left(\tau_{j}^{*}\right)$ na forma matricial

$$
M\left(\tau_{j}^{*}\right)=\mathbf{K}^{T} \mathbf{g}+\epsilon
$$

onde $\mathbf{K} \in \Re^{m \times n}$ é a matriz que contém os $m$ sinais de entrada, isto é, cada linha de $\mathbf{K}$ corresponde a um sinal de entrada de dimensão $n$ formado pelos núcleos:

$$
K\left(\tau^{*}, \Gamma_{1}\right), K\left(\tau^{*}, \Gamma_{2}\right), \ldots, K\left(\tau^{*}, \Gamma_{n}\right)
$$

isto é,

$$
\mathbf{K} \triangleq \mathbf{X}=\left[\begin{array}{ccc}
K\left(\tau_{1}^{*}, \Gamma_{1}\right) & \cdots & K\left(\tau_{1}^{*}, \Gamma_{n}\right) \\
\vdots & \ddots & \vdots \\
K\left(\tau_{m}^{*}, \Gamma_{1}\right) & \cdots & K\left(\tau_{m}^{*}, \Gamma_{n}\right)
\end{array}\right]
$$

$\mathbf{m} \in \Re^{m}$ é o vetor que contém os respectivos sinais de saída, ou respostas desejadas,

$$
\mathbf{m} \triangleq \mathbf{d}=\left[\begin{array}{llll}
M\left(\tau_{1}^{*}\right) & M\left(\tau_{2}^{*}\right) & \cdots & M\left(\tau_{m}^{*}\right)
\end{array}\right]^{T}
$$


e $\mathrm{g} \in \Re^{n}$ é o vetor que contém os parâmetros do filtro a serem identificados, isto é, a distribuição de valores $T_{1}$ ou $T_{2}$,

$$
\mathbf{g} \triangleq \mathbf{w}=\left[\begin{array}{llll}
g\left(\Gamma_{1}\right) & g\left(\Gamma_{2}\right) & \cdots & g\left(\Gamma_{n}\right)
\end{array}\right]^{T}
$$

É possível notar nos gráficos dos sinais simulados 2.16 e 2.18 o efeito da filtragem comentado no fim do capítulo 2, consequência da integração envolvendo os núcleos $K\left(\tau^{*}, T_{1}\right)$ e $K\left(\tau^{*}, T_{2}\right)$ nas equações que descrevem as magnetizações. O efeito suavizante pode ser visto também como reflexo das características da função exponencial que compõe os núcleos, isto é, conforme o argumento da função exponencial cresce mais rapidamente (em comparação ao tamanho da amostra), para a relaxação transversal, por exemplo, as contribuições dos decaimentos mono exponenciais dos $T_{2}$ menores praticamente dominam o sinal $M\left(\tau^{*}\right)$, no caso oposto, isto é, quando o argumento da função exponencial decresce rapidamente, a composição de $M\left(\tau^{*}\right)$ é dominada pelas contribuições dos $T_{2}$ maiores.

A função objeto a ser minimizada pelo LMS é dada por:

$$
\begin{aligned}
\xi(\mathbf{g}(k)) & =e(k)^{2} \\
& =\left[M\left(\tau_{j}^{*}\right)-\sum_{i=1}^{n} K\left(\tau_{j}^{*}, \Gamma_{i}\right) g_{i}(k)\right]^{2}
\end{aligned}
$$

onde $k$ representa a $k$-ésima iteração do algoritmo. Os $m$ sinais de entrada e os respectivos sinais de saída são apresentados aleatoriamente ao algoritmo até que ocorra convergência, isto é, enquanto $\|\Delta \mathbf{w}\|^{2}>\epsilon$ ou enquanto o número de iterações $k$ não atingir um limite imposto $k_{\text {max }}$.

A equação de atualização dos parâmetros do filtro, assim como exposto anteriormente, é obtida substituindo a função objeto 2.6 .47 no algoritmo do gradiente descendente, isto é,

$$
\begin{gathered}
\boldsymbol{\Delta} \mathbf{w}=-\eta \frac{\partial e(k)^{2}}{\partial \mathbf{w}(k)} \\
w_{i}(k+1)=w_{i}(k)+2 \eta K\left(\tau_{j}^{*}, \Gamma_{i}\right) e(k), i=1,2, \ldots, n .
\end{gathered}
$$

Infelizmente, por si só, o algoritmo LMS não garante não-negatividade na solução, característica desejável quando a resposta esperada corresponde a uma distribuição de valores, de modo que é necessário impor ao algoritmo LMS restrições na busca do vetor de parâmetros g. 


\subsubsection{Algoritmo LMS com restrição de não-negatividade (LMS-NN)}

Frequentemente a estimação de parâmetros de determinados sistemas conduz a necessidade de impor restrições na busca por soluções. A determinação das distribuições de tempos de relaxação $T_{1}$ e $T_{2}$ em sinais obtidos em experimentos de RMN envolvendo meios porosos é um destes casos. Do exposto na seção 2.4 sabe-se que as distribuições de valores $g\left(T_{1}\right)$ e $g\left(T_{2}\right)$ podem ser associadas ao espectro de tamanhos de poros da amostra em estudo, de modo que $g\left(T_{1}\right)<0$ ou $g\left(T_{2}\right)<0$ para qualquer $T_{1}$ ou $T_{2}$ não possui significado físico (24), portanto a restrição de não negatividade na inversão dos sinais de magnetização deve conduzir o algoritmo LMS à uma solução mais coerente.

O método proposto a seguir combina o algoritmo LMS com as chamadas condições de Karush-Kuhn-Tucker (KKT).

Partindo do problema de identificar o modelo ótimo

$$
\begin{gathered}
\mathbf{g}^{0}=\underset{\mathbf{g}}{\arg \min } \xi(\mathbf{g}) \\
\text { sujeito a } g_{i} \geq 0, \forall i
\end{gathered}
$$

onde $\xi(\mathrm{g})$ é a mesma função objeto 2.6.47. Na busca de uma solução para 2.6.51 considera-se a função Langrangeana $Q(\mathbf{w}, \boldsymbol{\alpha})$ dada por $(25)(26)$ :

$$
Q(\mathbf{g}, \boldsymbol{\alpha})=\xi(\mathbf{g})-\boldsymbol{\alpha}^{T} \mathbf{g}
$$

onde $\boldsymbol{\alpha}$ é o vetor não negativo que contém os chamados multiplicadores de Lagrange.

Os valores ótimos $\mathrm{g}^{0}$ e $\boldsymbol{\alpha}^{0}$ obrigatoriamente devem satisfazer as seguintes condições de Karush-Kuhn-Tucker:

$$
\begin{aligned}
\nabla_{\mathbf{g}} Q\left(\mathbf{g}^{0}, \boldsymbol{\alpha}^{0}\right) & =0 \\
g_{i}^{0}\left[\boldsymbol{\alpha}^{0}\right] & =0, \forall i
\end{aligned}
$$

Onde $\nabla_{\mathbf{g}} Q(\mathbf{g}, \boldsymbol{\alpha})$ é o gradiente da função Lagrangeana em relação ao vetor $\mathbf{g}$, isto é,

$$
\nabla_{\mathbf{g}} Q(\mathbf{g}, \boldsymbol{\alpha})=\frac{\partial Q\left(\mathbf{g}^{0}, \boldsymbol{\alpha}^{0}\right)}{\partial \mathbf{g}}
$$

Tomando o gradiente do lado direito de 2.6.52,

$$
\nabla_{\mathbf{g}} Q(\mathbf{g}, \boldsymbol{\alpha})=\nabla_{\mathbf{g}} \xi(\mathbf{g})-\boldsymbol{\alpha}
$$


e considerando a condição 2.6 .53

$$
\nabla_{\mathbf{g}} \xi(\mathbf{g})=\boldsymbol{\alpha}
$$

as condições de KKT podem ser combinadas na seguinte expressão (27)(28)

$$
g_{i}^{0}\left[-\nabla_{\mathbf{g}} \xi\left(\mathbf{g}^{0}\right)\right]=0
$$

O sinal negativo especifica a busca na direção oposta ao gradiente.

Solucionando 2.6.57 por meio do algoritmo do gradiente descendente, a atualização dos parâmetros do filtro é dada por:

$$
g_{i}(k+1)=g_{i}(k)+2 \eta K\left(\tau_{j}^{*}, \Gamma_{i}\right) e(k) g_{i}(k) .
$$

Assumindo que $g_{i}(k) \geq 0$, então $g_{i}(k+1) \geq 0$ para qualquer $\eta_{i} \geq 0$ se $2 K\left(\tau_{j}^{*}, \Gamma_{i}\right) e(k) \geq 0$. Para $2 K\left(\tau_{j}^{*}, \Gamma_{i}\right) e(k)<0$ a positividade de $g_{i}(k+1)$ é satisfeita para qualquer

$$
\eta_{i} \leq \frac{1}{2 K\left(\tau_{j}^{*}, \Gamma_{i}\right) e(k)}
$$

Portanto, a positividade de $g_{i}(k+1)$ é garantida, em todo caso, se,

$$
\begin{gathered}
g_{i}(0)>0, \forall i \\
0 \leq \eta_{i} \leq \frac{1}{2 K\left(\tau_{j}^{*}, \Gamma_{i}\right) e(k)}
\end{gathered}
$$

A equação de atualização dos pesos do filtro 2.6 .58 pode também ser escrita na forma vetorial:

$$
\mathbf{g}(k+1)=\mathbf{g}(k)+2 \eta(k) e(k) \mathbf{D}(k) \mathbf{k}(k),
$$

onde $\mathbf{D}(k)$ é uma matriz diagonal composta pelos parâmetros $\mathbf{g}(k)$ do filtro e $\mathbf{k}(k)$ é o sinal de entrada avaliado na iteração $k$. O parâmetro taxa de aprendizagem $\eta(k)$ é fixado em cada iteração com um valor no intervalo $\left[0, \eta_{\max }(k)\right]$ onde,

$$
\eta_{\text {max }}(k)=\min _{i}\left\{\frac{1}{2 K\left(\tau_{j}^{*}, \Gamma_{i}\right) e(k)}\right\} \text { para } i=1,2, \ldots n
$$




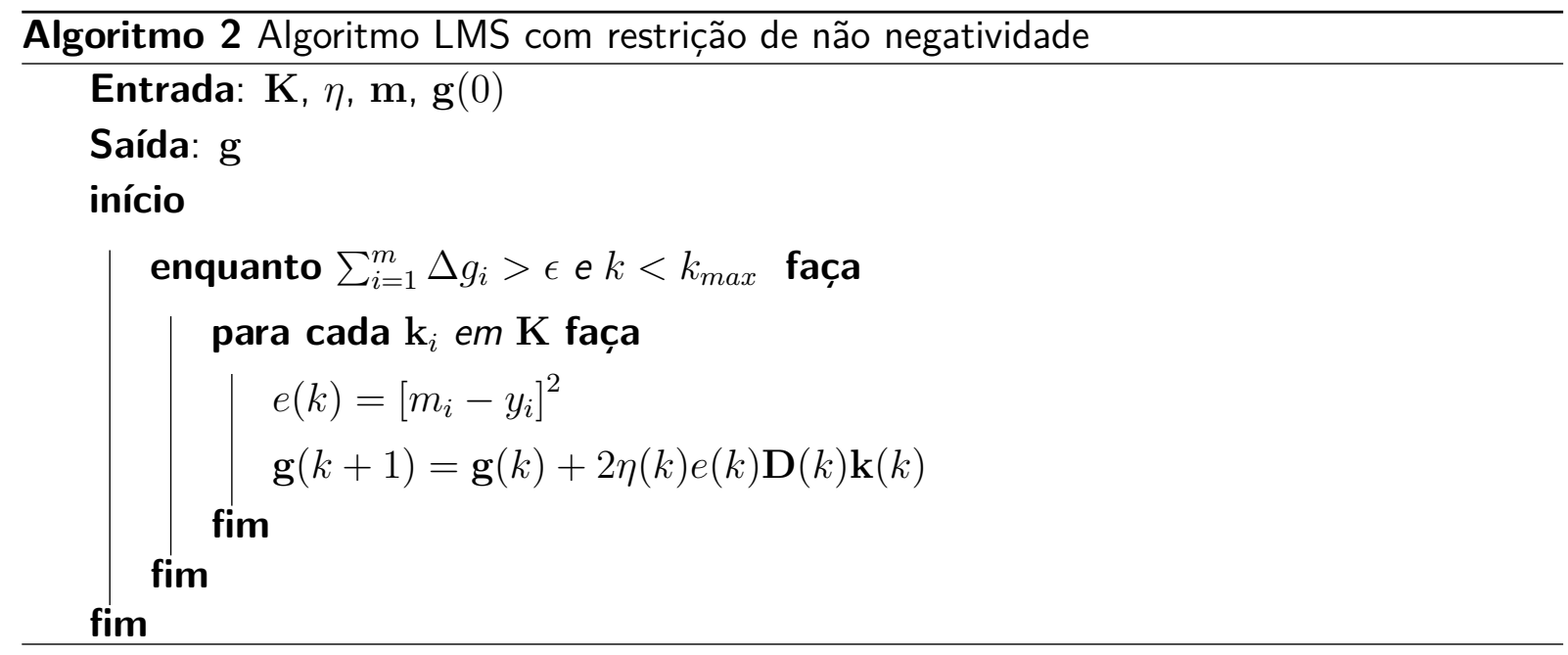

\subsubsection{Algoritmo LMS regularizado e não-negativo (LMS-RNN)}

A teoria da regularização é aplicada com frequência na solução de problemas inversos malpostos devido a necessidade de incorporar informação adicional acerca da solução procurada visando estabilizar os problemas e de modo a encontrar soluções úteis e coerentes. Dentre os métodos utilizados na regularização de problemas um dos mais célebres é a regularização de Tikhonov, desenvolvido independentemente por A. Tikhonov (29) e D. Phillips (30). A ideia básica por trás do método é supor que a solução procurada é suave. A suposição de suavidade é escrita na forma de uma semi-norma $\lambda\|\mathbf{L g}\|^{2}$, onde $\lambda \geq 0$ é o parâmetro que controla o peso dado na minimização para o termo de regularização e $\mathbf{L}$ é a aproximação de um operador derivativo de segunda ordem dado por:

$$
\mathbf{L}=\left[\begin{array}{ccccc}
1 & -2 & 1 & & \\
& \ddots & \ddots & \ddots & \\
& & 1 & -2 & 1
\end{array}\right] \in \Re^{(n-2) \times n}
$$

O algoritmo LMS com restrição de não-negatividade e com regularização de Tikhonov é obtido então a partir do seguinte problema de otimização:

$$
\begin{array}{r}
\min _{\mathbf{g}}\left\{\xi(\mathbf{g})+\lambda\|\mathbf{L g}\|^{2}\right\} \\
\text { sujeito a } g_{i} \geq 0, \forall i
\end{array}
$$

Substituindo 2.6.61 no algoritmo do gradiente descendente e conduzindo conforme na sessão anterior obtém-se a equação de atualização dos pesos para o LMS-RNN: 


$$
\begin{gathered}
\mathbf{\Delta} \mathbf{g}=-\eta \frac{\partial \xi(\mathbf{g})+\lambda \mathbf{L}^{T} \mathbf{g g}^{T} \mathbf{L}}{\partial \mathbf{g}} \\
\mathbf{g}(k+1)=\mathbf{g}(k)+2 \eta(k) \mathbf{D}(k)\left[\mathbf{k}(k) e(k)-\lambda \mathbf{L}^{T} \mathbf{L} \mathbf{g}(k)\right]
\end{gathered}
$$

onde a positividade de $\mathbf{g}(k+1)$ é garantida para

$$
\begin{gathered}
g_{i}(0)>0, \forall i \\
0 \leq \eta(k) \leq \frac{1}{2 \eta_{\min }(k)},
\end{gathered}
$$

onde $\eta_{\text {min }}(k)$ é a menor componente do vetor definido por $\mathbf{k}(k) e(k)-\lambda \mathbf{L}^{T} \mathbf{L} \mathbf{g}(k)$.

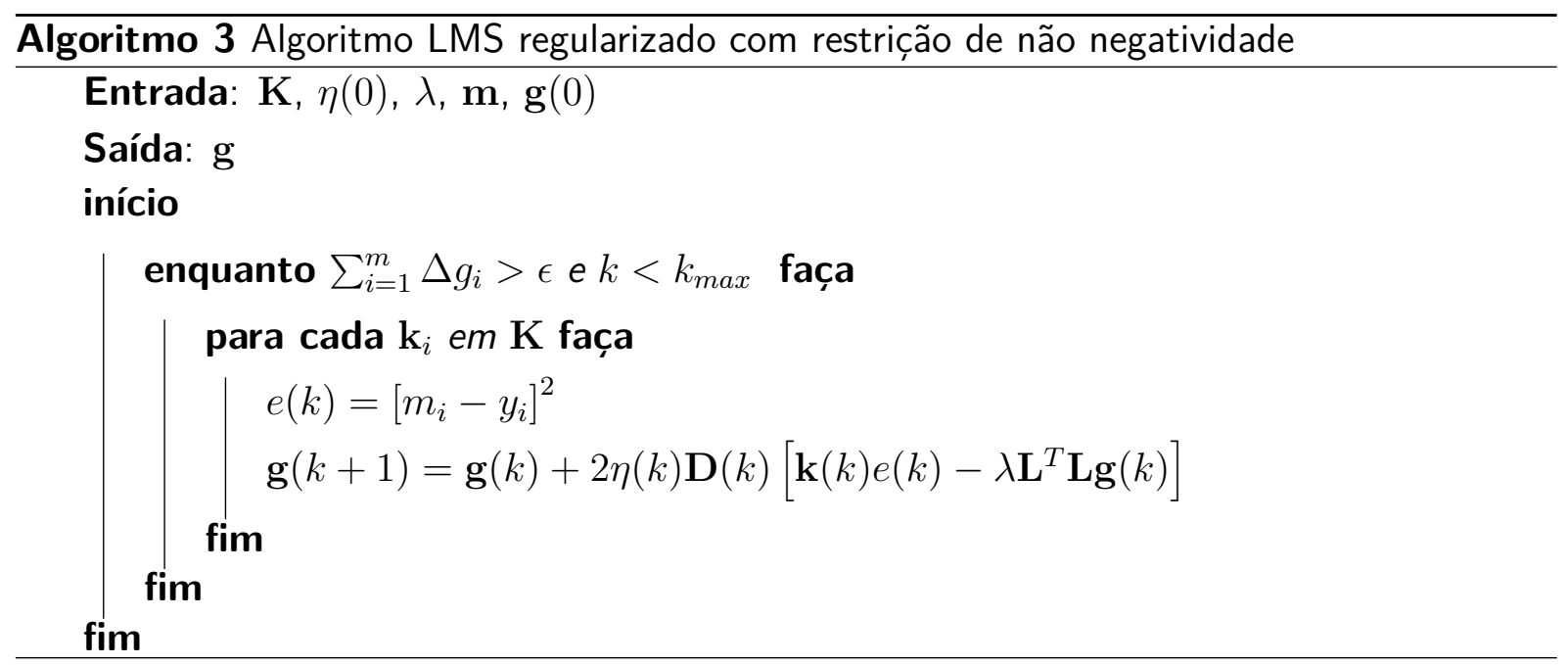

\subsection{Redes recorrentes de Hopfield}

Sabe-se que o cérebro humano funciona como um computador extremamente complexo, não-linear e altamente paralelo, reflexo de duas características principais: o processamento é distribuído por um número grande de unidades simples de processamento, os neurônios, e a capacidade de auto-organização destas unidades. As redes neurais artificiais, de maneira geral, tentam modelar a forma com que o cérebro biológico realiza uma tarefa, o processamento é realizado por neurônios artificiais (modelo simplificado do neurônio biológico, ver figura 2.19), os quais possuem a capacidade de aprender através da experiência por meio de algoritmos conhecidos como algoritmos de treinamento.

As redes neurais artificiais diferem entre si por duas características básicas, a topologia e a arquitetura. A topologia define a quantidade e o modo de operação dos neurônios, enquanto 
Figura 2.19 - Unidades responsáveis pelo processamento nas redes neurais.

a)Esquema simplificado de um neurônio biológico.

b)Neurônio artificial típico

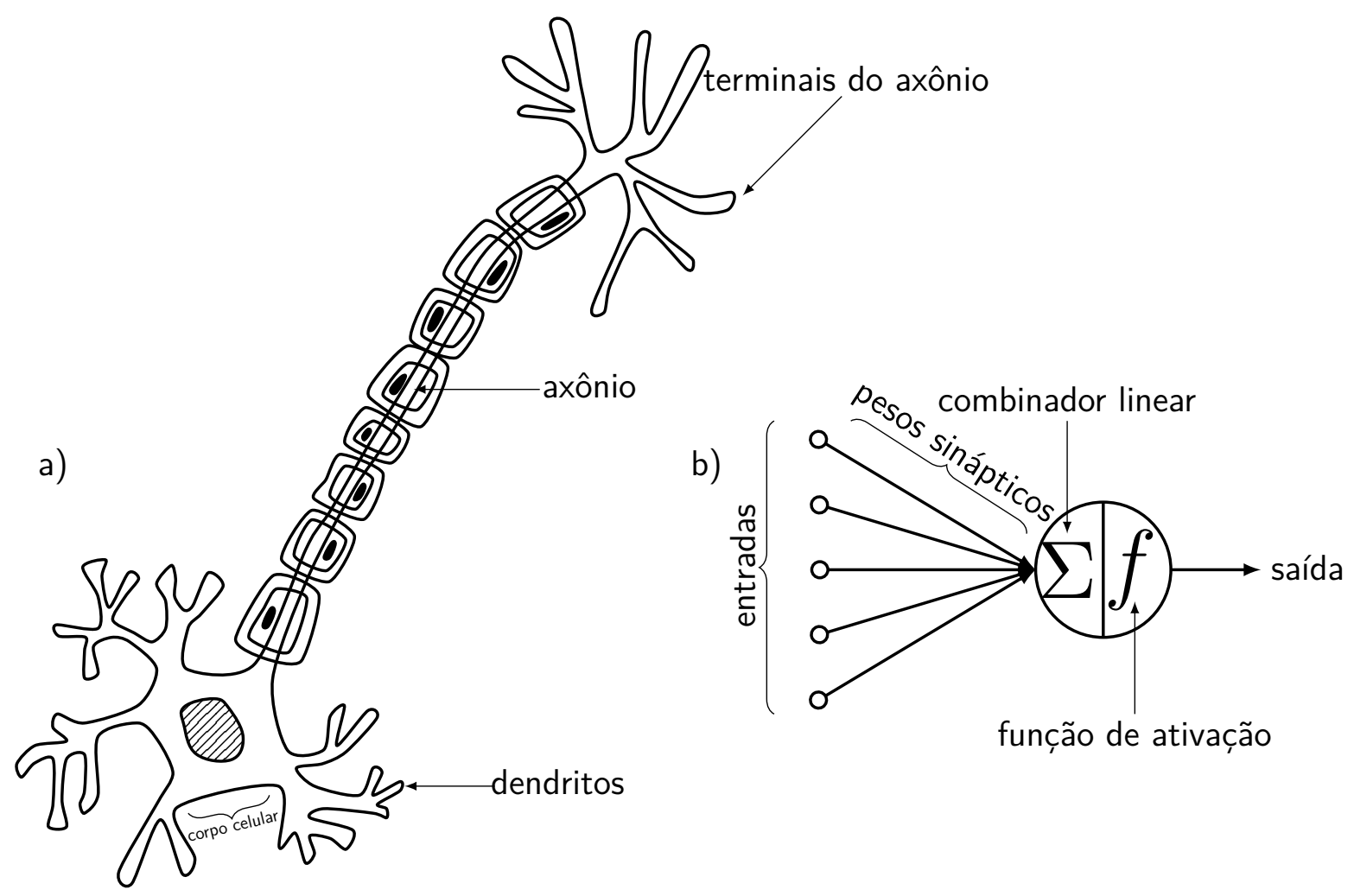

Fonte: Elaborada pelo autor.

a arquitetura está relacionada com a disposição dos neurônios e com o sentido de propagação dos sinais na rede, isto é, define se o sinal percorre a rede em apenas um sentido, as chamadas redes com alimentação adiante (feedforward), ou se existe algum tipo de realimentação, redes conhecidas como recorrentes. (31)

As redes recorrentes de Hopfield são constituídas basicamente por um conjunto de unidades de processamento, isto é, os neurônios artificiais, os quais estão organizados em uma única camada e um conjunto correspondente de atrasos unitários. As unidades de processamento da rede são completamente interligadas, isto é, todos os neurônios estão conectados a todos os outros. Nas redes de Hopfield discretas convencionais normalmente não há auto retroalimentação. Esta característica de recorrência confere a rede um comportamento tipicamente dinâmico descrito por um modelo conhecido como modelo neurodinâmico. O estudo das redes neurais vistas como sistemas dinâmicos não-lineares, com enfase particular no problema da estabilidade, é referido como neurodinâmica.

Quando se fala em estabilidade no contexto das redes neurais, devido a saturação da nãolinearidade que compõe o neurônio artificial, refere-se estabilidade no sentido do método direto 
Figura 2.20 - Rede de Hopfield convencional.

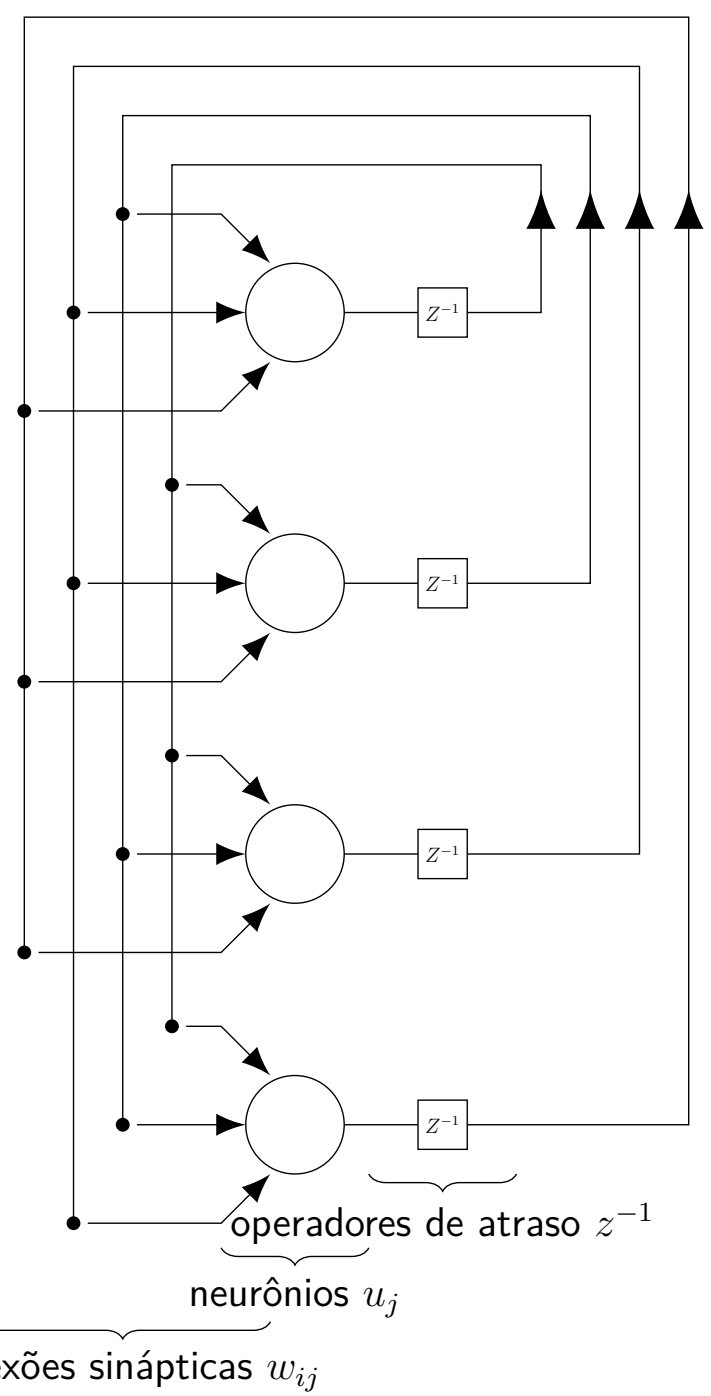

Fonte: Elaborada pelo autor.

de Lyapunov (também conhecido como segundo método de Lyapunov) e não pelo critério normalmente adotado de entrada limitada - saída limitada. O método direto de Lyapunov investiga o problema da estabilidade sem a necessidade de resolver equações de espaço de estados dos sistemas dinâmicos. Realiza-se a análise de estabilidade por meio de uma função escalar contínua $V(\mathbf{x})$ do vetor de estado $\mathbf{x}(t)$, conhecida como função de Lyapunov, isto é, seja o sistema não-linear descrito pelo sistema de equações diferenciais de primeira ordem,

$$
\frac{d \mathbf{x}(t)}{d t}=\mathbf{F}(\mathbf{x}(t))
$$

os teoremas de Lyapunov podem ser aplicada sem a necessidade de resolver 2.7.1, desde que a função $V(\mathbf{x})$ satisfaça as seguintes condições: 
- $V(\mathbf{x})$ deve possuir derivadas continuas em relação aos elementos do vetor de estado.

- $V(\overline{\mathbf{x}})=0$, onde $\overline{\mathbf{x}}$ é um estado de equilíbrio.

- $V(\mathbf{x})>0$ se $\mathbf{x} \neq \overline{\mathbf{x}}$.

De posse da função de Lyapunov $V(\mathbf{x})$ o estado de equilíbrio $\overline{\mathbf{x}}$ é estável se

$$
\frac{d V(\mathbf{x})}{d t} \leq 0 \text { para } \quad \mathbf{x} \in \mathcal{U}-\overline{\mathbf{x}}
$$

onde $\mathcal{U}$ é uma pequena vizinhança de $\overline{\mathbf{x}}$, ou ainda, $\overline{\mathbf{x}}$ é assintoticamente estável se

$$
\frac{d V(\mathbf{x})}{d t}<0 \text { para } \quad \mathbf{x} \in \mathcal{U}-\overline{\mathbf{x}}
$$

Infelizmente encontrar a função de Lyapunov é um problema a parte, contudo, em muitos casos, a função de energia, conforme demonstrado por Hopfield em (33) e (34), pode servir como função de Lyapunov.

\subsubsection{Funcionamento da rede de Hopfield}

A expressão simplificada que rege o comportamento de cada neurônio $u_{j}$ do diagrama 2.20 é dada por:

$$
\begin{gathered}
u_{j}=\sum_{i=1}^{N} w_{i j} v_{i}+I_{j} \text { para } j=1,2, \ldots, N . \\
v_{j}=\phi\left(u_{j}\right)
\end{gathered}
$$

onde $v_{j}$ é a saída do $j$-ésimo neurônio, $w_{i j}$ é a força da conexão sináptica entre os neurônios $i$ e $j, I_{j}$ é o bias aplicado ao neurônio $j$ e $\phi\left(u_{j}\right)$ é uma função monótona crescente, tipicamente a função logística, definida como:

$$
\phi\left(u_{j}\right)=\frac{1}{1+\exp \left(-\alpha u_{j}\right)}
$$

ou a função tangente hiperbólica, dada por:

$$
\phi\left(u_{j}\right)=\frac{1-\exp \left(-\alpha u_{j}\right)}{1+\exp \left(-\alpha u_{j}\right)} .
$$

Para um grande número de neurônios o modelo descrito anteriormente pode apresentar 
estruturas de atratores complicadas, que é exatamente a ideia por trás das redes recorrentes de Hopfield, a manipulação dessas estruturas através dos algoritmos de treinamento sob a forma de equações dinâmicas pode permitir a codificação de informação ou mesmo de extração de informações sobre relacionamentos de entrada-saída.

Impondo uma condição de simetria para a matriz de pesos $\mathbf{W}$, através da função de energia definida por Hopfield como:

$$
E=-\frac{1}{2} \mathbf{v}^{T} \mathbf{W} \mathbf{v}-\mathbf{v}^{T} \mathbf{I}
$$

e da respectiva derivada temporal

$$
\frac{d E}{d t}=\left(\frac{\partial E}{\partial \mathbf{v}}\right)^{T} \frac{d \mathbf{v}}{d t}
$$

chega-se a seguinte relação:

$$
\frac{\partial E}{\partial \mathbf{v}}=-\frac{d \mathbf{u}}{d t}
$$

Substituindo 2.7.10 em 2.7.9 Hopfield demonstrou que as derivadas temporais da função de energia serão sempre menores ou iguais à zero, isto é, dado qualquer conjunto de condições iniciais a rede de Hopfield sempre convergirá para um ponto de equilíbrio estável.

\subsubsection{Obtendo distribuições $T_{1}, T_{2}$ com as redes de Hopfield}

Considerando novamente as distribuições de valores $T_{1}$ ou $T_{2}$ simuladas por meio de distribuições multi log-normal dadas por 2.6 .39 e os respectivos sinais de magnetização simulados $M\left(\tau_{j}^{*}\right)$ descritos por 2.6.42, pode-se definir a seguinte função de energia para a rede de Hopfield (35):

$$
E=\frac{1}{2} \sum_{j=1}^{m} e_{j}^{2}=\frac{1}{2} \sum_{j=1}^{M}\left(\left(\sum_{i=1}^{N} K_{i j} v_{i}\right)-m_{j}\right)^{2},
$$

onde $K_{i j}$ é o núcleo $K\left(\tau_{j}^{*}, \Gamma_{i}\right)$ e $m_{j}$ é o sinal de magnetização $M\left(\tau_{j}^{*}\right)$.

Reescrevendo a relação 2.7 .10 como:

$$
\frac{d \mathbf{u}}{d t}=-\frac{\partial E}{\partial \mathbf{v}}
$$

substituindo 2.7.11 no lado direito de 2.7 .12 e resolvendo, chega-se em:

$$
\frac{d u_{i}}{d t}=-\sum_{j=i}^{M}\left(\sum_{i=1}^{N} K_{i j} v_{j}-m_{j}\right) K_{j i} .
$$


Comparando 2.7 .13 e 2.7.4 identifica-se as seguintes relações (36):

$$
\begin{gathered}
w_{i j}=\sum_{l=1}^{n} K_{l i} K_{l j}=w_{j i}, \\
I_{i}=\sum_{j=1}^{n} K_{j i} m_{j} \\
v_{i}=\phi\left(u_{i}\right) .
\end{gathered}
$$

onde $\phi\left(u_{i}\right)$ é dado pela função logística 2.7.6.

A equação 2.7.13 deve ser integrada até que alcance o equilíbrio, o qual corresponde a solução $\mathbf{v}$ do problema, isto é, $\mathbf{v}$ corresponde a distribuição de valores $T_{1}$ ou $T_{2}$ para um dado sinal de magnetização $M\left(\tau_{j}^{*}\right)$.

Do gráfico de $\phi\left(u_{i}\right)$ abaixo nota-se que conforme esperado a solução $\mathbf{v}$ correspondente a distribuição dos tempos de relaxação é sempre maior ou igual a zero.

Figura 2.21 - Gráfico da função logística.

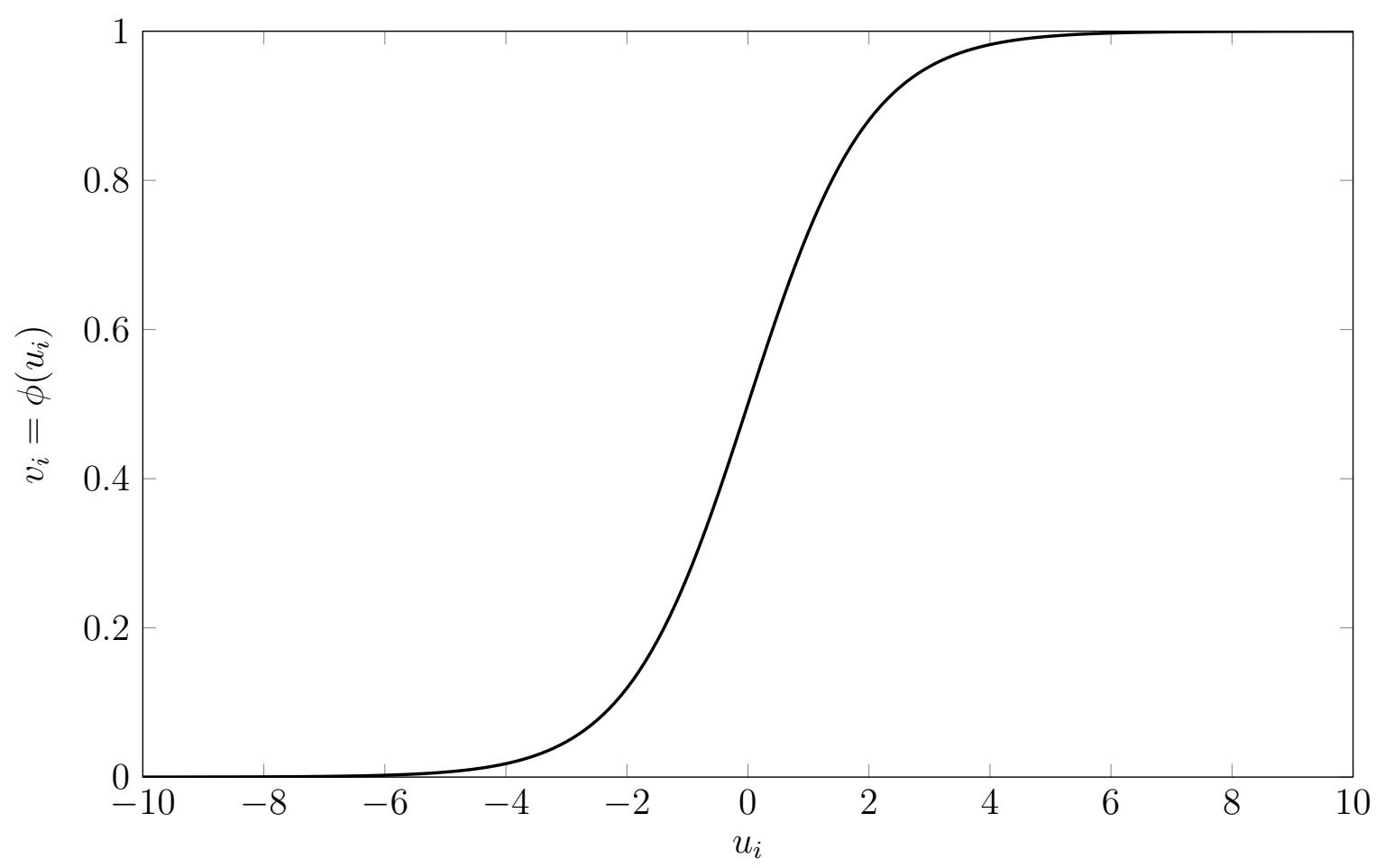

Fonte: Elaborada pelo autor. 


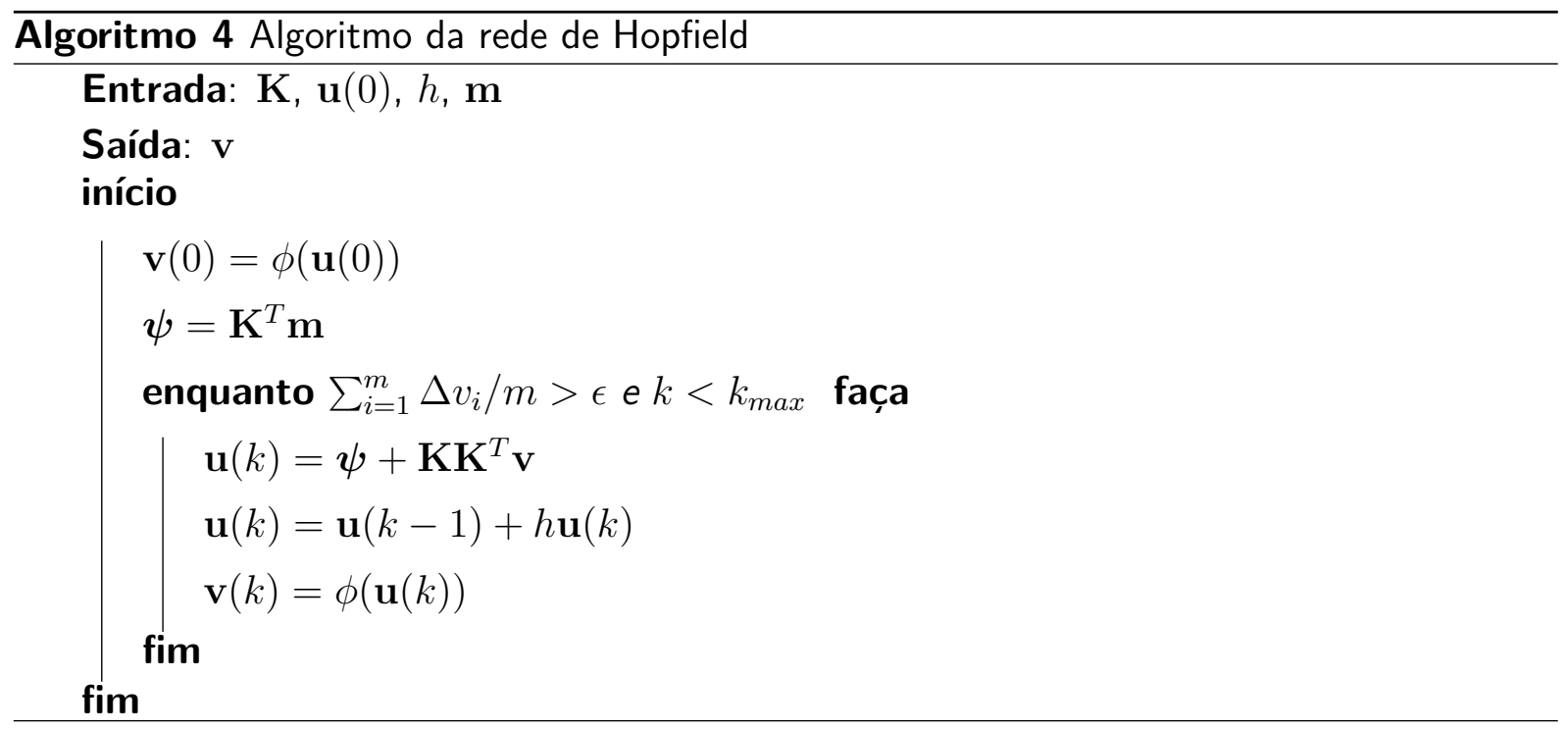

\subsection{Solução dos mínimos quadrados e decomposição em valores singulares (SVD) com regularização de Tikho- nov}

A decomposição em valores singulares junto ao algoritmo dos mínimos quadrados é a ferramenta mais utilizada na análise de problemas mal-postos e mal-condicionados e consequentemente das equações integrais de Fredholm do primeiro tipo, de modo que a comparação com os outros métodos propostos neste trabalho é certamente indispensável.

\subsubsection{SVD}

A decomposição em valores singulares é aplicável a qualquer matriz e é motivada pelo fato de que a imagem de uma esfera unitária $\mathbf{S} \in \Re^{n}$ sob a ação uma matriz qualquer $\mathbf{A} \in \Re^{m \times n}$ resulta em uma hiper-elipse, isto é, a generalização $m$-dimensional de uma elipse. (37) Uma hiper-elipse no $\Re^{m}$ é gerada a partir dos alongamentos $\sigma_{1}, \sigma_{2}, \ldots, \sigma_{m}$ da esfera unitária nas direções $\mathbf{u}_{1}, \mathbf{u}_{\mathbf{2}}, \ldots, \mathbf{u}_{\mathbf{m}}$, de modo que os vetores $\sigma_{1} \mathbf{u}_{1}, \sigma_{2} \mathbf{u}_{2}, \ldots, \sigma_{m} \mathbf{u}_{\mathbf{m}}$ consistem nos semi-eixos principais da hiper-elipse.

De maneira simplificada, a SVD fatora uma matriz $\mathbf{A} \in \Re^{m \times n}$ no produto de três matrizes:

$$
\mathbf{A}=\mathbf{U} \boldsymbol{\Sigma} \mathbf{V}^{T}
$$


Figura 2.22 - Representação geométrica da SVD para uma matriz $2 \times 2$.

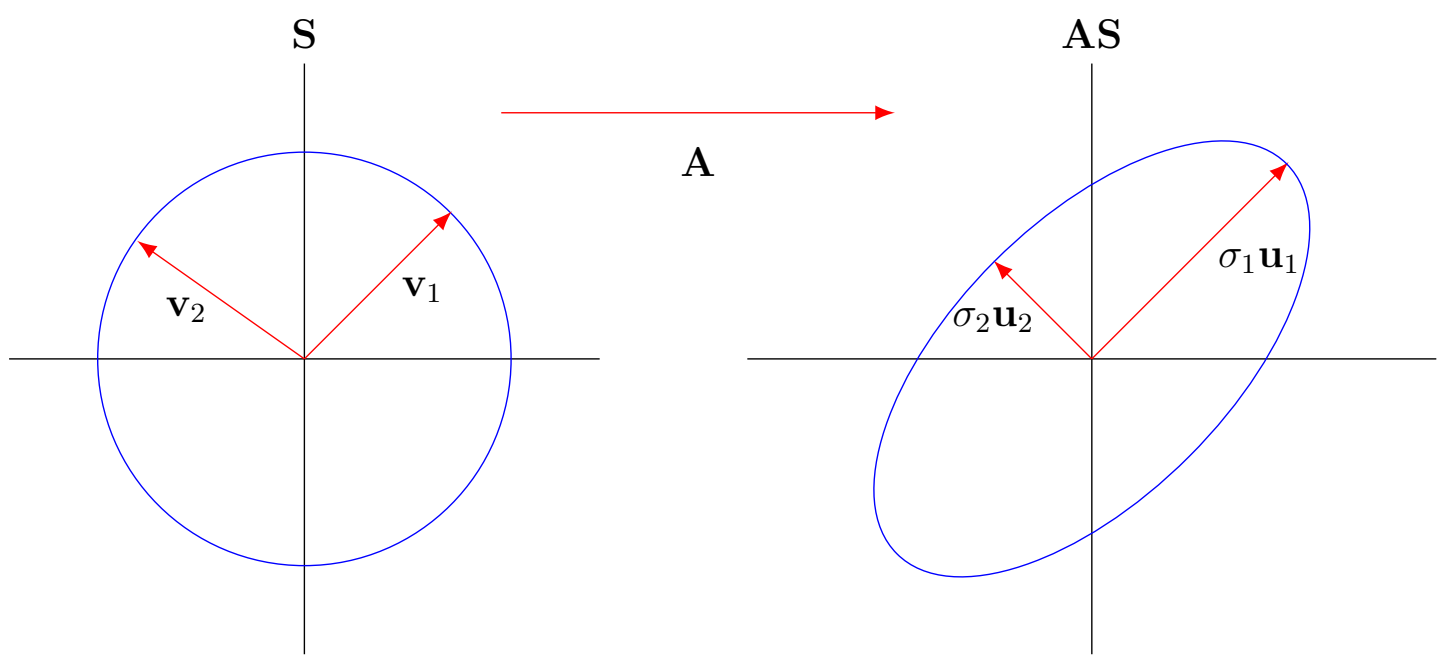

Fonte: Elaborada pelo autor.

onde $\mathbf{U} \in \Re^{m \times m}$ e $\mathbf{V} \in \Re^{n \times n}$ são ortonormais, de modo que

$$
\mathbf{U}^{T} \mathbf{U}=\mathbf{V}^{T} \mathbf{V}=\mathbf{I}
$$

e $\boldsymbol{\Sigma} \in \Re^{m \times n}$ é uma matriz diagonal cuja as entradas são todas positivas e dadas pela raízes quadradas dos autovalores de $\mathbf{A} \mathbf{A}^{T}$ ou $\mathbf{A}^{T} \mathbf{A}$. (38) Os $\sigma_{i}$ são os chamados valores singulares de $\mathbf{A}$ e normalmente estão ordenados de forma decrescente:

$$
\sigma_{1} \leq \sigma_{2} \leq \ldots \leq \sigma_{n} \leq 0
$$

Do exposto anteriormente, pode-se dizer então que:

- Os valores singulares de $\mathbf{A}$ podem ser interpretados como os comprimentos dos semieixos principais de AS.

- Os vetores coluna que compõem a matriz $\mathbf{U}$, chamados de vetores singulares à direita de $\mathbf{A}$ estão orientados nas direções dos semi-eixos principais de $\mathbf{A S}$ e os produtos $\sigma_{1} \mathbf{u}_{1}$, $\sigma_{2} \mathbf{u}_{2}, \ldots, \sigma_{m} \mathbf{u}_{m}$ consistem nestes semi-eixos principais.

- Os vetores singulares à esquerda de $\mathbf{A}$ são as contra-imagens dos semi-eixos principais de AS.

Além disso pode-se delinear a seguinte relação:

$$
\mathbf{A v}_{i}=\sigma_{i} \mathbf{u}_{i}
$$


Por fim, tendo em vista que $\mathbf{U}$ e $\mathbf{V}$ são ortonormais, a inversa de $\mathbf{A}$ é obtida de maneira simplificada,

$$
\mathbf{A}^{-1}=\mathbf{V} \mathbf{\Sigma}^{-1} \mathbf{U}^{T}
$$

Ressaltando que deve-se apenas tomar o devido cuidado para o aparecimento de singularidades em $\Sigma$.

\subsubsection{Algoritmo dos mínimos quadrados (LS - Least Squares}

Seja um sistema sobre-determinado definido por:

$$
\mathbf{A x}=\mathbf{b}, \quad \mathbf{A} \in \Re^{m \times n}, \quad m>n
$$

o vetor solução $\mathbf{x}$ pode ser obtido através do seguinte processo de minimização:

$$
\min _{\mathbf{x}}\left\{\frac{1}{2}\|\mathbf{A x}-\mathbf{b}\|^{2}\right\}
$$

onde,

$$
\|\mathbf{A x}-\mathbf{b}\|^{2}=\chi^{2}
$$

Portanto, a solução do problema se resume na minimização do erro quadrático $\chi^{2}$. A abordagem mais comum na solução de 2.8 .8 é tomar a derivada parcial de $\chi^{2}$ em relação ao vetor $\mathrm{x}$ e igualar a expressão à zero, isto é,

$$
\begin{gathered}
\chi^{2}=\mathbf{A}^{T} \mathbf{x} \mathbf{x}^{T} \mathbf{A}+\mathbf{b}^{T} \mathbf{b}-2 \mathbf{A} \mathbf{x}^{T} \mathbf{b} \\
\frac{\partial \chi^{2}}{\partial \mathbf{x}}=0 \\
\mathbf{x}=\left(\mathbf{A}^{T} \mathbf{A}\right)^{-1} \mathbf{A}^{T} \mathbf{b}
\end{gathered}
$$

onde o termo $\left(\mathbf{A}^{T} \mathbf{A}\right)^{-1} \mathbf{A}^{T}$ é conhecido como pseudo-inversa de Moore-Penrose e é normalmente representado por $\mathbf{A}^{+}$. 


\subsubsection{Determinação das distribuições de tempo de relaxação}

Procedendo como nos capítulos anteriores considera-se novamente as distribuições de valores $T_{1}$ ou $T_{2}$ simuladas por meio de distribuições multi log-normal dadas por 2.6.39 e os respectivos sinais de magnetização simulados $M\left(\tau_{j}^{*}\right)$ descritos por 2.6.42, deste modo pode-se definir:

$$
\mathbf{A} \triangleq \mathbf{K}=\left[\begin{array}{ccc}
K\left(\tau_{1}^{*}, \Gamma_{1}\right) & \cdots & K\left(\tau_{1}^{*}, \Gamma_{n}\right) \\
\vdots & \ddots & \vdots \\
K\left(\tau_{m}^{*}, \Gamma_{1}\right) & \cdots & K\left(\tau_{m}^{*}, \Gamma_{n}\right)
\end{array}\right]
$$

isto é, em 2.8.8, a matriz A corresponde aos os núcleos da equação de Fredholm que descreve o experimento ordenados na forma de uma matriz definida por $\mathbf{K}$.

O sinal de magnetização medido $M\left(\tau_{j}^{*}\right)$ escrito na forma de um vetor $\mathbf{m}$ corresponde a b em 2.8.8 e finalmente a distribuição de tempos de relaxação procurada $\mathrm{g}$ corresponde ao vetor solução $\mathrm{x}$, deste modo pode-se reescrever 2.8 .7 como:

$$
\min _{\mathbf{g}}\left\{\frac{1}{2}\|\mathbf{K g}-\mathbf{m}\|^{2}\right\}
$$

Contudo, lembrando que este problema não tem solução exata e pertence a classe dos problemas mal-postos é necessário impor restrições em 2.8.13 de modo a regularizar a solução desejada. Com o intuito de evitar detalhes excessivos na solução, utiliza-se o mesmo método de regularização descrito no algoritmo LMS-RNN, isto é, o método de regularização de Tikhonov. Assim, o problema de minimização toma a seguinte forma:

$$
\min _{\mathbf{g}}\left\{\frac{1}{2}\|\mathbf{K g}-\mathbf{m}\|^{2}+\frac{1}{2} \lambda\|\mathbf{L g}\|^{2}\right\}
$$

consequentemente 2.8.9 agora é dada por:

$$
\chi^{2}=\mathbf{A}^{T} \mathbf{x} \mathbf{x}^{T} \mathbf{A}+\mathbf{b}^{T} \mathbf{b}-2 \mathbf{A} \mathbf{x}^{T} \mathbf{b}+\mathbf{L}^{T} \mathbf{w} \mathbf{w}^{T} \mathbf{L}
$$

onde $\mathbf{L}$ é o operador diferencial de segunda ordem aproximado descrito em 2.6.60 e $\lambda$ é o parâmetro que controla a regularização da solução, neste caso, controla a suavidade da resposta. A determinação de um $\lambda$ ideal é um problema à parte e embora não exista um método bem definido existem diversas abordagens bem sucedidas, como por exemplo o método da curva $L$ descrito em (39)(40), o método da validação cruzada demonstrado em (41), além do método da parcimônia. (42)

A solução do problema 2.8.14 é obtida tomando a derivada parcial de $\chi^{2}$ em relação a $\mathbf{g}$ 
Figura 2.23 - Suavização da resposta (regularização) para diferentes valores de $\lambda$.

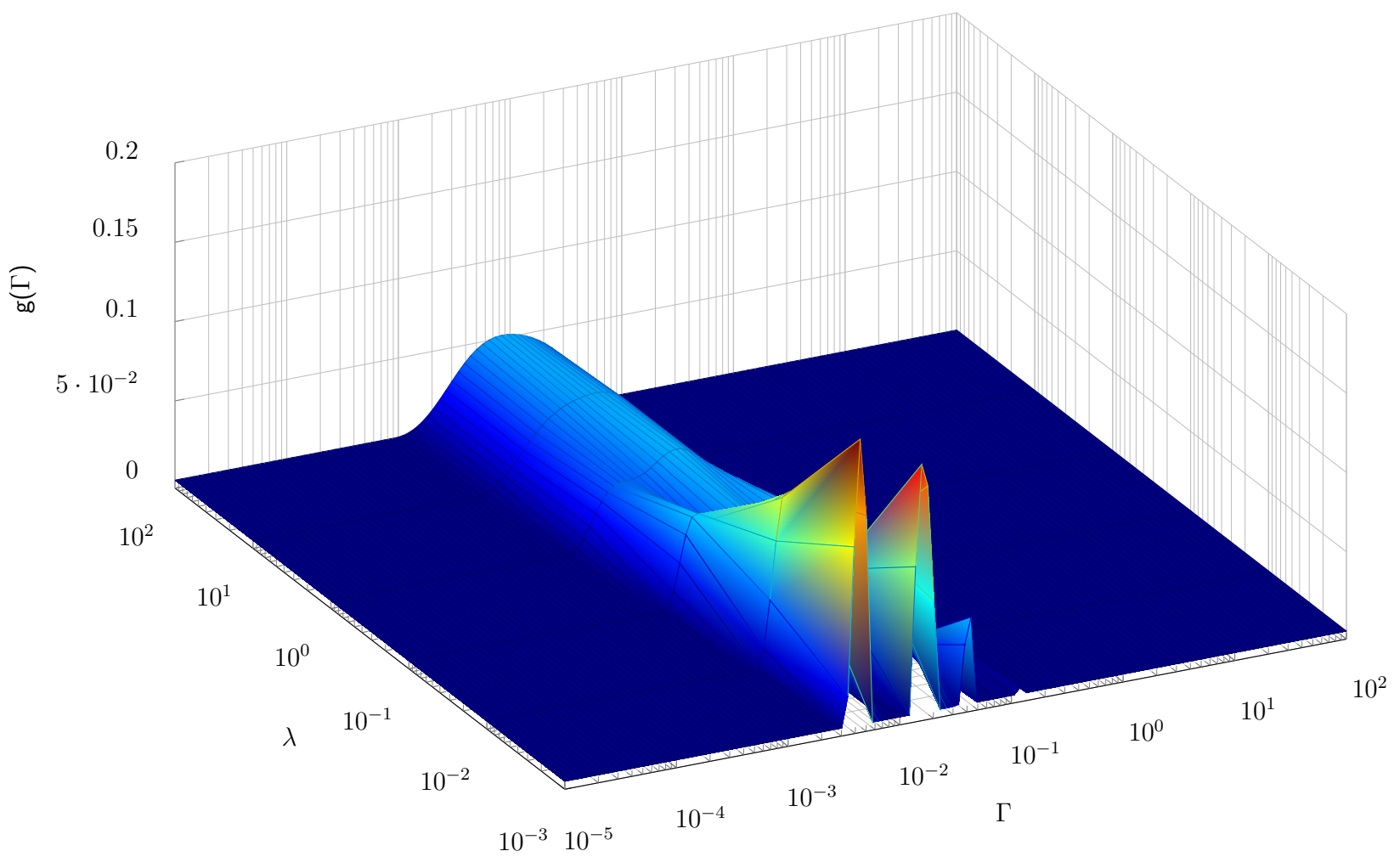

Fonte: Elaborada pelo autor.

e igualando a expressão a zero,

$$
\begin{gathered}
\frac{\partial \chi^{2}}{\partial \mathbf{g}}=-\mathbf{K}^{T} \mathbf{m}+\mathbf{K}^{T} \mathbf{K g}+\lambda \mathbf{L}^{T} \mathbf{L g}=0 \\
\left(\mathbf{K}^{T} \mathbf{K}+\lambda \mathbf{L}^{T} \mathbf{L}\right) \mathbf{g}=\mathbf{K}^{T} \mathbf{m}
\end{gathered}
$$

a solução dos mínimos quadrados é dada então por:

$$
\mathbf{g}=\left(\mathbf{K}^{T} \mathbf{K}+\lambda \mathbf{L}^{T} \mathbf{L}\right)^{-1} \mathbf{K}^{T} \mathbf{m}
$$

Entretanto, a matriz $\left(\mathbf{K}^{T} \mathbf{K}+\lambda \mathbf{L}^{T} \mathbf{L}\right)$ é singular e sua inversa é calculada através da decomposição em valores singulares. Por fim, a distribuição dos tempos de relaxação é obtida através da seguinte expressão:

$$
\mathbf{g}=\mathbf{V} \boldsymbol{\Sigma}^{-1} \mathbf{U}^{T} \mathbf{K}^{T} \mathbf{m}
$$

Deve-se ressaltar ainda que a solução 2.8 .19 pode possuir componentes negativas, de modo que é necessário impor não-negatividade da solução. A imposição é realizada através de um processo iterativo que consiste em analisar a solução 2.8.19 em busca das componentes $g_{i}<0$. Para cada $g_{i}$ negativo encontrado soma-se um valor positivo elevado na componente 
$(i, i)$ da matriz $\mathbf{L}^{T} \mathbf{L}$ e em seguida repete-se o processo até que a soma dos quadrados dos $g_{i}<0$ alcance um limite pré-estabelecido. Ao fim do processo de imposição de negatividade algumas componentes de $\mathrm{g}$ ainda possuirão valores negativos, mas pouco significativos. 


\section{Técnica Proposta, Testes e Resultados}

Neste trabalho, a comparação entre os algoritmos está em princípio direcionada a demonstrar a existência de meios alternativos na solução do problema exposto. O segundo ponto é comparar a qualidade das respostas entre os algoritmos, considerando o erro médio quadrático e a não-negatividade das soluções. Por fim analisa-se também o tempo necessário para convergência de cada algoritmo.

Tendo em vista a dificuldade de se obter meios porosos com distribuições de tamanhos de poros bem determinadas a principal forma de avaliação dos métodos propostos neste trabalho se dá através de dados simulados. Além destas simulações, há também verificações com sinais de magnetização obtidos em experimentos reais envolvendo análise de solos. Estes dados foram cedidos pelo Prof. Dr. Tito José Bonagamba (Grupo de Ressonância Magnética Nuclear Institudo de Física de São Carlos).

Os dados simulados foram obtidos conforme descrito nas sessões anteriores através de um gerador de distribuição desenvolvido no matlab.

Com exceção da decomposição em valores singulares, cujo algoritmo é relativamente complexo e de modo a garantir o bom desempenho optou-se por utilizar uma versão (43) bastante conhecida e já otimizada, todos os demais algoritmos foram integralmente implementados pelo autor em linguagem padrão $\mathrm{C} / \mathrm{C}++$. Os trechos de código favoráveis ao paralelismo foram paralelizadas através de uma biblioteca de multiprocessamento chamada OpenMP. (44)

\subsection{Metodologias}

Os dados simulados foram gerados de modo que a soma das amplitudes máximas das distribuições fosse sempre unitária e antes de serem processados nos algoritmos foram ainda normalizados através do seguinte procedimento:

$$
M\left(\tau_{j}^{*}\right)=\frac{M\left(\tau_{j}^{*}\right)}{\sum_{j=1}^{m} M\left(\tau_{j}^{*}\right)}, \quad \text { para } \mathrm{j}=1,2, \ldots, \mathrm{m} .
$$


Figura 3.1 - Interface do gerador de pares de sinais simulados desenvolvido.

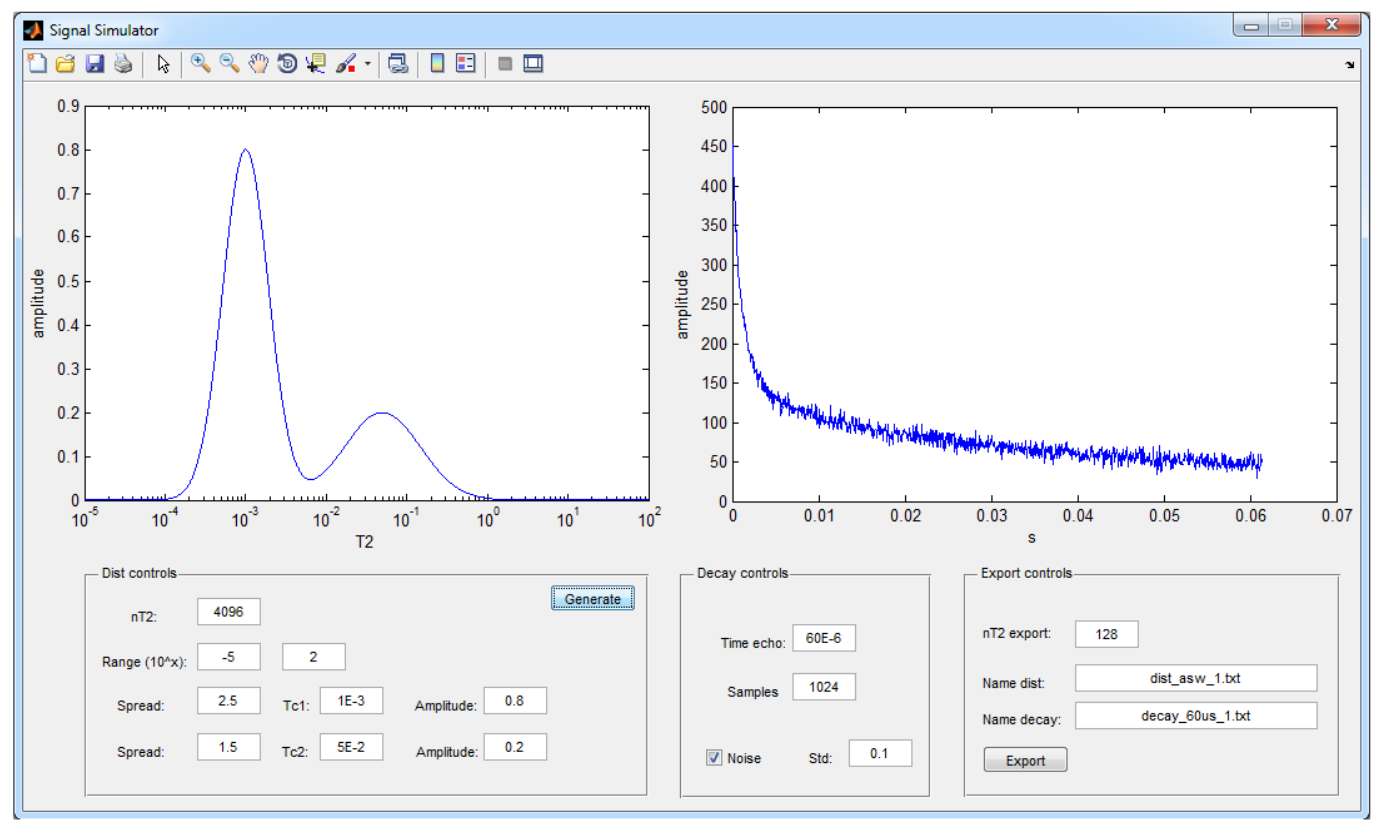

Fonte: Elaborada pelo autor.

Isto é, a soma das componentes do sinal é também unitária. Este processo é fundamental no caso das redes recorrentes de Hopfield tendo em vista os limites de saturação da nãolinearidade (função logística) que compõe os neurônios artificiais.

\subsubsection{LMS-NN e LMS-RNN}

Após o desenvolvimento e implementação de cada abordagem do algoritmo LMS, para cada sinal analisado diversas combinações de valores para os parâmetros taxa de convergência $\eta$ e regularização $\lambda$ foram testados em busca de convergência suave e que apresentasse resposta aceitável.

Além dos parâmetros de convergência e de regularização avaliou-se também as diferentes respostas das abordagens do algoritmo frente a distribuições simuladas idênticas, entretanto com sinais de multi-decaimento exponencial considerando diferentes tempos de eco.

Avaliou-se também o comportamento do algoritmo para diferentes valores iniciais $\mathbf{g}_{0} \mathrm{e}$ por fim explorou-se a principal característica do LMS, a possibilidade de considerar diferentes fontes de sinal. 


\subsubsection{Redes recorrentes de Hopfield}

As redes recorrentes de Hopfield tem uma característica de convergência bastante dependente dos valores iniciais (relacionada com as chamadas bacias de atração), deste modo, em principio, avaliou-se diversas possibilidades de valores para os parâmetros iniciais da rede.

Assim como realizado para o algoritmo LMS avaliou-se também as diferentes respostas do algoritmo frente a distribuições simuladas idênticas e com sinais de multi-decaimento exponencial considerando diferentes tempos de eco.

\subsubsection{Solução dos mínimos quadrados com decomposição em valores singulares e regularização de Tikhonov}

O ajuste do parâmetro de regularização no algoritmo dos mínimos quadrados regularizado foi baseado no método da parcimônia, isto é, avaliou-se para cada sinal uma faixa de valores para o parâmetro de regularização $\lambda$ entre $10^{-5}$ e $10^{5}$ em passos de potência de dez, definindose como o valor ideal aquele $\lambda$ que dentre os melhores resultados produz o maior erro quadrático médio.

De modo a comparar com as abordagens do LMS e da rede recorrente de Hopfield, a mesma avaliação envolvendo distribuições simuladas idênticas e com sinais de multi-decaimento exponencial diferentes foi realizada para o método dos mínimos quadrados.

Para o processo de imposição de não-negatividade foi definido como valor de parada $E_{-}=10^{-8}$, onde $E_{-}$é a energia (em processamento digital de sinais a energia de um sinal é definida como a soma dos quadrados das componentes do sinal) das componentes negativas de g. Além disso, o processo foi interrompido quando constado instabilidade ou quando o número de iterações ultrapassasse $k_{\max }=1000$.

\subsection{Teste 1 - distribuições simuladas próximas}

O primeiro teste foi realizado a partir da simulação de uma distribuição de valores $T_{2}$ obtida através de uma distribuição multi log-normal formada por $2^{16}$ pontos logaritmicamente espaçados no intervalo de $10^{-4} s$ à $10^{1} s$ e contendo dois picos relativamente próximos cen- 
trados em $10^{-2}$ e $10^{-1}$. A partir desta distribuição foram obtidos 6 diferentes simulações de sinais de magnetização contaminados com ruído branco e contendo $2^{12}$ pontos cada. Estes foram realizados variando o valor do tempo de eco $\tau$ partindo de $15 \mu \mathrm{s}$ e multiplicando este valor a cada teste por uma potência de dois, isto é $\tau_{i}=15\left(2^{i}\right) \mu s$ para $i=0,1, \ldots, 5$. Este procedimento foi realizado para verificar a influência do núcleo da equação de Fredholm no andamento da inversão, na qualidade dos resultados e as vantagens da possibilidade de combinar vários sinais em uma única análise.

\subsubsection{LMS-NN}

Seguem abaixo os gráficos das distribuições alcançadas por meio do algoritmo dos mínimos quadrados médios com restrição de não negatividade para cada um dos seis sinais de decaimento simulados conforme descrito acima. Os parâmetros utilizados em cada um dos sinais, além do erro médio quadrático em relação a distribuição simulada estão relacionados na tabela 3.1 .

Figura 3.2 - Distribuição obtida através do algoritmo LMS-NN para o sinal simulado com $\tau^{*}=30 \mu \mathrm{s}$ e comparado à distribuição simulada do teste um.

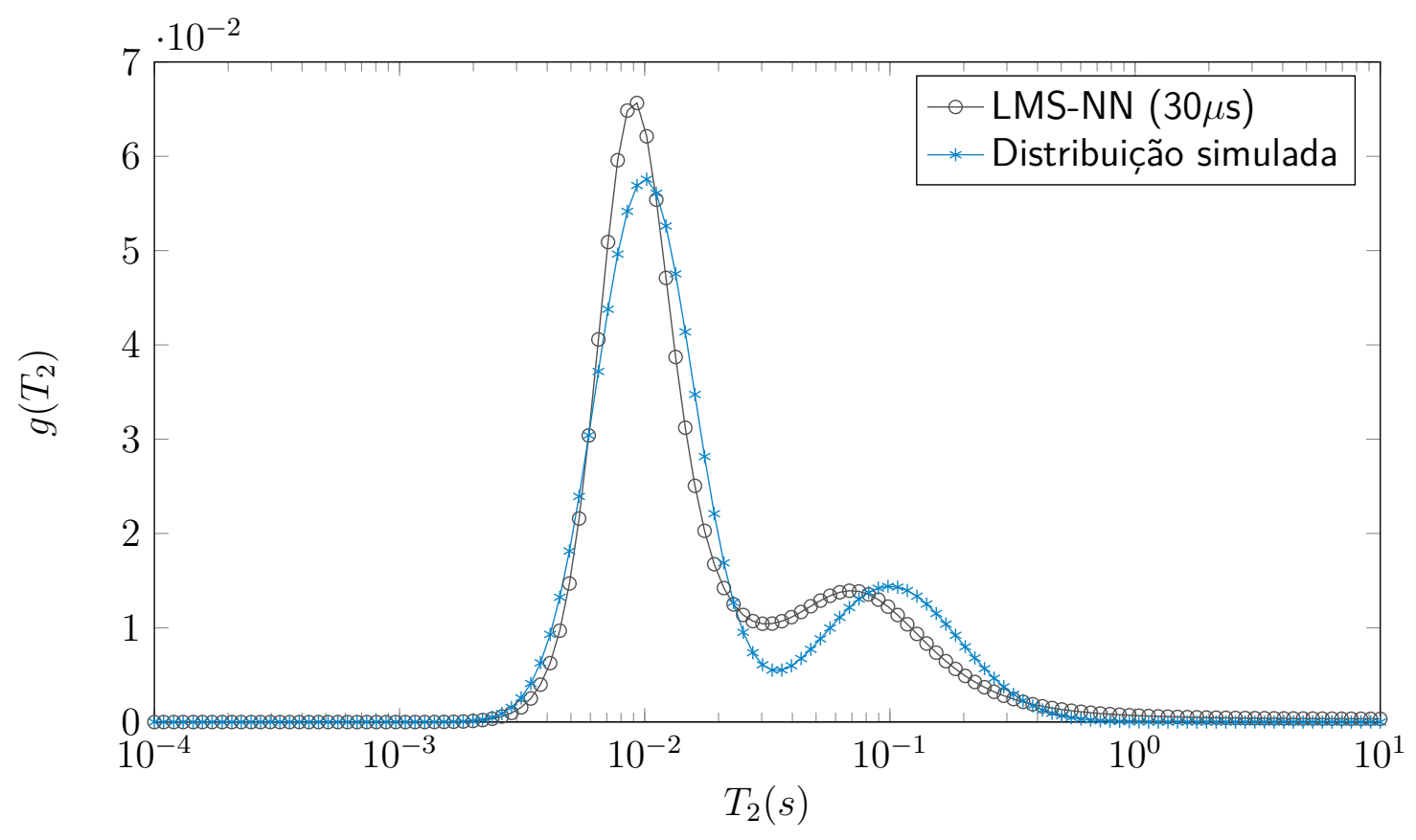

Fonte: Elaborada pelo autor. 
Figura 3.3 - Distribuição obtida através do algoritmo LMS-NN para o sinal simulado com $\tau^{*}=60 \mu \mathrm{s}$ e comparado à distribuição simulada do teste um.

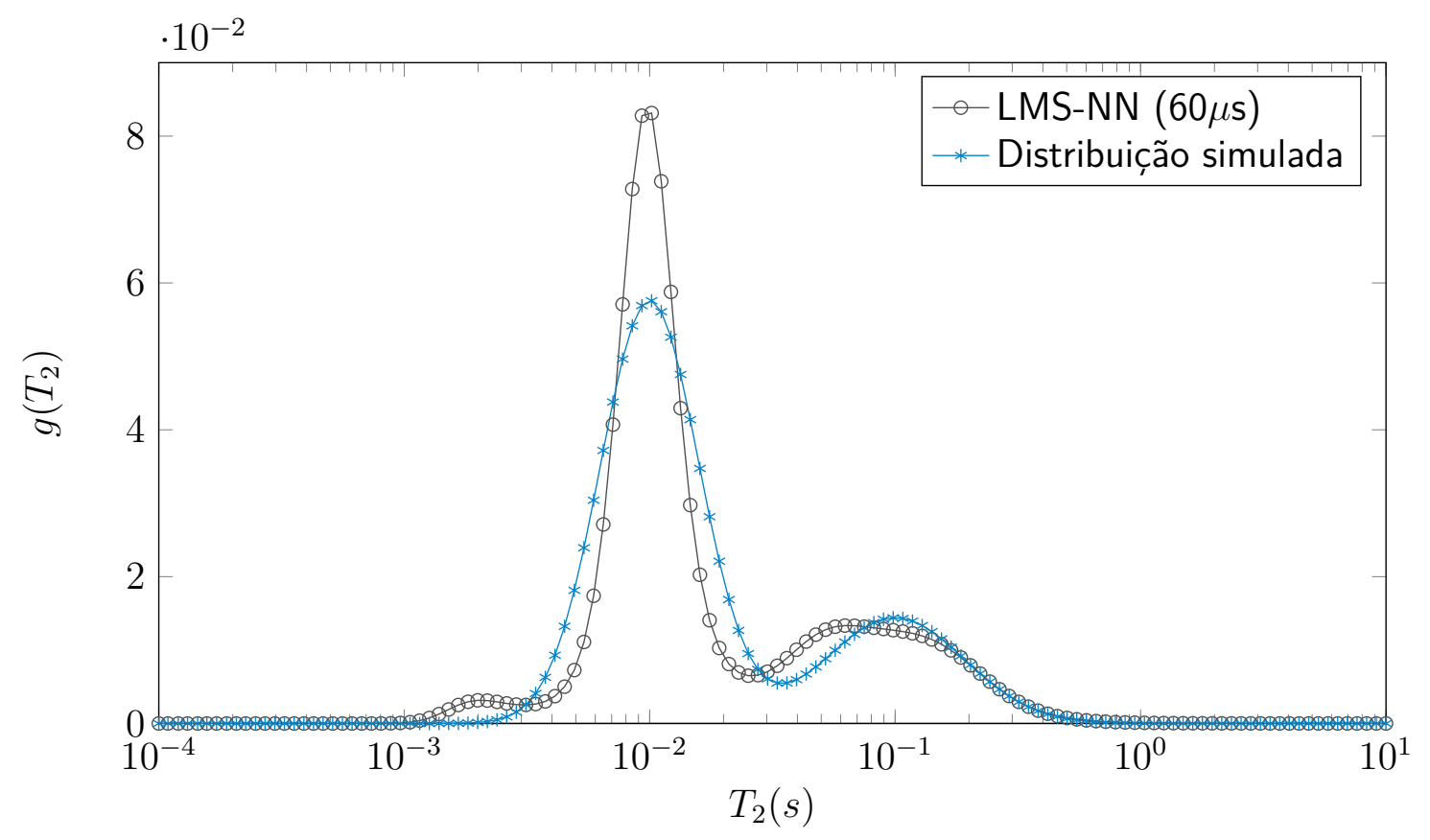

Fonte: Elaborada pelo autor.

Figura 3.4 - Distribuição obtida através do algoritmo LMS-NN para o sinal simulado com $\tau^{*}=$ $120 \mu \mathrm{s}$ e comparado à distribuição simulada do teste um.

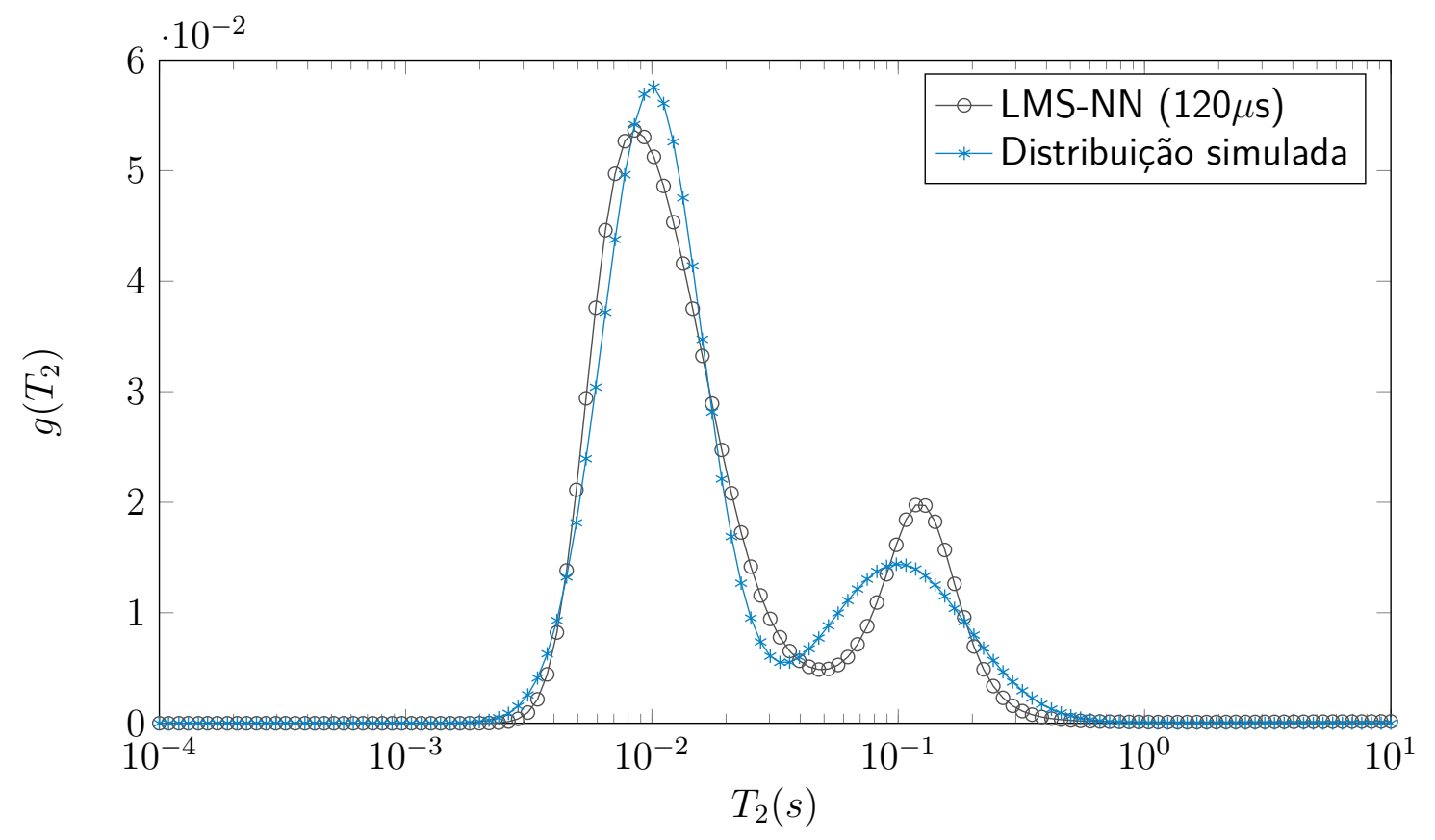

Fonte: Elaborada pelo autor. 
Figura 3.5 - Distribuição obtida através do algoritmo LMS-NN para o sinal simulado com $\tau^{*}=$ $240 \mu s$ e comparado à distribuição simulada do teste um.

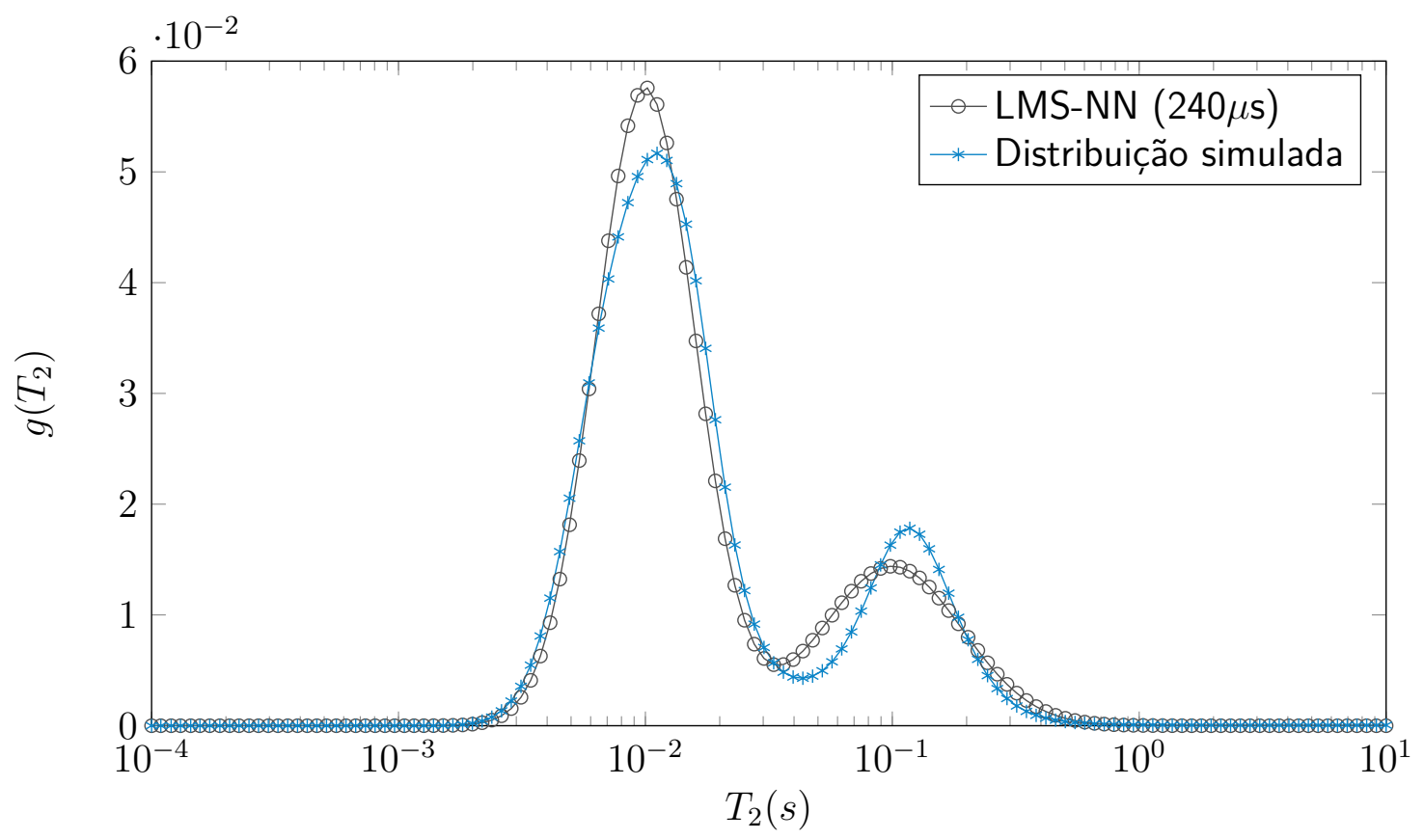

Fonte: Elaborada pelo autor.

Figura 3.6 - Distribuição obtida através do algoritmo LMS-NN para o sinal simulado $\operatorname{com} \tau^{*}=$ $480 \mu \mathrm{s}$ e comparado à distribuição simulada do teste um.

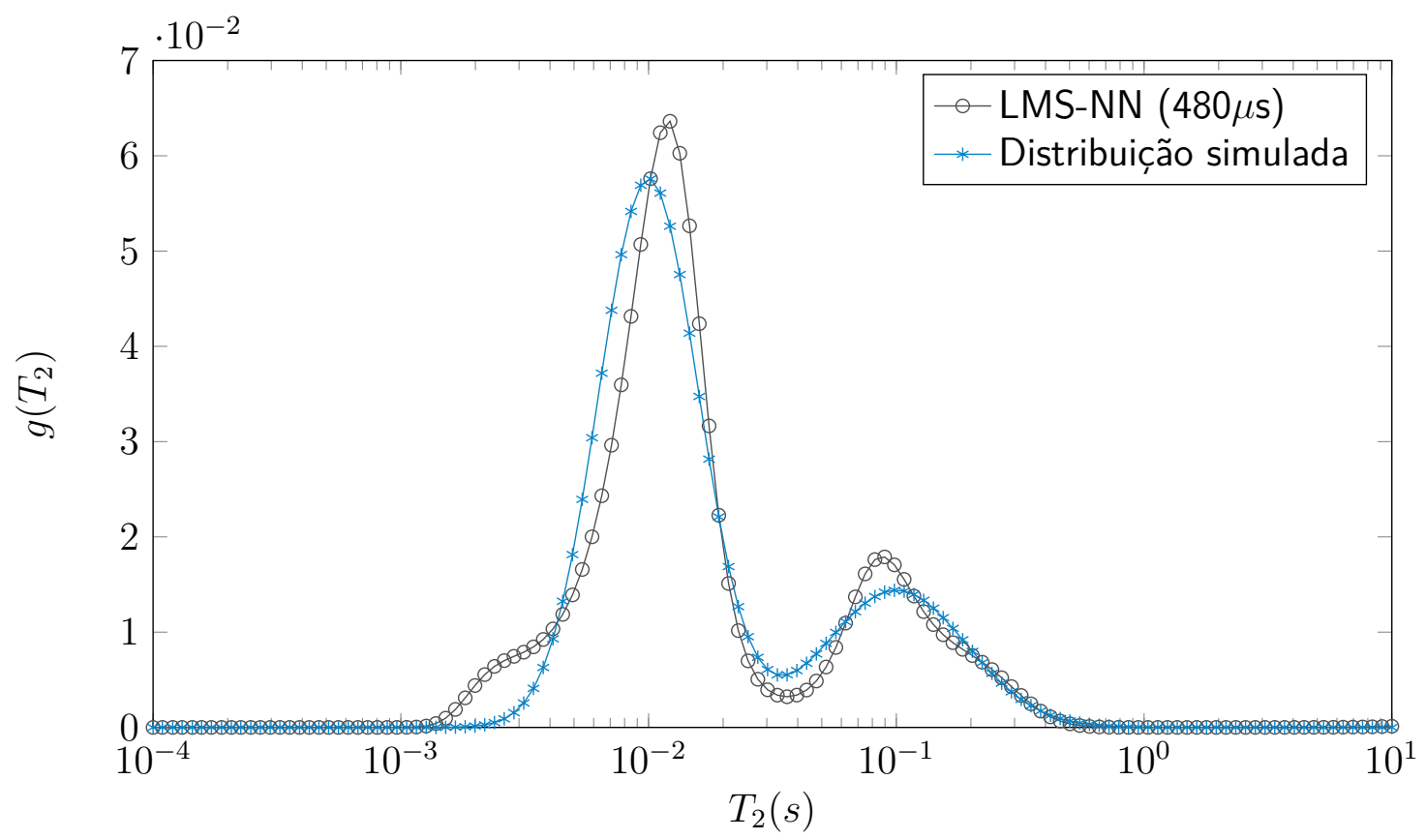

Fonte: Elaborada pelo autor. 
Figura 3.7 - Distribuição obtida através do algoritmo LMS-NN para o sinal simulado $\operatorname{com} \tau^{*}=$ $960 \mu \mathrm{s}$ e comparado à distribuição simulada do teste um.

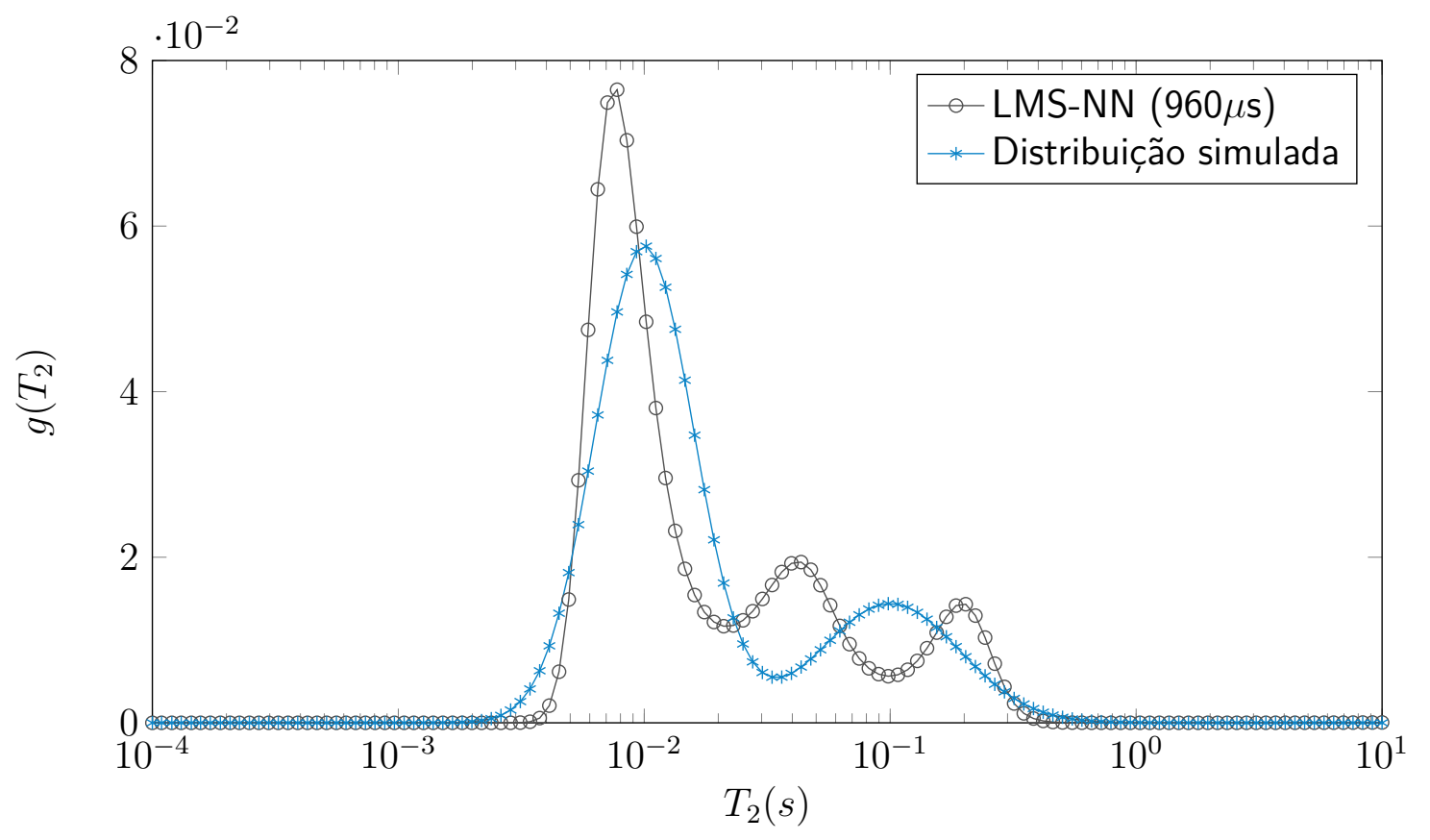

Fonte: Elaborada pelo autor. 
Figura 3.8 - Distribuição obtida através do algoritmo LMS-NN combinando os sinais simulados com $\tau^{*}=30 \mu s, 60 \mu s, 120 \mu s, 240 \mu s, 480 \mu s$ e $960 \mu s$ e comparado à distribuição simulada para o teste um.

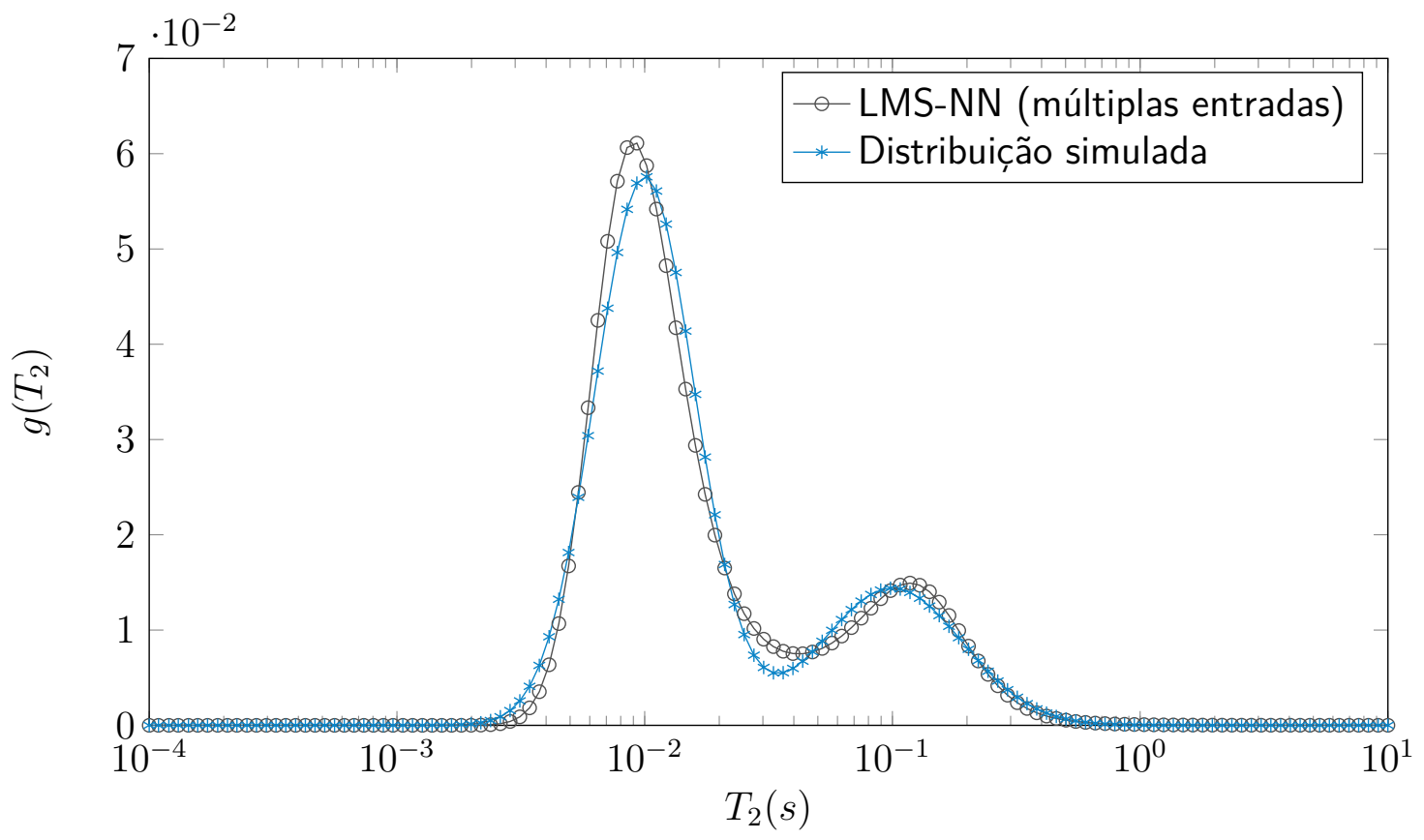

Fonte: Elaborada pelo autor.

Tabela 3.1 - Parâmetros utilizados e resultados obtidos com algoritmos LMS-NN.

\begin{tabular}{cccccc}
$\tau^{*}$ & $\eta$ & iterações & tempo(s) & convergência média & MSE \\
\hline \hline $30 \mu s$ & $10^{-5}$ & 6516 & 250 & $5.10^{-4}$ & $9,06.10^{-6}$ \\
\hline $60 \mu s$ & $10^{-5}$ & 20269 & 783 & $5.10^{-4}$ & $2,93.10^{-5}$ \\
\hline $120 \mu s$ & $10^{-5}$ & 8921 & 428 & $5.10^{-4}$ & $6,44.10^{-5}$ \\
\hline $240 \mu s$ & $10^{-5}$ & 15558 & 580 & $5.10^{-4}$ & $4,39.10^{-5}$ \\
\hline $480 \mu s$ & $10^{-5}$ & 38208 & 1917 & $5.10^{-4}$ & $1,38.10^{-5}$ \\
\hline $960 \mu s$ & $10^{-5}$ & 18840 & 727 & $5.10^{-4}$ & $5,83.10^{-5}$ \\
\hline $30 \mu s, 60 \mu s, 120 \mu s$, & & & & & \\
$240 \mu s, 480 \mu s, 960 \mu s$ & $10^{-5}$ & 510 & 80 & $5.10^{-4}$ & $3,47.10^{-6}$ \\
\hline
\end{tabular}

Fonte: Elaborada pelo autor.

Analisando os resultados da tabela 3.1 junto das figuras 3.2, 3.3, 3.4, 3.5, 3.6 e 3.7 é possível perceber que a variação do tempo de eco tem influência significativa no desempenho do algoritmo. Conforme $\tau^{*}$ assume valores que resultam em supressão das componentes significativas do sinal de magnetização a resposta do algoritmo vai se tornando mais imprecisa em determinadas regiões da distribuição. Contudo, analisando o último resultado da tabela 
3.1 junto do gráfico 3.8 observa-se que compor a análise no LMS-NN atráves de sinais com diferentes tempos de eco para distribuições com picos individuais, ou mesmo múltiplos e relativamente próximos, certamente tem efeito positivo no resultado final, além de aparentemente acelerar a convergência do algoritmo.

\subsubsection{LMS-RNN}

Abaixo seguem os gráficos das distribuições alcançadas através do algoritmo dos mínimos quadrados médios com restrição de não negatividade e regularização de Tikhonov para cada um dos seis sinais de decaimento simulados conforme descrito no inicio da sessão. Os parâmetros utilizados na análise pelo LMS-RNN com cada um dos sinais, além do erro médio quadrático da resposta em relação a distribuição simulada estão relacionados na tabela 3.2.

Figura 3.9 - Distribuição obtida através do algoritmo LMS-RNN para o sinal simulado $\operatorname{com} \tau^{*}=$ $30 \mu s$ e comparado à distribuição simulada do teste um.

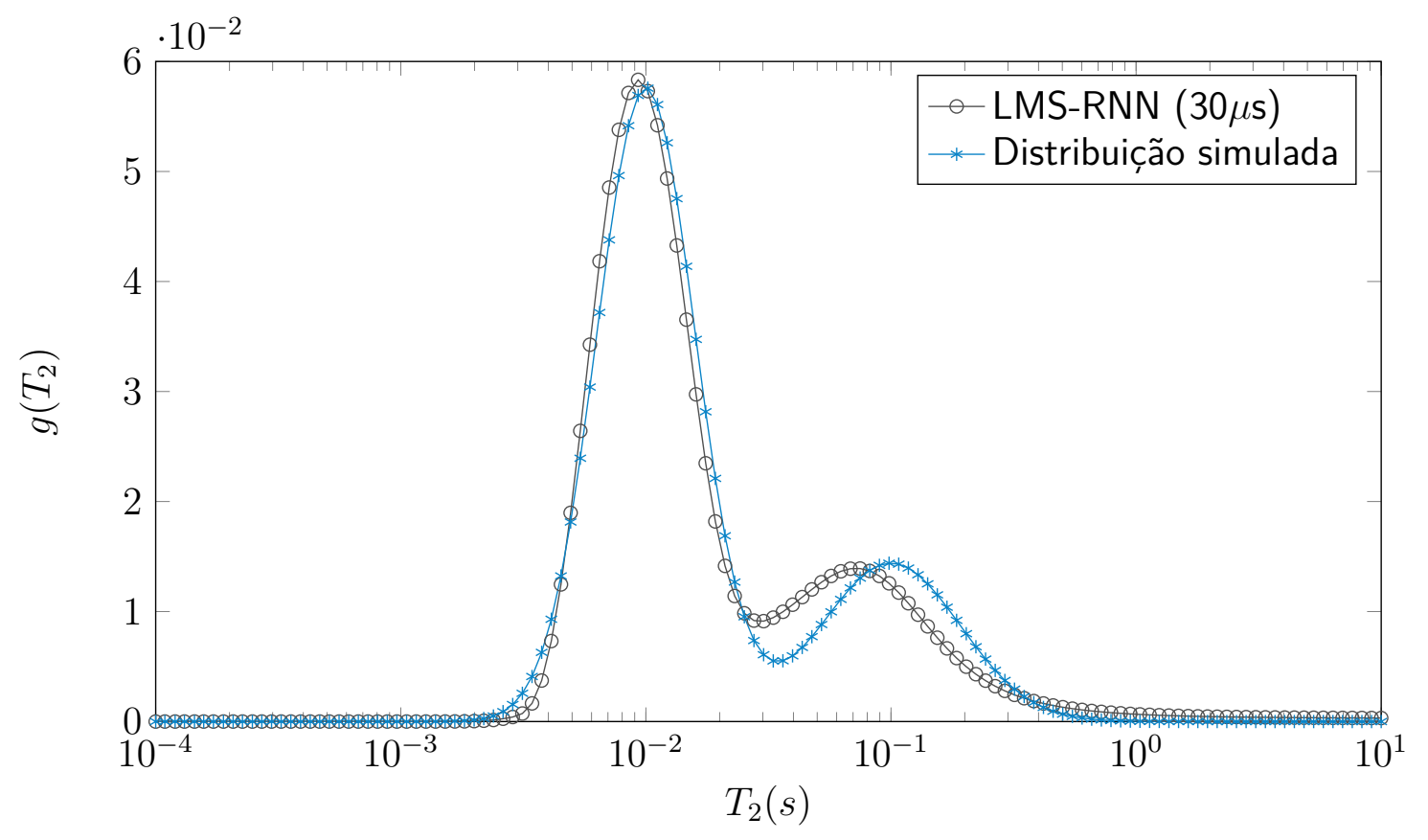

Fonte: Elaborada pelo autor. 
Figura 3.10 - Distribuição obtida através do algoritmo LMS-RNN para o sinal simulado $\operatorname{com} \tau^{*}=$ $60 \mu s$ e comparado à distribuição simulada do teste um.

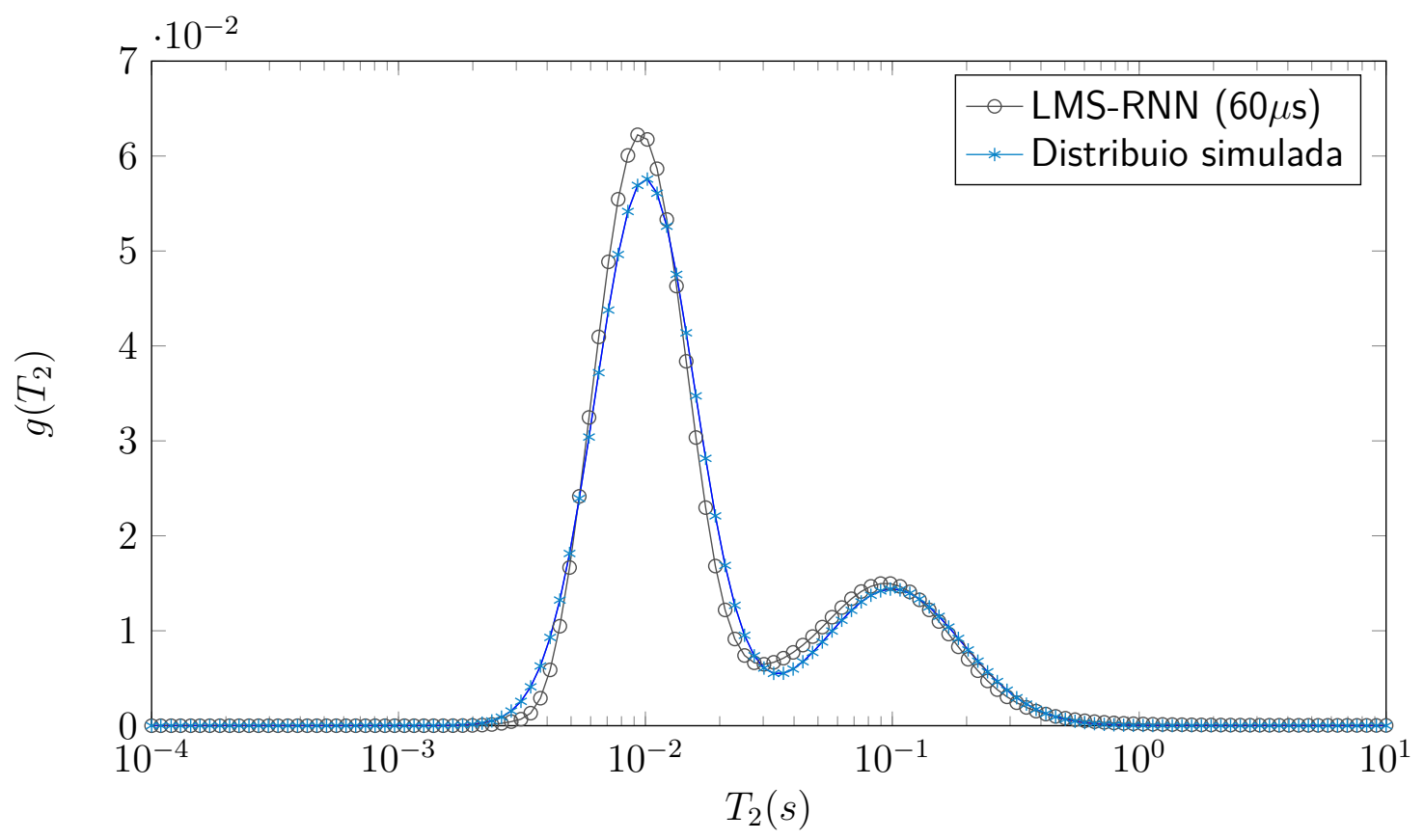

Fonte: Elaborada pelo autor.

Figura 3.11 - Distribuição obtida através do algoritmo LMS-RNN para o sinal simulado com $\tau^{*}=$ $120 \mu s$ e comparado à distribuição simulada do teste um.

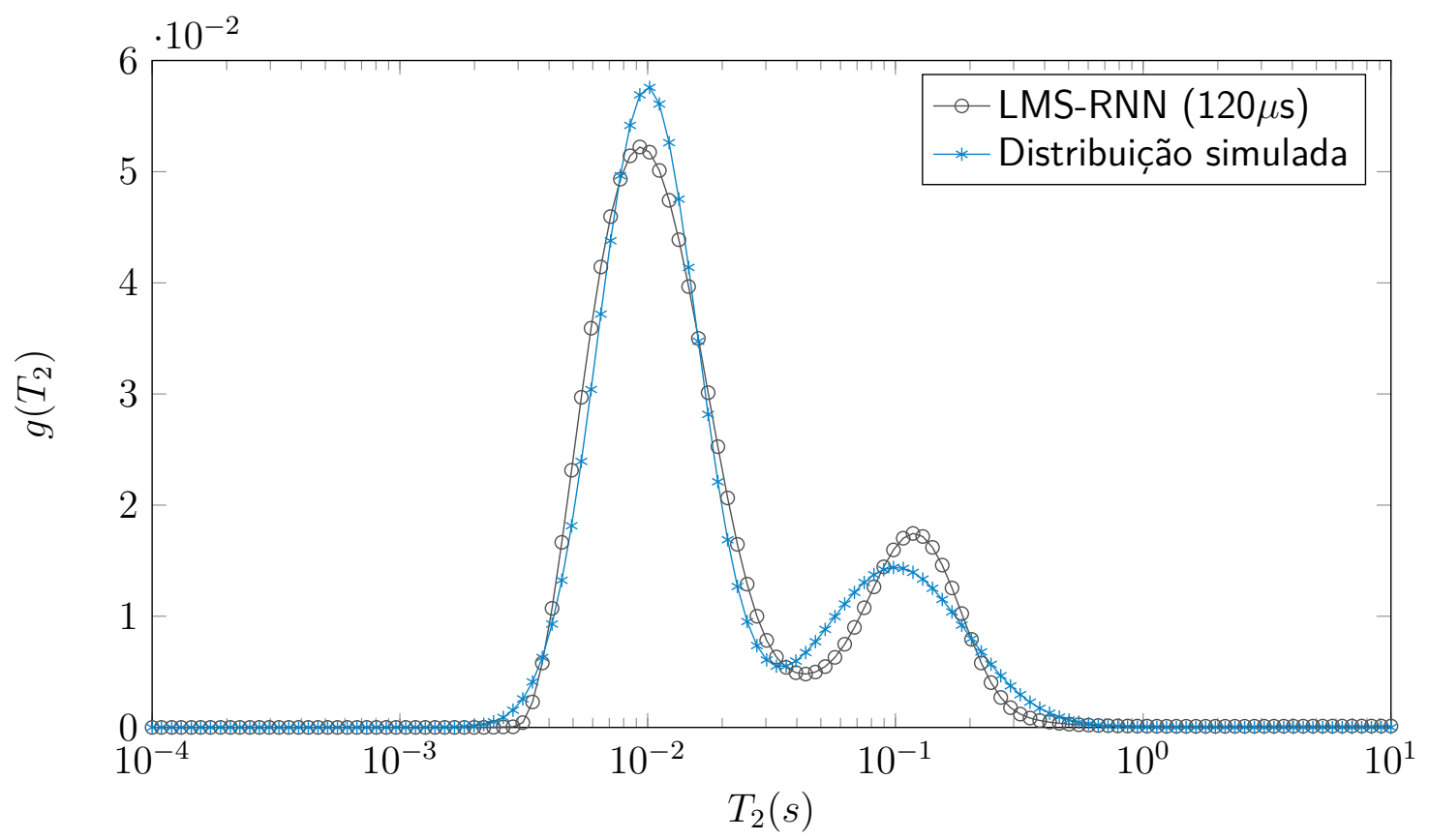

Fonte: Elaborada pelo autor. 
Figura 3.12 - Distribuição obtida através do algoritmo LMS-RNN para o sinal simulado com $\tau^{*}=$ $240 \mu \mathrm{s}$ e comparado à distribuição simulada do teste um.

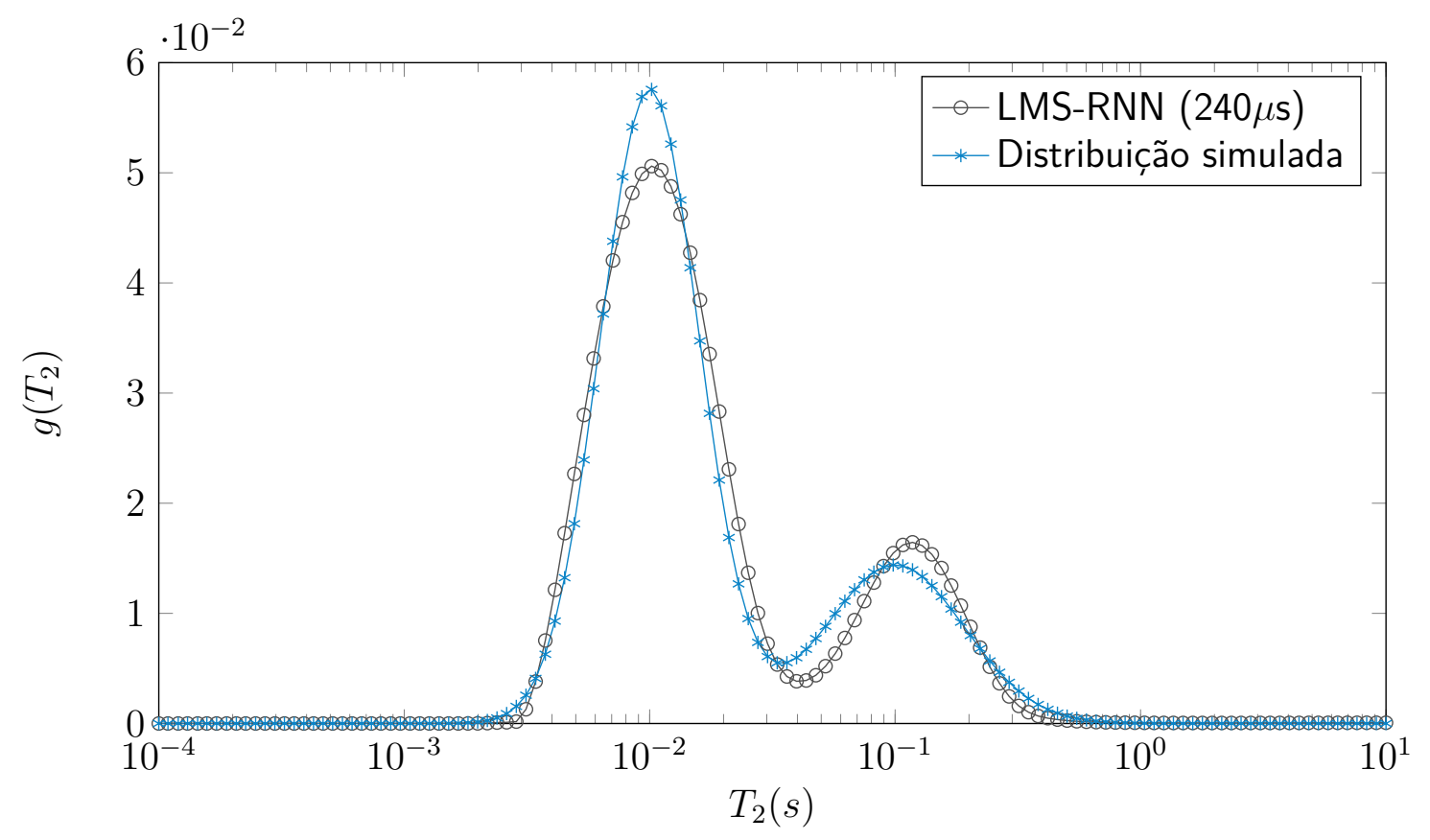

Fonte: Elaborada pelo autor.

Figura 3.13 - Distribuição obtida através do algoritmo LMS-RNN para o sinal simulado com $\tau^{*}=$ $480 \mu s$ e comparado à distribuição simulada do teste um.

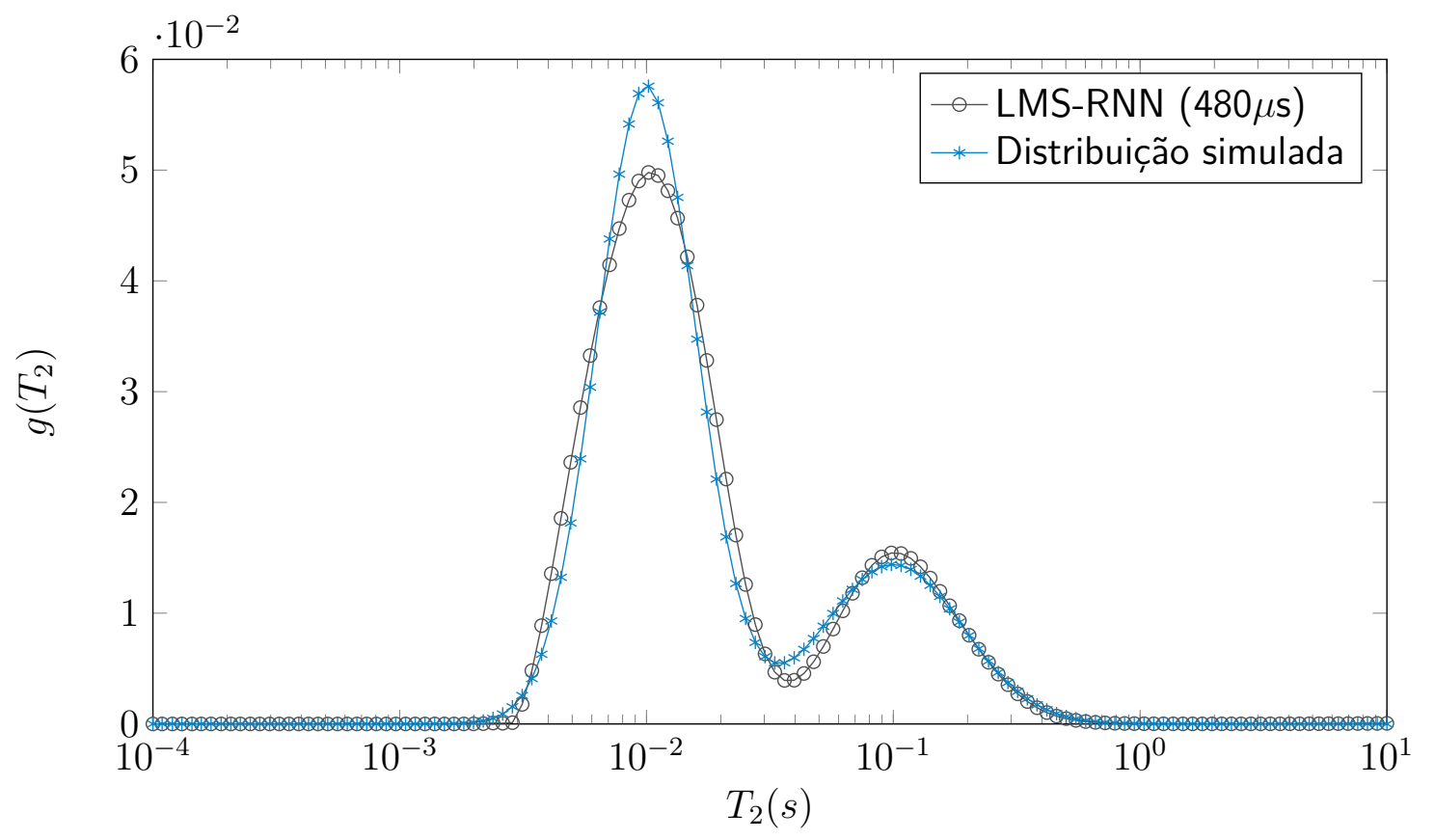

Fonte: Elaborada pelo autor. 
Figura 3.14 - Distribuição obtida através do algoritmo LMS-RNN para o sinal simulado com $\tau^{*}=$ $960 \mu s$ e comparado à distribuição simulada do teste um.

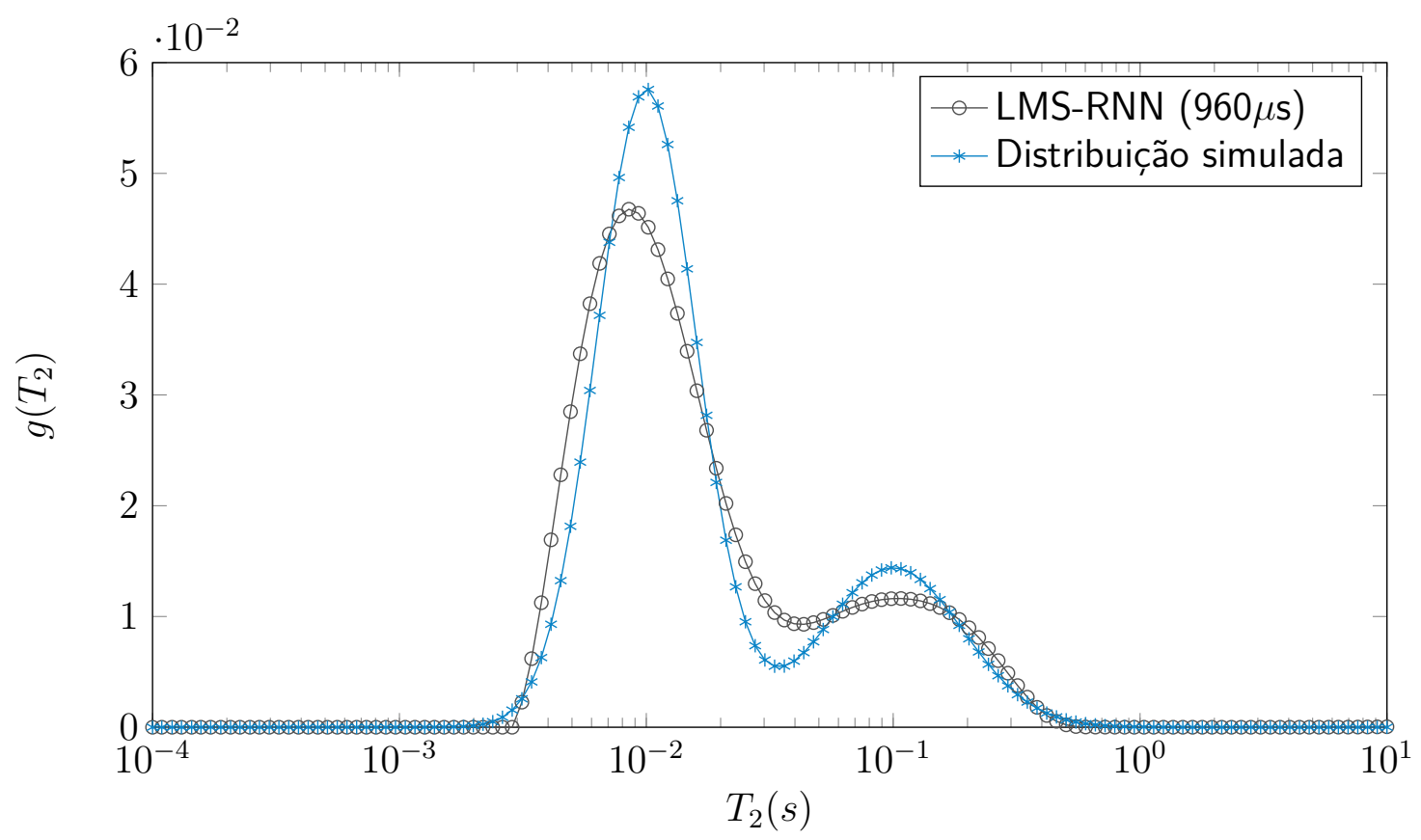

Fonte: Elaborada pelo autor. 
Figura 3.15 - Distribuição obtida através do algoritmo LMS-RNN combinando os sinais simulados com $\tau^{*}=30 \mu s, 60 \mu s, 120 \mu s, 240 \mu s, 480 \mu s$ e $960 \mu s$ e comparado à distribuição simulada para o teste um.

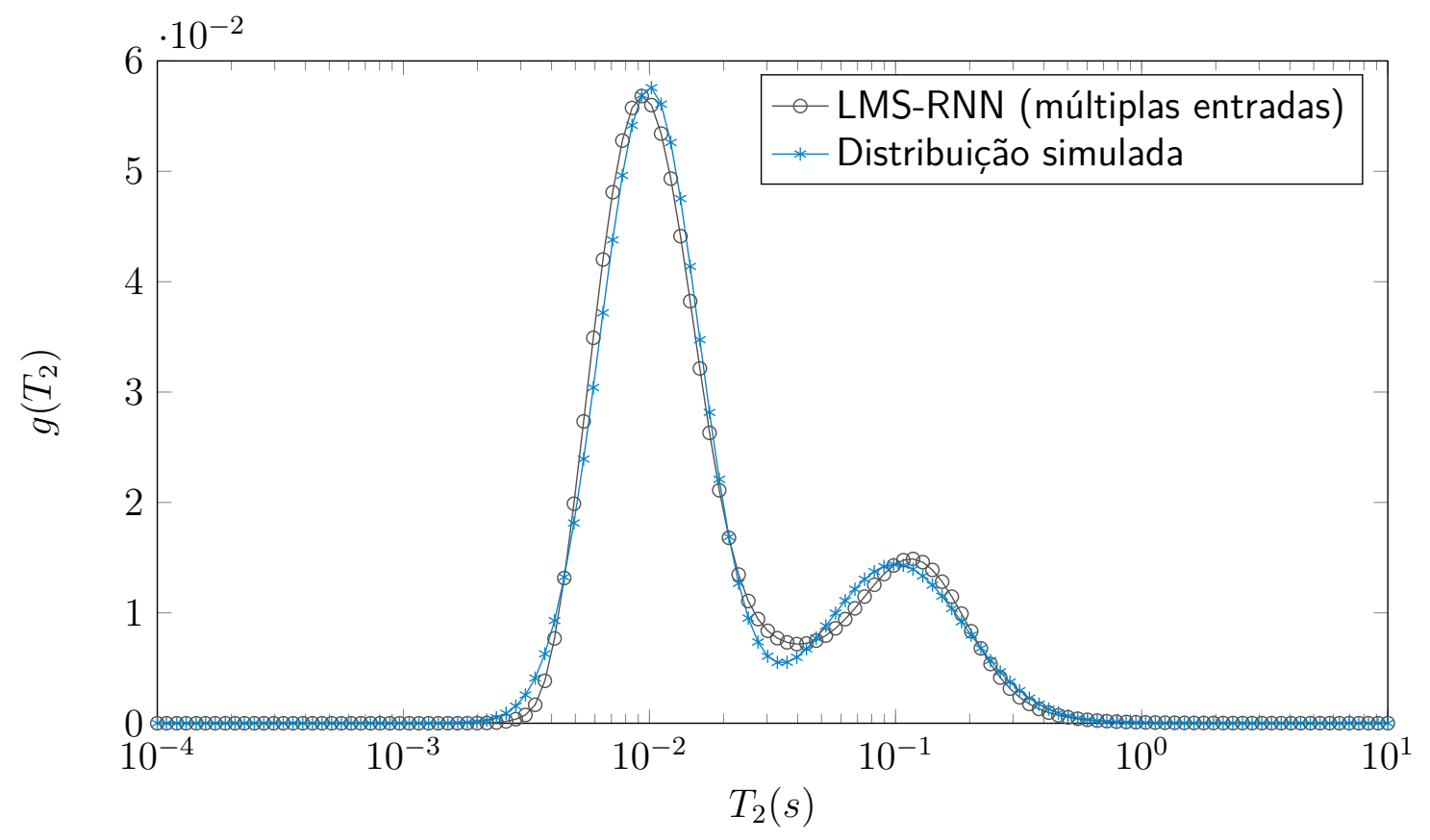

Fonte: Elaborada pelo autor.

Tabela 3.2 - Parâmetros utilizados e resultados obtidos com algoritmos LMS-RNN.

\begin{tabular}{ccccccc}
$\tau^{*}$ & $\eta$ & $\lambda$ & iterações & tempo(s) & convergência média & MSE \\
\hline \hline $30 \mu s$ & $10^{-5}$ & $5.10^{-4}$ & 6822 & 256 & $5.10^{-4}$ & $3,98.10^{-6}$ \\
\hline $60 \mu s$ & $10^{-5}$ & $5.10^{-4}$ & 5293 & 195 & $5.10^{-4}$ & $2,87.10^{-6}$ \\
\hline $120 \mu s$ & $10^{-5}$ & $10^{-3}$ & 7031 & 287 & $5.10^{-4}$ & $3,86.10^{-6}$ \\
\hline $240 \mu s$ & $10^{-5}$ & $10^{-3}$ & 9674 & 410 & $5.10^{-4}$ & $4,54.10^{-6}$ \\
\hline $480 \mu s$ & $10^{-5}$ & $10^{-3}$ & 14772 & 561 & $5.10^{-4}$ & $4,15.10^{-6}$ \\
\hline $960 \mu s$ & $10^{-5}$ & $10^{-2}$ & 8959 & 336 & $5.10^{-4}$ & $1,20.10^{-5}$ \\
\hline $30 \mu s, 60 \mu s, 120 \mu s$, & & & & & & \\
$240 \mu s, 480 \mu s, 960 \mu s$ & $10^{-5}$ & $5.10^{-4}$ & 614 & 95 & $5.10^{-4}$ & $1,62.10^{-6}$ \\
\hline
\end{tabular}

Fonte: Elaborada pelo autor.

O problema envolvendo a característica de filtragem observado nas análises do LMS-NN deixa ser tão significativo ao incluir o termo de regularização no algoritmo, conforme observase nos resultados da tabela 3.2 e nas figuras 3.9, 3.10, 3.11, 3.12, 3.13 e 3.14. As distribuições resultantes das análises realizadas por meio do LMS-RNN com sinais individuais estão bastante próximas da distribuição simulada. A análise composta por múltiplos sinais novamente resultou 
em efeitos notavelmente positivos considerando tempo de convergência do algoritmo, numero de iterações e erro médio quadrático em relação a distribuição simulada.

\subsubsection{Hopfield}

Abaixo seguem os gráficos das distribuições alcançadas por meio do algoritmo da rede recorrente de Hopfield para cada um dos seis sinais de decaimento simulados conforme descrito no inicio da sessão. Os parâmetros utilizados na análise com cada um dos sinais, além do erro médio quadrático da resposta em relação a distribuição simulada estão relacionados na tabela 3.3.

Figura 3.16 - Distribuição obtida através do algoritmo da rede recorrente de Hopfield para o sinal simulado com $\tau^{*}=30 \mu \mathrm{s}$ e comparado à distribuição simulada do teste um.

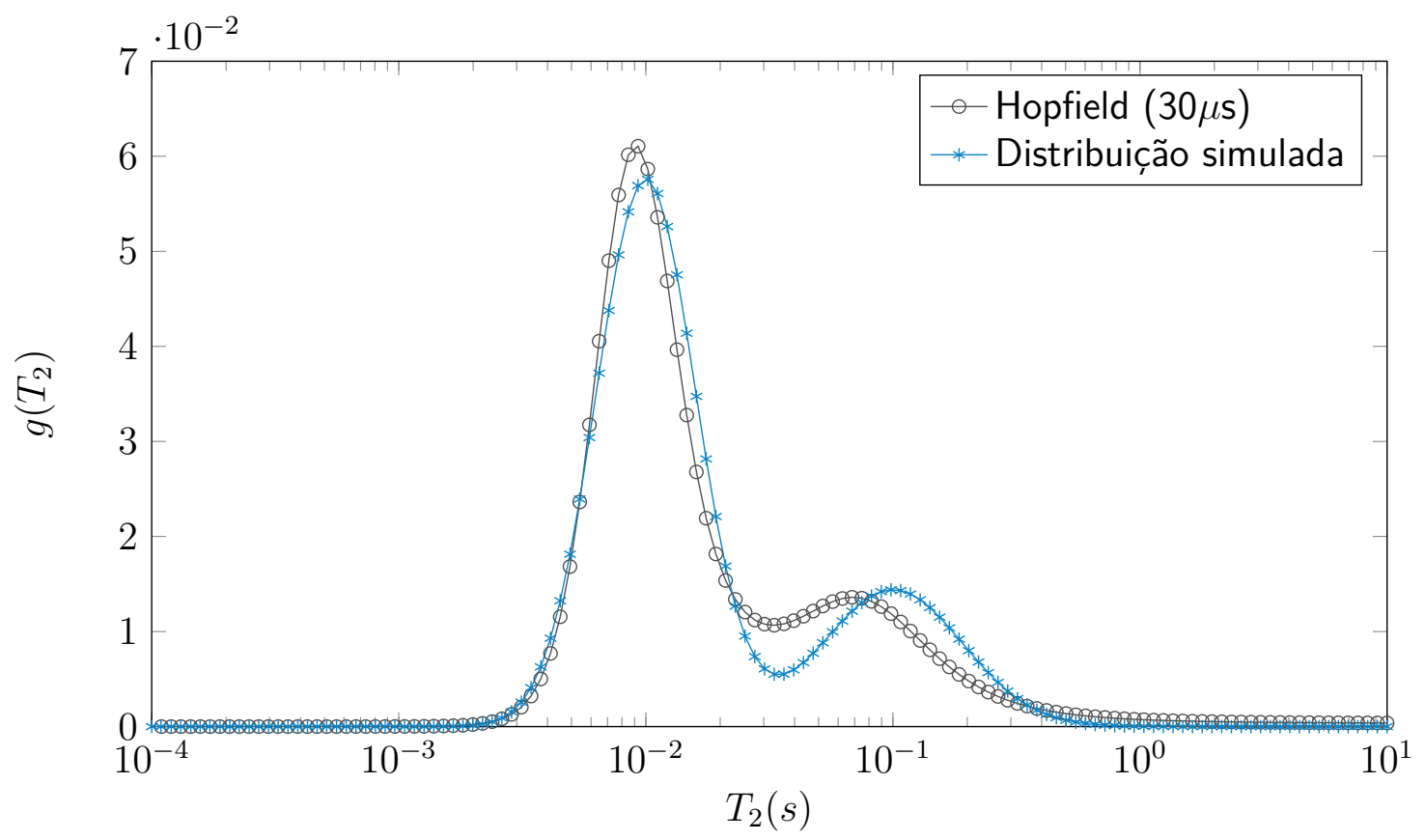

Fonte: Elaborada pelo autor. 
Figura 3.17 - Distribuição obtida através do algoritmo da rede recorrente de Hopfield para o sinal simulado com $\tau^{*}=60 \mu \mathrm{s}$ e comparado à distribuição simulada do teste um.

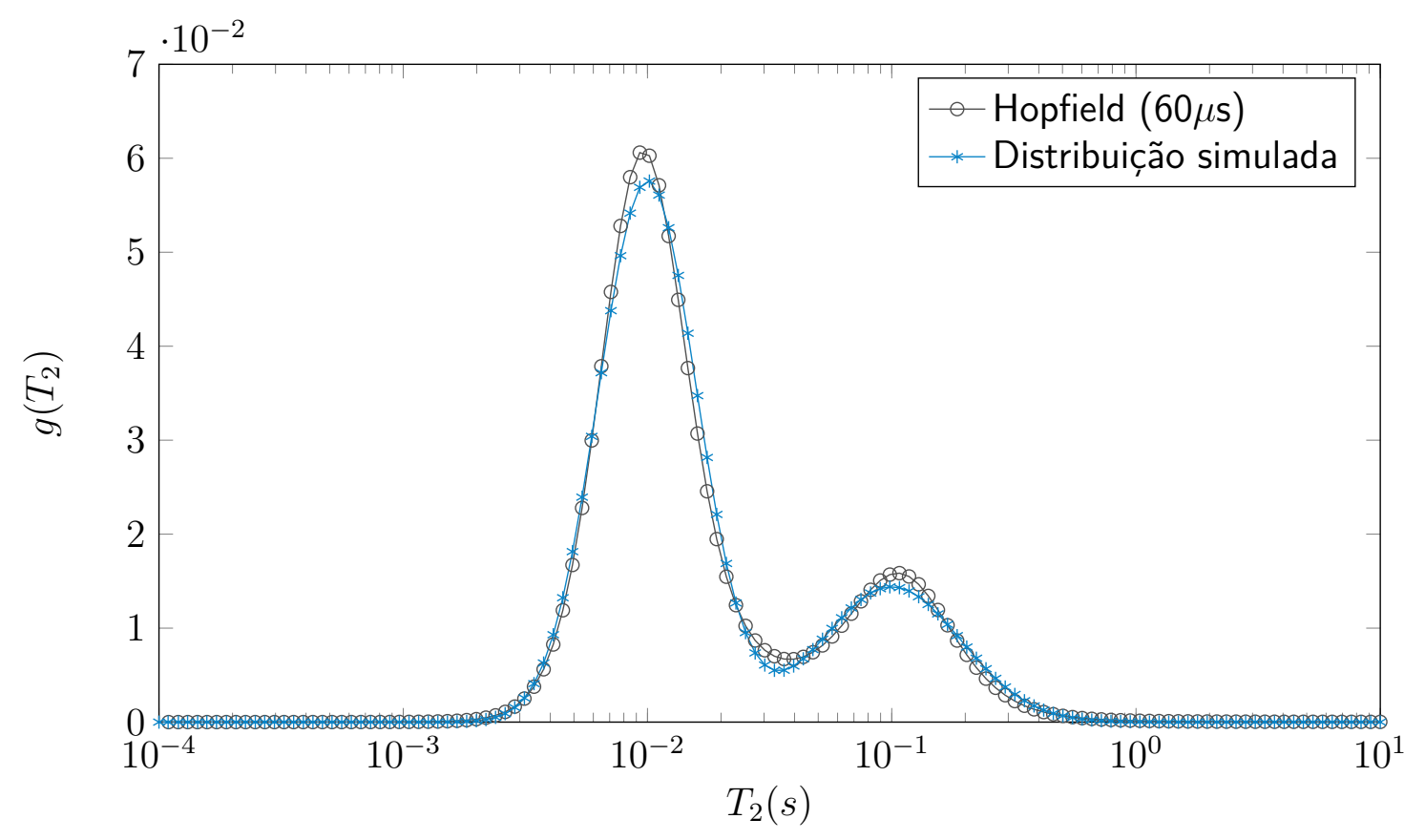

Fonte: Elaborada pelo autor.

Figura 3.18 - Distribuição obtida através do algoritmo da rede recorrente de Hopfield para o sinal simulado com $\tau^{*}=120 \mu \mathrm{s}$ e comparado à distribuição simulada do teste um.

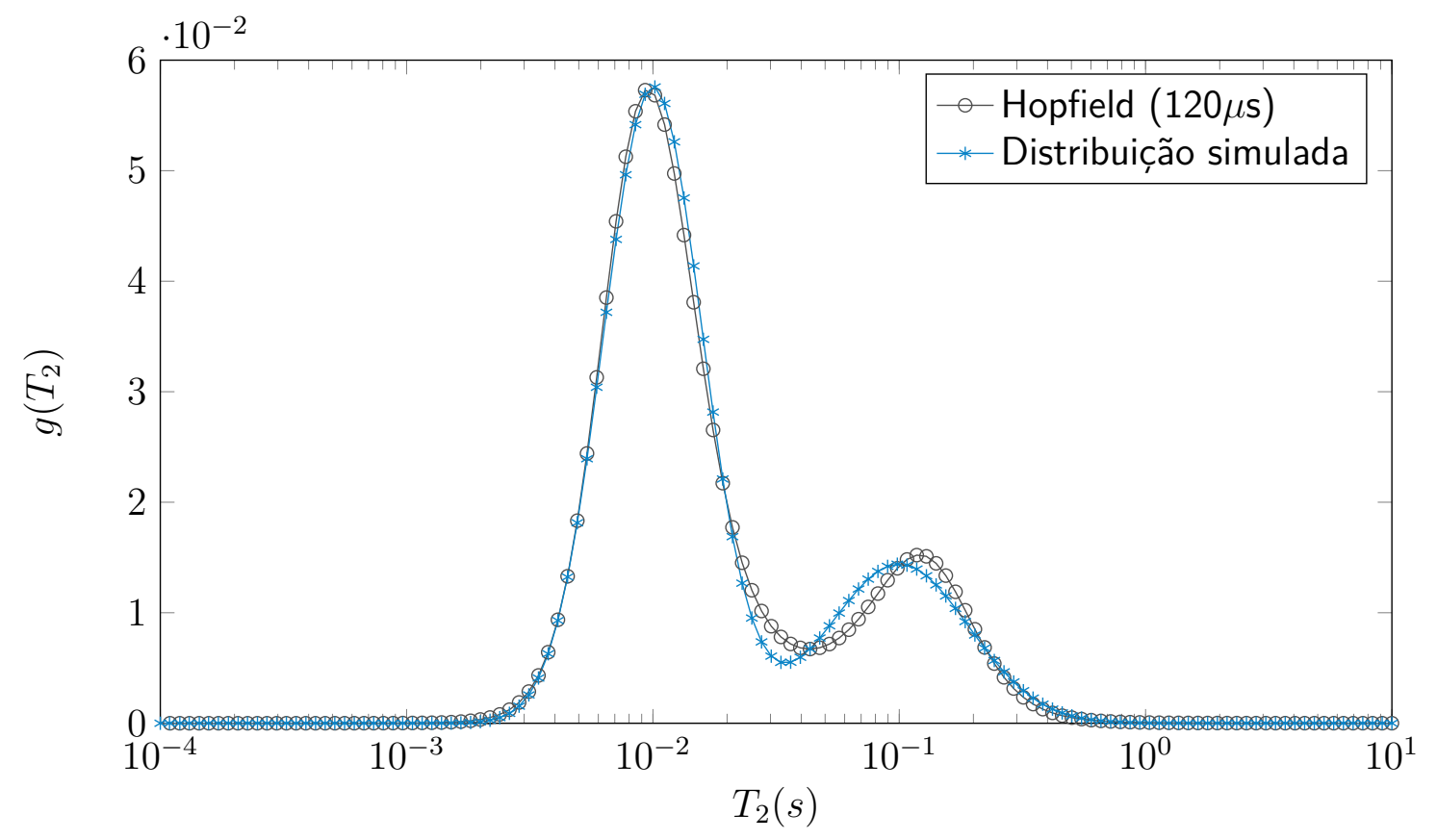

Fonte: Elaborada pelo autor. 
Figura 3.19 - Distribuição obtida através do algoritmo da rede recorrente de Hopfield para o sinal simulado com $\tau^{*}=240 \mu \mathrm{s}$ e comparado à distribuição simulada do teste um.

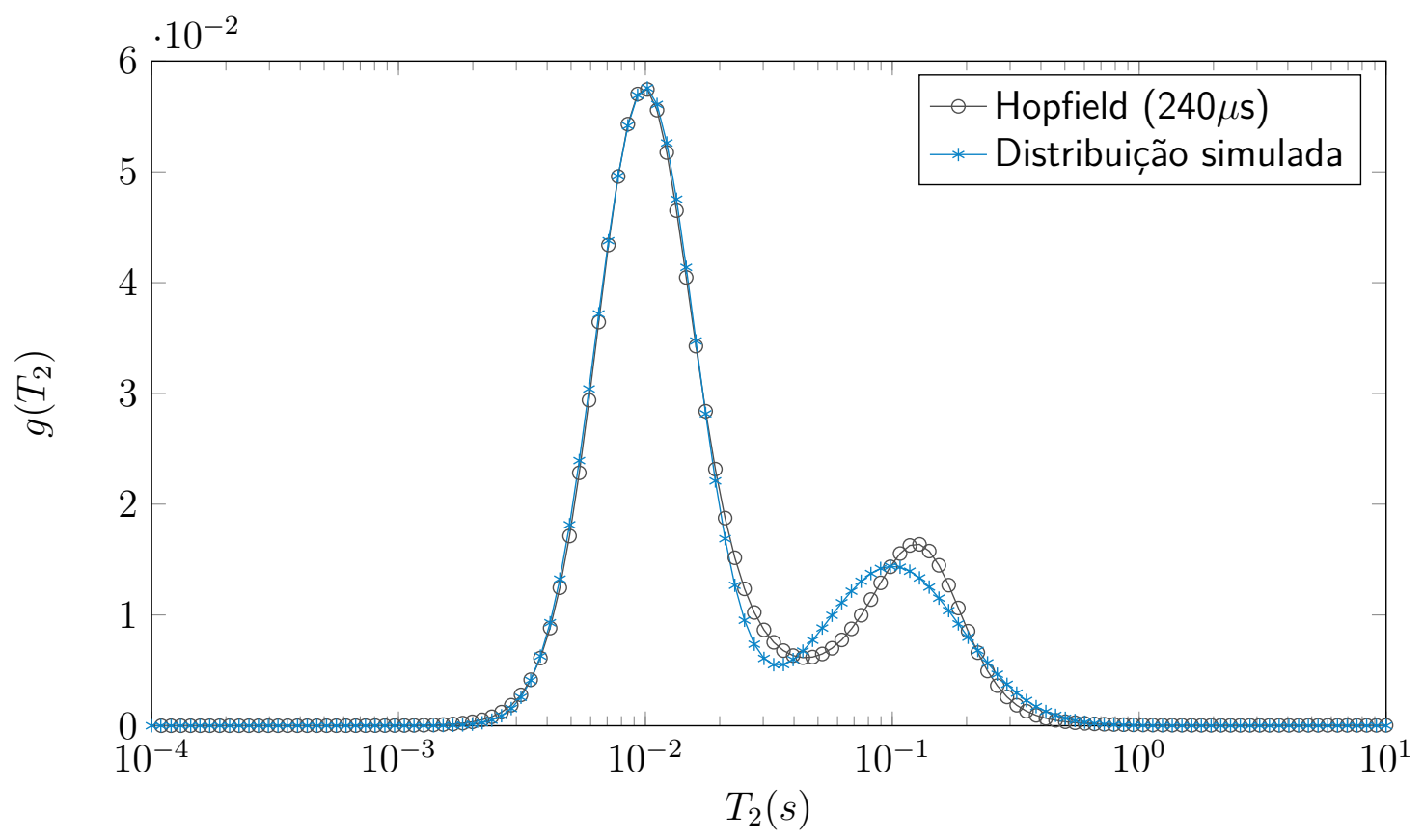

Fonte: Elaborada pelo autor.

Figura 3.20 - Distribuição obtida através do algoritmo da rede recorrente de Hopfield para o sinal simulado com $\tau^{*}=480 \mu \mathrm{s}$ e comparado à distribuição simulada do teste um.

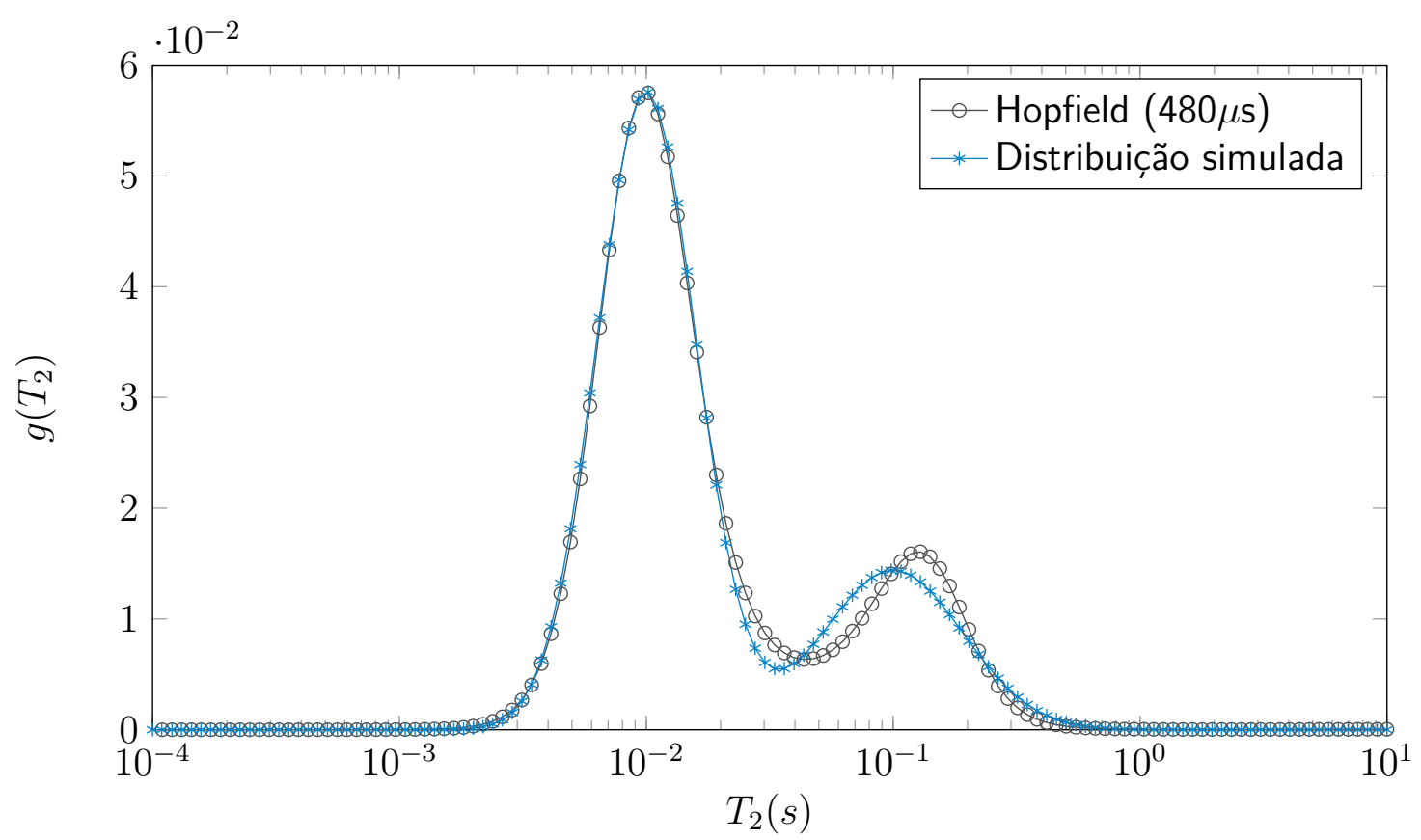

Fonte: Elaborada pelo autor. 
Figura 3.21 - Distribuição obtida através do algoritmo da rede recorrente de Hopfield para o sinal simulado com $\tau^{*}=960 \mu \mathrm{s}$ e comparado à distribuição simulada do teste um.

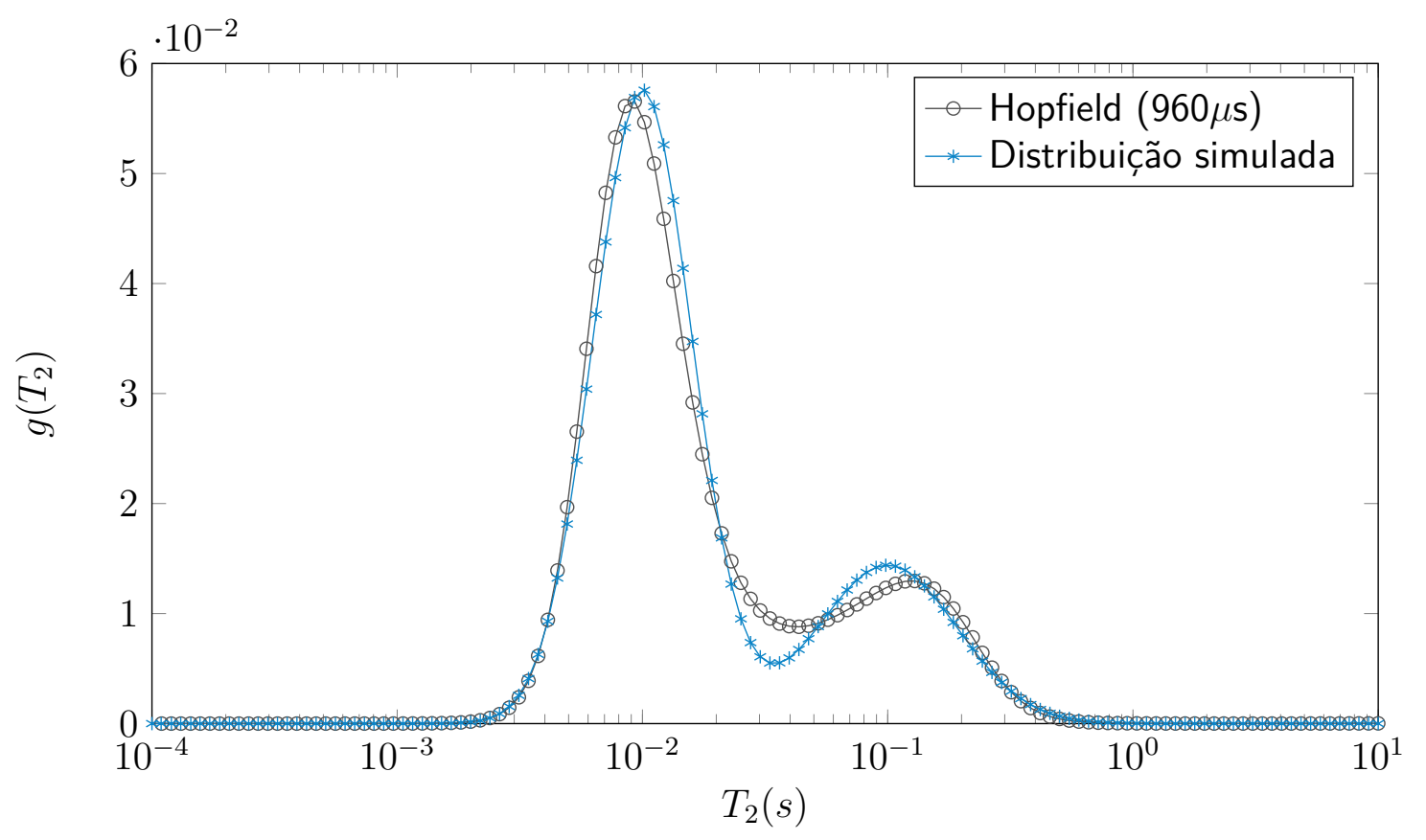

Fonte: Elaborada pelo autor.

Tabela 3.3 - Parâmetros utilizados e resultados obtidos com algoritmo de Hopfield.

\begin{tabular}{cccccc}
$\tau^{*}$ & $h$ & iterações & tempo(s) & convergência média & MSE \\
\hline \hline $30 \mu s$ & $10^{-3}$ & 33954 & 410 & $10^{-4}$ & $6,25.10^{-6}$ \\
\hline $60 \mu s$ & $10^{-3}$ & 15897 & 195 & $10^{-4}$ & $1,10.10^{-6}$ \\
\hline $120 \mu s$ & $10^{-3}$ & 22949 & 286 & $10^{-4}$ & $1,11.10^{-6}$ \\
\hline $240 \mu s$ & $10^{-3}$ & 28699 & 392 & $10^{-4}$ & $1,20.10^{-6}$ \\
\hline $480 \mu s$ & $10^{-3}$ & 48656 & 772 & $10^{-4}$ & $1,16.10^{-6}$ \\
\hline $960 \mu s$ & $10^{-3}$ & 99335 & 1486 & $10^{-4}$ & $3,40.10^{-6}$ \\
\hline
\end{tabular}

Fonte: Elaborada pelo autor.

O algoritmo da rede recorrente de Hopfield praticamente não foi afetado pela variação do $\tau^{*}$ considerando os sinais formados pela distribuição com picos próximos. As repostas se mantiveram regulares com todos os seis sinais de decaimento com resultado final bastante próximo da distribuição simulada. Nota-se, entretanto, que a quantidade de iterações necessárias para convergência das redes recorrentes de Hopfield é maior em relação ao LMS-NN e LMS-RNN. 


\subsubsection{LS-R (SVD)}

A tabela 3.4 contém os parâmetros utilizados na análise com cada um dos sinais de decaimento simulados, além do erro médio quadrático da resposta em relação a distribuição simulada. As figuras 3.22, 3.23, 3.24, 3.25, 3.26 e 3.27 comparam os resultados obtidos com algoritmo em relação a distribuição simulada para o teste um.

Figura 3.22 - Distribuição obtida através do algoritmo LS-R para o sinal simulado com $\tau^{*}=30 \mu \mathrm{s}$ e comparado à distribuição simulada do teste um.

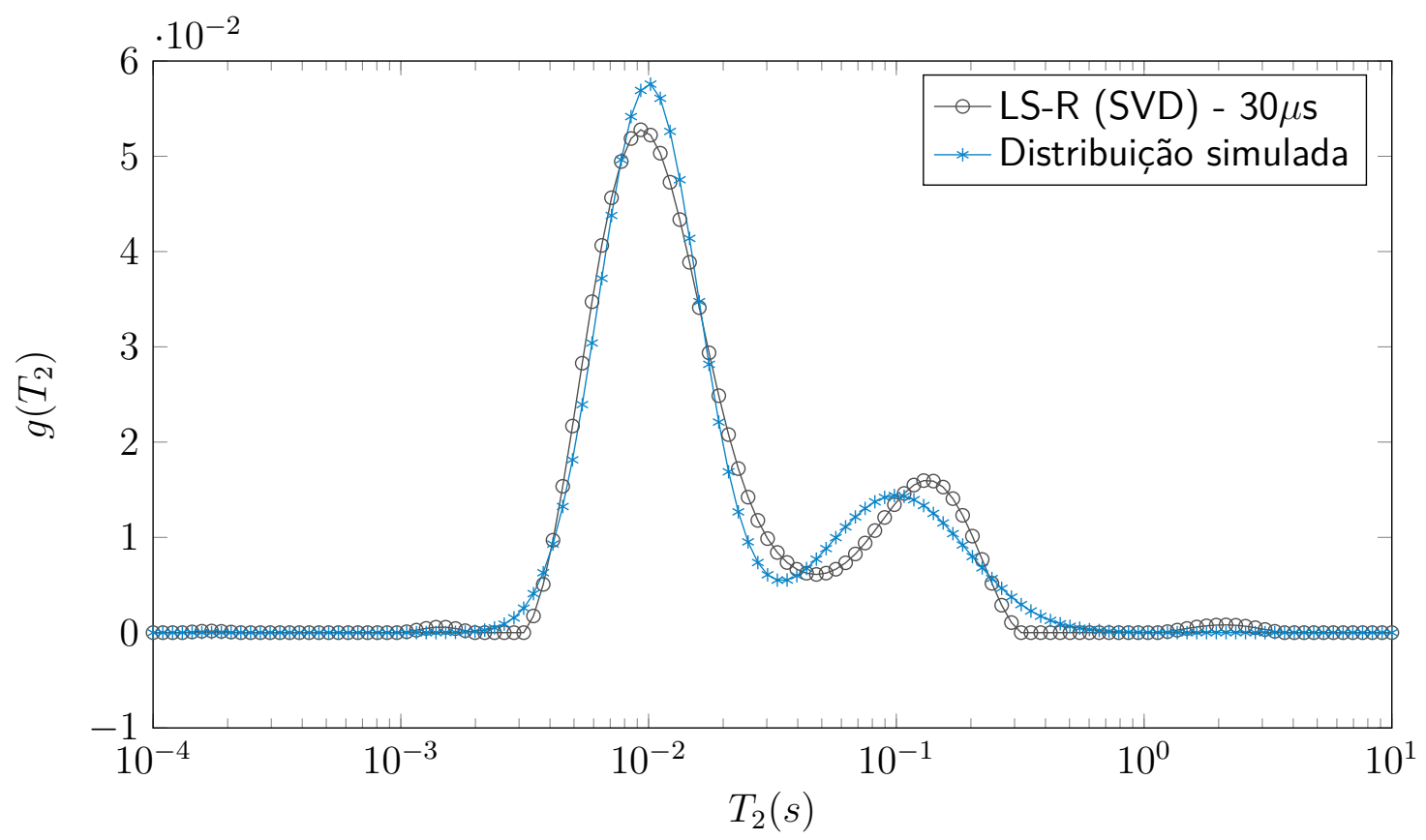

Fonte: Elaborada pelo autor. 
Figura 3.23 - Distribuição obtida através do algoritmo LS-R para o sinal simulado com $\tau^{*}=60 \mu \mathrm{s}$ e comparado à distribuição simulada do teste um.

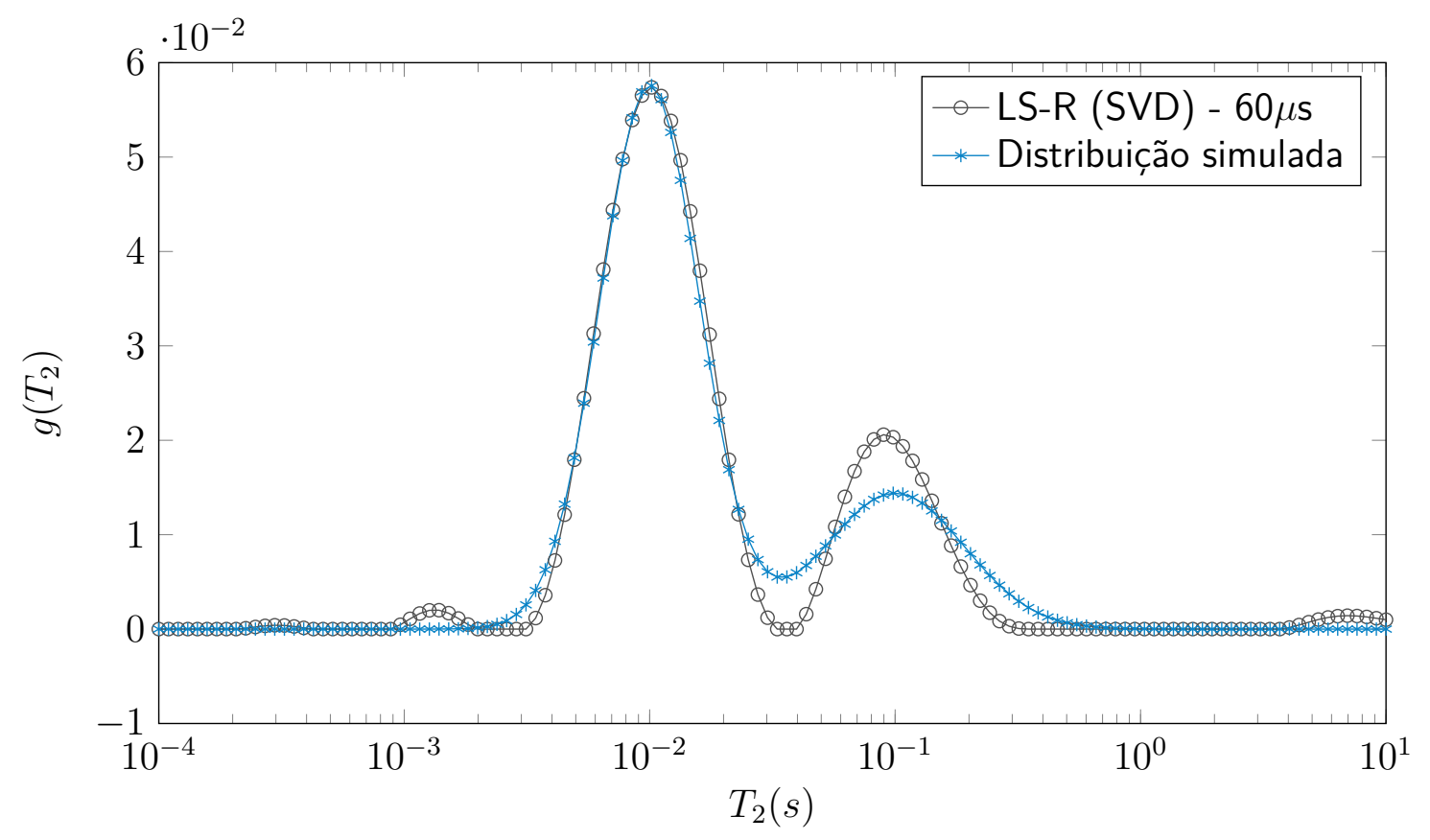

Fonte: Elaborada pelo autor.

Figura 3.24 - Distribuição obtida através do algoritmo LS-R para o sinal simulado com $\tau^{*}=120 \mu \mathrm{s}$ e comparado à distribuição simulada do teste um.

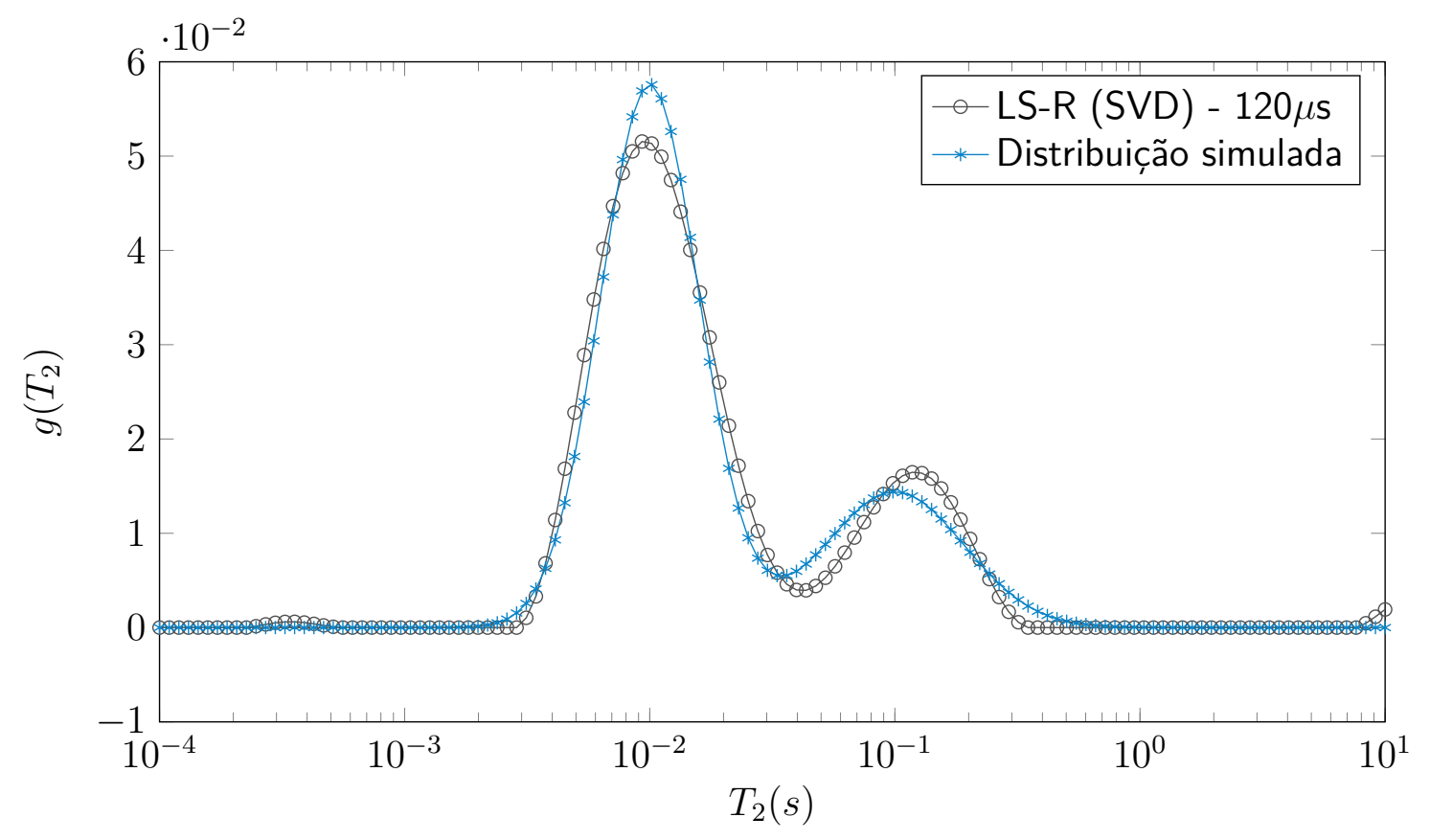

Fonte: Elaborada pelo autor. 
Figura 3.25 - Distribuição obtida através do algoritmo LS-R para o sinal simulado com $\tau^{*}=240 \mu \mathrm{s}$ e comparado à distribuição simulada do teste um.

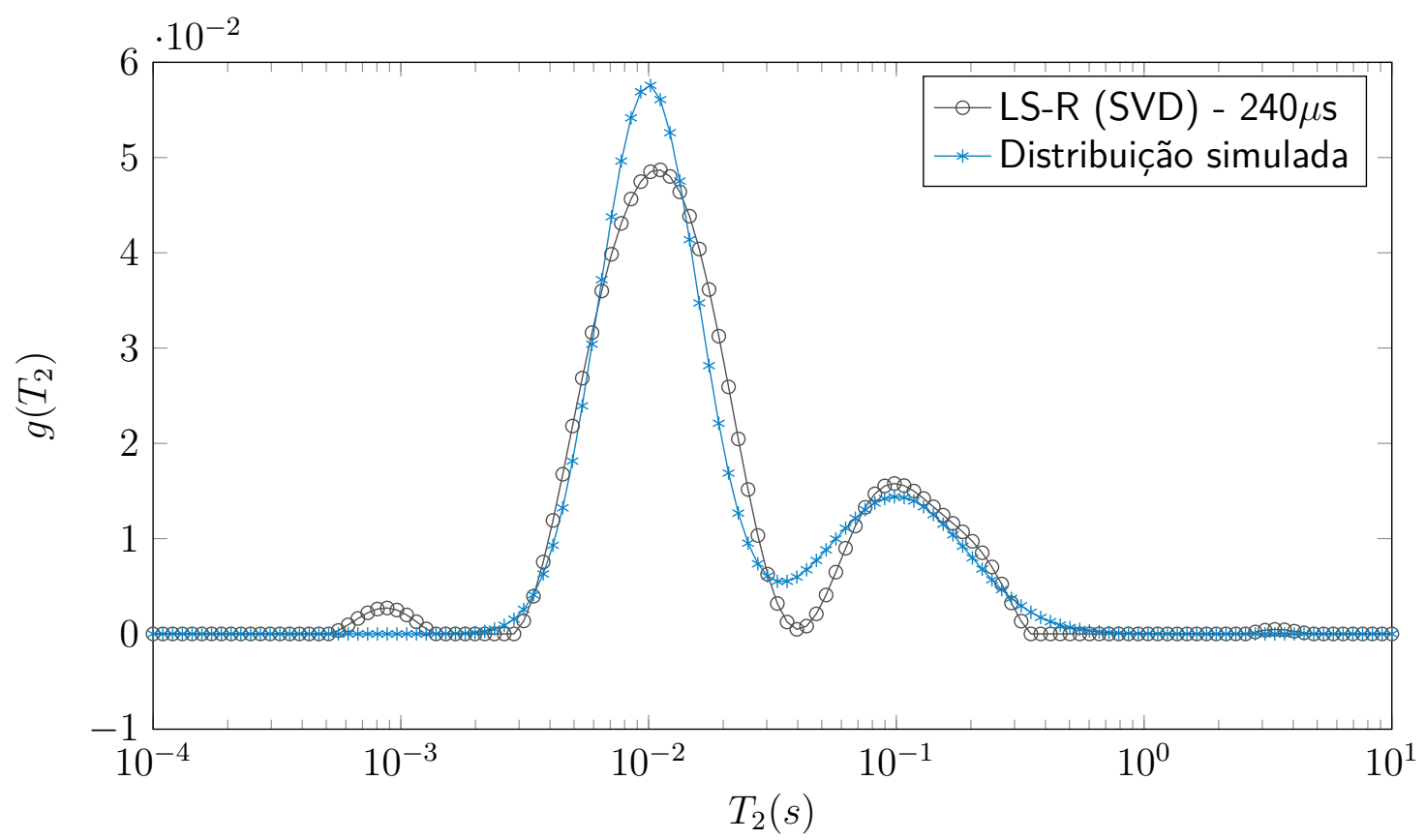

Fonte: Elaborada pelo autor.

Figura 3.26 - Distribuição obtida através do algoritmo LS-R para o sinal simulado com $\tau^{*}=480 \mu \mathrm{s}$ e comparado à distribuição simulada do teste um.

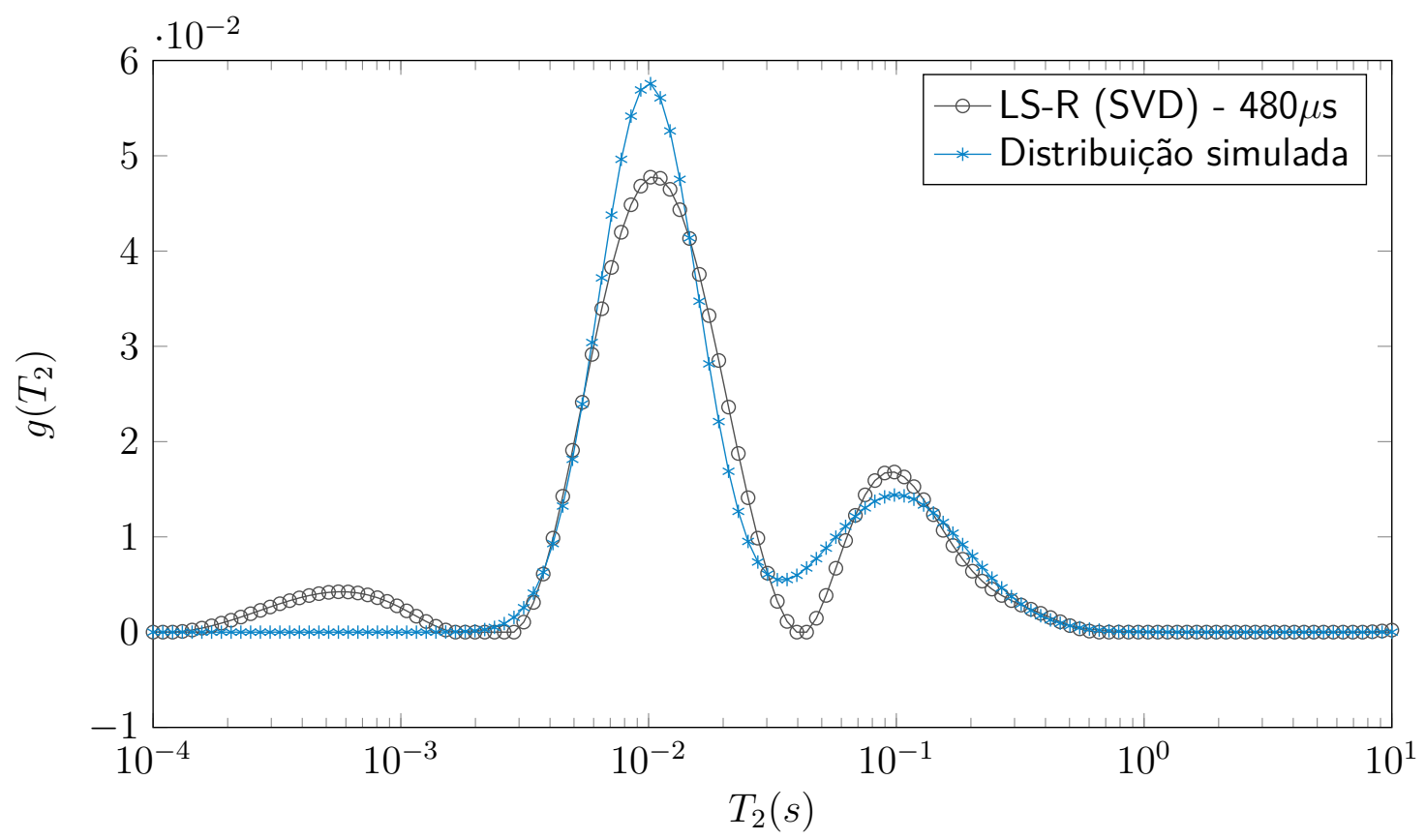

Fonte: Elaborada pelo autor. 
Figura 3.27 - Distribuição obtida através do algoritmo LS-R para o sinal simulado com $\tau^{*}=960 \mu \mathrm{s}$ e comparado à distribuição simulada do teste um.

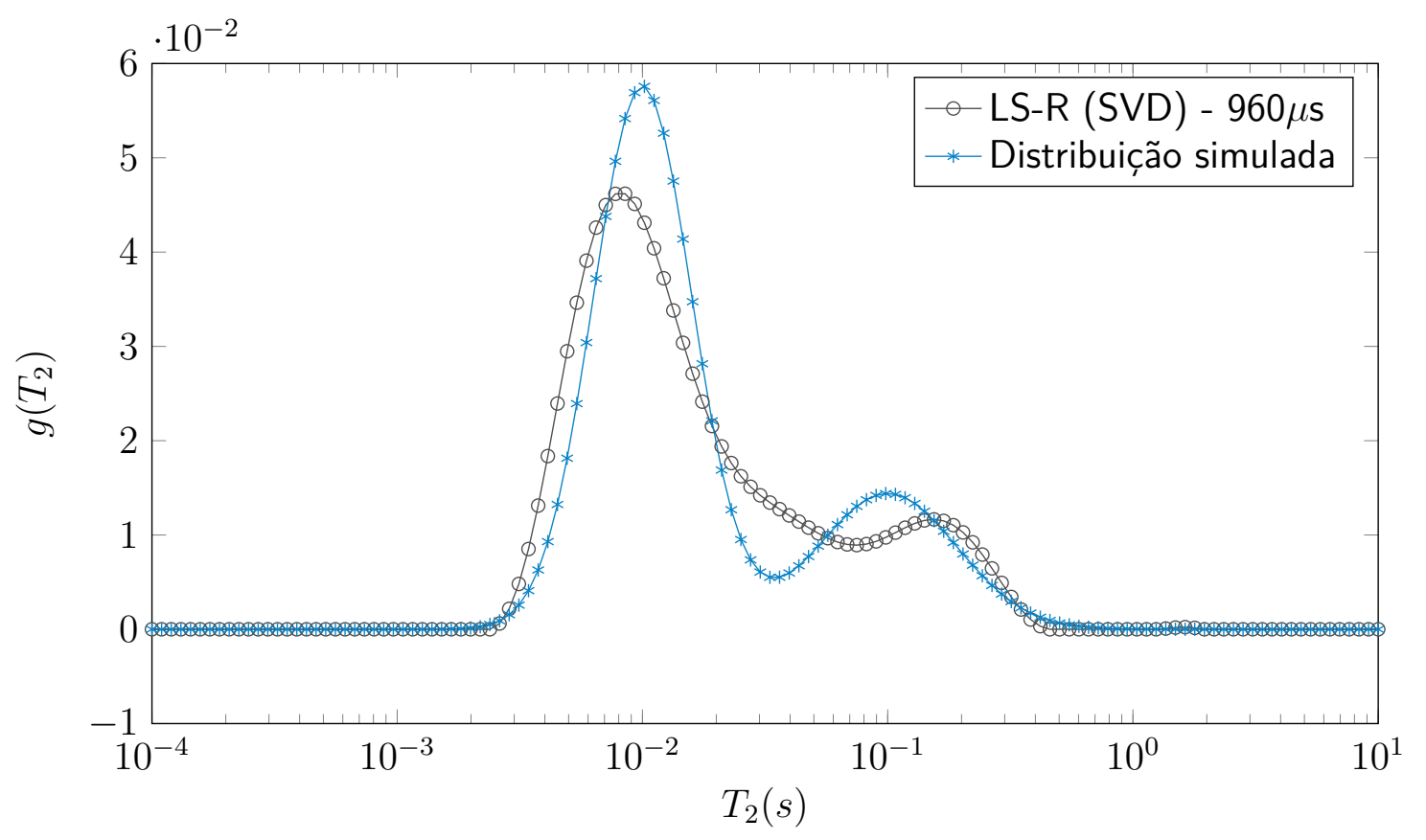

Fonte: Elaborada pelo autor.

Tabela 3.4 - Parâmetros utilizados e resultados obtidos com algoritmos LS-R.

\begin{tabular}{cccccc}
$\tau^{*}$ & $\lambda$ & iterações & tempo(s) & MSE & $\sum\left(g\left(T_{2}\right)<0\right)^{2}$ \\
\hline \hline $30 \mu s$ & 10 & 673 & 65 & $4,02.10^{-6}$ & $10^{-8}$ \\
\hline $60 \mu s$ & 10 & 807 & 80 & $4,74.10^{-6}$ & $10^{-8}$ \\
\hline $120 \mu s$ & 10 & 786 & 78 & $3,95.10^{-6}$ & $10^{-8}$ \\
\hline $240 \mu s$ & 10 & 730 & 74 & $8,04.10^{-6}$ & $10^{-8}$ \\
\hline $480 \mu s$ & 10 & 789 & 82 & $8,82.10^{-6}$ & $10^{-8}$ \\
\hline $960 \mu s$ & 10 & 795 & 90 & $1,92.10^{-5}$ & $10^{-8}$ \\
\hline
\end{tabular}

Fonte: Elaborada pelo autor.

No geral o algoritmo dos mínimos quadrados regularizado se comporta bem, os picos da distribuição estão bem definidos para todos os seis sinais analisados, contudo em alguns casos, como no gráfico 3.23, nota-se ainda o aparecimento de oscilações indesejadas. Infelizmente a escolha de um parâmetro de regularização ideal é uma tarefa bastante complicada e um valor maior para $\lambda$ poderia causar uma suavização excessiva na resposta. 
Abaixo segue quadro comparativo entre os melhores resultados (em relação ao erro médio quadrático) considerando os quatro algoritmos no primeiro teste:

Tabela 3.5 - Comparativo entre os melhores resultados obtidos com cada algoritmo no primeiro teste.

\begin{tabular}{ccccc} 
algoritmo & MSE & iterações & tempo(s) & $\sum\left(g\left(T_{2}\right)<0\right)^{2}$ \\
\hline \hline LMS-NN (múltiplos sinais) & $3,47.10^{-6}$ & 510 & 80 & 0 \\
\hline LMS-RNN (múltiplos sinais) & $1,62.10^{-6}$ & 614 & 95 & 0 \\
\hline Hopfield $(60 \mu s)$ & $1,10.10^{-6}$ & 15897 & 195 & 0 \\
\hline LS-R $(60 \mu s)$ & $3,95.10^{-6}$ & 786 & 78 & $10^{-8}$ \\
\hline
\end{tabular}

Fonte: Elaborada pelo autor.

\subsection{Teste 2 - distribuições simuladas espaçadas}

O segundo teste foi também realizado utilizando distribuição de valores $T_{2}$ simulada através da distribuição multi-log normal formada por $2^{16}$ pontos espaçados logaritmicamente no intervalo de $10^{-4} \mathrm{~s}$ à $10^{1} \mathrm{~s}$, entretanto formada agora por dois picos espaçados e centrados em $10^{-3}$ segundos e 1 segundo. A partir da distribuição, procedendo como no experimento anterior, foram obtidas 6 diferentes simulações de sinais de magnetização com diferentes tempos de eco $\tau$ contendo $2^{12}$ pontos cada e contaminados com ruído branco.

O objetivo deste deste teste é analisar o comportamento do algoritmo frente a situações onde a característica de filtragem do núcleo da equação de Fredholm é acentuada tendo em vista o espaçamento acentuado entre os picos.

\subsubsection{LMS-NN}

Abaixo estão os gráficos das respostas do algoritmo LMS-NN para os seis sinais simulados, além da resposta considerando o conjunto de sinais. A tabela 3.6 contém os parâmetros utilizados nos testes e os resultados alcançados. 
Figura 3.28 - Distribuição obtida através do algoritmo LMS-NN para o sinal simulado com $\tau^{*}=$ $30 \mu s$ e comparado à distribuição simulada do teste dois.

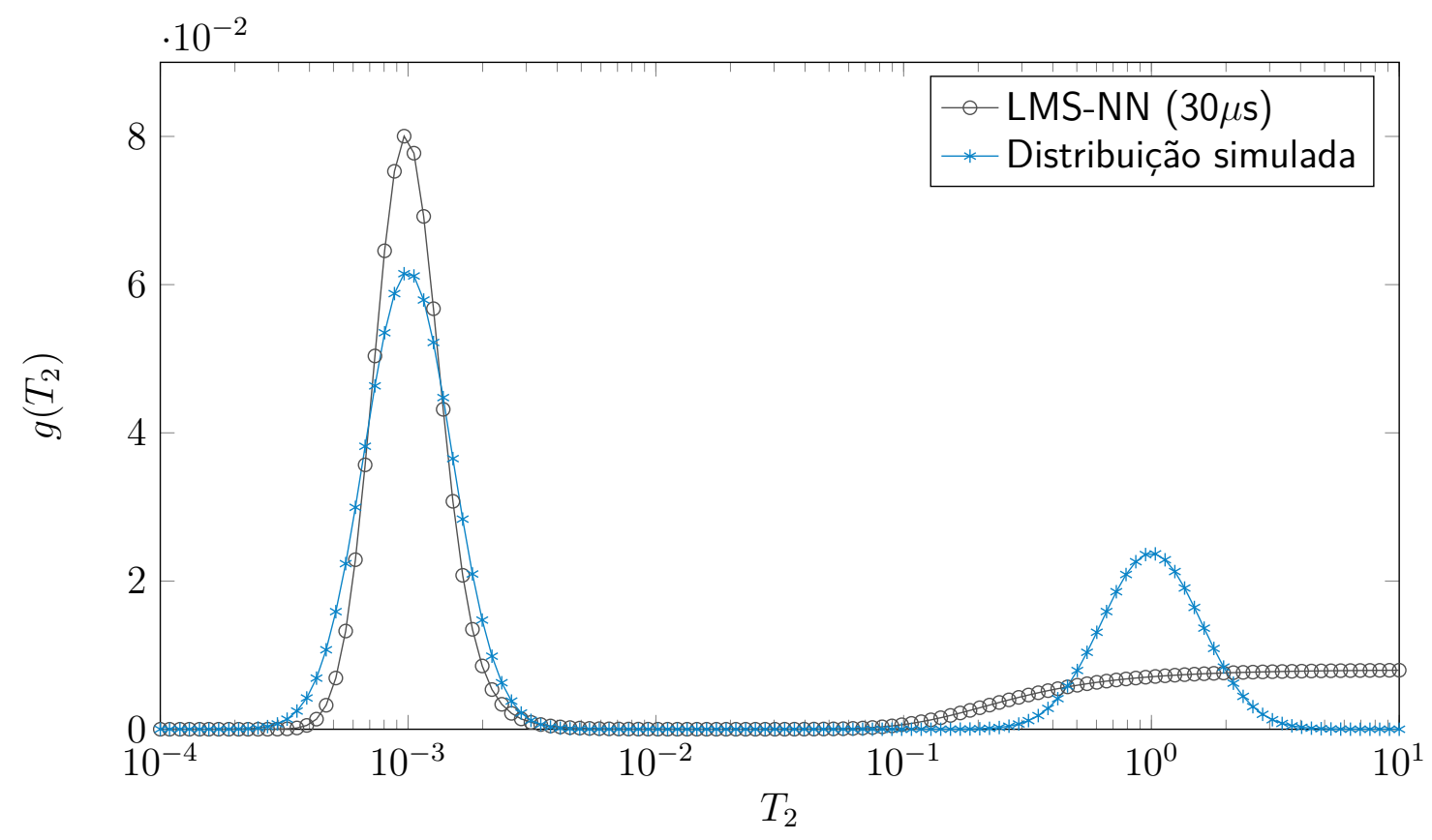

Fonte: Elaborada pelo autor.

Figura 3.29 - Distribuição obtida através do algoritmo LMS-NN para o sinal simulado $\operatorname{com} \tau^{*}=$ $60 \mu s$ e comparado à distribuição simulada do teste dois.

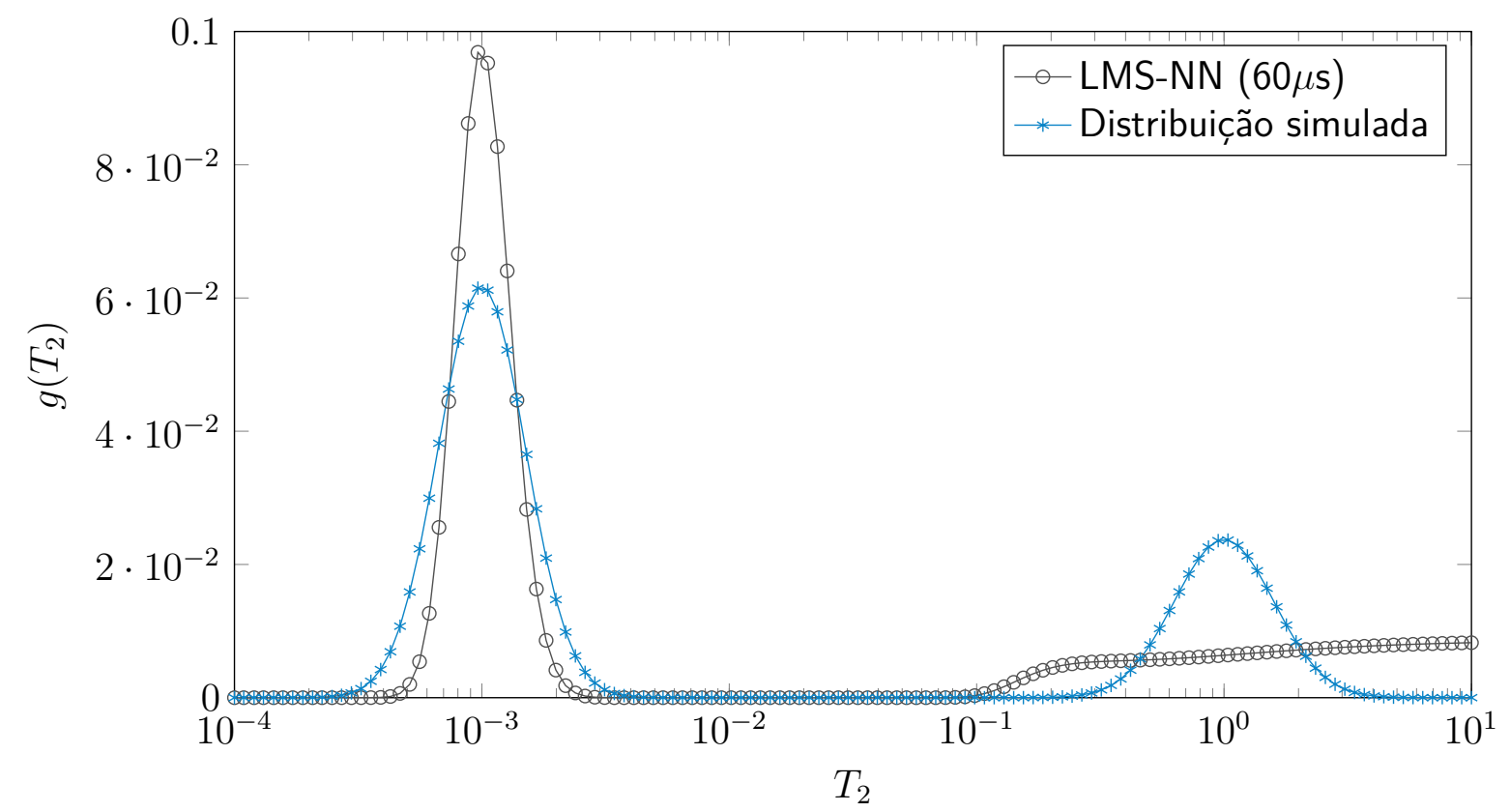

Fonte: Elaborada pelo autor. 
Figura 3.30 - Distribuição obtida através do algoritmo LMS-NN para o sinal simulado com $\tau^{*}=$ $120 \mu \mathrm{s}$ e comparado à distribuição simulada do teste dois.

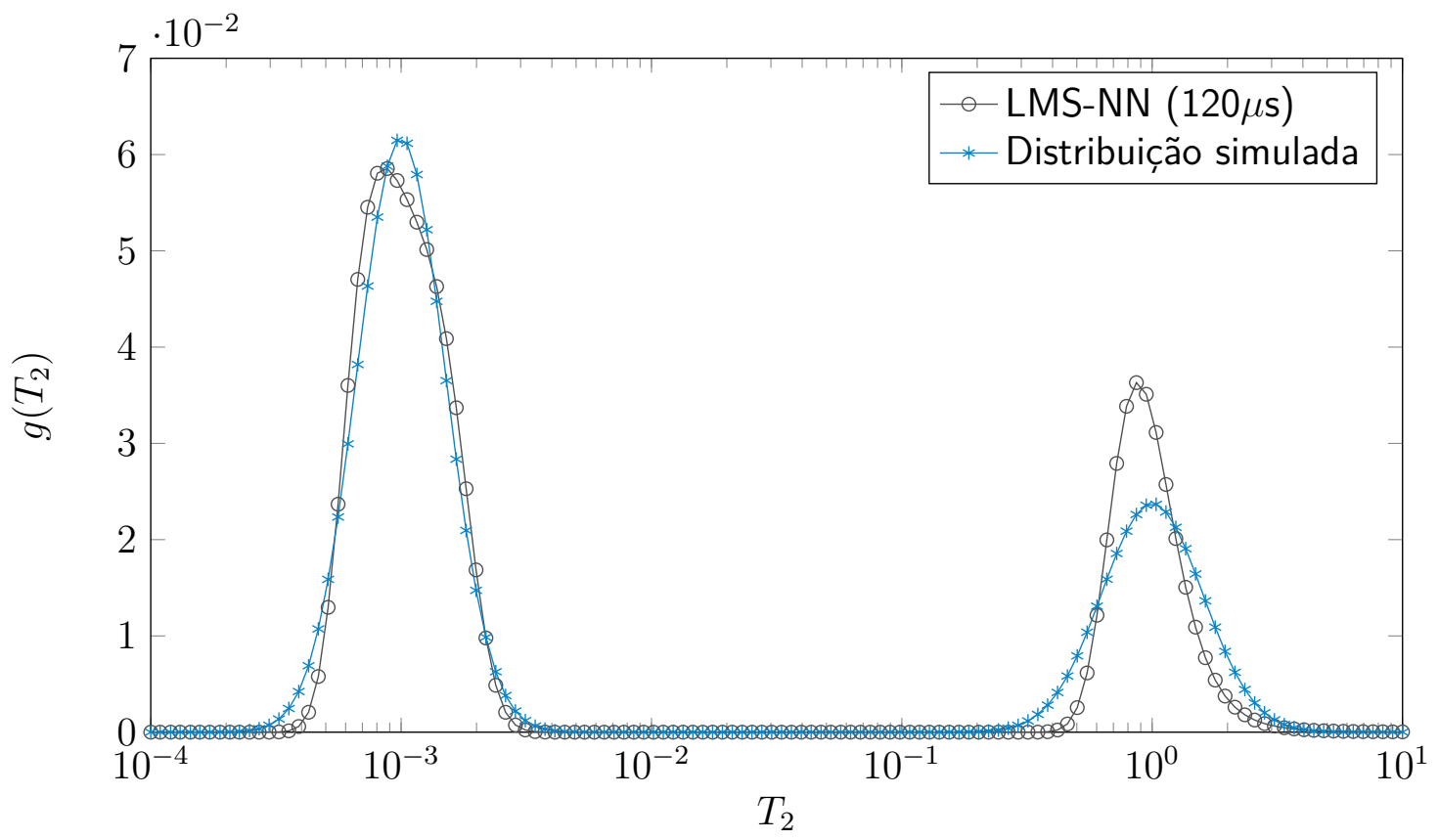

Fonte: Elaborada pelo autor.

Figura 3.31 - Distribuição obtida através do algoritmo LMS-NN para o sinal simulado com $\tau^{*}=$ $240 \mu \mathrm{s}$ e comparado à distribuição simulada do teste dois.

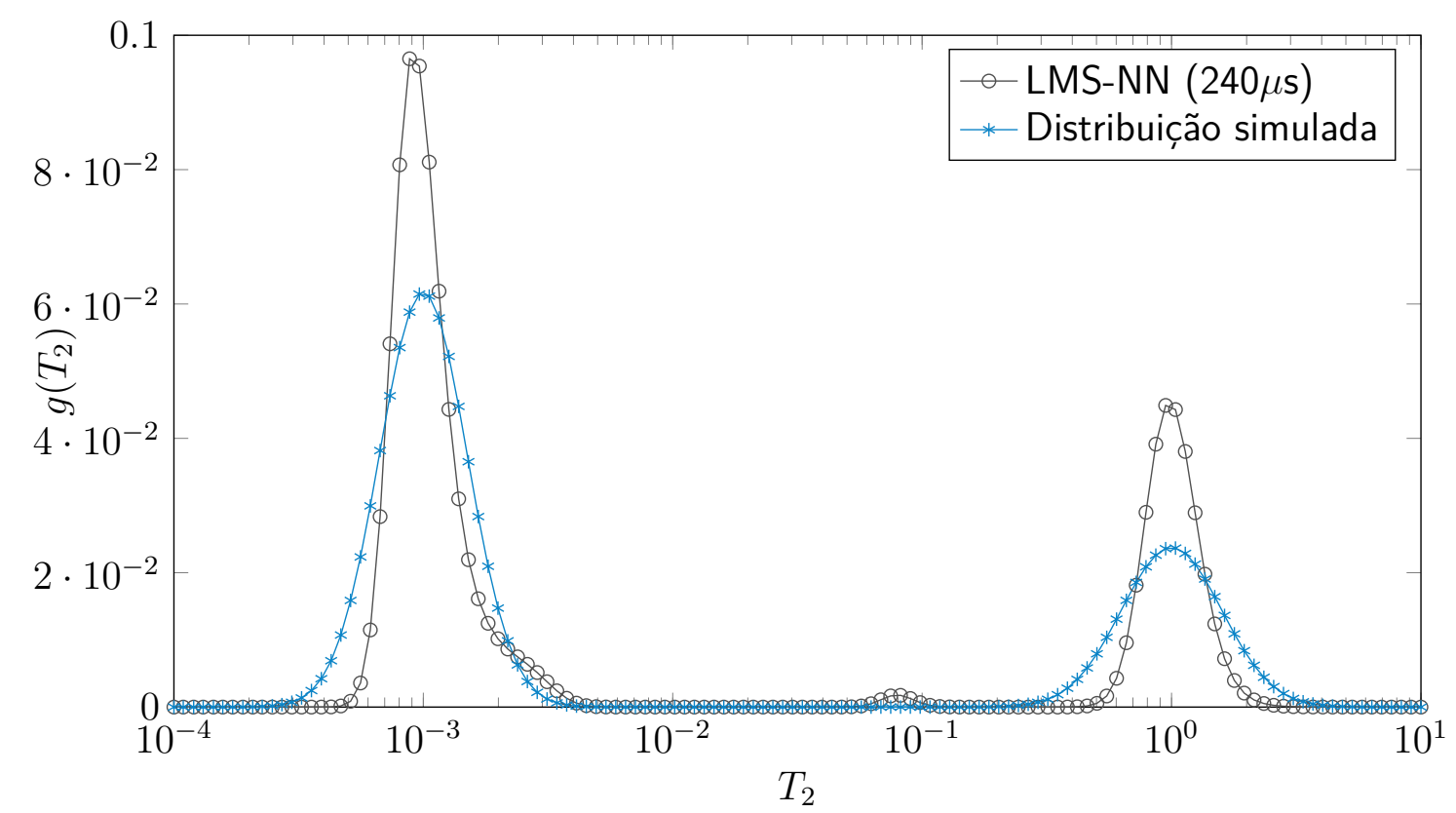

Fonte: Elaborada pelo autor. 
Figura 3.32 - Distribuição obtida através do algoritmo LMS-NN para o sinal simulado com $\tau^{*}=$ $480 \mu s$ e comparado à distribuição simulada do teste dois.

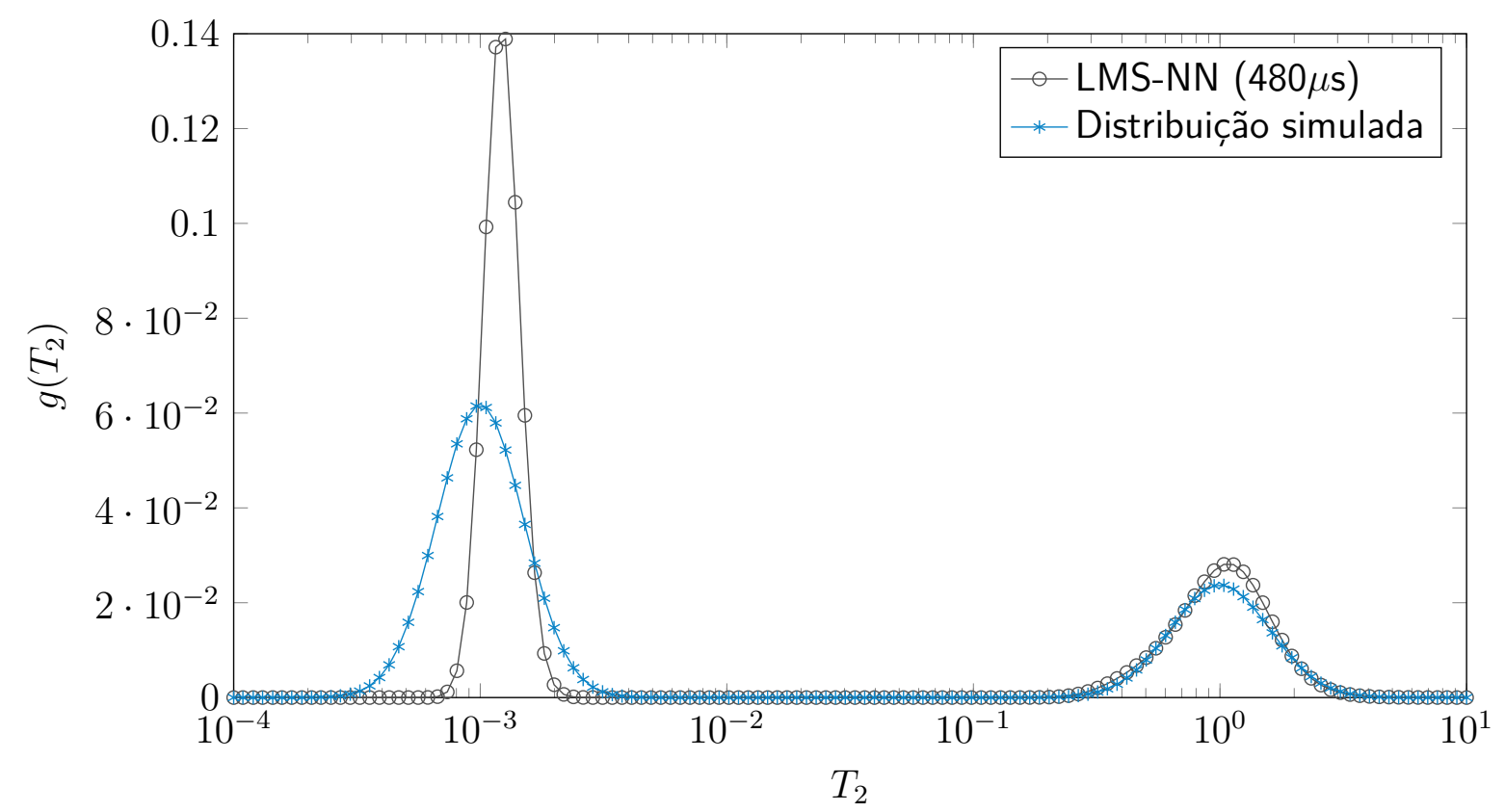

Fonte: Elaborada pelo autor.

Figura 3.33 - Distribuição obtida através do algoritmo LMS-NN para o sinal simulado com $\tau^{*}=$ $960 \mu s$ e comparado à distribuição simulada do teste dois.

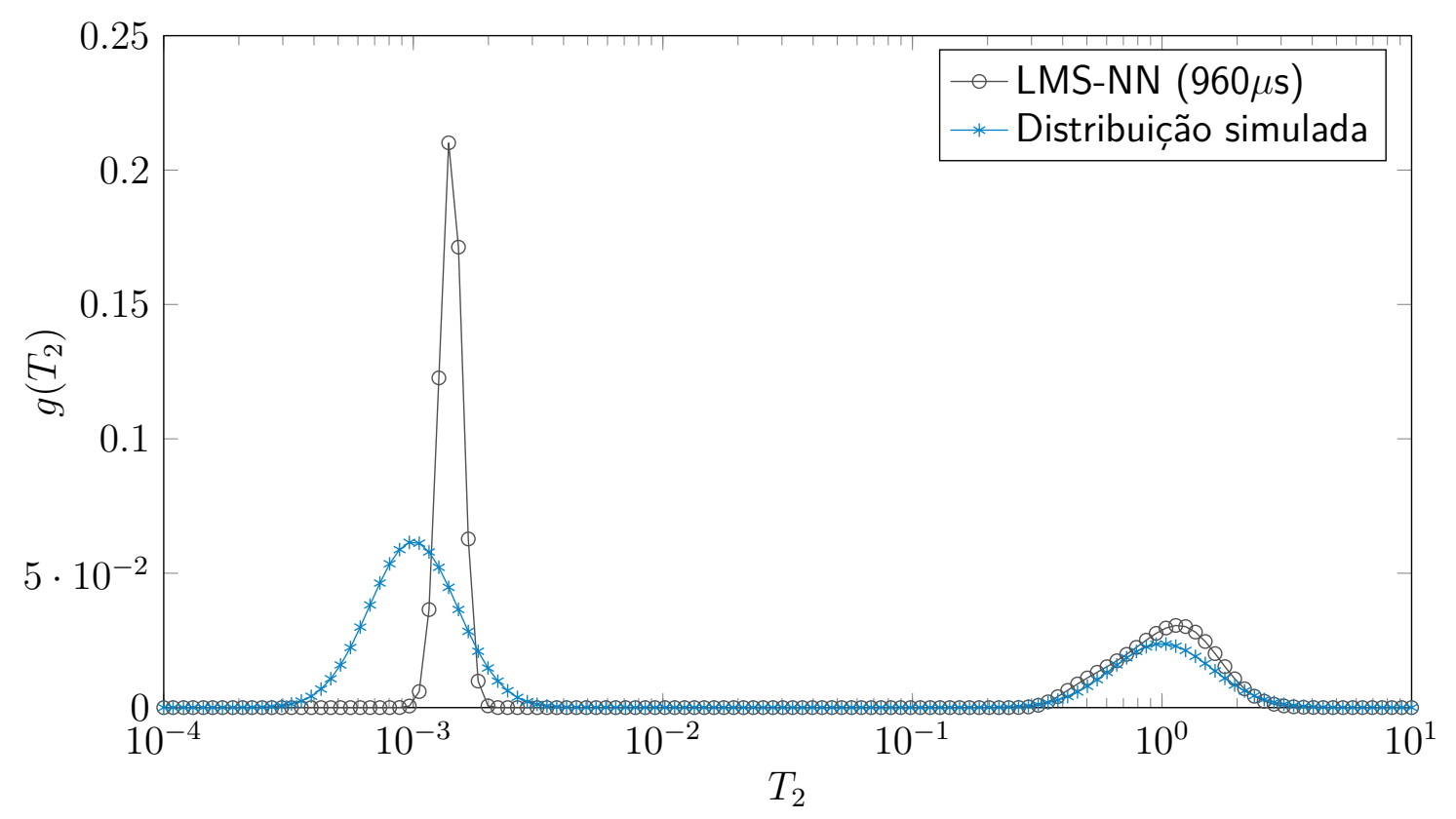

Fonte: Elaborada pelo autor. 
Figura 3.34 - Distribuição obtida através do algoritmo LMS-NN combinando os sinais simulados com $\tau^{*}=30 \mu s, 60 \mu s, 120 \mu s, 240 \mu s, 480 \mu s$ e $960 \mu s$ e comparado à distribuição simulada.

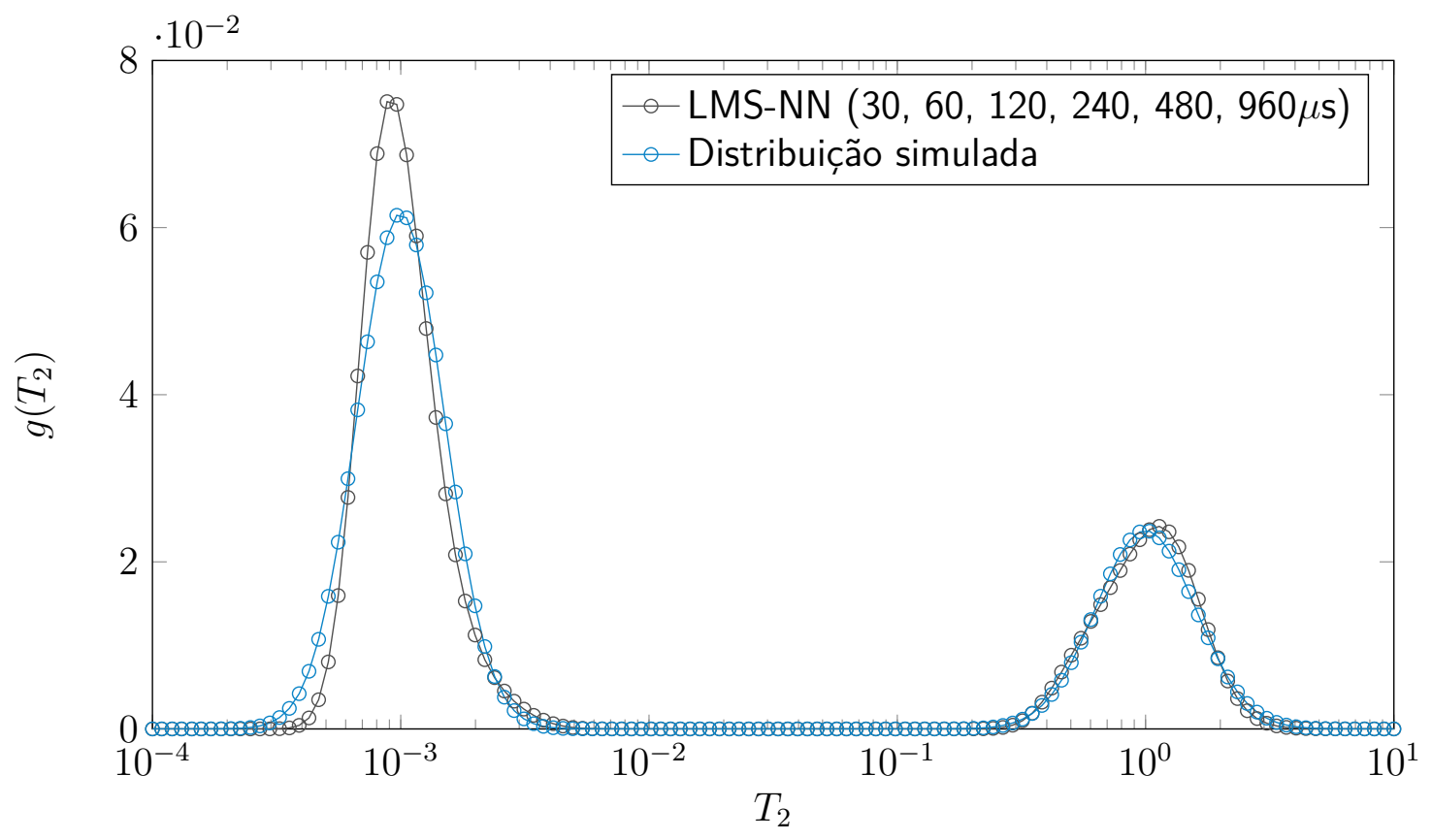

Fonte: Elaborada pelo autor.

Tabela 3.6 - Parâmetros utilizados e resultados obtidos com algoritmos LMS-NN para o teste dois.

\begin{tabular}{cccccc}
$\tau^{*}$ & $\eta$ & iterações & tempo(s) & convergência média & MSE \\
\hline \hline $30 \mu s$ & $10^{-5}$ & 7135 & 201 & $5.10^{-4}$ & $3,69.10^{-5}$ \\
\hline $60 \mu s$ & $10^{-5}$ & 12347 & 325 & $5.10^{-4}$ & $7,12.10^{-5}$ \\
\hline $120 \mu s$ & $10^{-5}$ & 31829 & 864 & $5.10^{-4}$ & $1,06.10^{-5}$ \\
\hline $240 \mu s$ & $10^{-5}$ & 40479 & 1101 & $5.10^{-4}$ & $6,06.10^{-5}$ \\
\hline $480 \mu s$ & $10^{-5}$ & 26242 & 699 & $5.10^{-4}$ & $2,72.10^{-5}$ \\
\hline $960 \mu s$ & $10^{-5}$ & 61445 & 2146 & $5.10^{-4}$ & $5,60.10^{-5}$ \\
\hline $30 \mu s, 60 \mu s, 120 \mu s$, & & & & & \\
$240 \mu s, 480 \mu s, 960 \mu s$ & $10^{-5}$ & 5425 & 830 & $5.10^{-4}$ & $1,07.10^{-5}$ \\
\hline
\end{tabular}

Fonte: Elaborada pelo autor.

Nota-se dos resultados, considerando sinais individuais, que os problemas observados no primeiro teste para o LMS-NN tornam-se ainda mais evidentes quando a distribuição apresenta picos com espaçamentos consideráveis, além disso há também um crescimento no número de iterações e consequentemente aumento no tempo necessário para para convergência do algoritmo. 
Como era de se esperar, tendo em vista os resultados anteriores, a melhor resposta alcançada foi obtida com a análise considerando todo o conjunto de sinais.

\subsubsection{LMS-RNN}

Abaixo seguem os gráficos das distribuições alcançadas através do LMS-RNN para cada um dos seis sinais de decaimento simulados conforme descrito no inicio da sessão e da análise considerando todo o conjunto de sinais. Os parâmetros utilizados nos testes com cada um dos sinais, além do erro médio quadrático da resposta em relação a distribuição simulada estão relacionados na tabela 3.7 .

Figura 3.35 - Distribuição obtida através do algoritmo LMS-RNN para o sinal simulado com $\tau^{*}=$ $30 \mu s$ e comparado à distribuição simulada do teste dois.

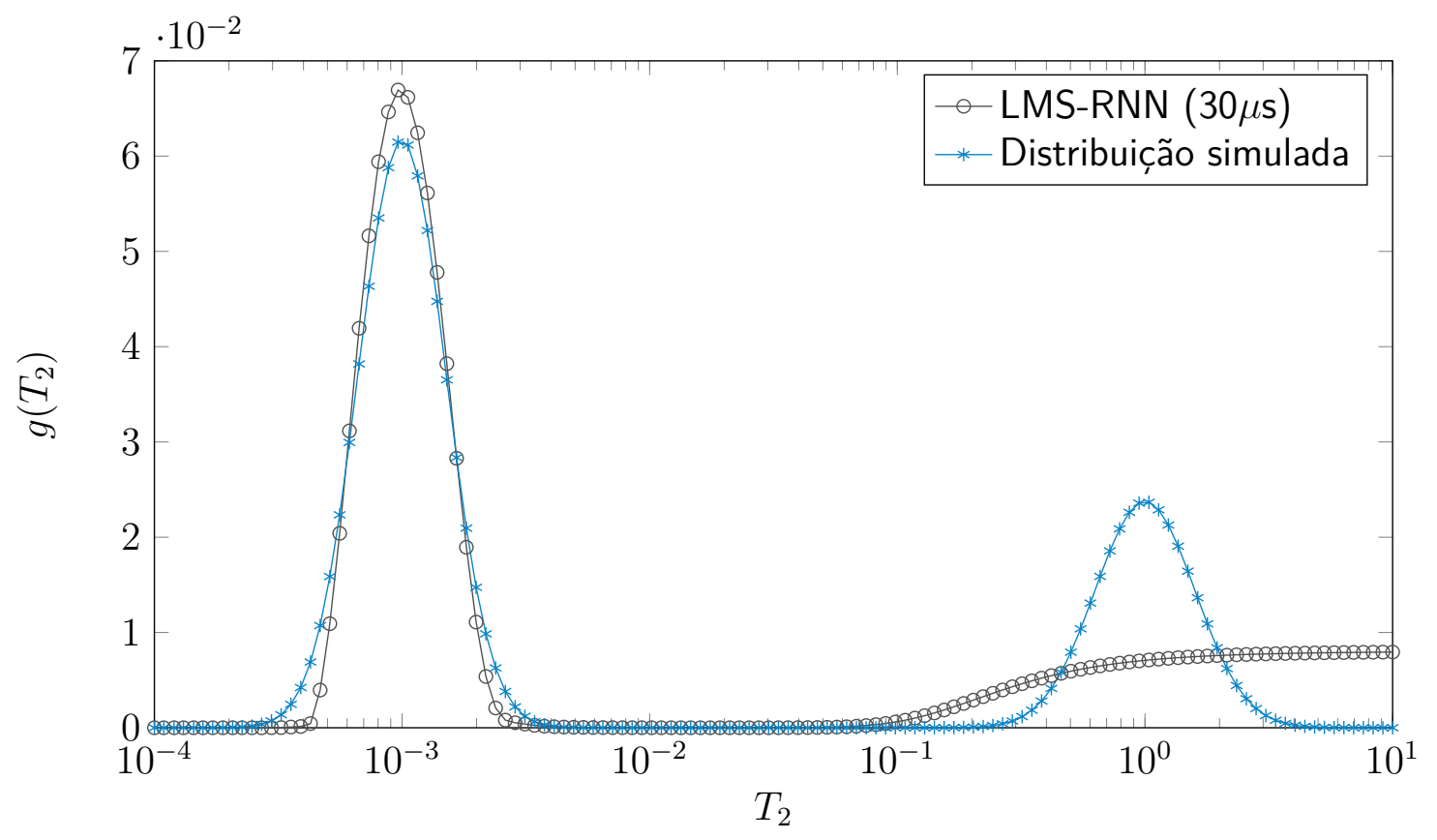

Fonte: Elaborada pelo autor. 
Figura 3.36 - Distribuição obtida através do algoritmo LMS-RNN para o sinal simulado com $\tau^{*}=$ $60 \mu s$ e comparado à distribuição simulada do teste dois.

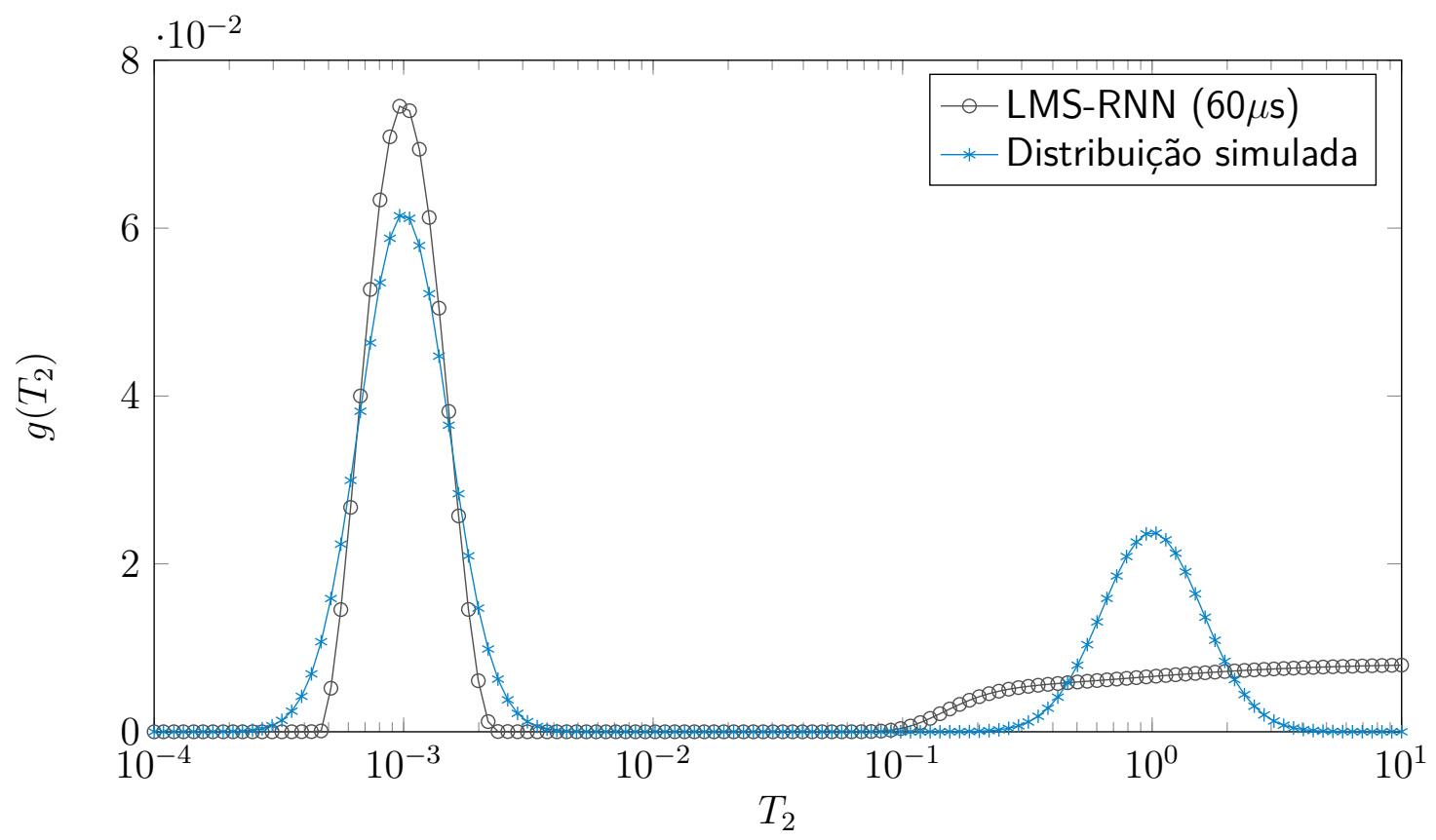

Fonte: Elaborada pelo autor.

Figura 3.37 - Distribuição obtida através do algoritmo LMS-RNN para o sinal simulado com $\tau^{*}=$ $120 \mu \mathrm{s}$ e comparado à distribuição simulada do teste dois.

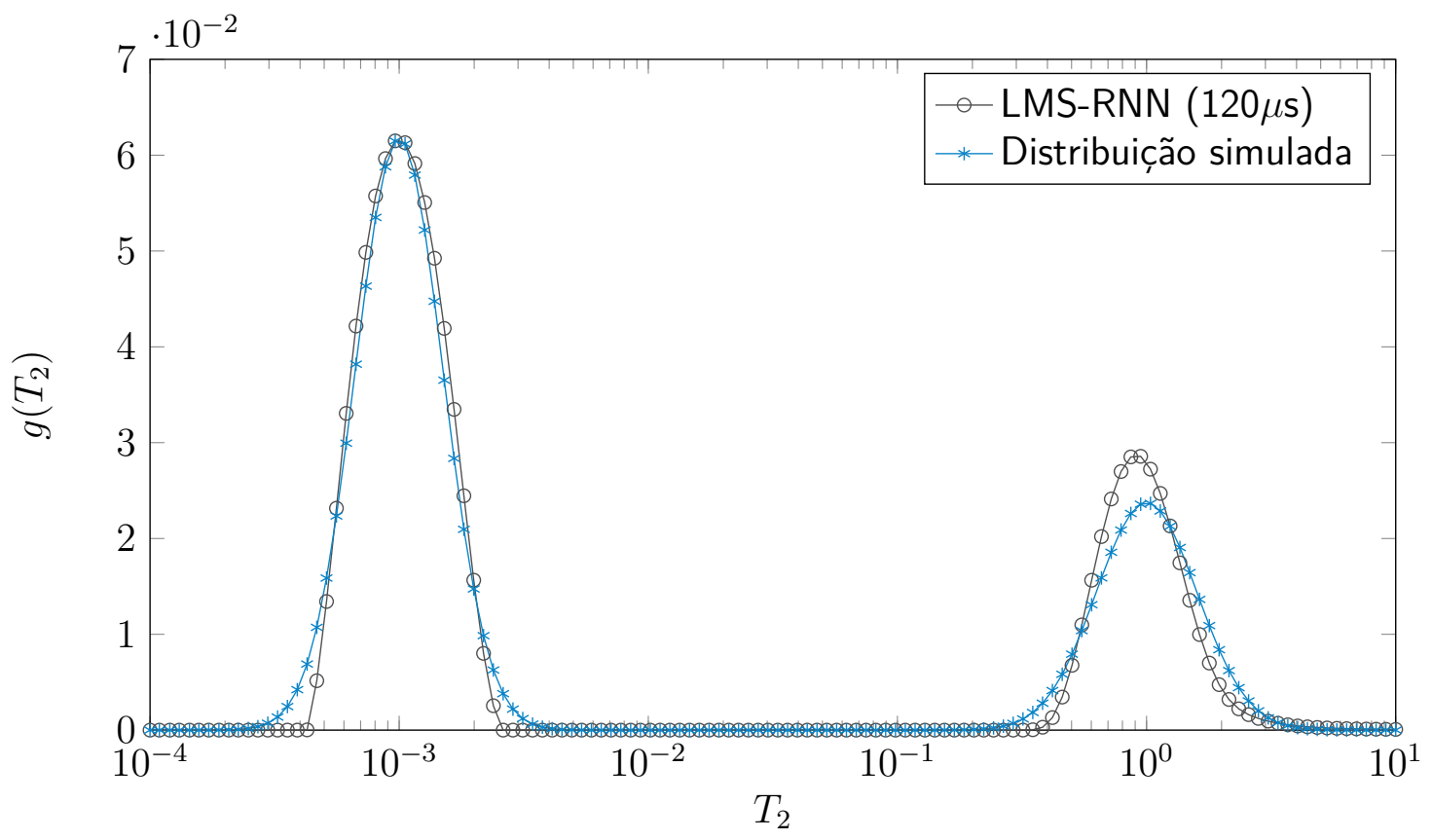

Fonte: Elaborada pelo autor. 
Figura 3.38 - Distribuição obtida através do algoritmo LMS-RNN para o sinal simulado $\operatorname{com} \tau^{*}=$ $240 \mu \mathrm{s}$ e comparado à distribuição simulada do teste dois.

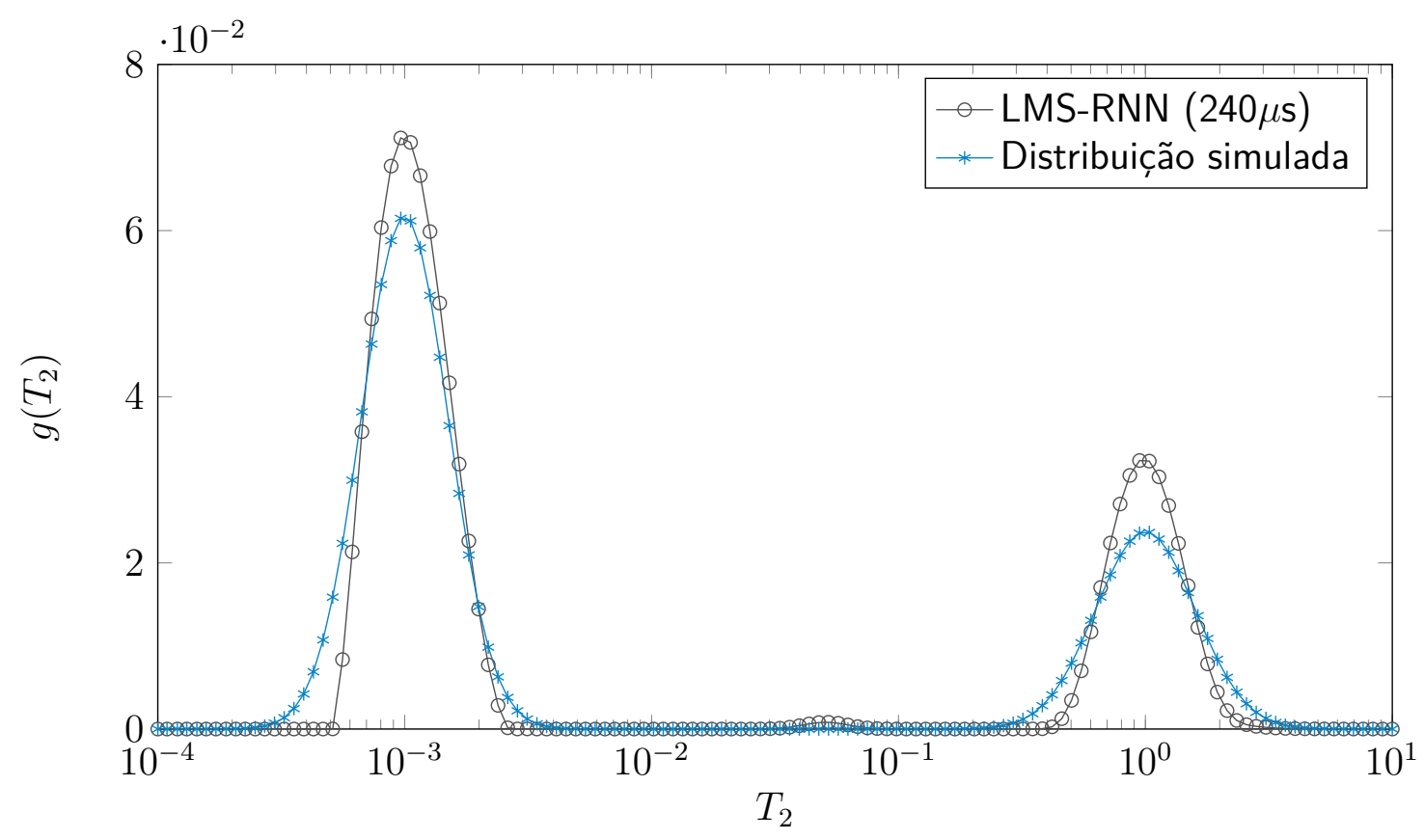

Fonte: Elaborada pelo autor.

Figura 3.39 - Distribuição obtida através do algoritmo LMS-RNN para o sinal simulado com $\tau^{*}=$ $480 \mu s$ e comparado à distribuição simulada do teste dois.

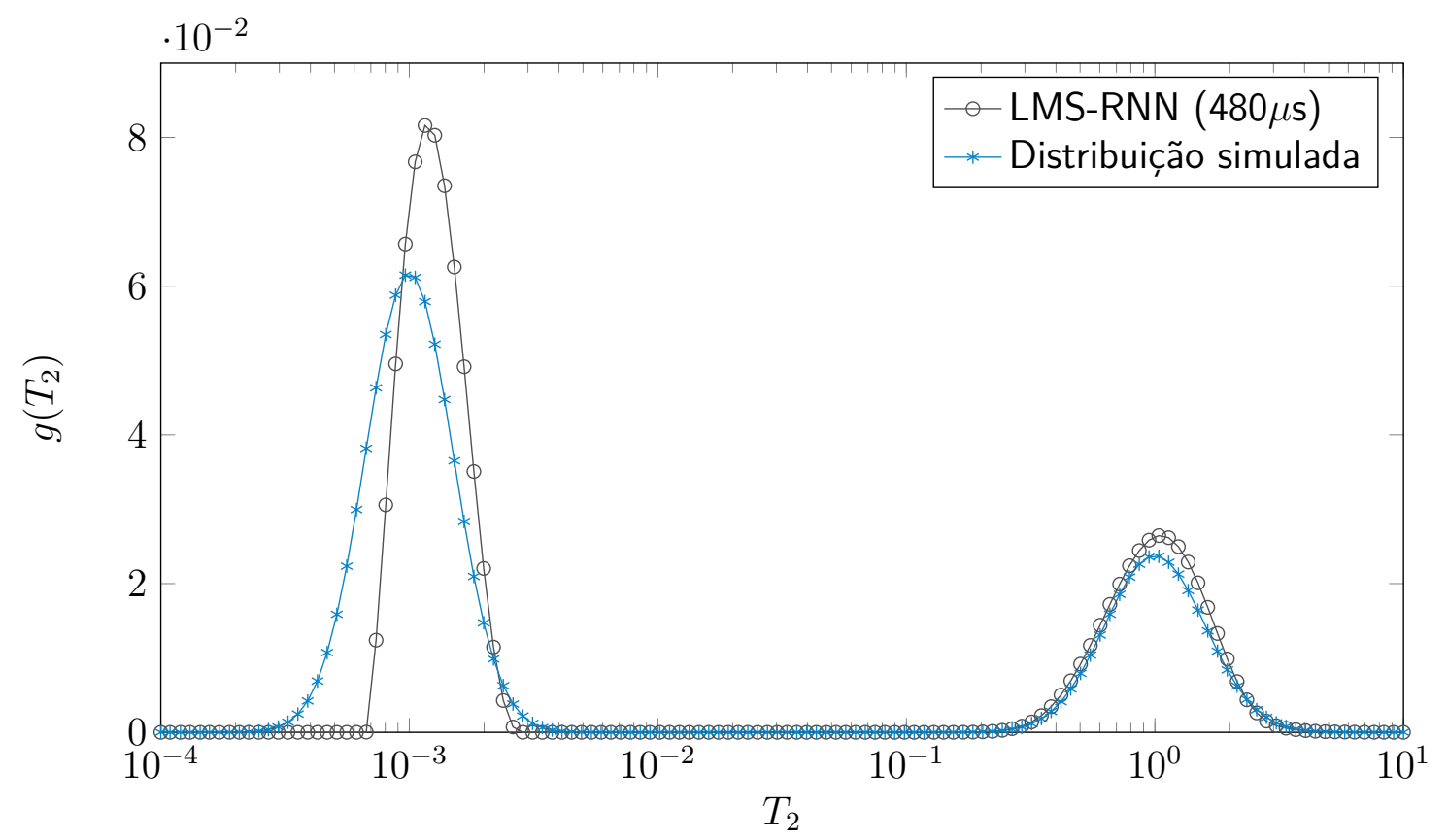

Fonte: Elaborada pelo autor. 
Figura 3.40 - Distribuição obtida através do algoritmo LMS-RNN para o sinal simulado com $\tau^{*}=$ $960 \mu \mathrm{s}$ e comparado à distribuição simulada do teste dois.

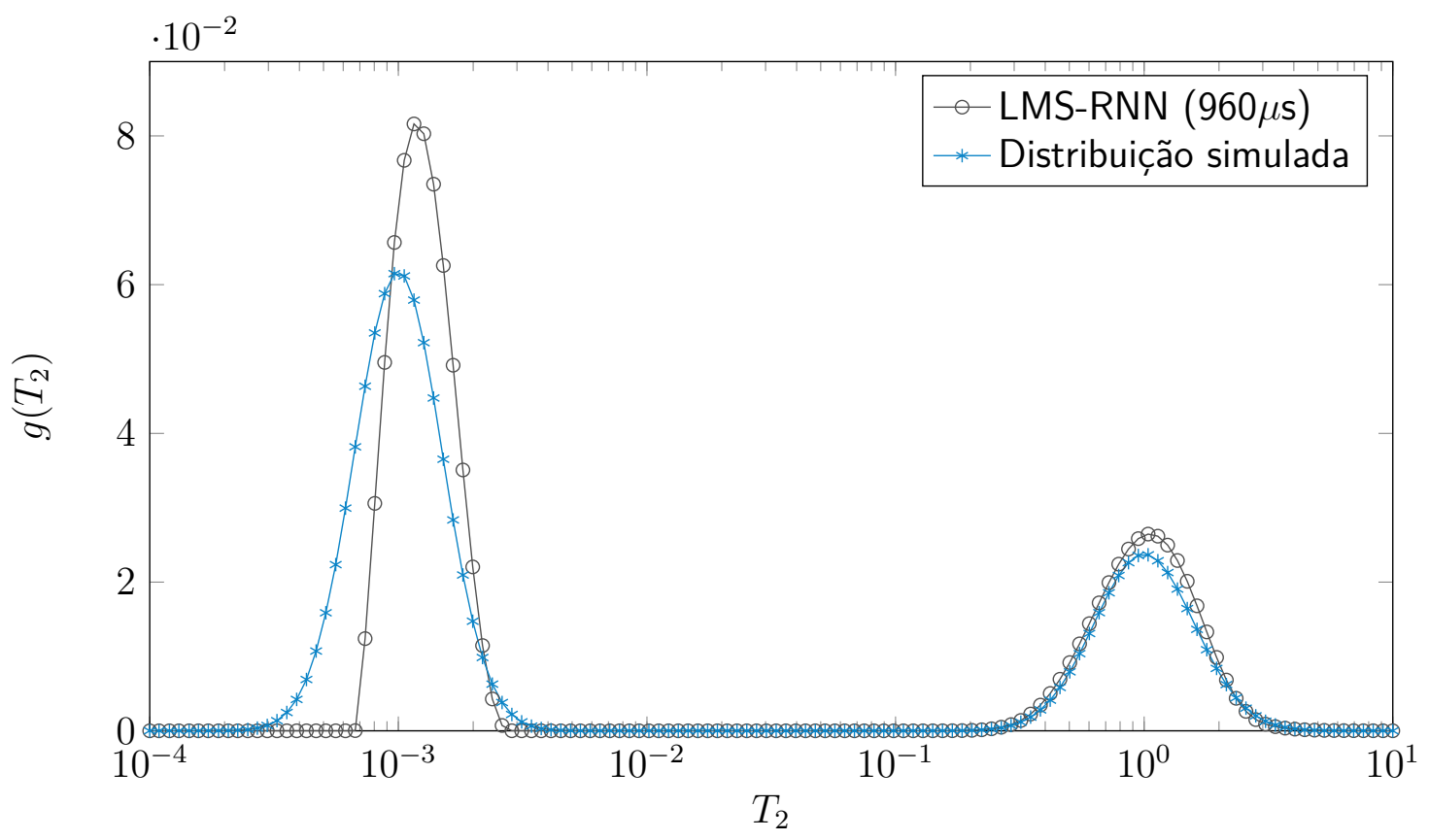

Fonte: Elaborada pelo autor. 
Figura 3.41 - Distribuição obtida através do algoritmo LMS-RNN combinando os sinais simulados com $\tau^{*}=30 \mu s, 60 \mu s, 120 \mu s, 240 \mu s, 480 \mu s$ e $960 \mu s$ e comparado à distribuição simulada.

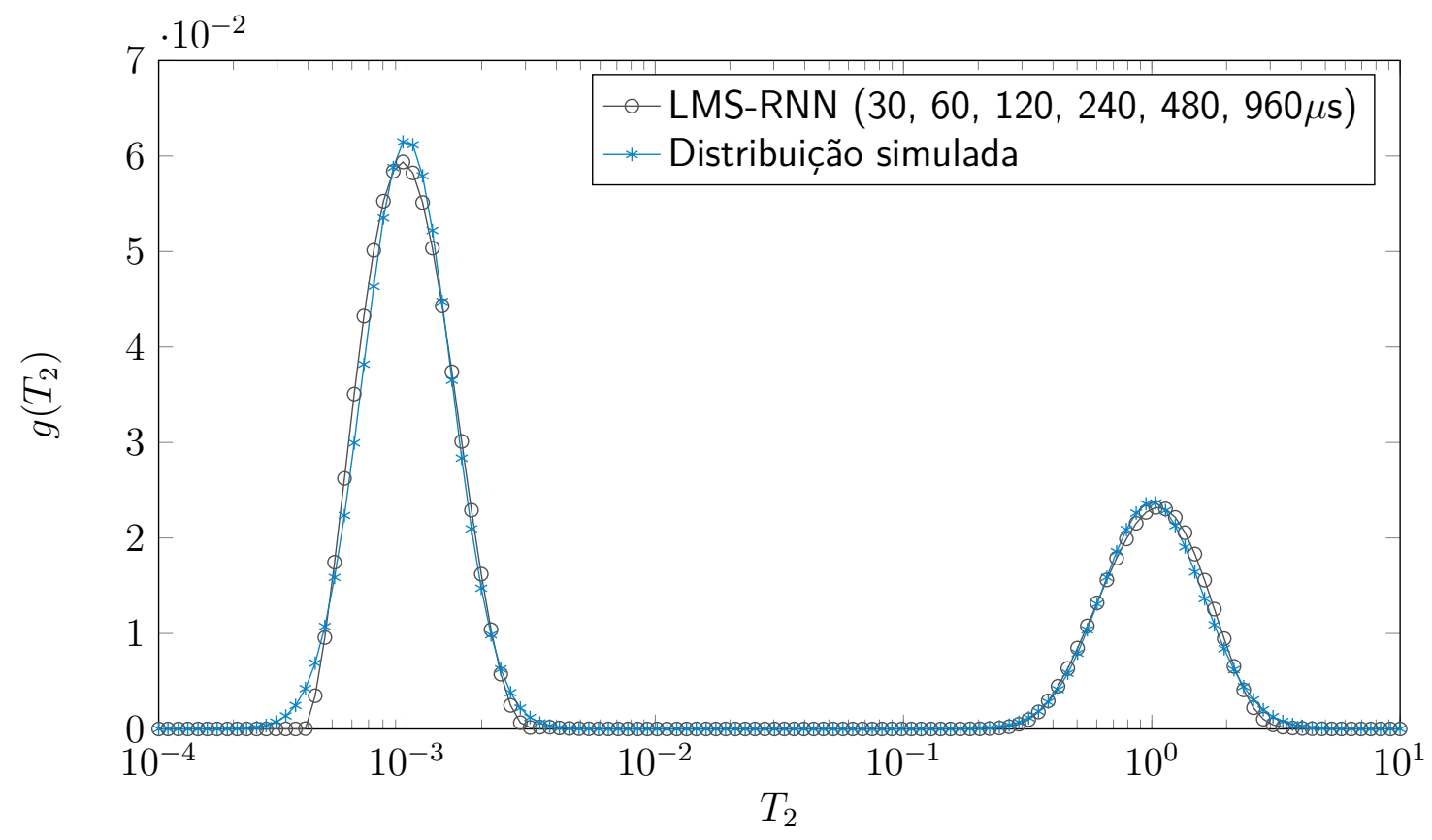

Fonte: Elaborada pelo autor.

Tabela 3.7 - Parâmetros utilizados e resultados obtidos com algoritmos LMS-RNN.

\begin{tabular}{ccccccc}
$\tau^{*}$ & $\eta$ & $\lambda$ & iterações & tempo(s) & convergência média & MSE \\
\hline \hline $30 \mu s$ & $10^{-5}$ & $5.10^{-4}$ & 6902 & 316 & $5.10^{-4}$ & $2,67.10^{-5}$ \\
\hline $60 \mu s$ & $10^{-5}$ & $5.10^{-4}$ & 8705 & 326 & $5.10^{-4}$ & $3,65.10^{-5}$ \\
\hline $120 \mu s$ & $10^{-5}$ & $5.10^{-4}$ & 20335 & 1192 & $5.10^{-4}$ & $4,36.10^{-6}$ \\
\hline $240 \mu s$ & $10^{-5}$ & $5.10^{-4}$ & 16961 & 1130 & $5.10^{-4}$ & $1,41.10^{-5}$ \\
\hline $480 \mu s$ & $10^{-5}$ & $5.10^{-4}$ & 18876 & 1202 & $5.10^{-4}$ & $7,02.10^{-5}$ \\
\hline $960 \mu s$ & $10^{-5}$ & $10^{-2}$ & 100000 & 2150 & $5.10^{-4}$ & $3,75.10^{-5}$ \\
\hline $30 \mu s, 60 \mu s, 120 \mu s$, & & & & & & \\
$240 \mu s, 480 \mu s, 960 \mu s$ & $10^{-5}$ & $5.10^{-4}$ & 4180 & 619 & $5.10^{-4}$ & $1,47.10^{-6}$ \\
\hline
\end{tabular}

Fonte: Elaborada pelo autor.

Diferente do primeiro teste, nota-se que o acréscimo do termo de regularização no LMSNN não foi suficiente para contornar as dificuldades envolvendo análise de sinais individuais formados a partir de distribuições muito espaçadas. Novamente o melhor resultado está associado a análise considerando todo o conjunto de sinais. 


\subsubsection{Hopfield}

Abaixo seguem os gráficos das distribuições alcançadas através da rede recorrente de Hopfield para cada um dos seis sinais de decaimento simulados conforme descrito no inicio da sessão. Os parâmetros utilizados nos testes com cada um dos sinais, além do erro médio quadrático da resposta em relação a distribuição simulada estão relacionados na tabela 3.8.

Figura 3.42 - Distribuição obtida através do algoritmo da rede recorrente de Hopfield para o sinal simulado com $\tau^{*}=30 \mu \mathrm{s}$ e comparado à distribuição simulada do teste dois.

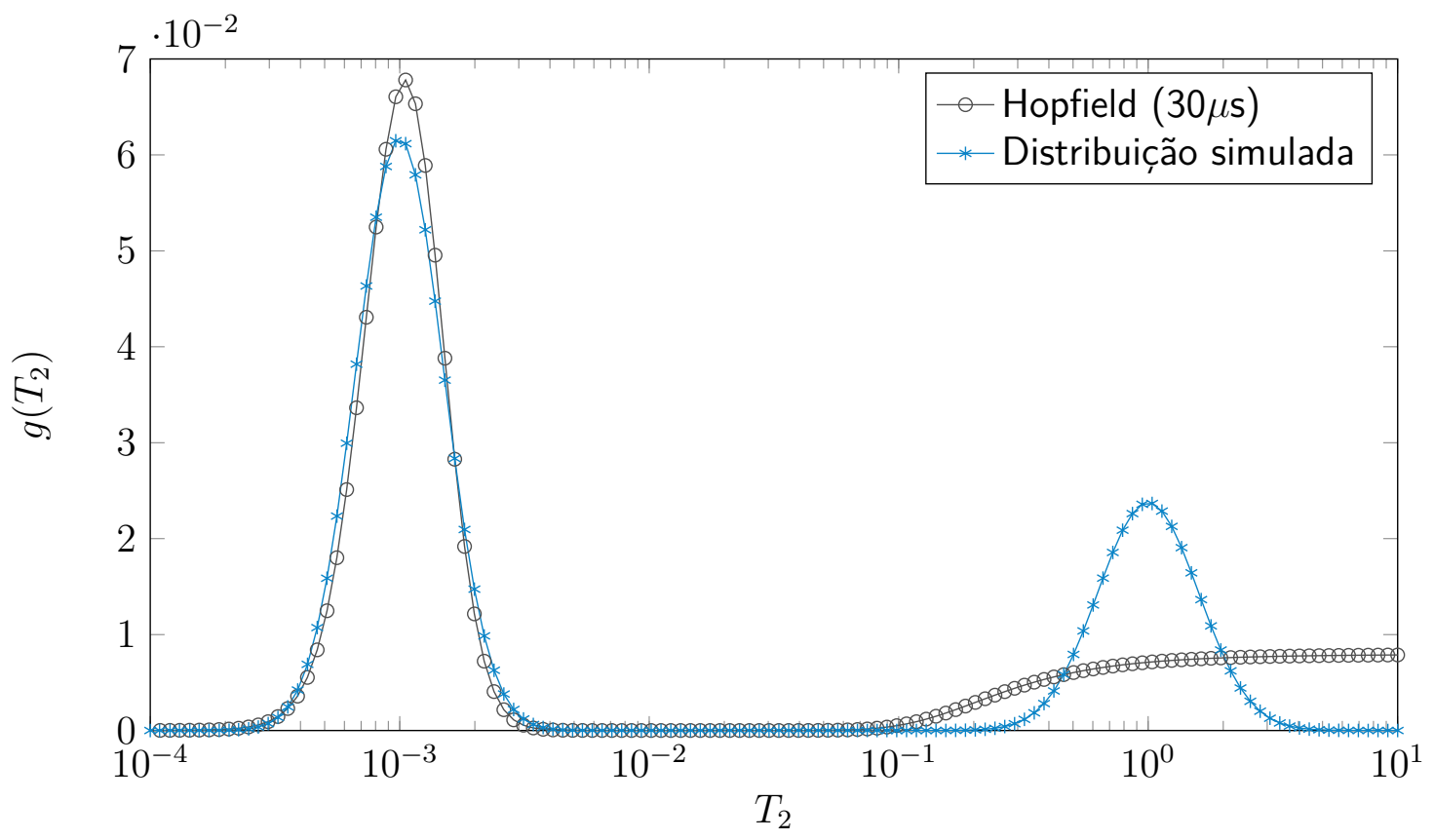

Fonte: Elaborada pelo autor. 
Figura 3.43 - Distribuição obtida através do algoritmo da rede recorrente de Hopfield para o sinal simulado com $\tau^{*}=60 \mu \mathrm{s}$ e comparado à distribuição simulada do teste dois.

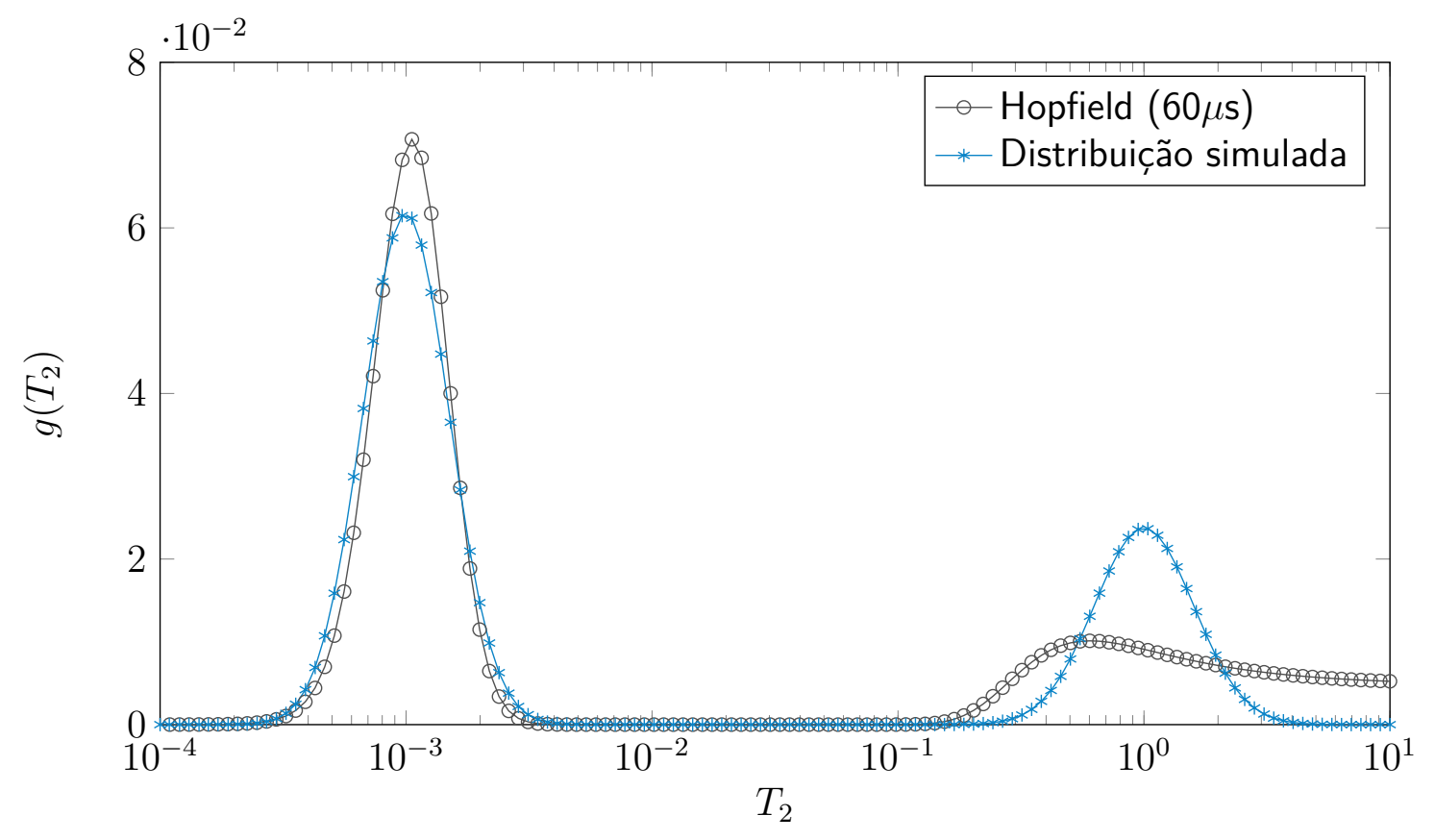

Fonte: Elaborada pelo autor.

Figura 3.44 - Distribuição obtida através do algoritmo da rede recorrente de Hopfield para o sinal simulado com $\tau^{*}=120 \mu \mathrm{s}$ e comparado à distribuição simulada do teste dois.

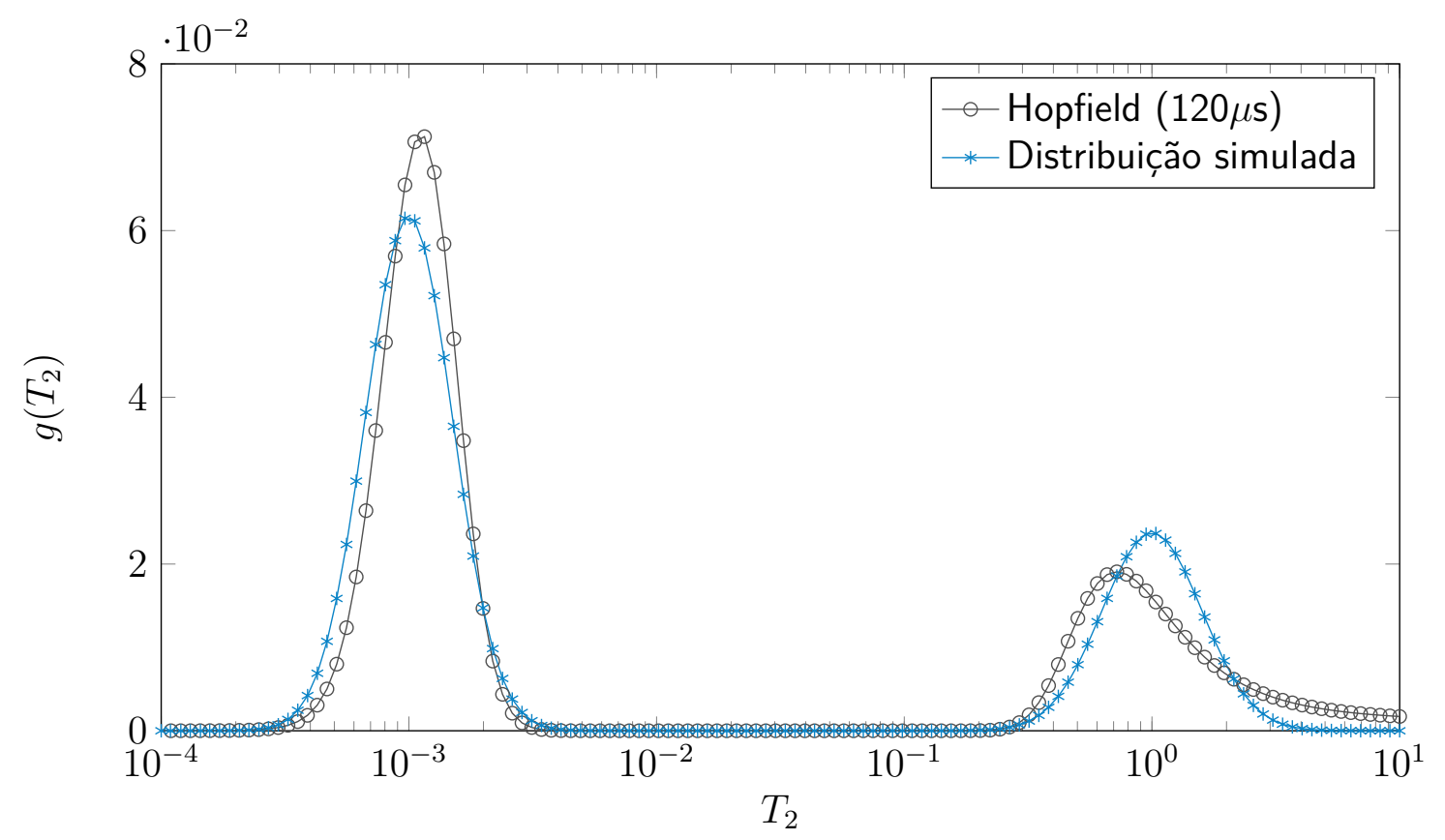

Fonte: Elaborada pelo autor. 
Figura 3.45 - Distribuição obtida através do algoritmo da rede recorrente de Hopfield para o sinal simulado com $\tau^{*}=240 \mu \mathrm{s}$ e comparado à distribuição simulada do teste dois.

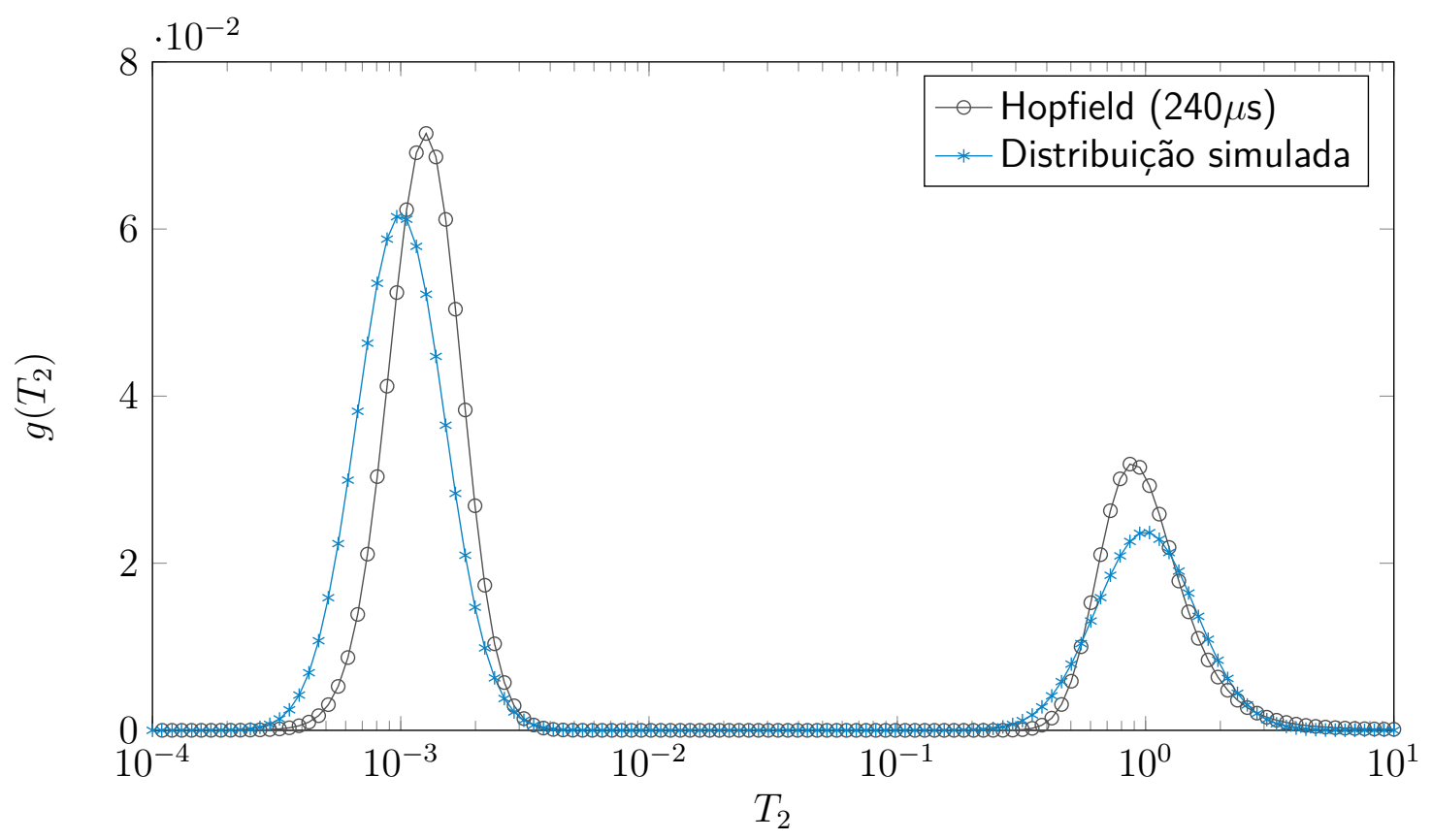

Fonte: Elaborada pelo autor.

Figura 3.46 - Distribuição obtida através do algoritmo da rede recorrente de Hopfield para o sinal simulado com $\tau^{*}=480 \mu \mathrm{s}$ e comparado à distribuição simulada do teste dois.

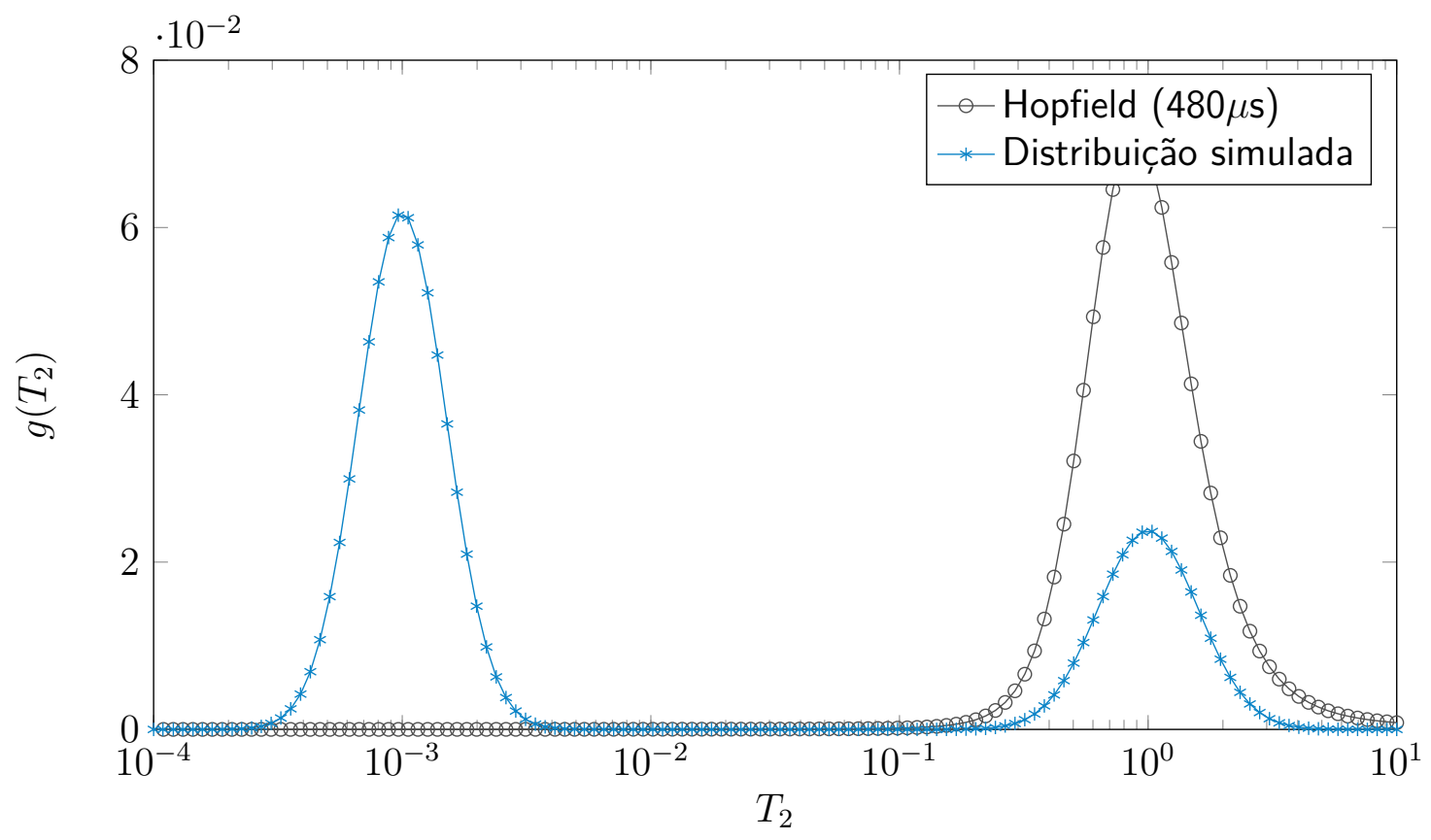

Fonte: Elaborada pelo autor. 
Figura 3.47 - Distribuição obtida através do algoritmo da rede recorrente de Hopfield para o sinal simulado com $\tau^{*}=960 \mu \mathrm{s}$ e comparado à distribuição simulada do teste dois.

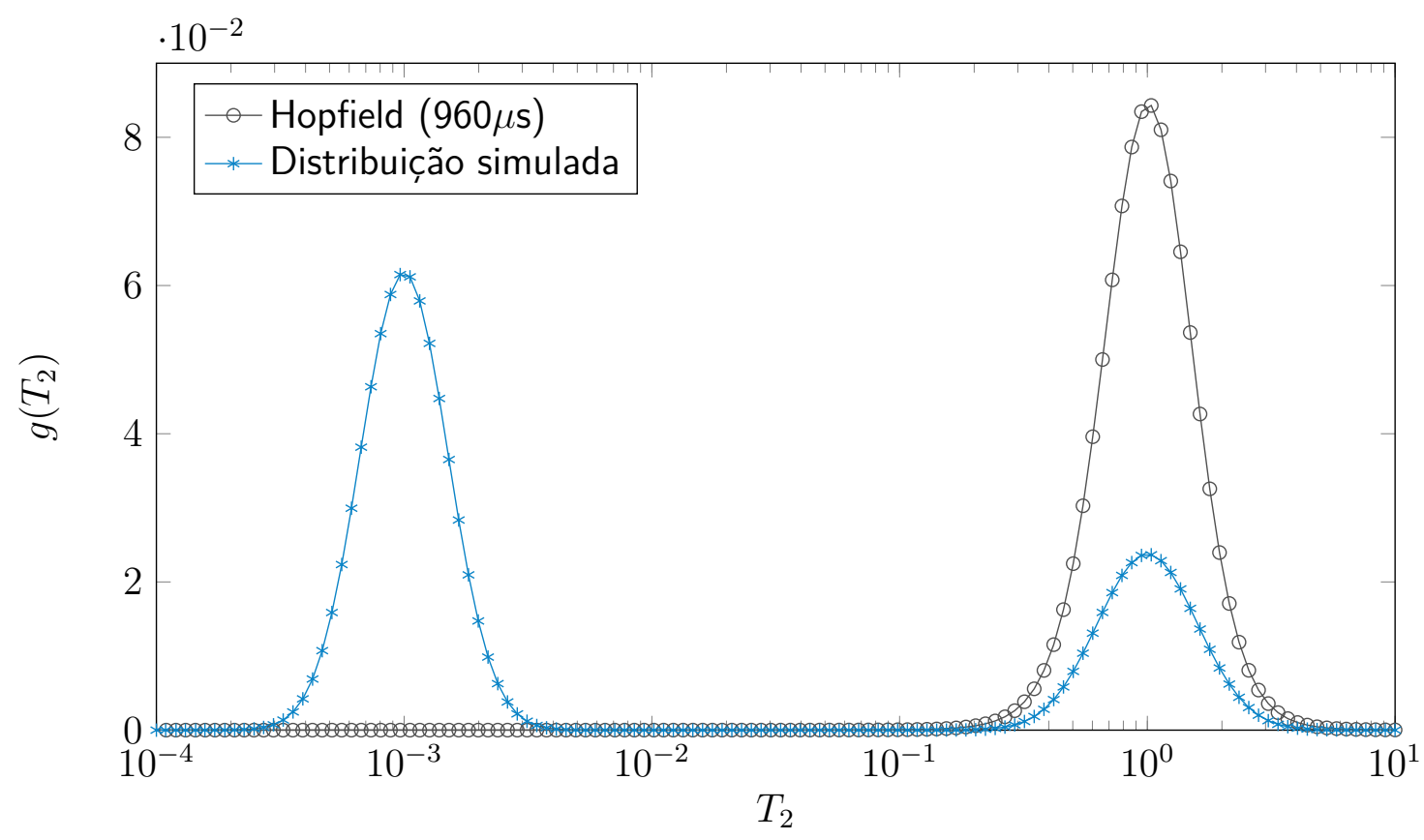

Fonte: Elaborada pelo autor.

Tabela 3.8 - Parâmetros utilizados e resultados obtidos com algoritmo da rede recorrente de Hopfield para o teste dois.

\begin{tabular}{cccccc}
$\tau^{*}$ & $h$ & iterações & tempo(s) & convergência média & MSE \\
\hline \hline $30 \mu s$ & $1,5.10^{-3}$ & 8840 & 87 & $10^{-4}$ & $2,57.10^{-5}$ \\
\hline $60 \mu s$ & $1,5.10^{-3}$ & 14335 & 209 & $10^{-4}$ & $2,13.10^{-5}$ \\
\hline $120 \mu s$ & $1,5.10^{-3}$ & 23420 & 317 & $10^{-4}$ & $1,69.10^{-5}$ \\
\hline $240 \mu s$ & $1,5.10^{-3}$ & 34494 & 361 & $10^{-4}$ & $1,20.10^{-5}$ \\
\hline $480 \mu s$ & $1,5.10^{-3}$ & 1407 & 15 & $10^{-4}$ & $4,09.10^{-4}$ \\
\hline $960 \mu s$ & $1,5.10^{-3}$ & 1049 & 11 & $10^{-4}$ & $4,61.10^{-4}$ \\
\hline
\end{tabular}

Fonte: Elaborada pelo autor.

Dentre os testes com a distribuição espaçada a rede recorrente de Hopfield foi o algoritmo que demonstrou o pior comportamento. Conforme $\tau^{*}$ vai assumindo diferentes valores, regiões da distribuição vão sendo totalmente ignoradas, conforme observa-se principalmente nas figuras $3.43,3.44,3.46$ e 3.47 . 


\subsubsection{LS-R (SVD)}

Abaixo seguem os resultados obtidos com o algoritmo dos mínimos quadrados regularizado para o teste dois. A tabela 3.9 contém os parâmetros utilizados na análise com cada um dos sinais de decaimento simulados, além do erro médio quadrático da resposta em relação a distribuição simulada. As figuras 3.48, 3.49, 3.50, 3.51, 3.52 e 3.53 comparam os resultados obtidos com algoritmo em relação a distribuição simulada.

Figura 3.48 - Distribuição obtida através do algoritmo LS-R para o sinal simulado com $\tau^{*}=30 \mu \mathrm{s}$ e comparado à distribuição simulada do teste dois.

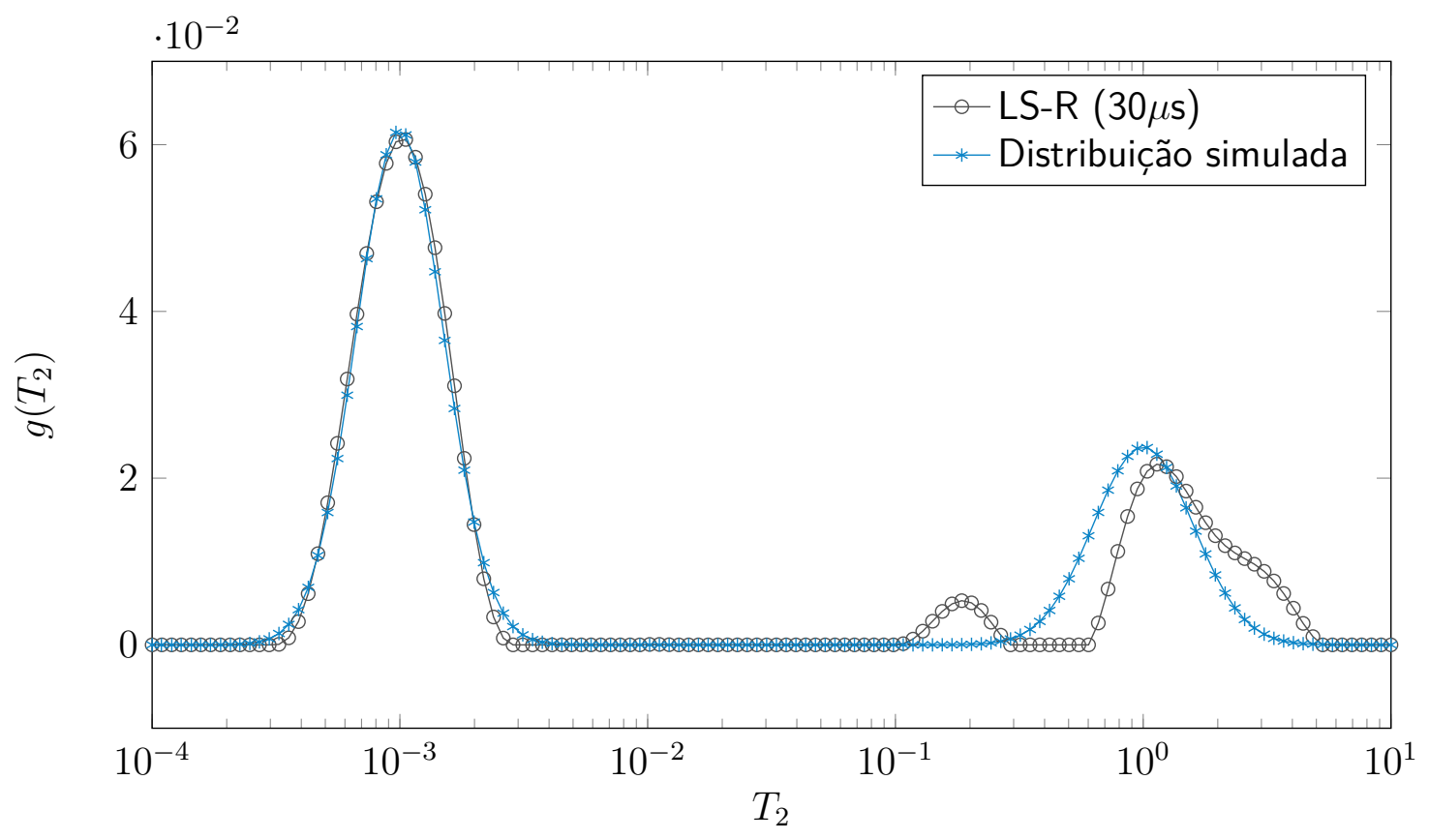

Fonte: Elaborada pelo autor. 
Figura 3.49 - Distribuição obtida através do algoritmo LS-R para o sinal simulado com $\tau^{*}=60 \mu \mathrm{s}$ e comparado à distribuição simulada do teste dois.

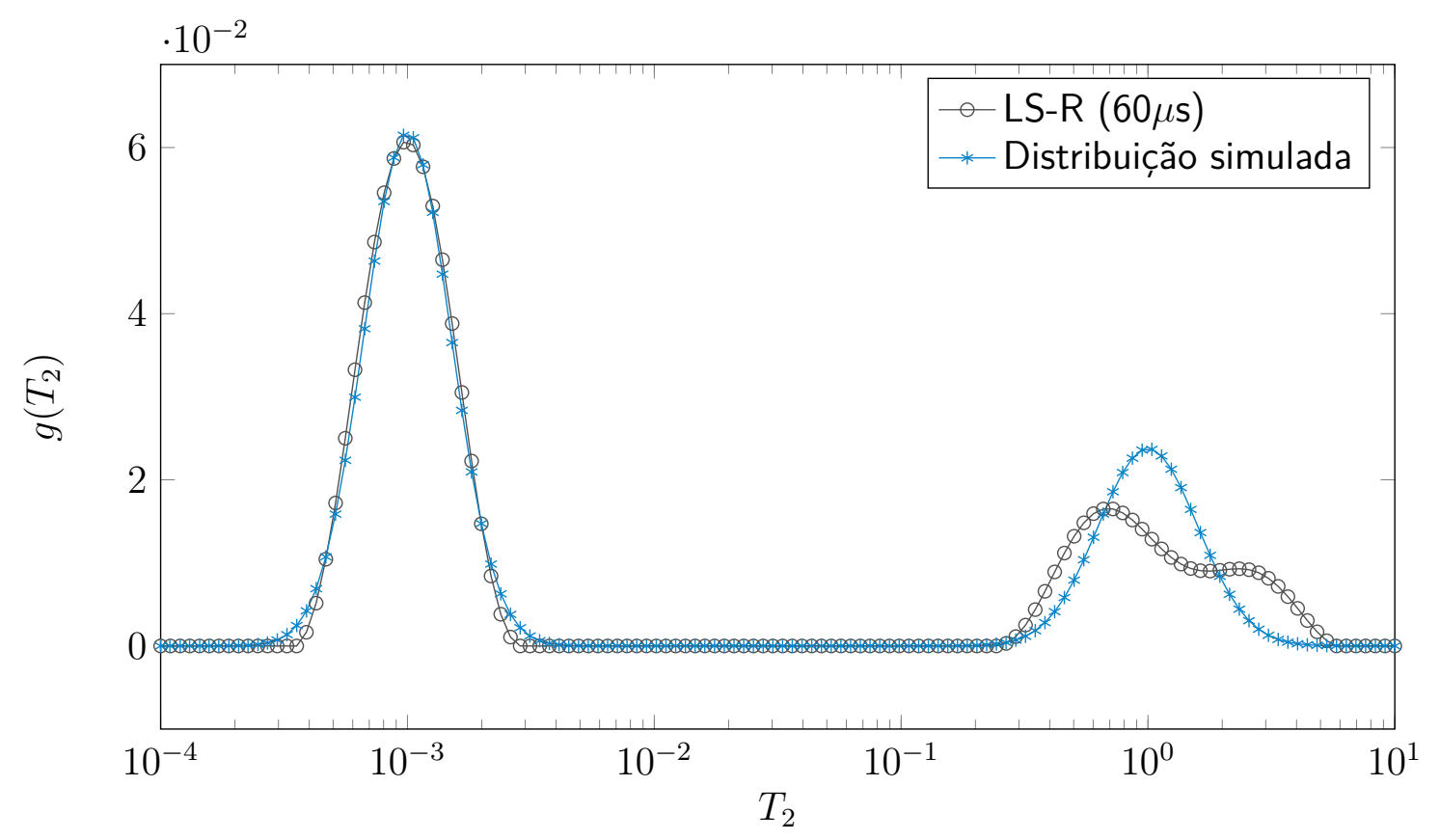

Fonte: Elaborada pelo autor.

Figura 3.50 - Distribuição obtida através do algoritmo LS-R para o sinal simulado com $\tau^{*}=120 \mu \mathrm{s}$ e comparado à distribuição simulada do teste dois.

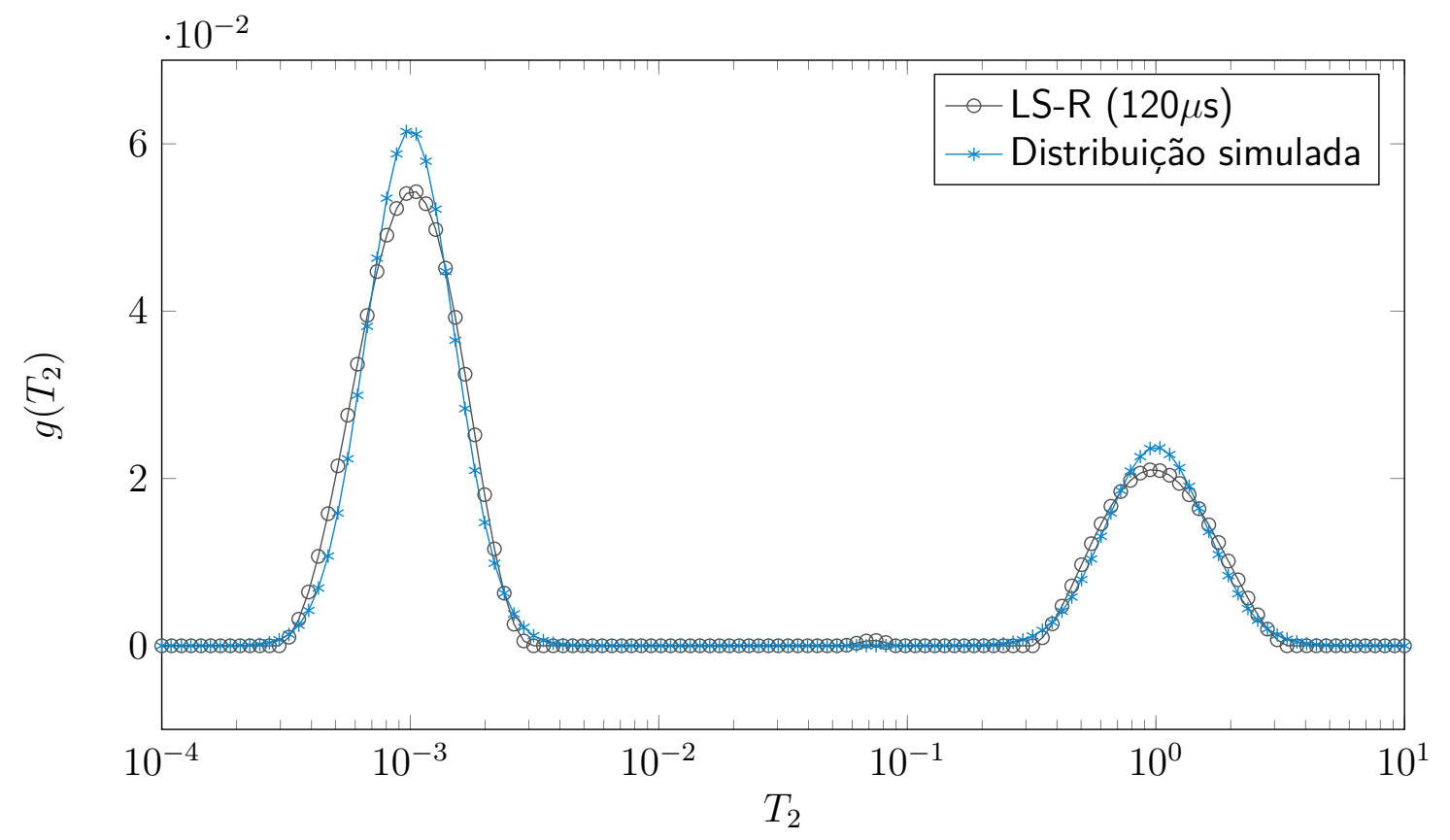

Fonte: Elaborada pelo autor. 
Figura 3.51 - Distribuição obtida através do algoritmo LS-R para o sinal simulado com $\tau^{*}=240 \mu s$ e comparado à distribuição simulada do teste dois.

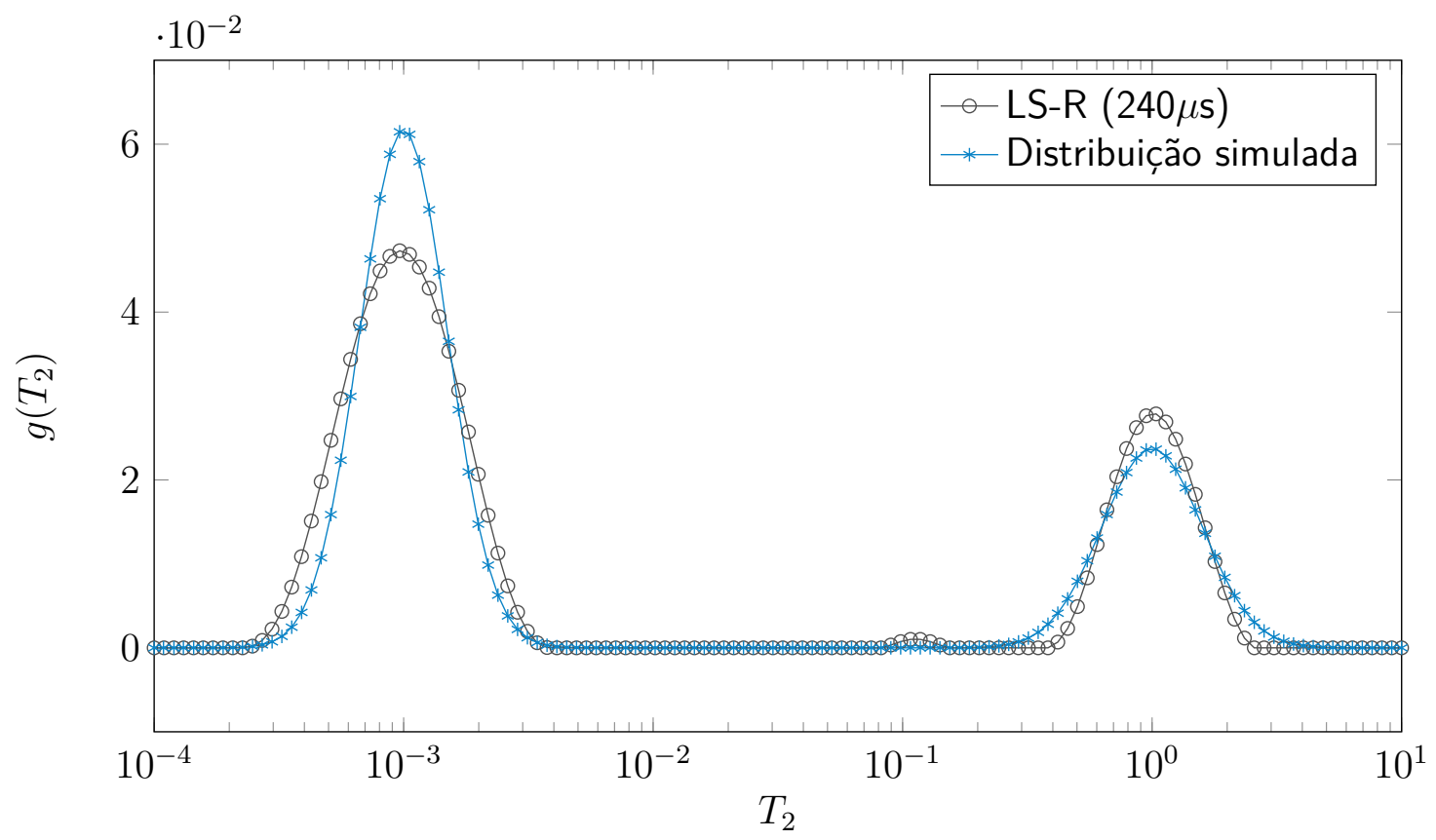

Fonte: Elaborada pelo autor.

Figura 3.52 - Distribuição obtida através do algoritmo LS-R para o sinal simulado com $\tau^{*}=480 \mu \mathrm{s}$ e comparado à distribuição simulada do teste dois.

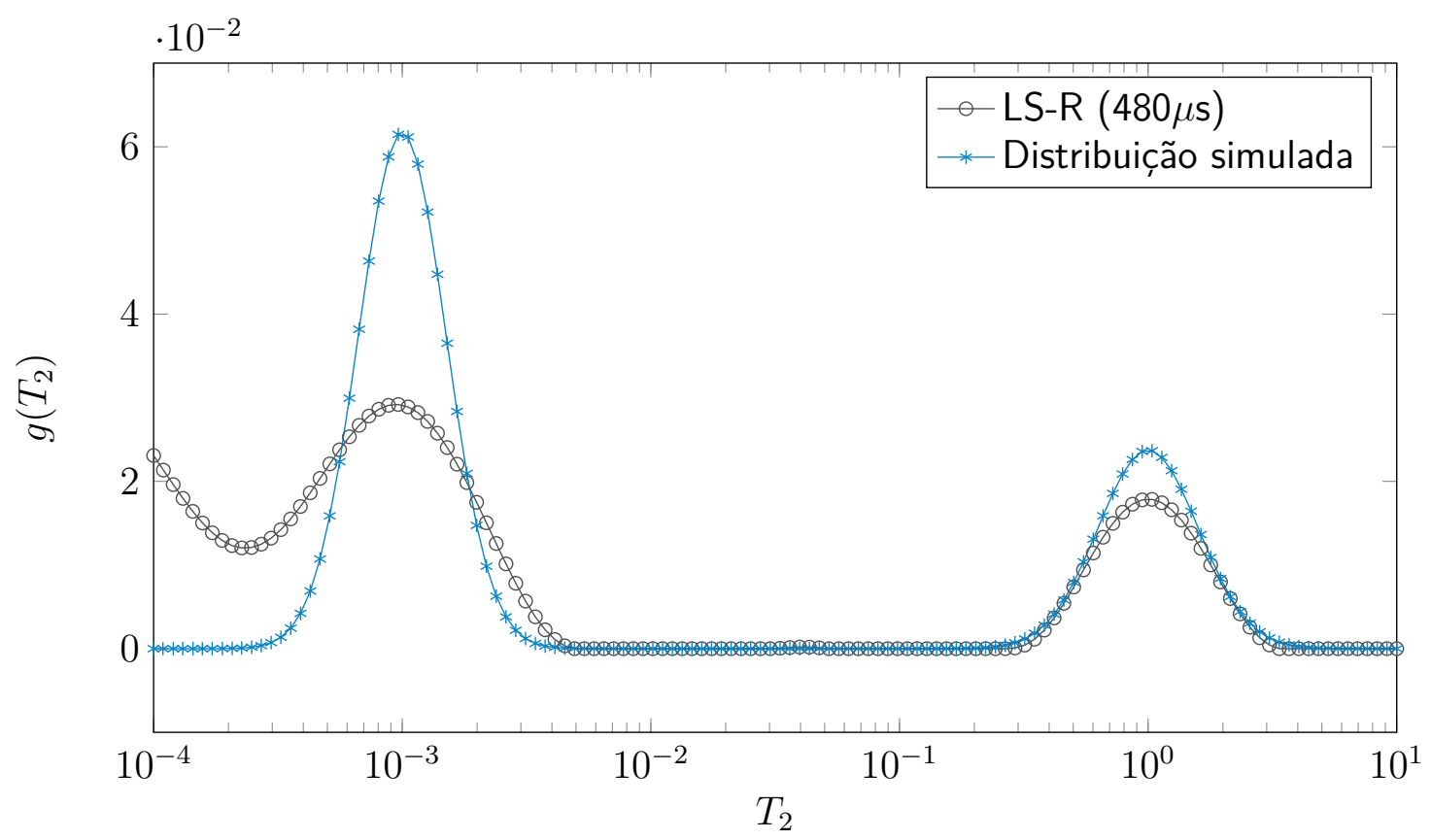

Fonte: Elaborada pelo autor. 
Figura 3.53 - Distribuição obtida através do algoritmo LS-R para o sinal simulado com $\tau^{*}=960 \mu s$ e comparado à distribuição simulada do teste dois.

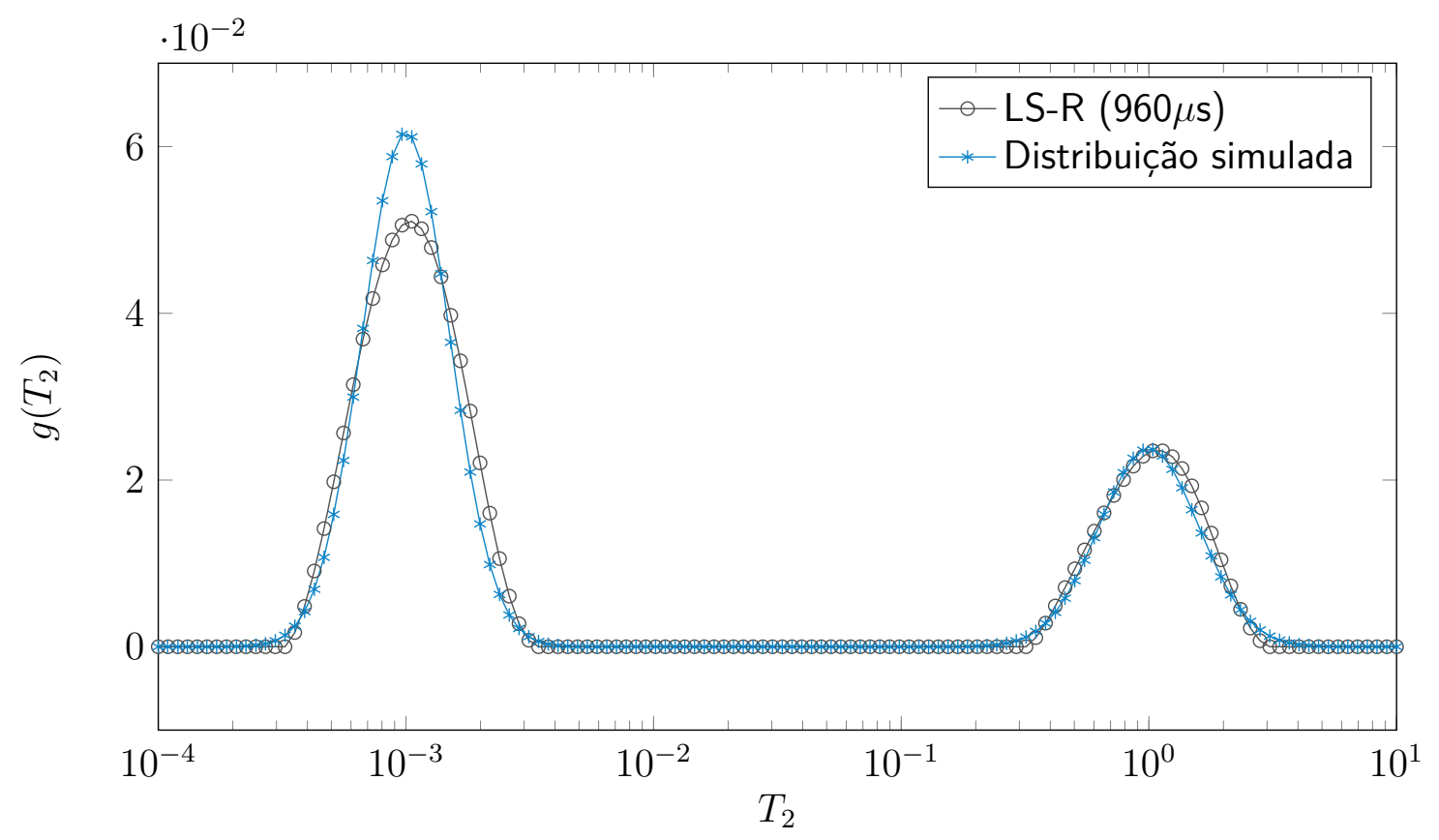

Fonte: Elaborada pelo autor.

Tabela 3.9 - Parâmetros utilizados e resultados obtidos com algoritmos LS-R.

\begin{tabular}{cccccc}
$\tau^{*}$ & $\lambda$ & iterações & tempo(s) & MSE & $\sum\left(g\left(T_{2}\right)<0\right)^{2}$ \\
\hline \hline $30 \mu s$ & 10 & 5 & 1 & $1,18.10^{-5}$ & $9,75.10^{-6}$ \\
\hline $60 \mu s$ & 10 & 19 & 2 & $9,20.10^{-6}$ & $9,71.10^{-6}$ \\
\hline $120 \mu s$ & 10 & 11 & 1 & $3,43.10^{-6}$ & $9,24.10^{-6}$ \\
\hline $240 \mu s$ & 10 & 7 & 1 & $1,27.10^{-5}$ & $8,36.10^{-6}$ \\
\hline $480 \mu s$ & 10 & 4 & 2 & $8,29.10^{-5}$ & $4,51.10^{-6}$ \\
\hline $960 \mu s$ & 10 & 8 & 1 & $6,27.10^{-6}$ & $9,22.10^{-6}$ \\
\hline
\end{tabular}

Fonte: Elaborada pelo autor.

Apesar de apresentar algumas oscilações o LS-R apresentou resposta regular para a maioria dos sinais e convergência relativamente rápida considerando a energia máxima imposta das componentes negativas da distribuição. 
Abaixo segue quadro comparativo entre os melhores resultados (em relação ao erro médio quadrático) considerando os quatro algoritmos no segundo experimento:

Tabela 3.10 - Comparativo entre os melhores resultados obtidos com cada algoritmo para o teste dois.

\begin{tabular}{ccccc} 
algoritmo & MSE & iterações & tempo(s) & $\sum\left(g\left(T_{2}\right)<0\right)^{2}$ \\
\hline \hline LMS-NN (múltiplos sinais) & $1,07.10^{-5}$ & 5425 & 830 & 0 \\
\hline LMS-RNN (múltiplos sinais) & $1,47.10^{-6}$ & 4180 & 619 & 0 \\
\hline Hopfield $(240 \mu s)$ & $1,20.10^{-5}$ & 34494 & 301 & 0 \\
\hline LS-R $(120 \mu s)$ & $3,43.10^{-6}$ & 11 & 1 & $9,24.10^{-6}$ \\
\hline
\end{tabular}

Fonte: Elaborada pelo autor.

\subsection{Teste 3 - distribuição delta de Kronecker}

O terceiro teste foi realizado utilizando distribuição de valores $T_{2}$ simulada através da distribuição conhecida como delta de Kronecker, definida como:

$$
\delta(x-k)= \begin{cases}1, & \text { se } x=k \\ 0, & \text { se } x \neq 0\end{cases}
$$

Assim como nos testes anteriores o sinal de magnetização com $2^{12}$ amostras foi formado a partir da distribuição simulada composta por $2^{16}$ valores de $T_{2}$ logaritimicamente espaçados no intervalo de $10^{-4}$ à $10^{1}$ segundos.

Este teste tem como finalidade analisar o comportamento dos algoritmos em situações cuja distribuição apresente descontinuidade acentuada, outros testes poderiam envolver uma distribuição triangular, uniforme ou ainda do tipo rampa. 
Figura 3.54 - Distribuição de valores obtidas através do algoritmo LMS-NN para $\tau^{*}=15 \mu \mathrm{s}$ comparado com a distribuição simulada.

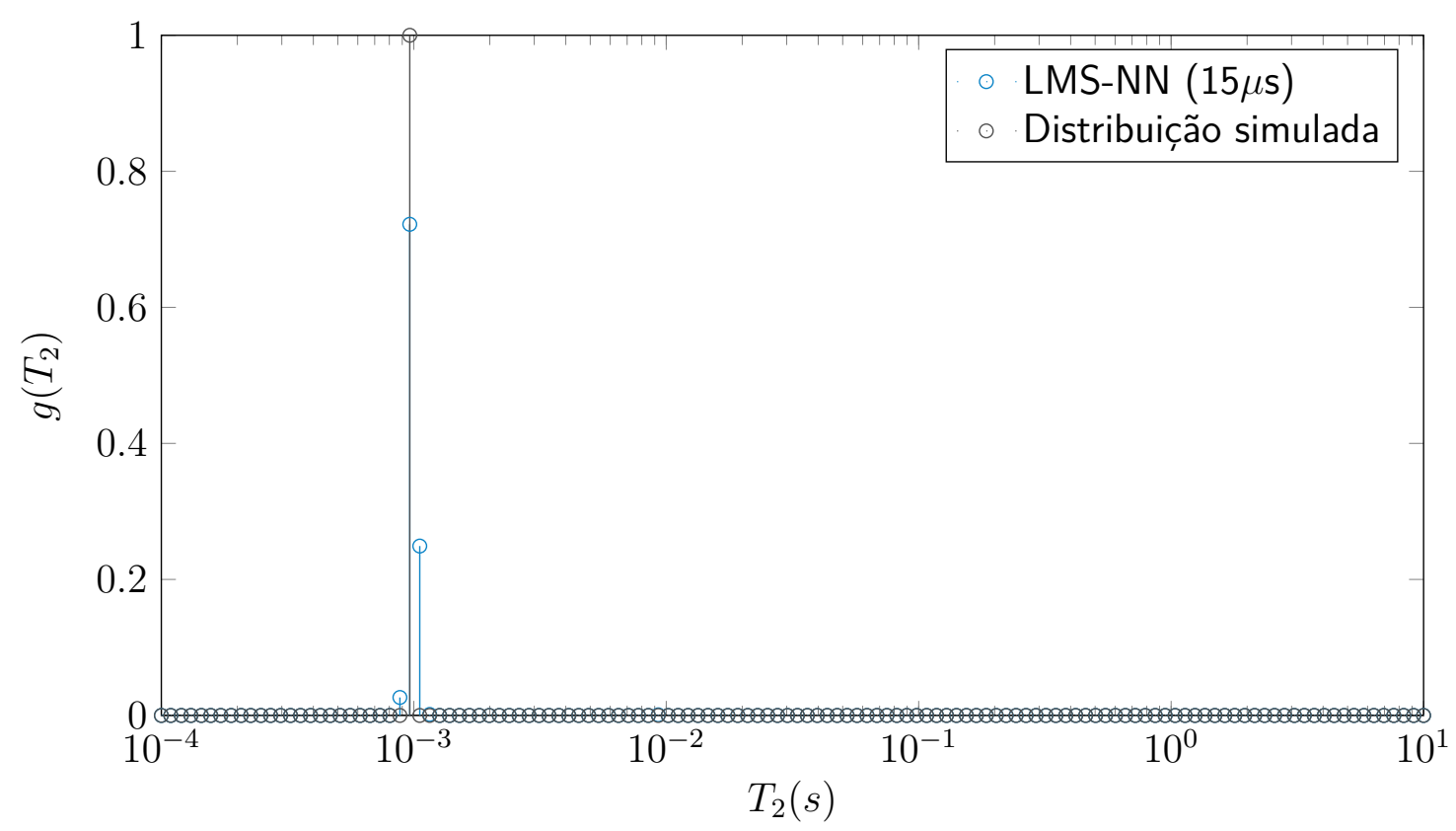

Fonte: Elaborada pelo autor.

Figura 3.55 - Distribuição de valores obtidas através do algoritmo LMS-RNN para $\tau^{*}=15 \mu \mathrm{s}$ comparado com a distribuição simulada.

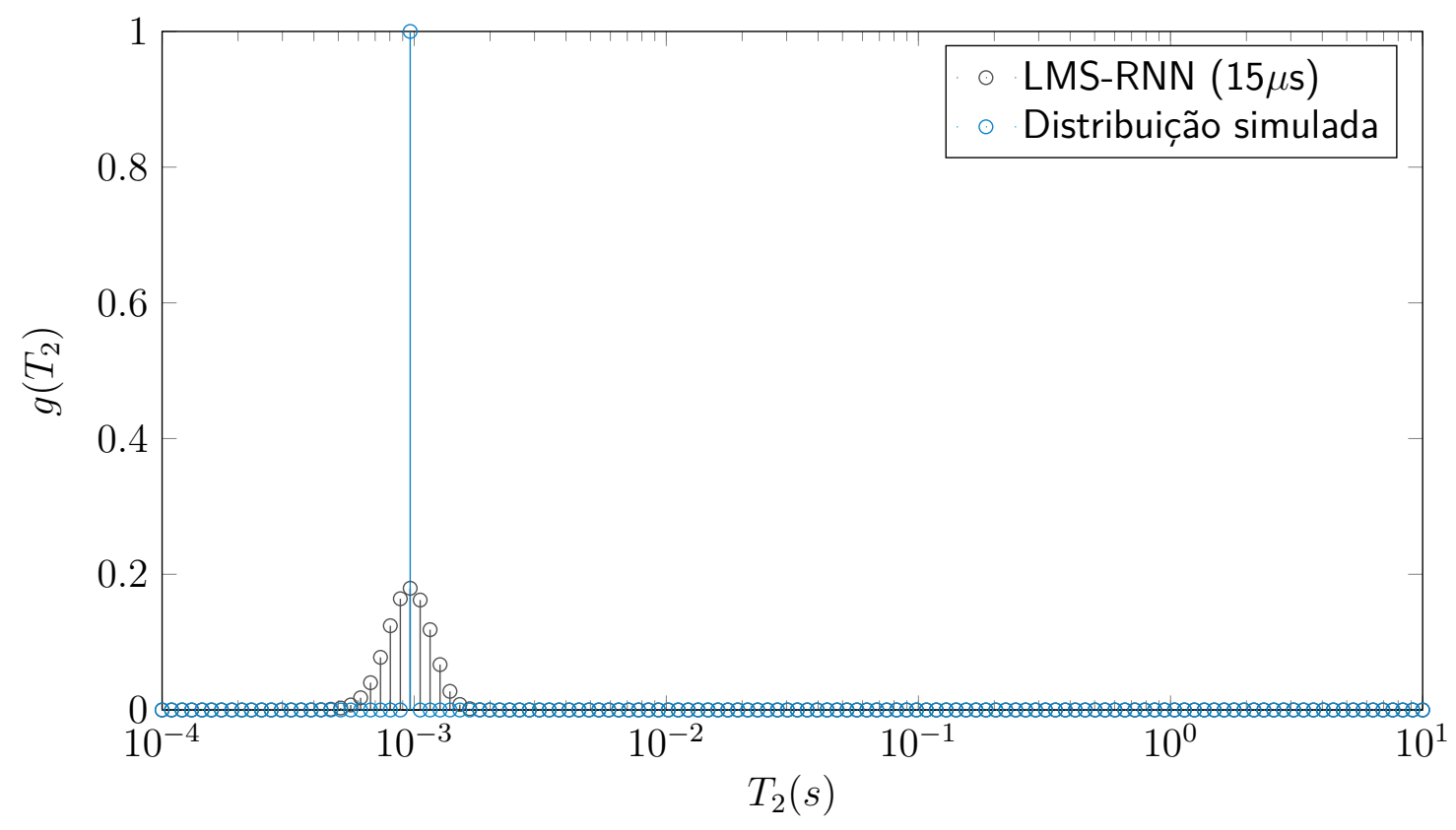

Fonte: Elaborada pelo autor. 
Figura 3.56 - Distribuição de valores obtidas através do algoritmo da rede recorrente de Hopfield para $\tau^{*}=15 \mu \mathrm{s}$ comparado com a distribuição simulada.

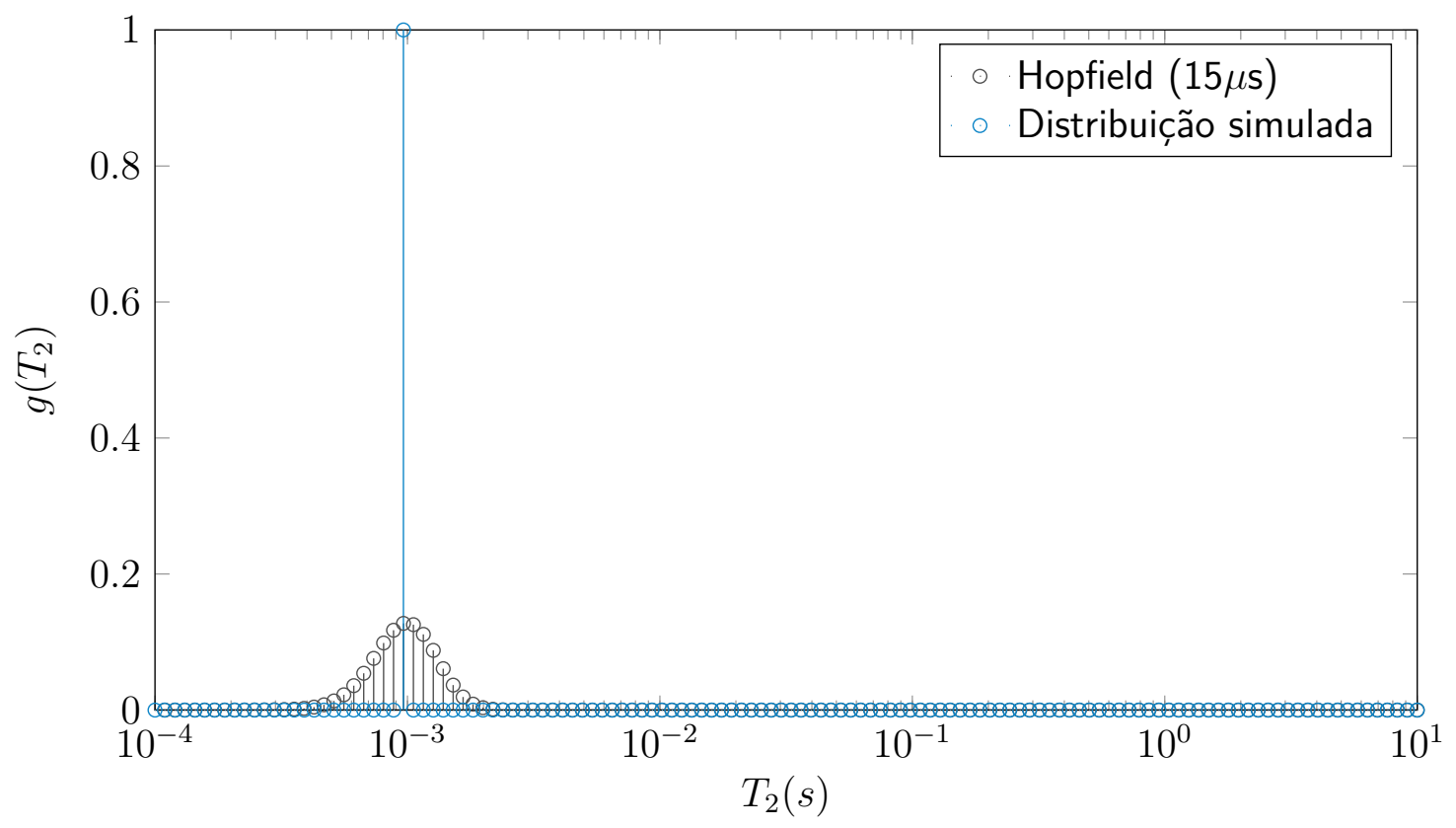

Fonte: Elaborada pelo autor. 
Figura 3.57 - Distribuição de valores obtidas através do algoritmo dos mínimos quadrados regularizado para $\tau^{*}=15 \mu \mathrm{s}$ comparado com a distribuição simulada.

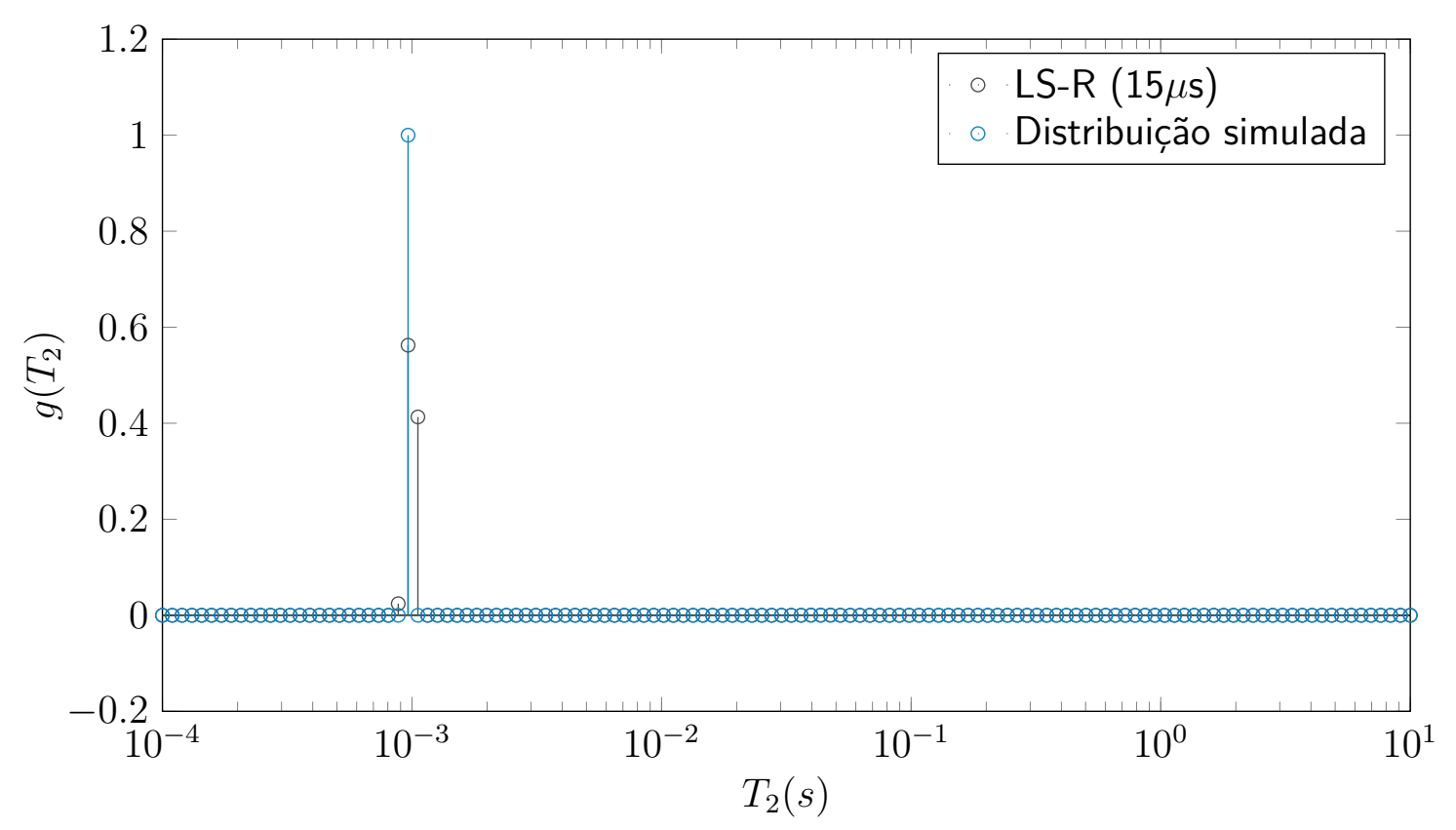

Fonte: Elaborada pelo autor.

Tabela 3.11 - Parâmetros utilizados e resultados obtidos para cada algoritmo no teste três.

\begin{tabular}{ccccccc} 
algoritmo & $\eta$ ou $h$ & $\lambda$ & iteraç̃oes & tempo(s) & MSE & $\sum\left(g\left(T_{2}\right)<0\right)^{2}$ \\
\hline \hline LMS-NN & 0,95 & - & 20000 & 2212 & $1,19.10^{-3}$ & 0 \\
\hline LMS-RNN & 0,95 & $10^{-5}$ & 614 & 26 & $5,74.10^{-3}$ & 0 \\
\hline Hopfield & $10^{-3}$ & - & 12529 & 130 & $6,55.10^{-3}$ & 0 \\
\hline LS-R (SVD) & - & $10^{-4}$ & 592 & 61 & $2,83.10^{-3}$ & $10^{-5}$ \\
\hline
\end{tabular}

Fonte: Elaborada pelo autor.

Das figuras 3.54, 3.55, 3.56 e 3.57 nota-se que todos os algoritmos identificaram a posição do pulso unitário, contudo apenas os resultados obtidos com o LMS-NN e o LS-R se aproximaram da distribuição original. Observando a tabela 3.11 nota-se o erro médio quadrático em relação a distribuição simulada permaneceu regular para todos os algoritmos. Entre os dois melhores resultados, LMS-NN e LS-R, o algoritmo dos mínimos quadrados regularizado apresentou convergência mais rápida. 


\subsection{Teste 4 - sinal real}

O quarto teste foi realizado com sinal de magnetização real obtido junto ao grupo de ressonância magnética do IFSC.

\subsubsection{LMS-NN}

Figura 3.58 - Distribuição de valores obtidas através do algoritmo LMS-RNN para sinal experimental real com $\tau^{*}=240 \mu s$.

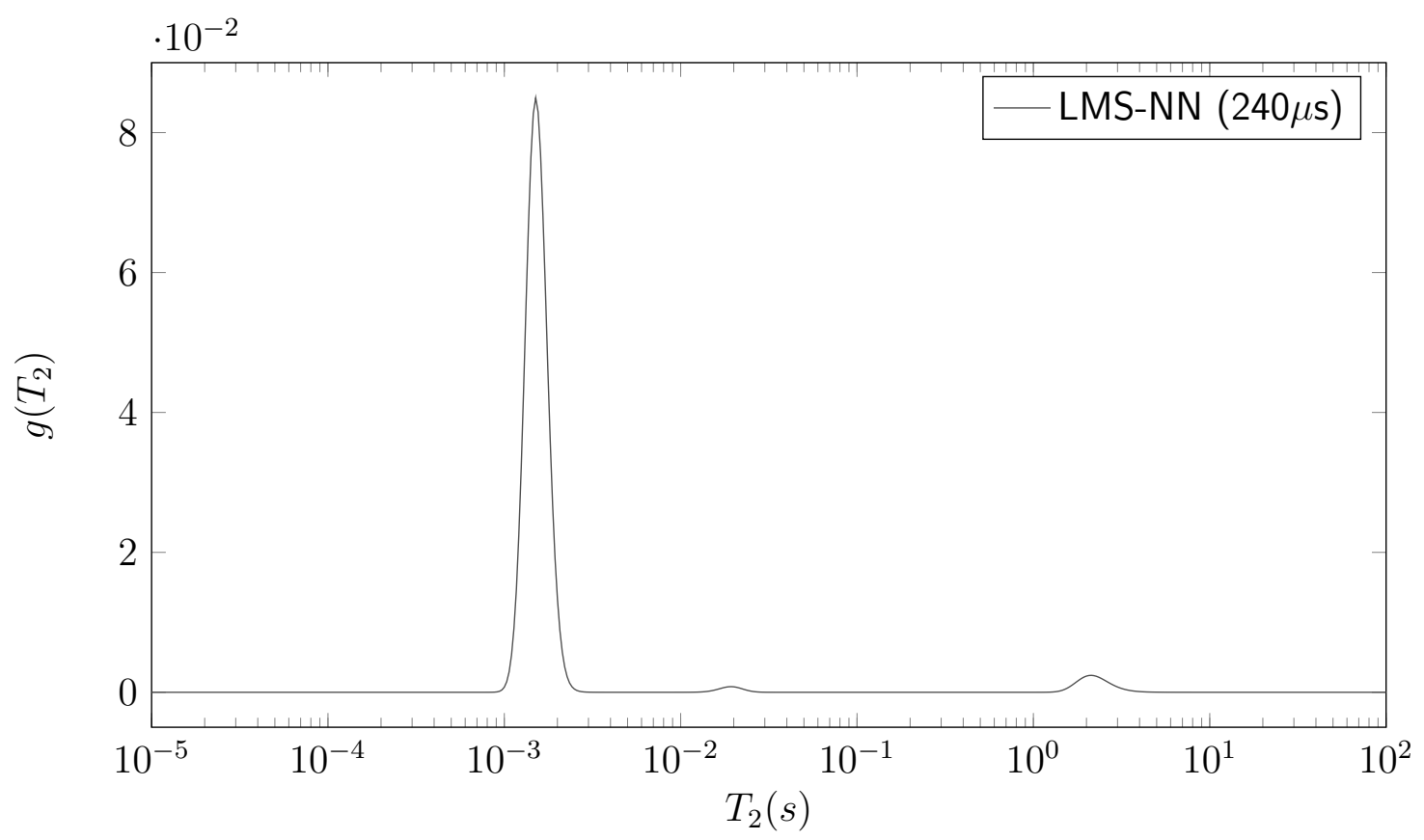

Fonte: Elaborada pelo autor.

Tabela 3.12 - Parâmetros utilizados e resultados obtidos com algoritmo de LMS-NN para o teste com sinal experimental real.

\begin{tabular}{ccccc}
$\tau^{*}$ & $\eta$ & iterações & tempo(s) & convergência média \\
\hline \hline $240 \mu s$ & $10^{-7}$ & 4043 & 301 & 1 \\
\hline $480 \mu s$ & $10^{-7}$ & 3750 & 150 & 1 \\
\hline $960 \mu s$ & $10^{-7}$ & 12540 & 396 & 1 \\
\hline $240 \mu s, 480 \mu s, 960 \mu s$ & $10^{-7}$ & 6071 & 172 & 1 \\
\hline
\end{tabular}

Fonte: Elaborada pelo autor. 
Figura 3.59 - Distribuição de valores obtidas através do algoritmo LMS-RNN para sinal experimental real com $\tau^{*}=480 \mu s$.

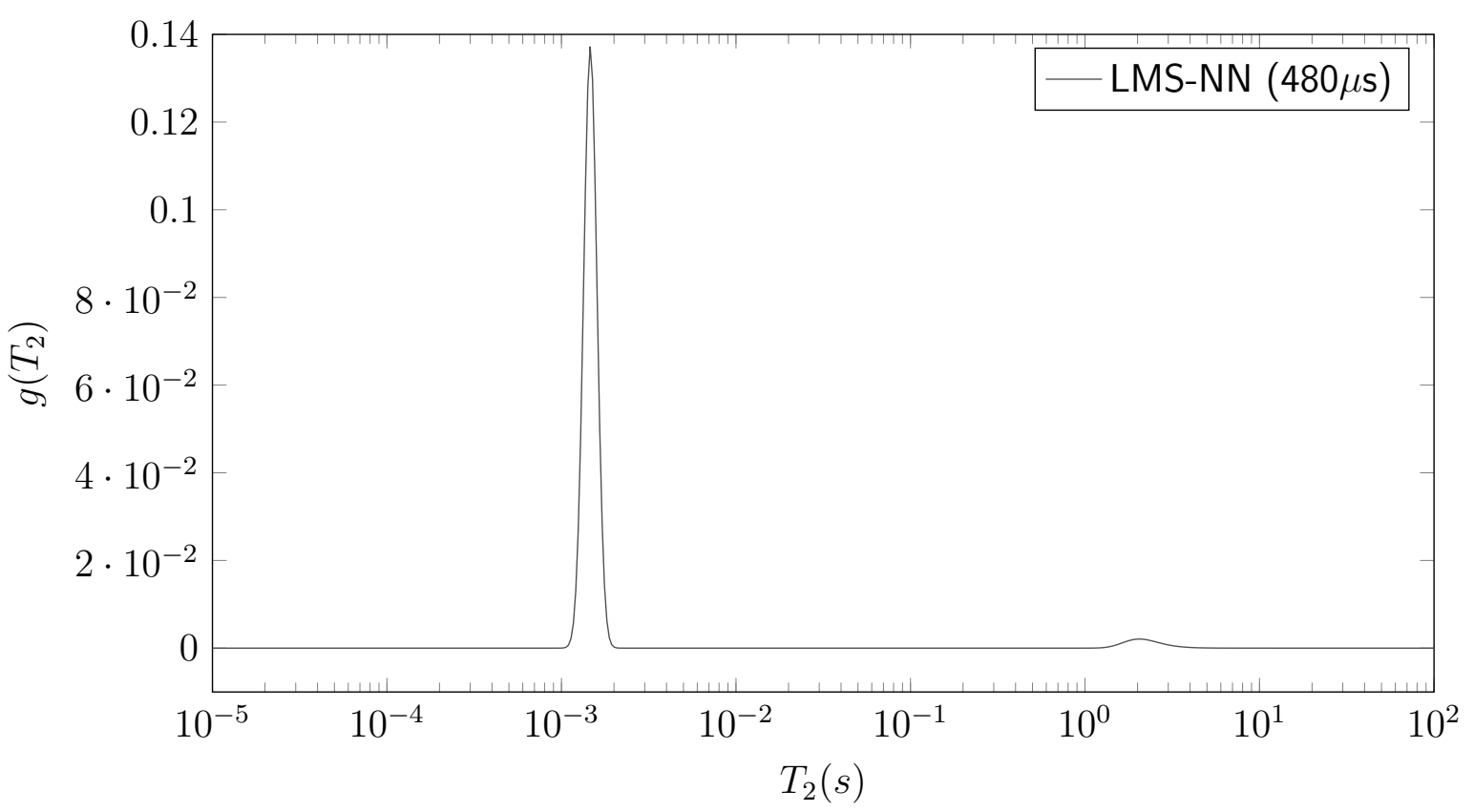

Fonte: Elaborada pelo autor.

Figura 3.60 - Distribuição de valores obtidas através do algoritmo LMS-NN para sinal experimental real com $\tau^{*}=960 \mu s$.

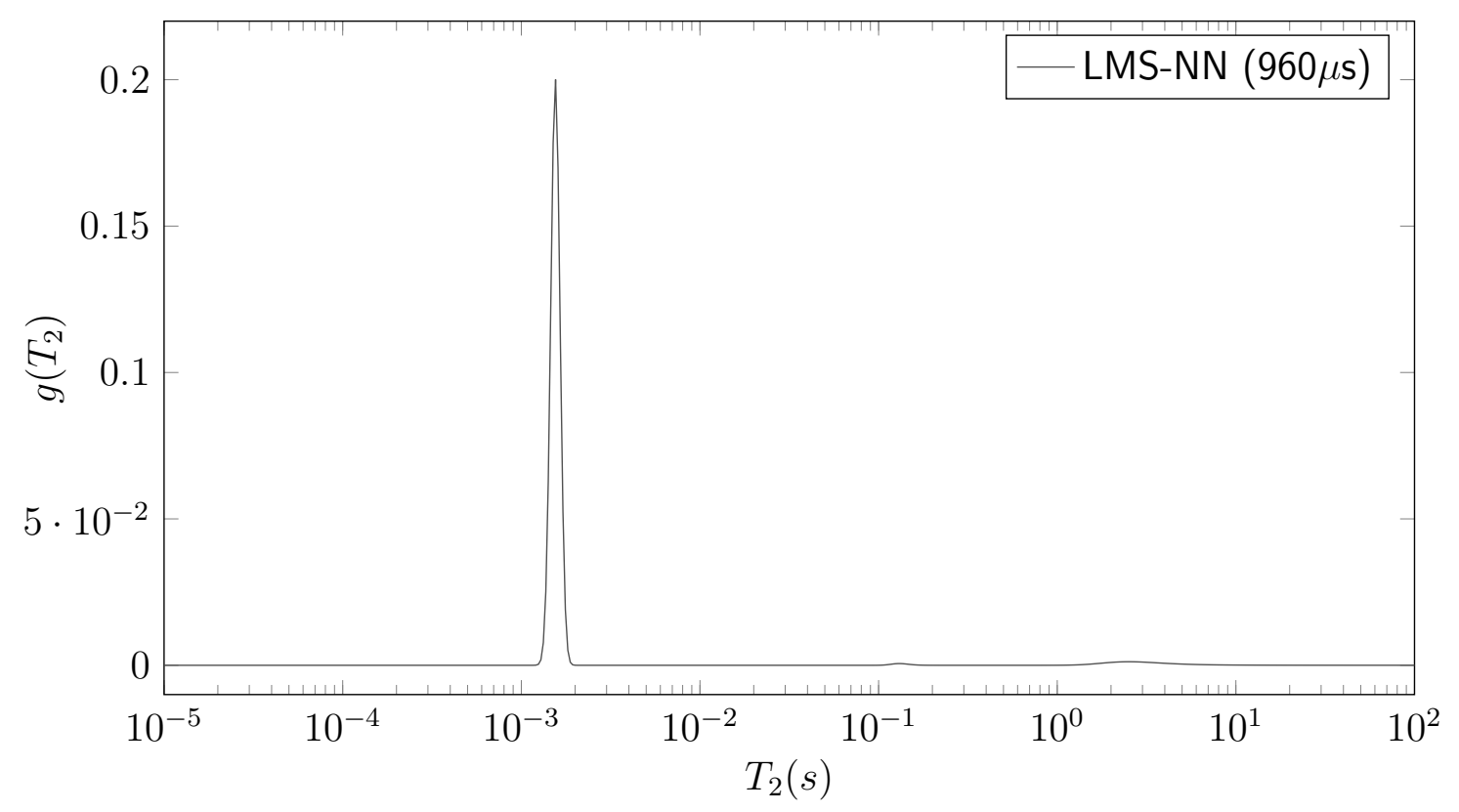

Fonte: Elaborada pelo autor. 
Figura 3.61 - Distribuição de valores obtida através do algoritmo LMS-NN combinando os sinais experimentais reais com $\tau^{*}=240 \mu s, 480 \mu \mathrm{s}$ e $960 \mu \mathrm{s}$.

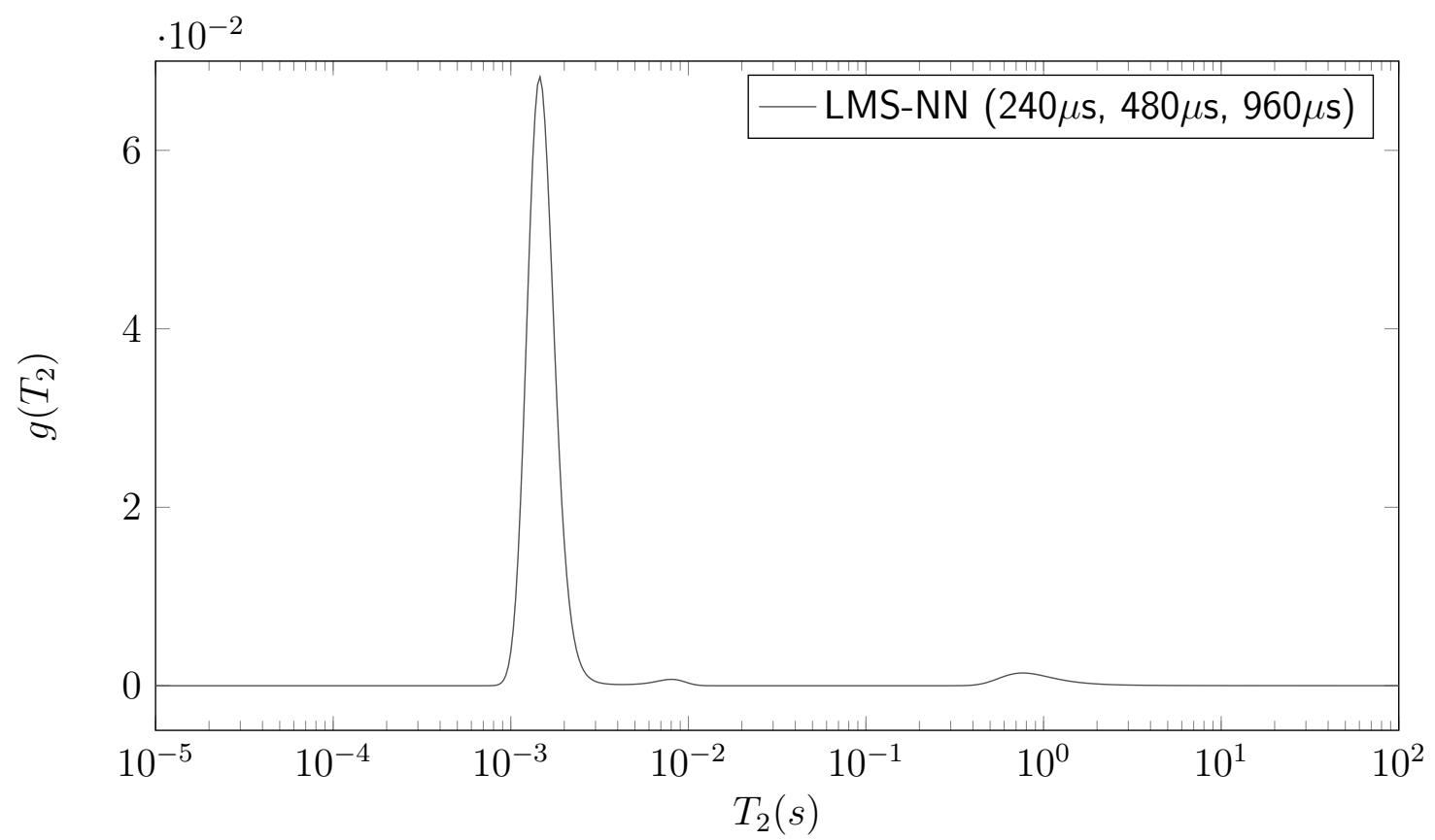

Fonte: Elaborada pelo autor. 


\subsubsection{LMS-RNN}

Figura 3.62 - Distribuição de valores obtidas através do algoritmo LMS-RNN para sinal experimental real $\operatorname{com} \tau^{*}=240 \mu \mathrm{s}$.

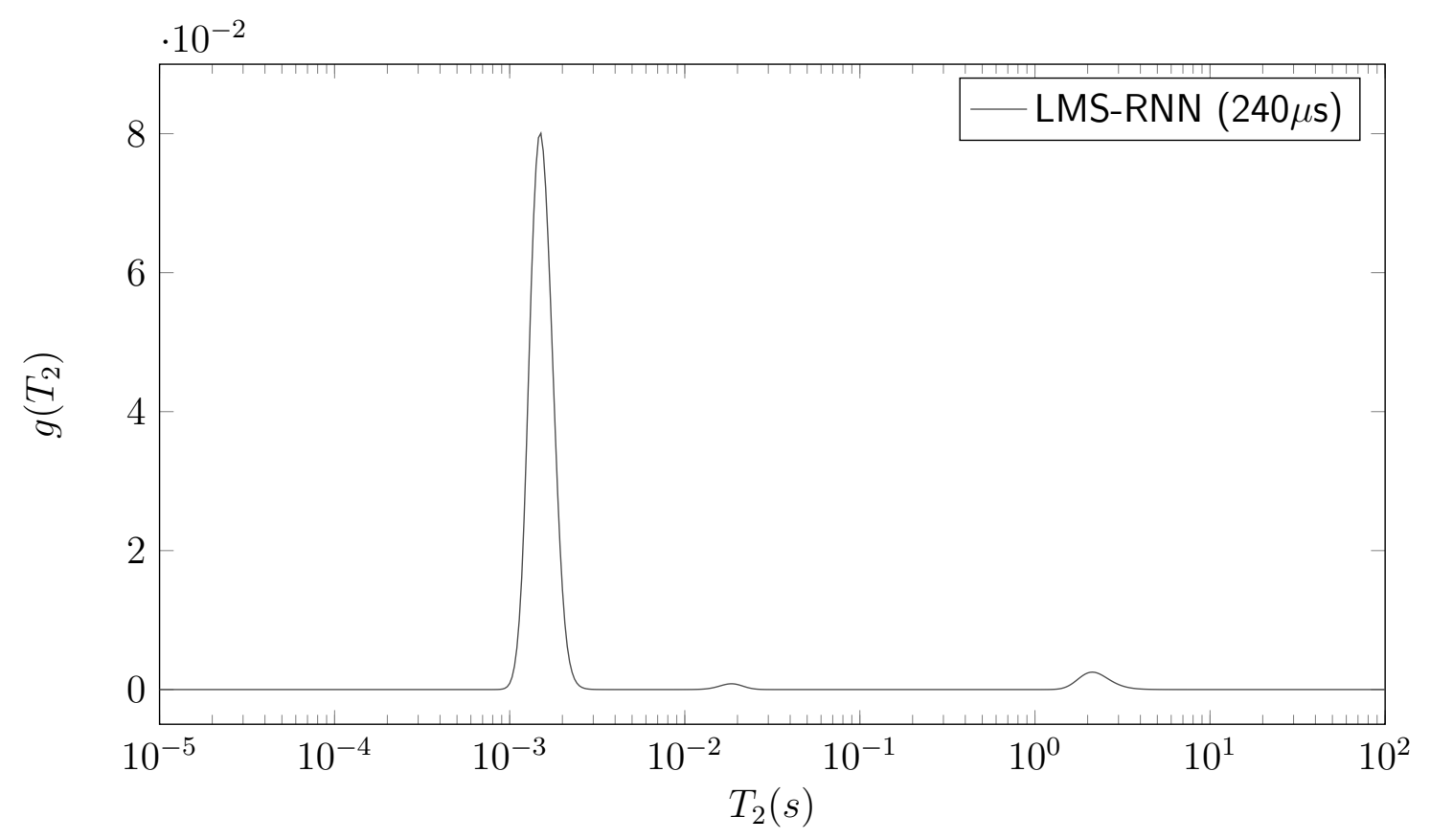

Fonte: Elaborada pelo autor.

Tabela 3.13 - Parâmetros utilizados e resultados obtidos com algoritmos LMS-RNN para o teste com sinal experimental real.

\begin{tabular}{cccccc}
$\tau^{*}$ & $\eta$ & $\lambda$ & iterações & tempo(s) & convergência média \\
\hline \hline $240 \mu s$ & $10^{-7}$ & 1 & 4350 & 440 & 1 \\
\hline $480 \mu s$ & $10^{-7}$ & 1 & 3770 & 214 & 1 \\
\hline $960 \mu s$ & $10^{-7}$ & 1 & 11825 & 413 & 1 \\
\hline $240 \mu s, 480 \mu s, 960 \mu s$ & $10^{-7}$ & 1 & 6071 & 210 & 1 \\
\hline
\end{tabular}

Fonte: Elaborada pelo autor. 
Figura 3.63 - Distribuição de valores obtidas através do algoritmo LMS-RNN para sinal experimental real com $\tau^{*}=480 \mu \mathrm{s}$.

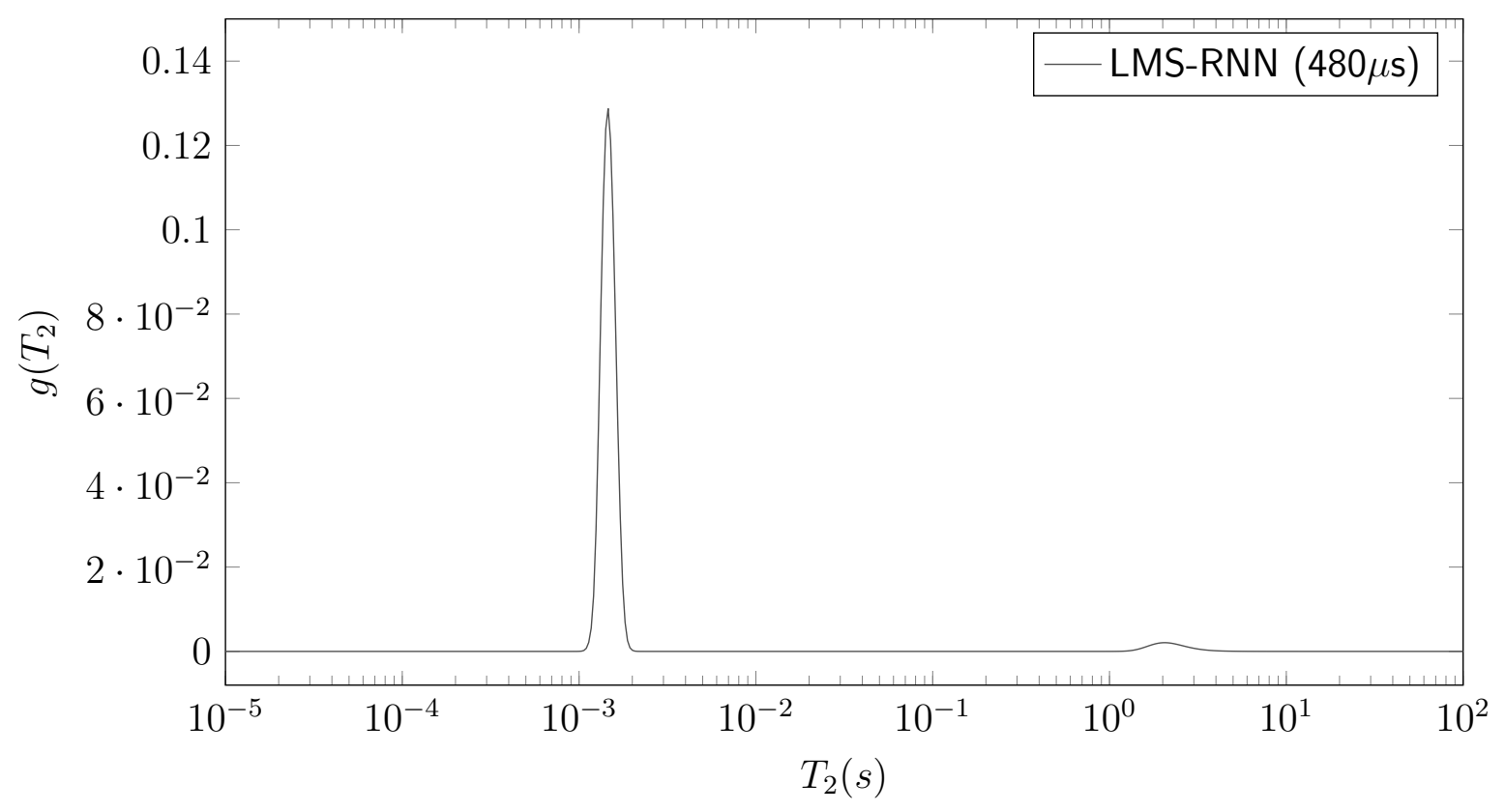

Fonte: Elaborada pelo autor.

Figura 3.64 - Distribuição de valores obtidas através do algoritmo LMS-RNN para sinal experimental real com $\tau^{*}=960 \mu \mathrm{s}$.

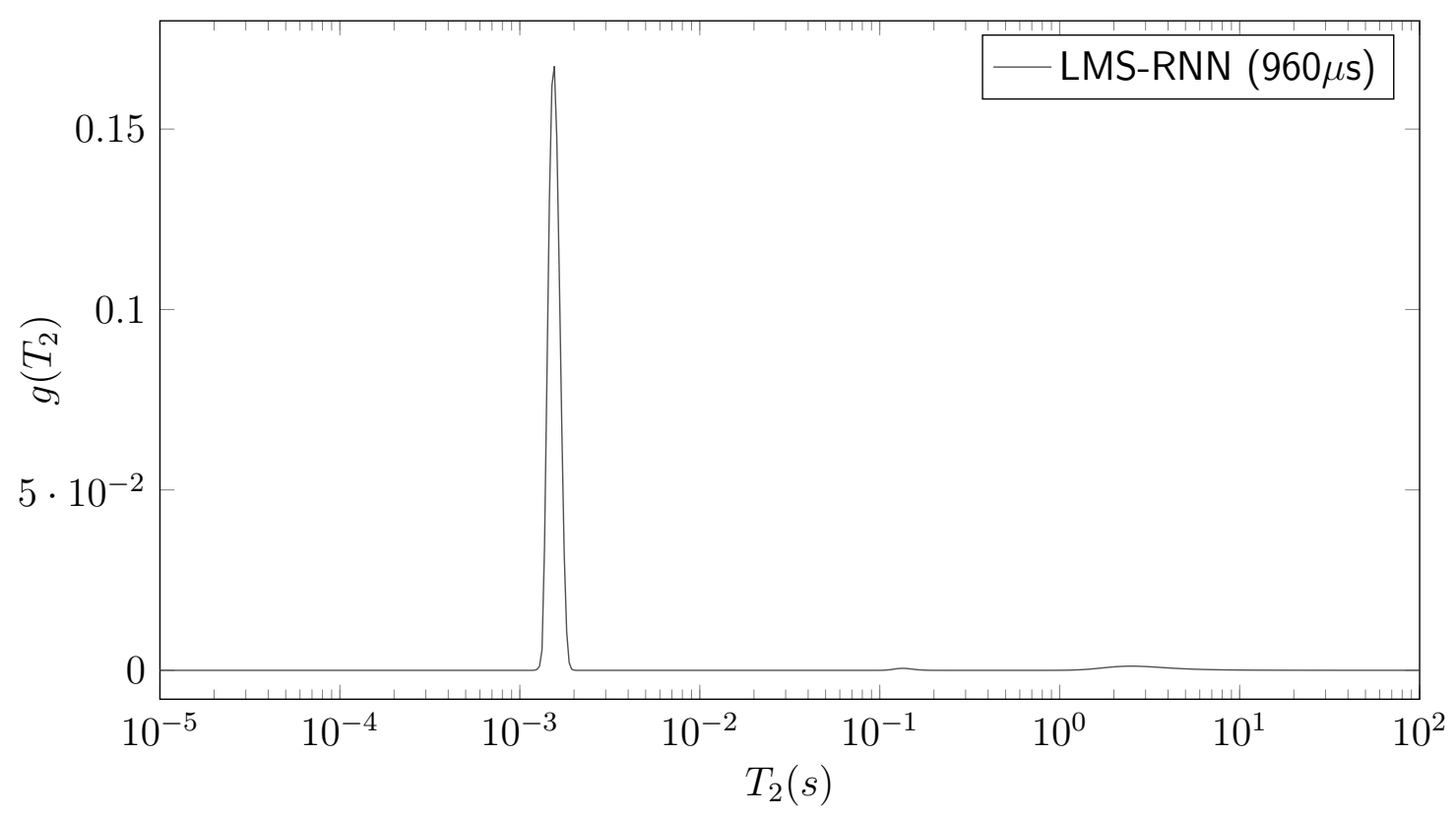

Fonte: Elaborada pelo autor. 
Figura 3.65 - Distribuição de valores obtida através do algoritmo LMS-RNN combinando os sinais experimentais reais com $\tau^{*}=240 \mu s, 480 \mu s$ e $960 \mu s$.

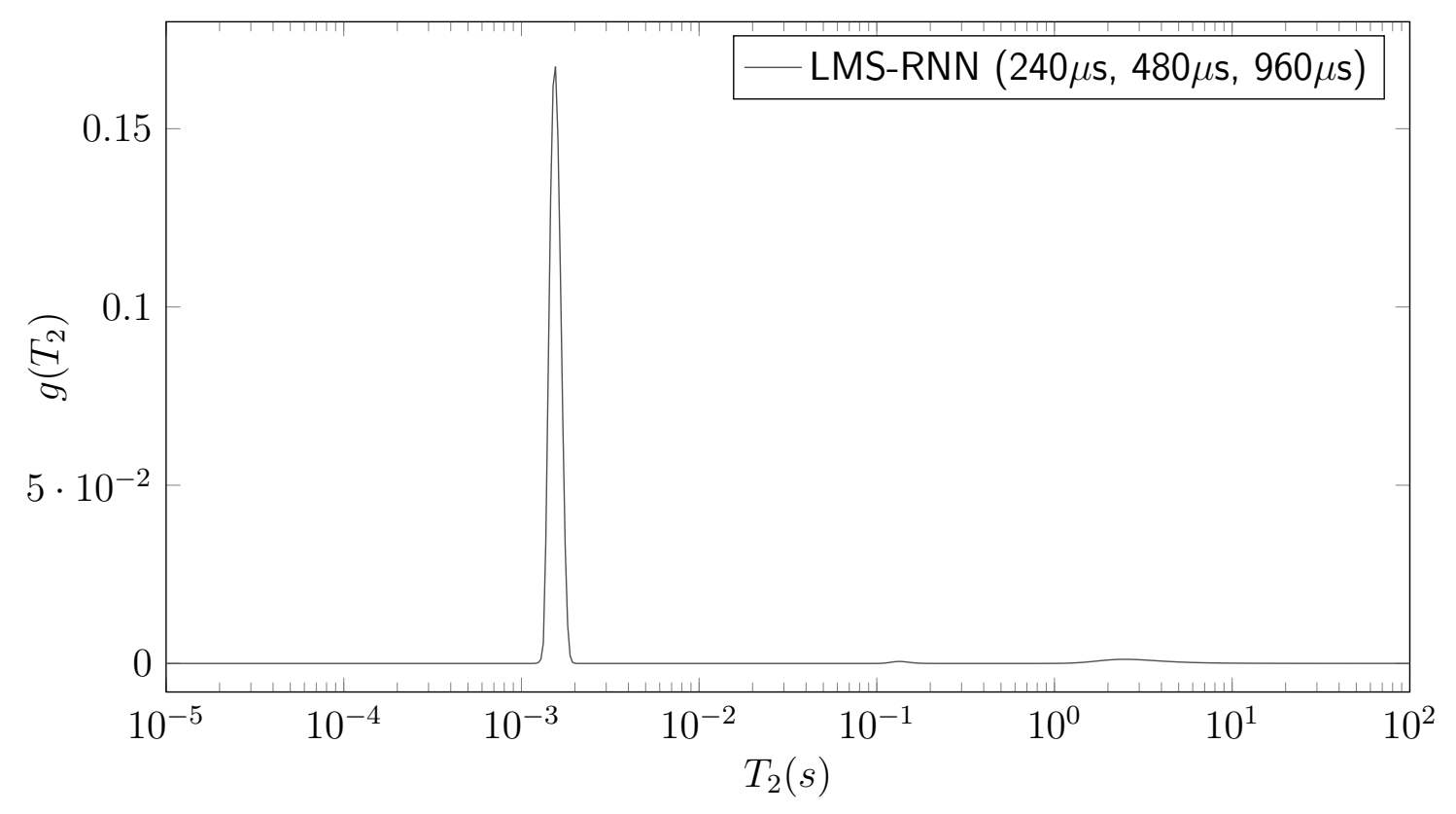

Fonte: Elaborada pelo autor. 


\subsubsection{Hopfield}

Figura 3.66 - Distribuição de valores obtidas através do algoritmo da rede recorrente de Hopfield para sinal experimental real com $\tau^{*}=240 \mu s$.

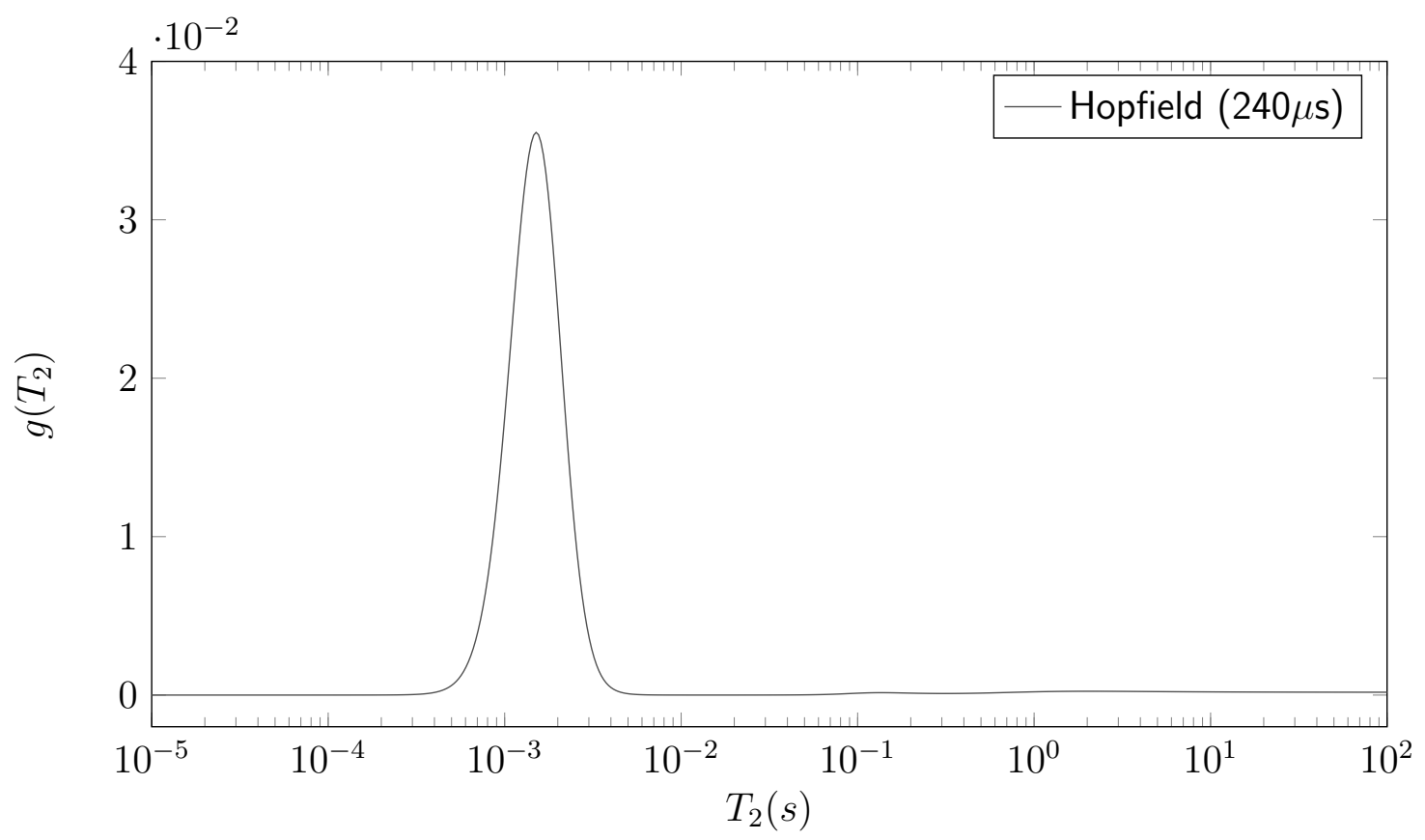

Fonte: Elaborada pelo autor.

Tabela 3.14 - Parâmetros utilizados e resultados obtidos com algoritmo da rede recorrente Hopfield para o teste com sinal experimental real.

\begin{tabular}{ccccc}
$\tau^{*}$ & $h$ & iterações & tempo(s) & convergência média \\
\hline \hline $240 \mu s$ & $1,5.10^{-3}$ & 34494 & 361 & $10^{-4}$ \\
\hline $480 \mu s$ & $1,5.10^{-3}$ & 1407 & 15 & $10^{-4}$ \\
\hline $960 \mu s$ & $1,5.10^{-3}$ & 1049 & 11 & $10^{-4}$ \\
\hline
\end{tabular}

Fonte: Elaborada pelo autor. 
Figura 3.67 - Distribuição de valores obtidas através do algoritmo da rede recorrente de Hopfield para sinal experimental real com $\tau^{*}=480 \mu s$.

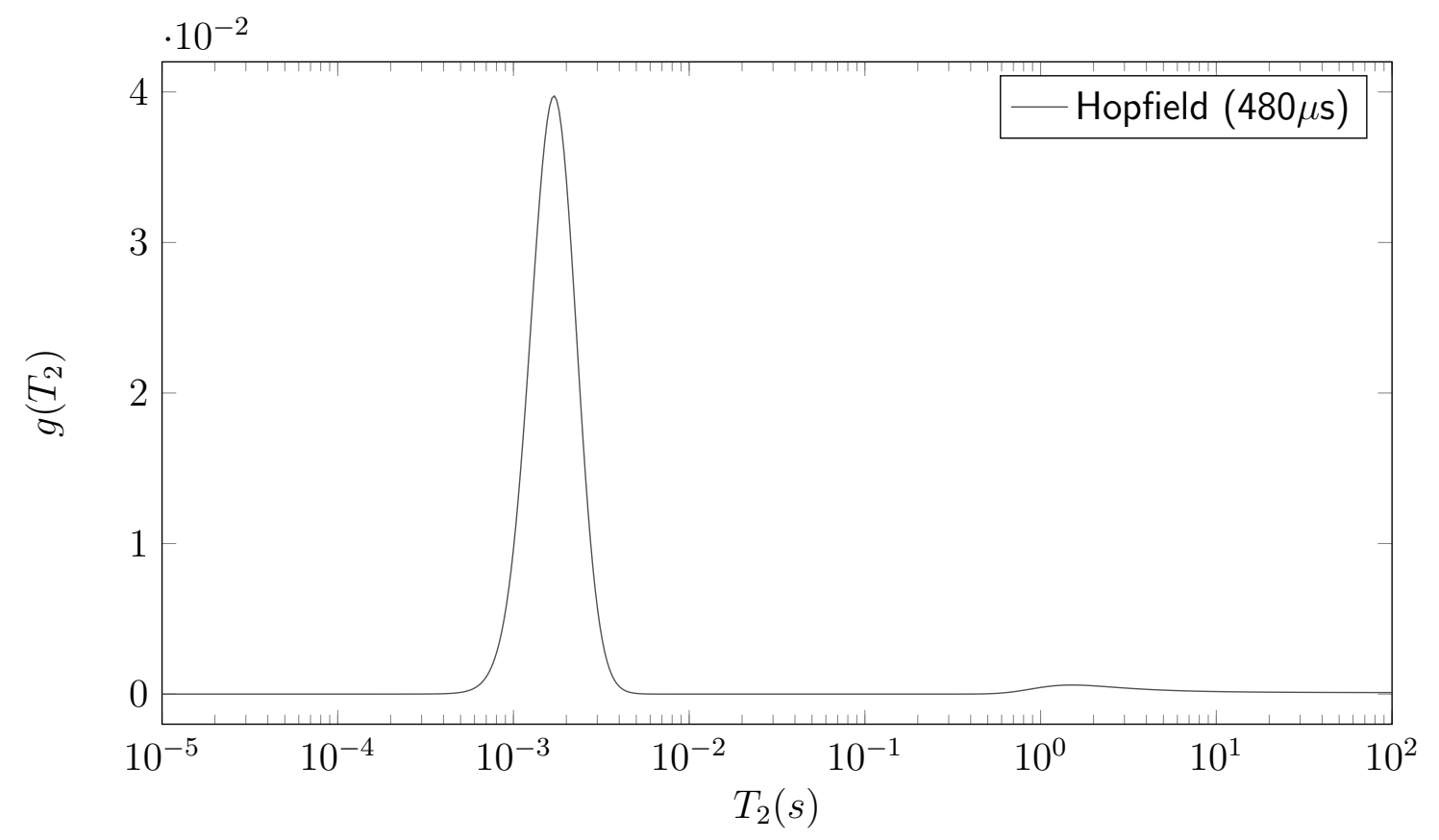

Fonte: Elaborada pelo autor.

Figura 3.68 - Distribuição de valores obtidas através do algoritmo da rede recorrente de Hopfield para sinal experimental real com $\tau^{*}=960 \mu s$.

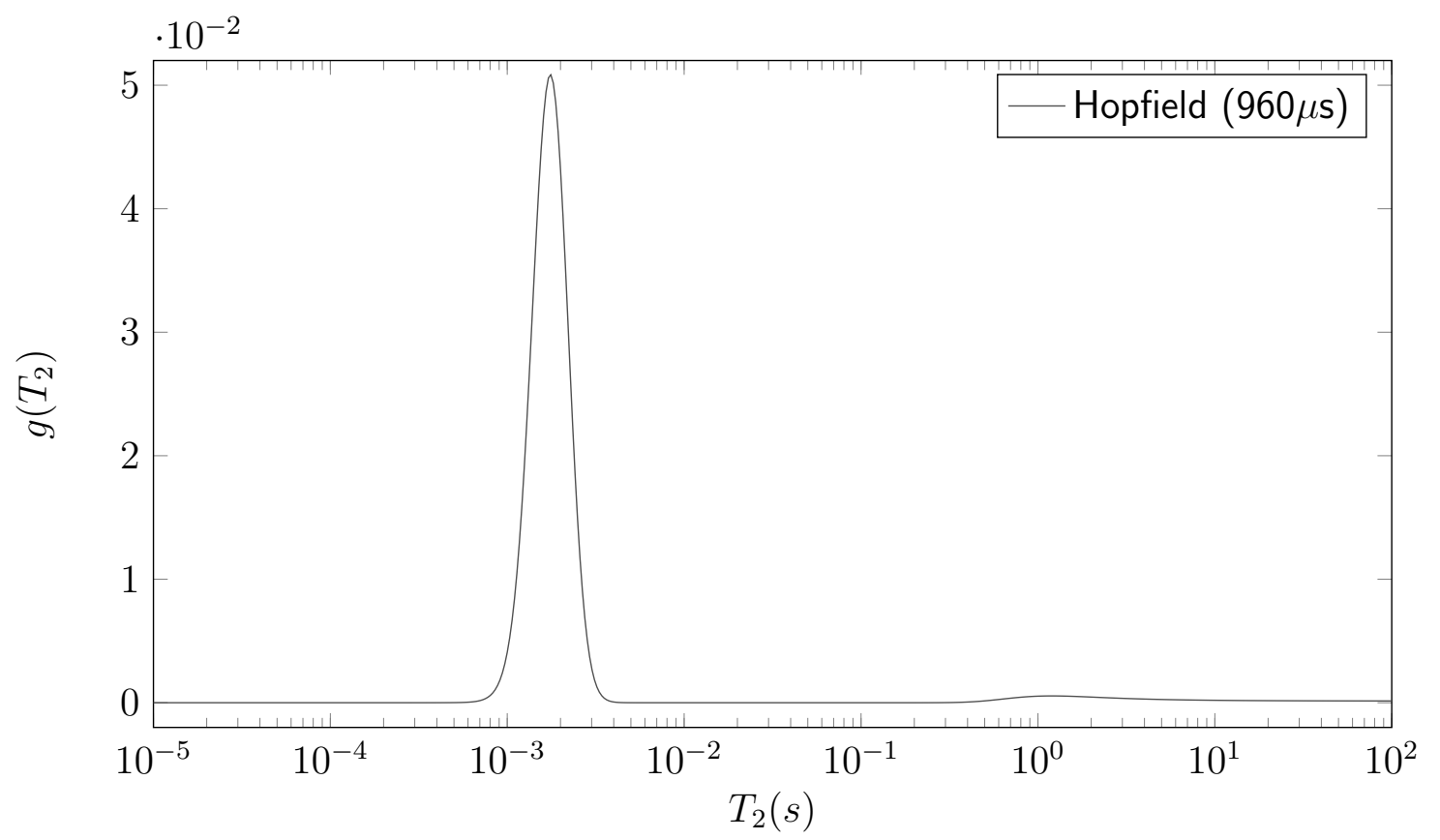

Fonte: Elaborada pelo autor. 


\subsubsection{LS-R (SVD)}

Figura 3.69 - Distribuição de valores obtidas através do algoritmo mínimos quadrados regularizado para sinal experimental real com $\tau^{*}=240 \mu s$.

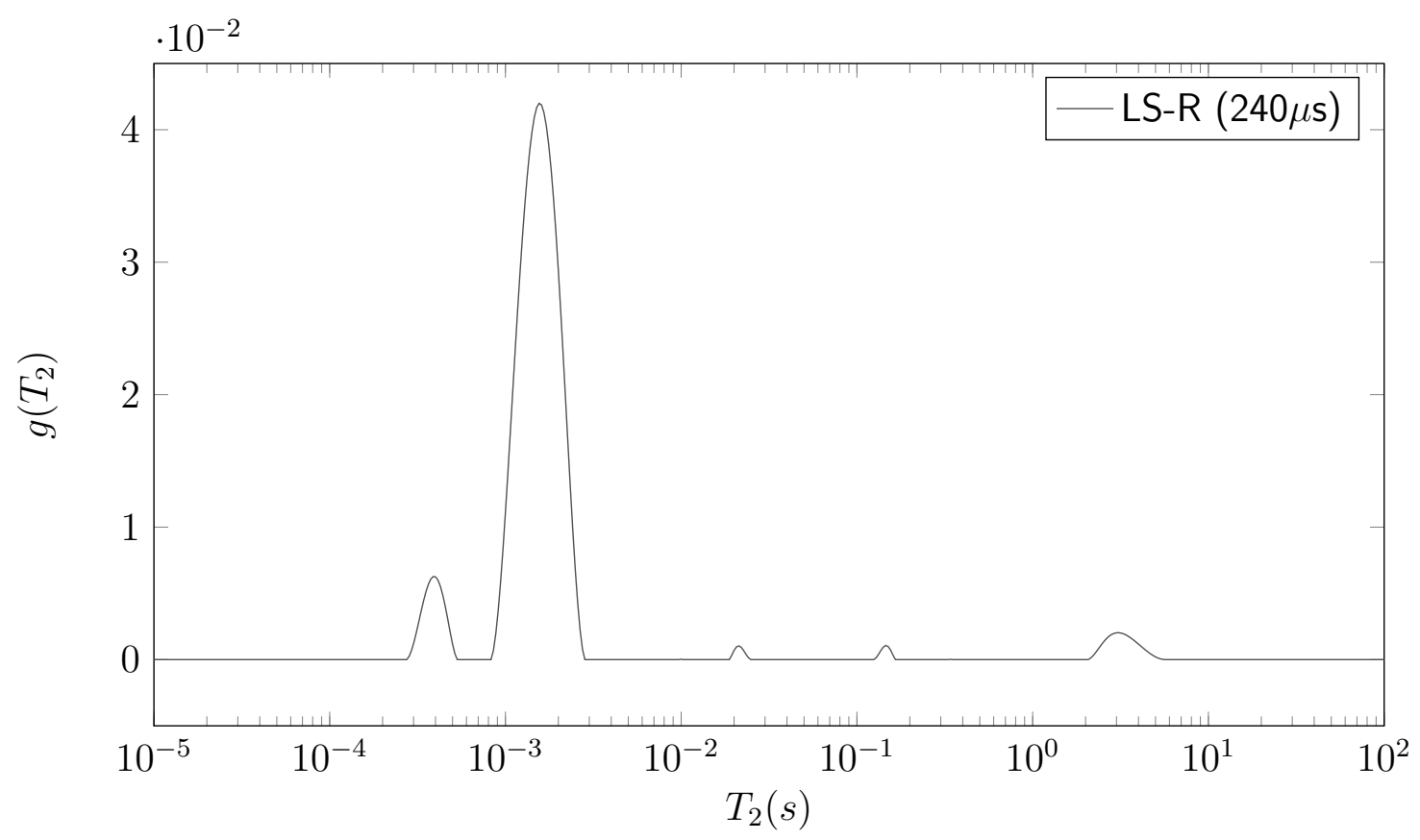

Fonte: Elaborada pelo autor.

Tabela 3.15 - Parâmetros utilizados e resultados obtidos com algoritmo dos mínimos quadrados regularizado para o teste com sinal experimental real.

\begin{tabular}{ccccc}
$\tau^{*}$ & $\lambda$ & iterações & tempo(s) & $\sum\left(g\left(T_{2}\right)<0\right)^{2}$ \\
\hline \hline $240 \mu s$ & 10 & 10 & 37 & $8,92.10^{-4}$ \\
\hline $480 \mu s$ & 10 & 76 & 314 & $9,81.10^{-4}$ \\
\hline $960 \mu s$ & 10 & 23 & 100 & $9,20.10^{-4}$ \\
\hline
\end{tabular}

Fonte: Elaborada pelo autor.

Dos resultados no teste utilizando sinal experimental real nota-se que, exceto pelas amplitudes e obviamente também as larguras, tendo em vista a normalização das distribuições, há bastante similaridade nos resultados obtidos por todos os algoritmos. A localização dos picos mais relevantes se manteve regular em todos as distribuições alcançadas. Os algoritmos LMS-NN e LMS-RNN apresentaram resultados bastante similares com pico relevante apresentando largura reduzida se comparado aos demais resultados e picos secundários razoavelmente definidos. A rede recorrente de Hopfield apresentou suavização mais acentuada que os demais 
Figura 3.70 - Distribuição de valores obtidas através do algoritmo mínimos quadrados regularizado para sinal experimental real $\operatorname{com} \tau^{*}=480 \mu s$.

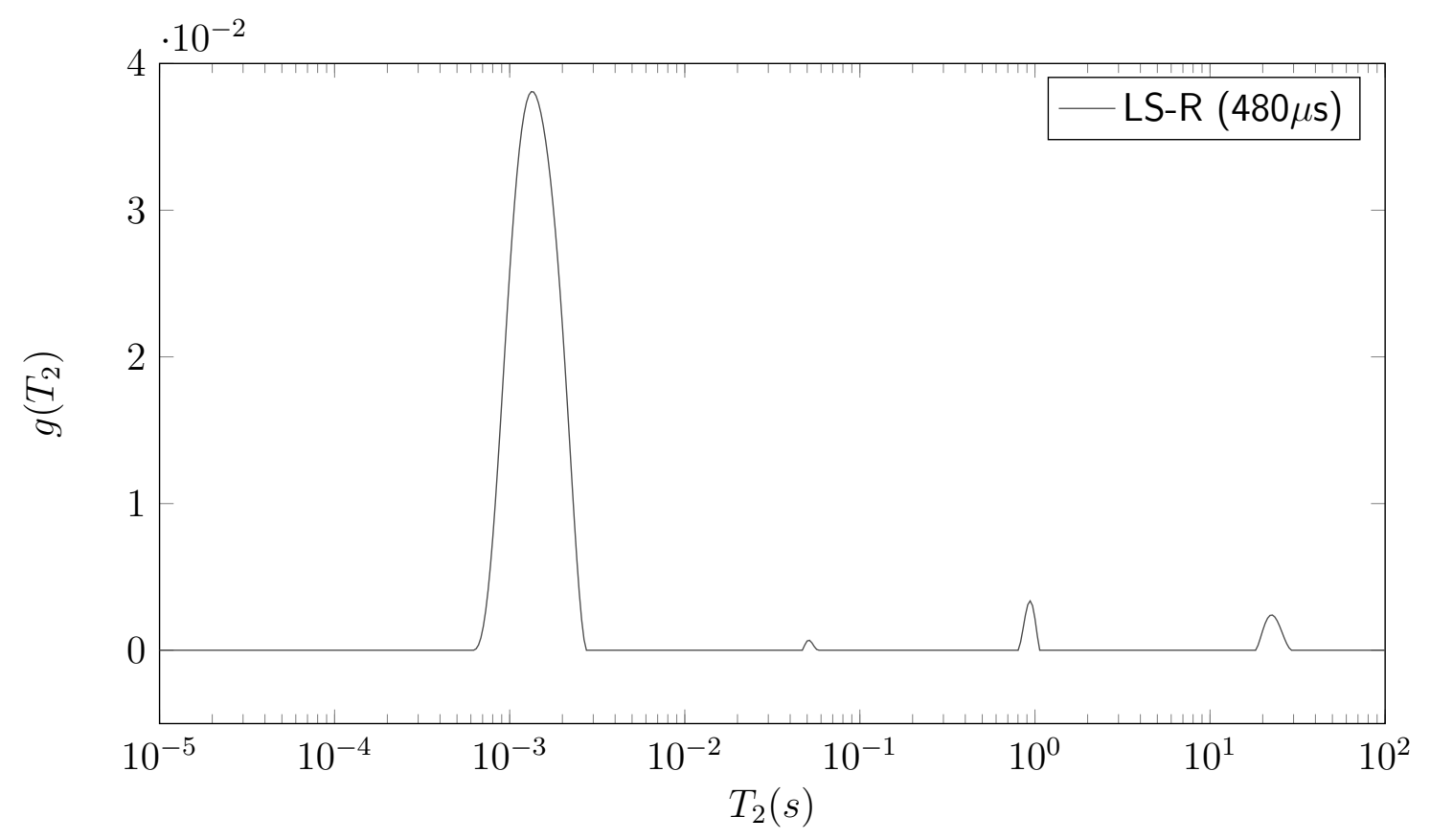

Fonte: Elaborada pelo autor.

Figura 3.71 - Distribuição de valores obtidas através do algoritmo mínimos quadrados regularizado para sinal experimental real com $\tau^{*}=960 \mu s$.

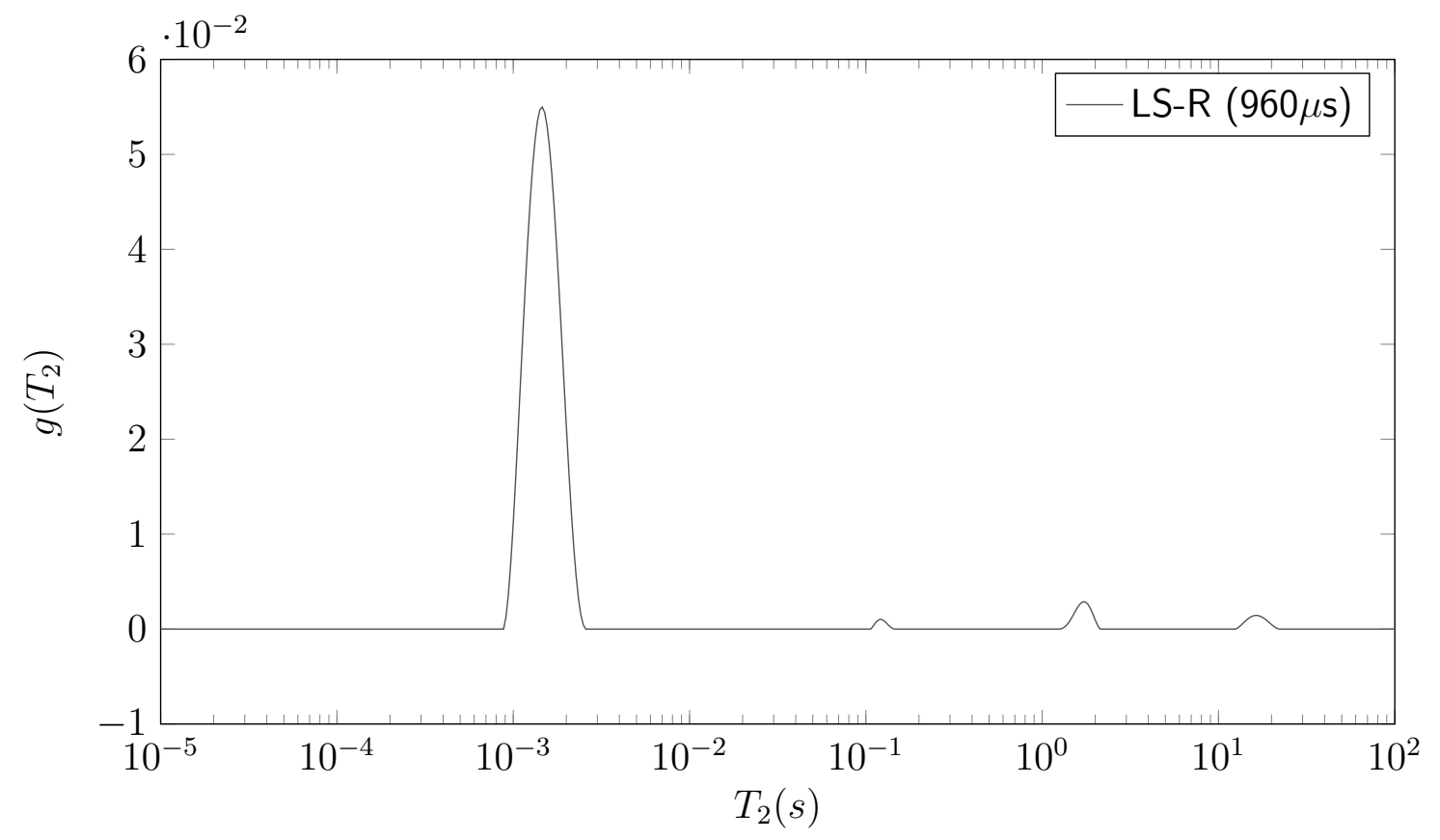

Fonte: Elaborada pelo autor.

conforme se pode observar nas figuras 3.66, 3.67 e 3.68. Já o algoritmo dos mínimos quadra- 
dos regularizado apresentou os resultados com pico principal e secundários mais bem definidos com todos os três sinais analisados. Contudo na figura 3.69 nota-se o aparecimento de um pico por volta de $8.10^{-4}$ segundos que não ocorreu em nenhum outro resultado. Consideranto o tempo médio de convergência a rede recorrente de Hopfield foi o algoritmo que obteve o melhor resultado com 129 segundos, seguido do LS-R com 150 segundos, LMS-NN com 254 segundos e finalmente o LMS-RNN com 319 segundos. Contudo deve-se atentar para o fato de que o baixo tempo de convergência do LS-R é devido a energia máxima relativamente elevada das componentes negativas do sinal de saída. 


\section{Conclusões e sugestões para trabalhos}

\section{futuros}

Neste capítulo seguem as conclusões obtidas a partir dos resultados demonstrados no capítulo anterior, destacando a aplicabilidade dos algoritmos. Além disso, sugestões para trabalhos futuros também são comentadas.

Pode-se dizer que a principal contribuição deste trabalho está relacionada com extensão da aplicabilidade dos métodos estudados e do alcance dos resultados obtidos. Devido a generalidade das equações integrais de Fredholm do primeiro tipo, os algoritmos estudados aqui são aplicáveis a qualquer situação onde determinado sinal possa ser definido na forma geral de uma equação integral de Fredholm do primeiro tipo e onde o núcleo desta equação seja previamente conhecido e medidas do sinal possam ser obtidas experimentalmente. Além disso, foi possível demonstrar também como métodos já bastante explorados em outras áreas podem ser combinados para se tornarem aplicáveis na solução de novos problemas. Os três métodos analisados, LMS-NN, LMS-RNN e a rede recorrente de Hopfield mostraram resultados bastante satisfatórios se comparados ao bem conhecido LS-R.

O algoritmo dos mínimos quadrados médios com restrição de não negatividade, de maneira geral, apresentou bons resultados na identificação das distribuições, principalmente através da combinação de conjuntos de sinais obtidos com diferentes tempos de eco. Dentre os testes realizados mostrou-se o melhor resultado em relação ao erro médio quadrático nas situações de descontinuidade, além de apresentar resultados bem definidos no teste com sinal real. A vantagem do LMS-NN frente ao LS-R e o LMS-RNN está relacionada principalmente com o fato de não ser necessário definir parâmetro de regularização, no entanto, por vezes, principalmente quando a análise envolve um único sinal, a falta de regularização tende a aumentar a complexidade das solução ou mesmo tornar a convergência substancialmente mais lenta.

O algoritmo dos mínimos quadrados médios com restrição de não negatividade baseado 
nos métodos de Karush-Kuhn-Tucker e regularização de complexidade de Tikhonov apresentou bons resultados em todos os testes, dentre os quais pode-se destacar o melhor resultando no teste com distribuição espaçada (considerando o erro médio quadrático em relação a distribuição simulada) e resultados muito bem definidos no teste com sinal real. Assim como no LMS-NN os melhores resultados estão relacionados à análise com conjuntos de sinais obtidos com diferentes tempos de eco. A principal desvantagem do método frente ao LS-R e o LMSNN está na necessidade de ajuste de dois parâmetros, o parâmetro da taxa de convergência $\eta$ e o parâmetro que controla a complexidade da solução $\lambda$, no entanto, observou-se durante os testes que a influência do $\lambda$ no LMS-RNN é muito pequena se comparado ao LS-R, por vezes até não há diferenças visíveis na distribuição alcançada, contudo mostrou-se positiva no que diz respeito a convergência do algoritmo, mesmo com valores pequenos.

A rede recorrente de Hopfield mostrou excelentes resultados no primeiro teste, isto é, na identificação de distribuições suaves e sem espaçamentos, contudo mostrou-se ineficaz frente a situações mais complexas como nas distribuições espaçadas e nas situações de descontinuidade. As principais vantagens observadas na rede recorrente de Hopfield em relação aos outros algoritmos são o alto grau de paralelismo que pode ser alcançado na implementação e a existência de um único parâmetro $h$ relativamente fácil de definir.

O algoritmo dos mínimos quadrados com regularização de Tikhonov e decomposição em valores singulares, conforme já era esperado, apresentou bons resultados em todos os testes, é fato, entretanto, que a determinação do parâmetro de regularização $\lambda$ pode se tornar uma tarefa complicada e por vezes comprometer o resultado final da análise, além da impossibilidade de restringir totalmente o aparecimento de valores negativos na distribuição.

Do exposto acima a conclusão a qual se chega analisando todo o cenário é que os quatro algoritmos analisados são de fato aplicáveis a todas as situações, contudo em certos casos alguns apresentam comportamento melhor do que outros e infelizmente prever estes casos até o momento não é uma possibilidade, pode-se dizer portanto, que os métodos são complementares e permitem uma visão bem mais ampla e completa do problema e da solução se utilizados em conjunto.

Além dos métodos discutidos e demonstrados neste trabalho houve ainda tentativas de obter distribuições através de redes neurais artificiais utilizando abordagem de aprendizagem supervisionada, isto é, aprendizado por meio de pares de sinais rotulados e posterior inversão através unicamente da experiência adquirida pela rede neural. Foram testadas redes de múltiplas camadas (duas e três camadas ocultas) utilizando aprendizagem supervisionada através do algoritmo conhecido como retro propagação de erro e redes de função de base radial com 
aprendizagem supervisionada na determinação dos pesos sinápticos da camada de entrada da rede (utilizando também o algoritmo de retro propagação de erro) e não supervisionada na definição dos centros das função de base. Entretanto, durante o andamento da pesquisa notou-se diversas limitações da abordagem frente as características do problema em estudo, dentre as quais pode-se destacar a característica de filtragem associada ao núcleo da equações de Fredholm do primeiro tipo e as características do problema inverso mal-posto, deste modo a principal sugestão para trabalhos futuros é evitar este tipo de abordagem comum na análise dos sinais. 


\section{REFERÊNCIAS}

1 BLOCH, F. Nuclear induction. Physical Review, v. 70, n. 7/8, p. 460-474, 1946.

2 PURCELL, E. M.; TORREY, H. C.; POUND, R. V. Resonance absorption by nuclear magnetic moments in a solid. Physical Review, v. 69, n. 1/2, p. 37-38, 1946.

3 D'EURYDICE, M. N. Desenvolvimento de metodologias para o estudo de meios porosos por ressonância magnética nuclear. 2011. 172 p. Tese (Doutorado em Física) - Instituto de Física de São Carlos, Universidade de São Paulo, São Carlos, 2011.

4 KUPERMAN, V. Magnetic resonance imaging: physical principles and applications. San Diego: Academic Press, 2000.

5 GUIMARAES, A. Magnetismo e ressonância magnética em sólidos. São Paulo: EDUSP, 2009.

6 VOLD, R. L.; WAUGH, J. S.; KLEIN, M. P.; PHELPS, D. E. Measurement of spin relaxation in complex systems. Journal of Chemical Physics, New York, v. 48, n. 8, 1968. doi: 10.1063/1.1669699.

7 ZUCCHI, M. D. R. Implementação da técnica de espectroscopia in vivo por RMN e sua aplicação na fisiologia do exercício. 1997. 79 p. Dissertação (Mestrado em Ciências) - Instituto de Física de São Carlos, Universidade de São Paulo, São Carlos, 1997.

8 MCDONALD, G. G.; LEIGH JR., J. S. A new method for measuring longitudinal relaxation times. Journal of Magnetic Resonance (1969), v. 9, n. 3, p. 358 - 362, 1973.

9 HAHN, E. L. Spin echoes. Physical Review, v. 80, n. 4, p. 580-594, 1950.

10 CARR, H. Y.; PURCELL, E. M. Effects of diffusion on free precession in nuclear magnetic resonance experiments. Physical Review, v. 94, n. 3, p. 630-638, 1954.

11 MEIBOOM, S.; GILL, D. Modified spin-echo method for measuring nuclear relaxation times. Review of Scientific Instruments, v. 29, n. 8, p. 688-691, 1958.

12 COATES, G. R.; XIAO, L.; PRAMMER, M. G. Nmr logging: principles and applications. Houston: Gulf Professional Publishing, 1999.

13 DUNN, K.; BERGMAN, D.; LATORRACA, G. Nuclear magnetic resonance: petrophysical and logging applications. Amsterdam: Pergamon, 2002. 
14 BROWNSTEIN, K. R.; TARR, C. E. Importance of classical diffusion in nmr studies of water in biological cells. Physical Review A, v. 19, n. 6, p. 2446-2453, 1979.

15 VALFOUSKAYA, A.; ADLER, P. M.; THOVERT, J.-F.; FLEURY, M. Nuclear magnetic resonance diffusion with surface relaxation in porous media. Journal of Colloid and Interface Science, v. 295, n. 1, p. 188-201, 2006.

16 ARFKEN, G. B.; WEBER, H. J.; HARRIS, F. E. Mathematical methods for physicists. 6th ed. Boston: Academic Press, 2005.

17 HADAMARD, J. Sur les problèmes aux dérivés partielles et leur signification physique. Princeton University Bulletin, v. 13, p. 49-52, 1902.

18 HANSEN, P. C. Rank-deficient and discrete ill-posed problems: numerical aspects of linear inversion. Philadelphia: SIAM.

19 WIDROW, B.; STEARNS, S. D. Adaptive signal processing. Upper Saddle River, NJ, USA: Prentice-Hall, 1985.

20 FARHANG-BOROUJENY, B. Adaptive filters: theory and applications. New York: John Wiley \& Sons, 1998.

21 DA SILVA, I.; SPATTI, D.; FLAUZINO, R. Redes neurais artificiais para engenharia e ciências aplicadas: curso prático. São Paulo: Artliber, 2010.

22 HAYKIN, S. Adaptive filter theory. 3rd ed. Upper Saddle River, NJ, USA: Prentice-Hall, Inc., 1996.

23 DINIZ, P. Adaptive filtering: algorithms and practical implementation. New York: Springer, 1997.

24 WANG, Z. D.; XIAO, L. H.; LIU, T. Y. A new method for multi-exponential inversion of nmr relaxation measurements. Science in China Series $G$ - physics, mechanics \& astronomy, v. 47, n. 3 , p. $265-276,2004$.

25 CHEN, J.; RICHARD, C.; BERMUDEZ, J.; HONEINE, P. Nonnegative least-mean-square algorithm. IEEE Transactions on Signal Processing, v. 59, n. 11, p. 5225-5235, 2011.

26 BOYD, S.; VANDENBERGHE, L. Convex optimization. New York, NY: Cambridge University Press, 2004.

27 CHEN, J.; RICHARD, C.; BERMUDEZ, J.; HONEINE, P. A modified nonnegative Ims algorithm and its stochastic behavior analysis. c2011. 542-546 p. Disponível em:<http://ieeexplore.ieee.org/stamp/stamp.jsp?arnumber $=6190060>$.Acesso em: 10 mar. 2015. 
28 CHEN, J.; RICHARD, C.; HONEINE, P.; LANTÉRI, H.; THEYS, C. System identification under non-negativity constraints. In: EUROPEAN SIGNAL PROCESSING CONFERENCE, 18., 2010, Aalborg, Denmark. Proceedings ... Aalborg, Denmark: EURASIP, c2010. 1728$1732 \mathrm{p}$.

29 TIKHONOV, A. N. Solution of incorrectly formulated problems and the regularization method. Soviet Mathematics Doklady, v. 5, p. 1035-1038, 1963.

30 PHILLIPS, D. L. A technique for the numerical solution of certain integral equations of the first kind. Journal of the ACM, v. 9, n. 1, p. 84-97, 1962.

31 DREYFUS, G. Neural networks: methodology and applications. New York: Springer, 2005.

$32 \mathrm{HIRSCH}, \mathrm{M}$. W. Convergent activation dynamics in continuous time networks. Neural Networks, v. 2, n. 5, p. 331-349, 1989.

33 HOPFIELD, J. J. Neural networks and physical systems with emergent collective computational abilities. Proceedings of the National Academy of Sciences, v. 79, n. 8, p. 2554-2558, 1982.

34 HOPFIELD, J. J. Neurons with graded response have collective computational properties like those of two-state neurons. Proceedings of the National Academy of Sciences, v. 81, n. 10, p. 3088-3092, 1984.

35 AO, R. S.; BRAGA, J. Retrieval of transverse relaxation time distribution from spin-echo data by recurrent neural network. Journal of Magnetic Resonance, v. 177, n. 1, p. 146-151, 2005.

36 VEMURI, V.; JANG, G.-S. Inversion of fredholm integral equations of the first kind with fully connected neural networks. Journal of the Franklin Institute, v. 329, n. 2, p. 241-257, 1992.

37 TREFETHEN, L.; BAU, D. Numerical linear algebra. Philadelphia: Philadelphia Society for Industrial and Applied Mathematics, 1997.

38 GOLUB, G.; KAHAN, W. Calculating the singular values and pseudo-inverse of a matrix. Journal of the Society for Industrial \& Applied Mathematics Series B: numerical analysis, v. 2, n. 2, p. 205-224, 1965.

39 HANSEN, P. C.; O'LEARY, D. P. The use of the l-curve in the regularization of discrete ill-posed problems. SIAM Journal on Scientific Computing, v. 14, n. 6, p. 1487-1503, 1993.

40 HANSEN, P. C. The l-curve and its use in the numerical treatment of inverse problems. $1999 . \quad$ Disponível em: 
$<$ https://www.sintef.no/globalassets/project/evitameeting/2005/lcurve.pdf $>$.

Acesso em: 12 mar. 2015.

41 WILSON, J. Statistical approach to the solution of first-kind integral equations arising in the study of materials and their properties. Journal of Materials Science, v. 27, n. 14, p. 3911-3924, 1992.

42 PROVENCHER, S. W. A constrained regularization method for inverting data represented by linear algebraic or integral equations. Computer Physics Communications, v. 27, n. 3, p. 213-227, 1982.

43 PRESS, W. H.; TEUKOLSKY, S. A.; VETTERLING, W. T.; FLANNERY, B. P. Numerical recipes: the art of scientific computing. 3rd ed. New York, NY, USA: Cambridge University Press, 2007.

44 OPENMP ARCHITECTURE REVIEW BOARD. OpenMP application program interface version 4.0. 2013. Disponível em: <http://www.openmp.org/mpdocuments/OpenMP4.0.0.Examples.pdf>. Acesso em: 12 mar. 2015. 
APÊNDICE $A$

\section{Código $C++$ para $L M S-N N$ e LMS-RNN}

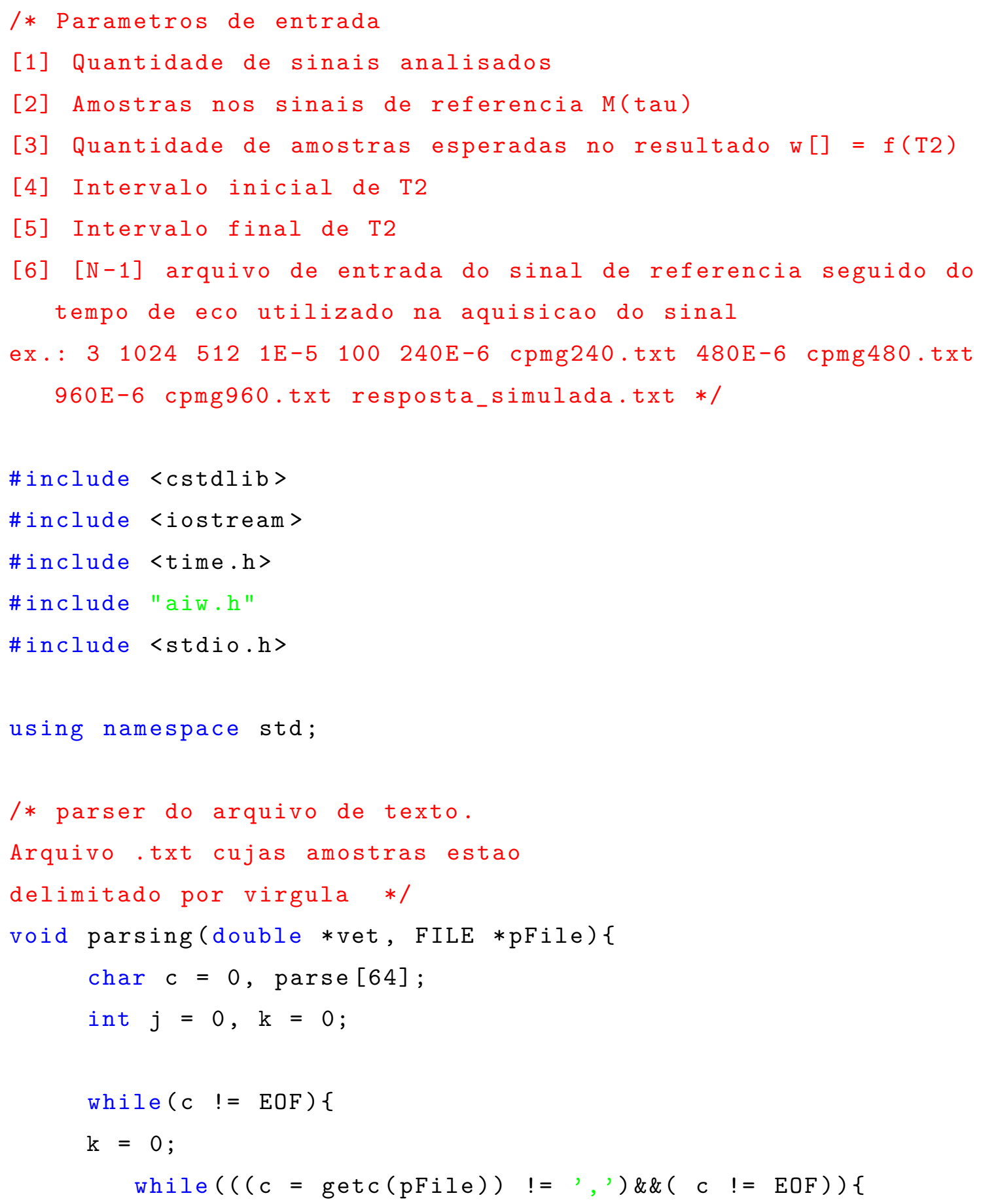




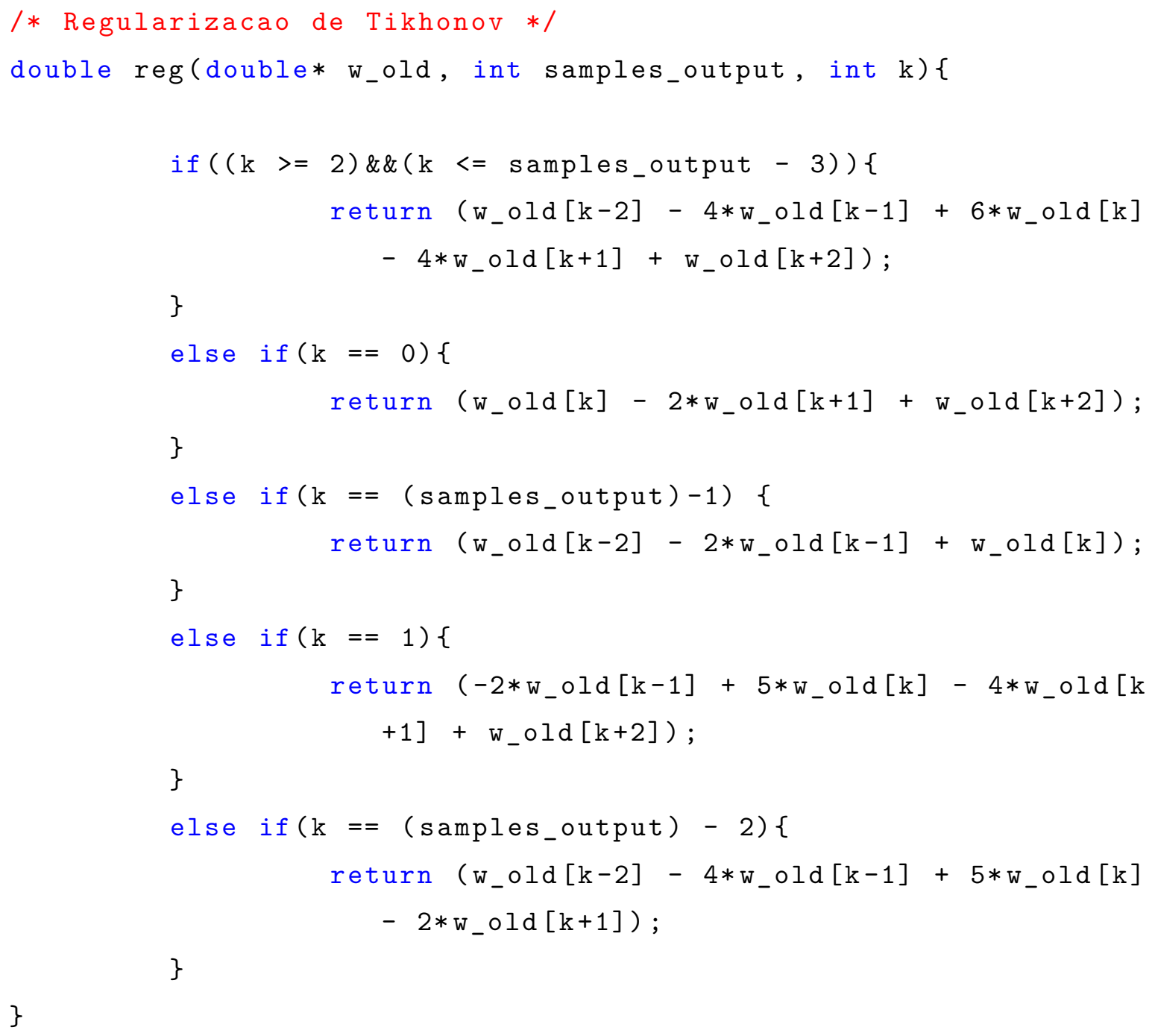




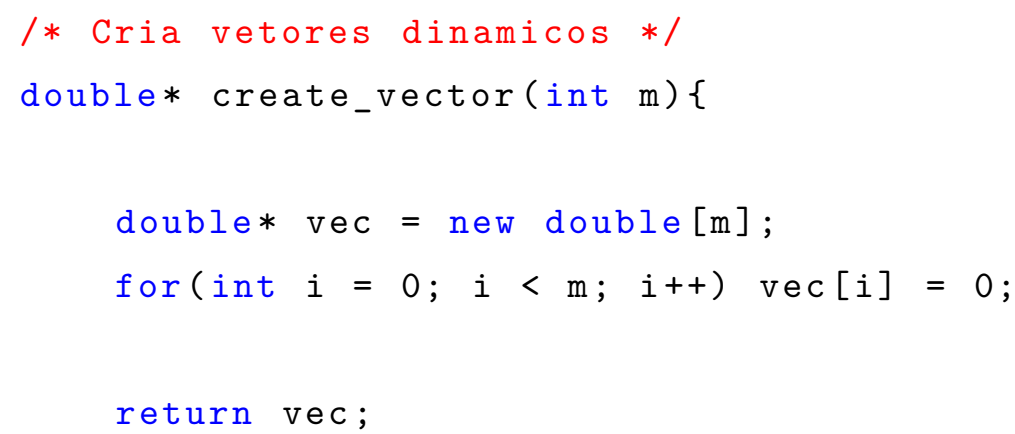




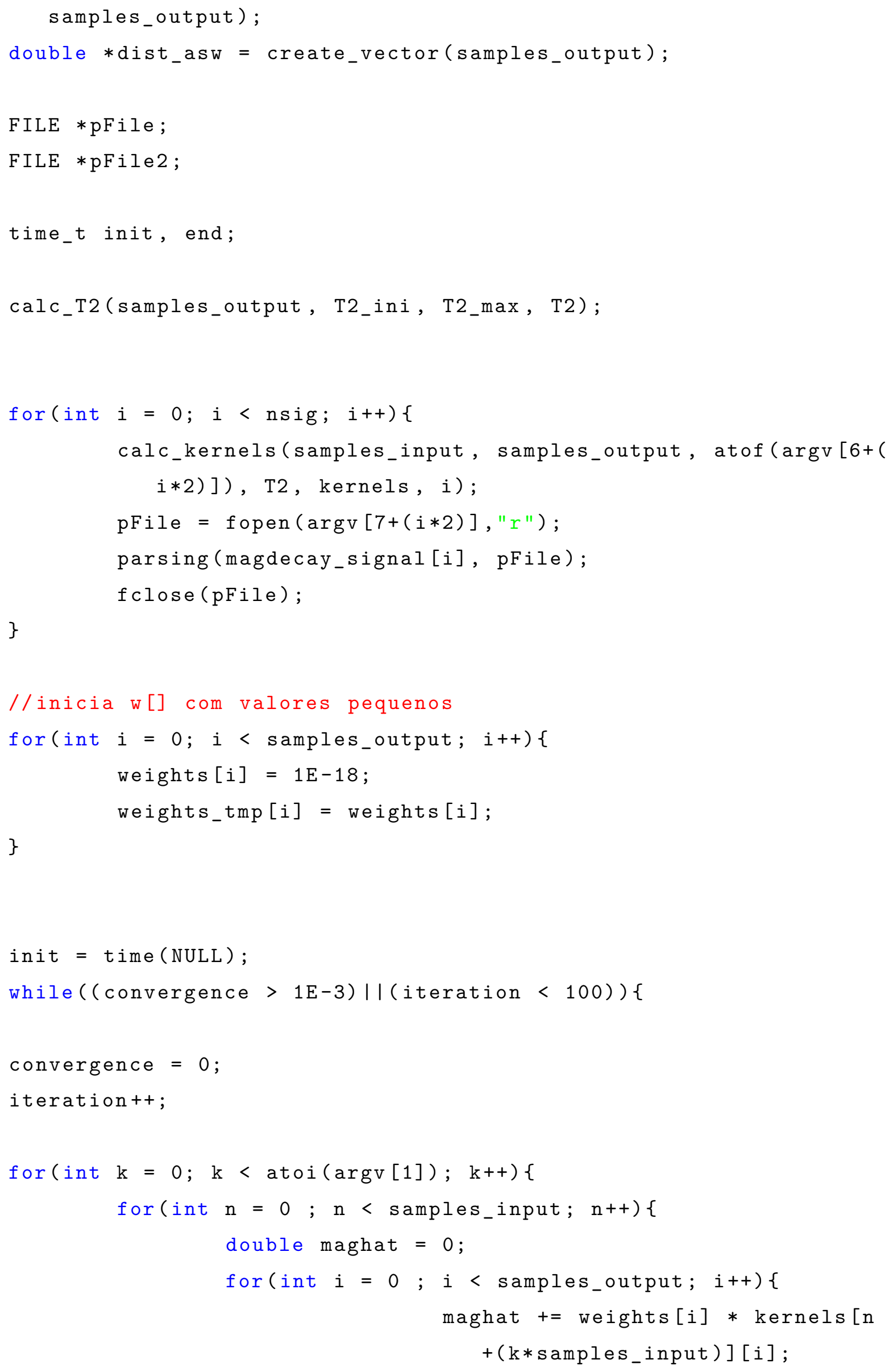




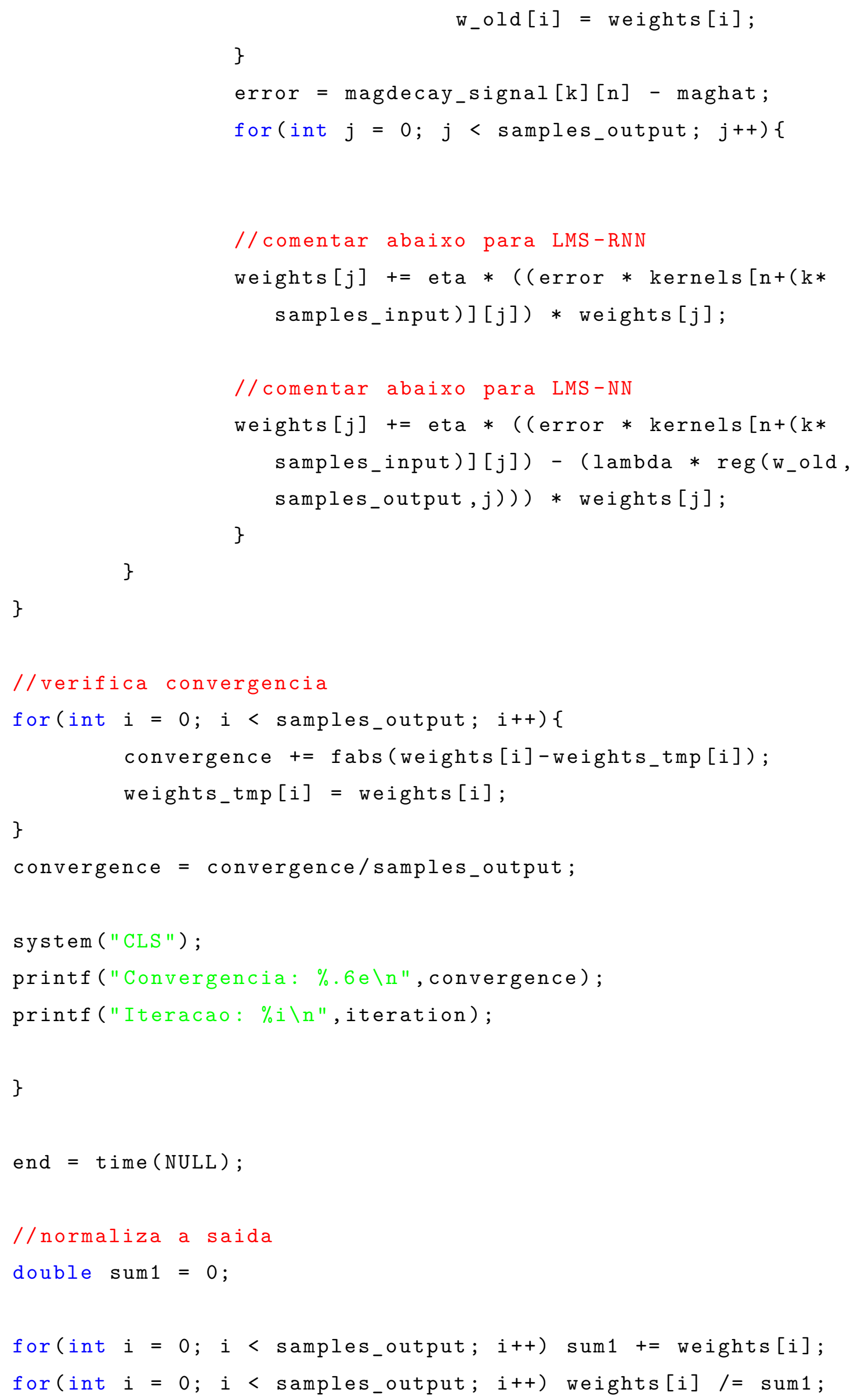




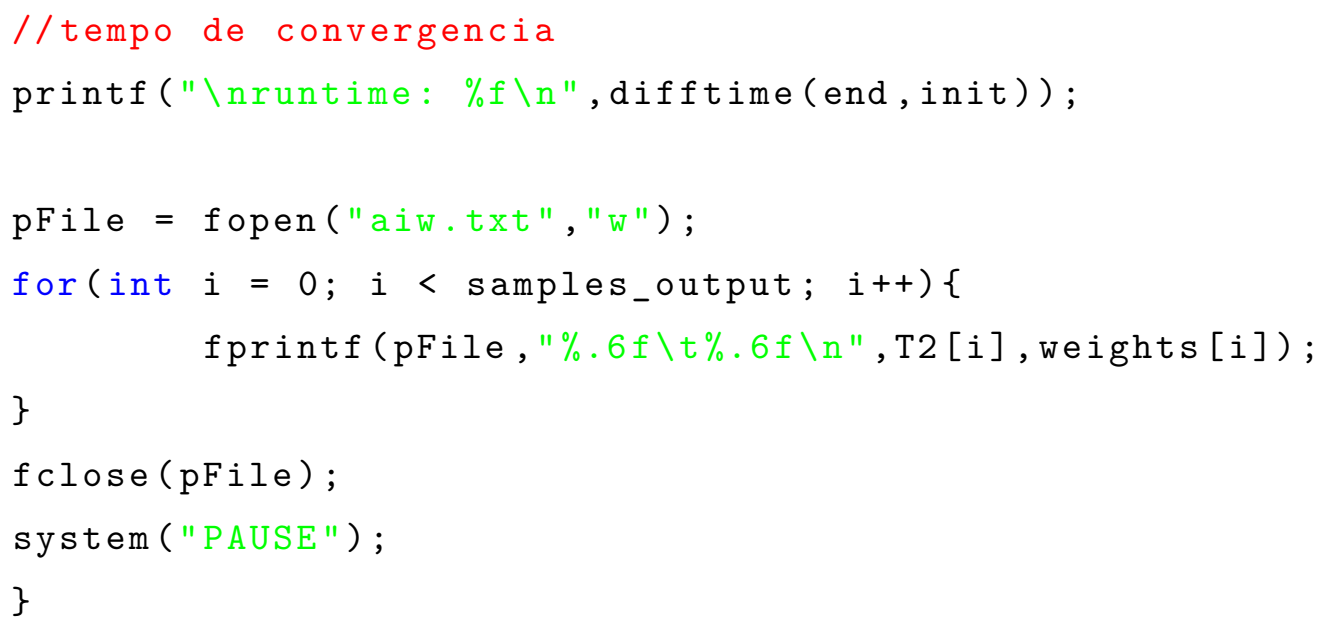


APENNDICE $B$

\section{Código $\mathrm{C}++$ para rede recorrente de Hopfield}

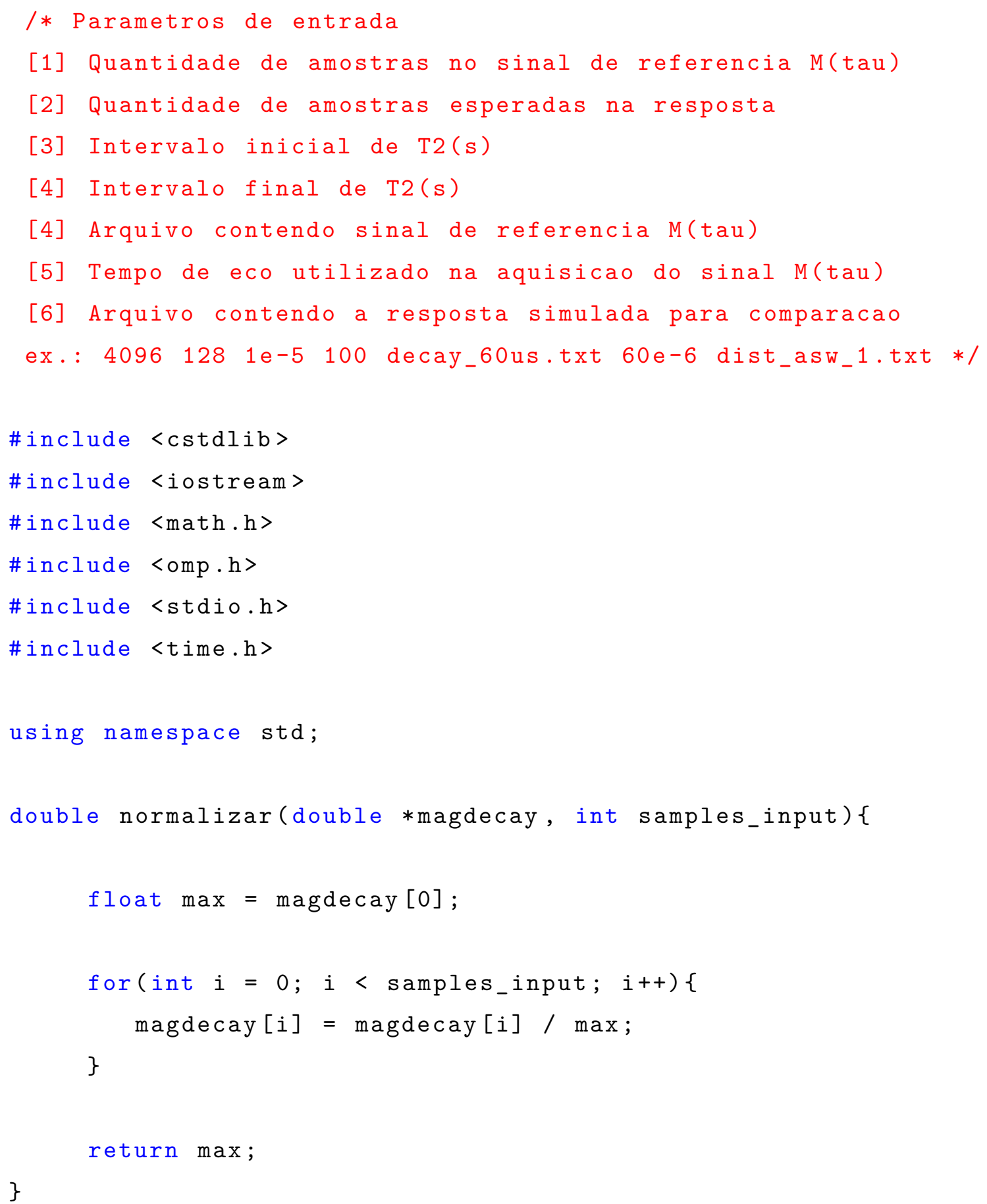




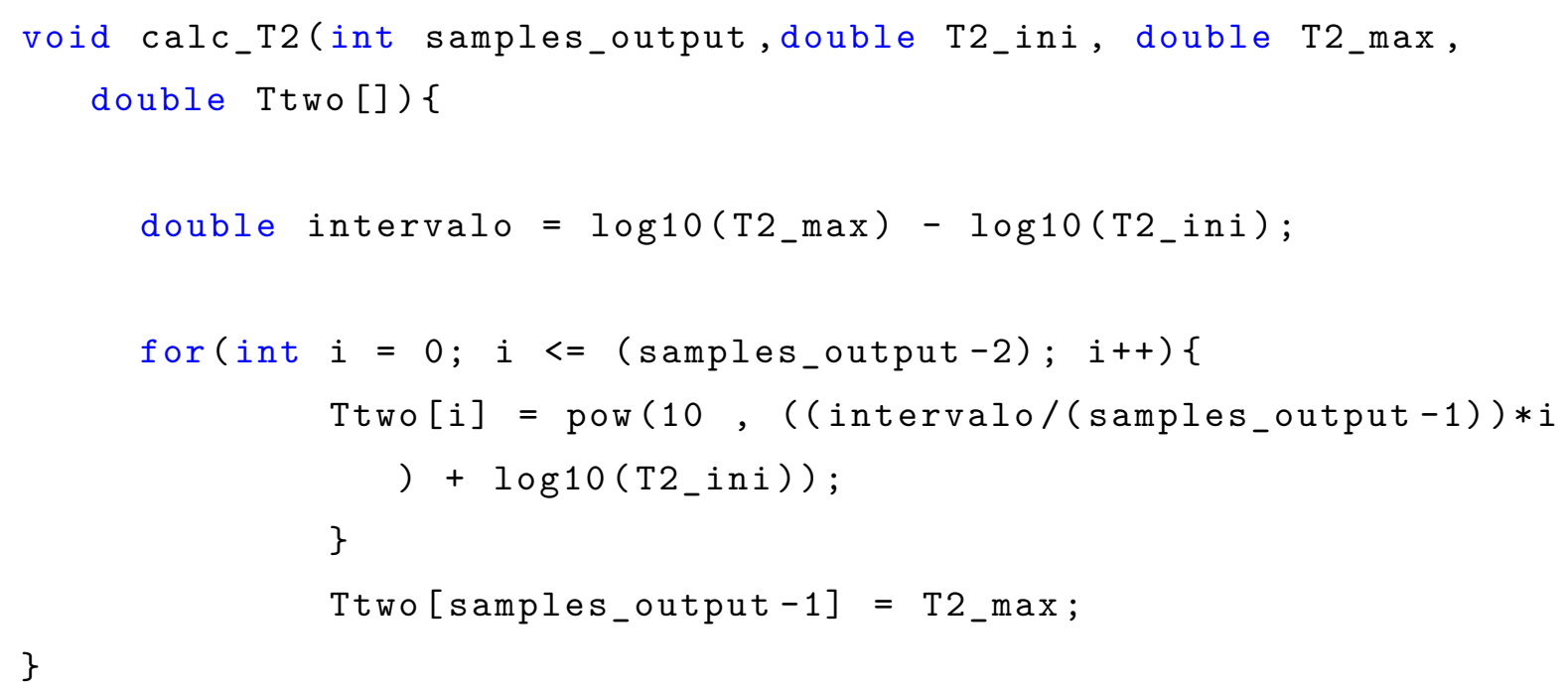




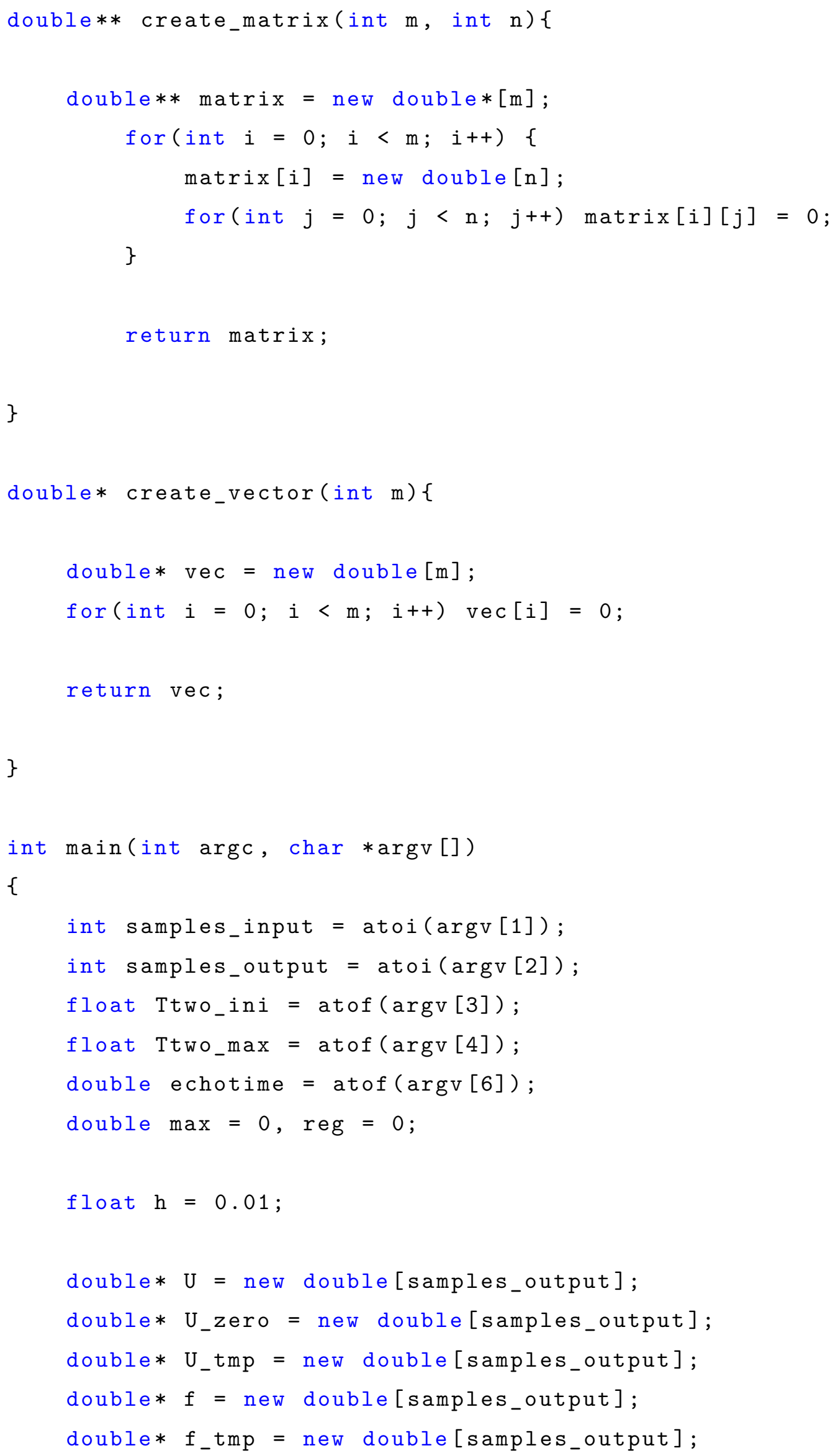




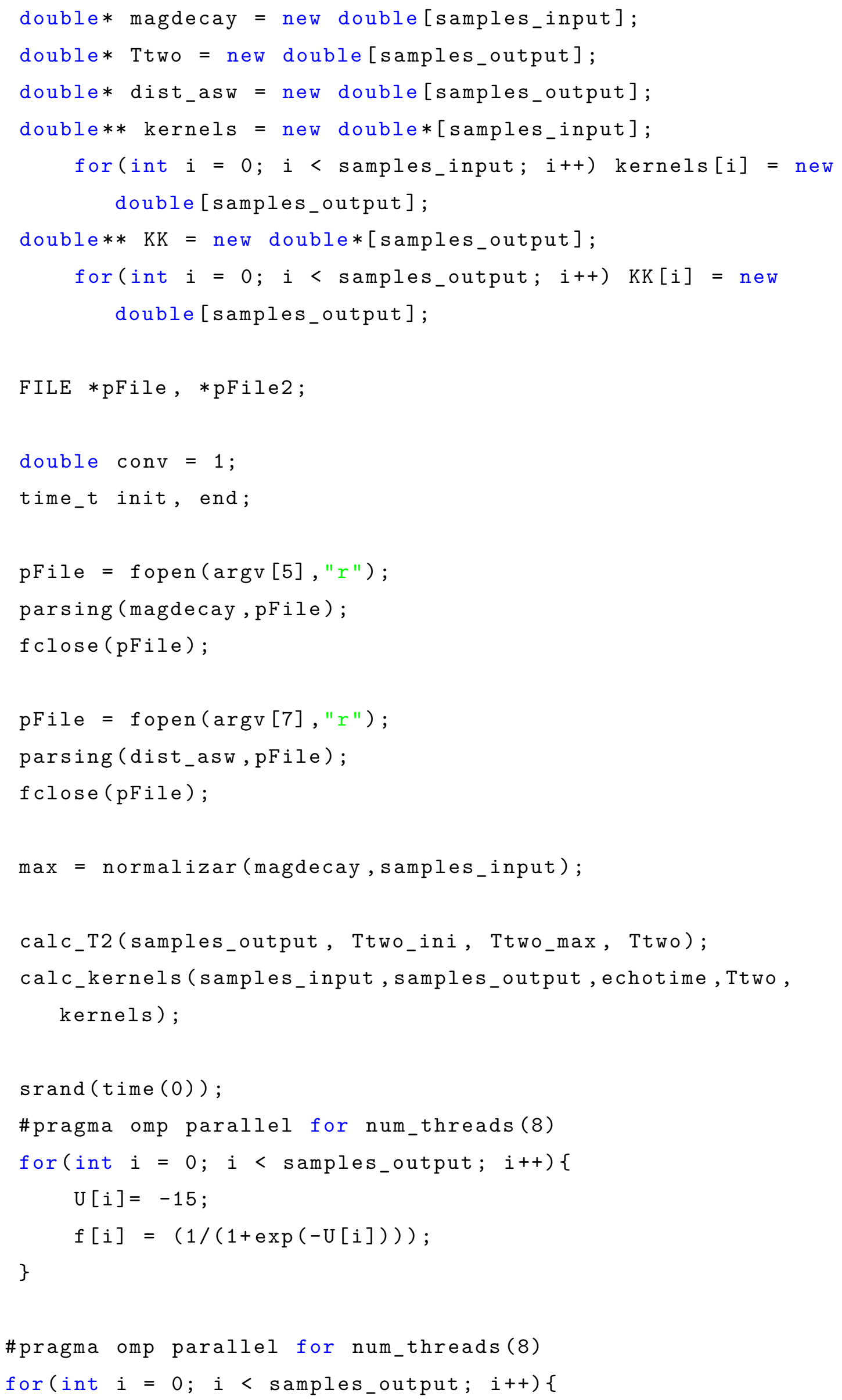




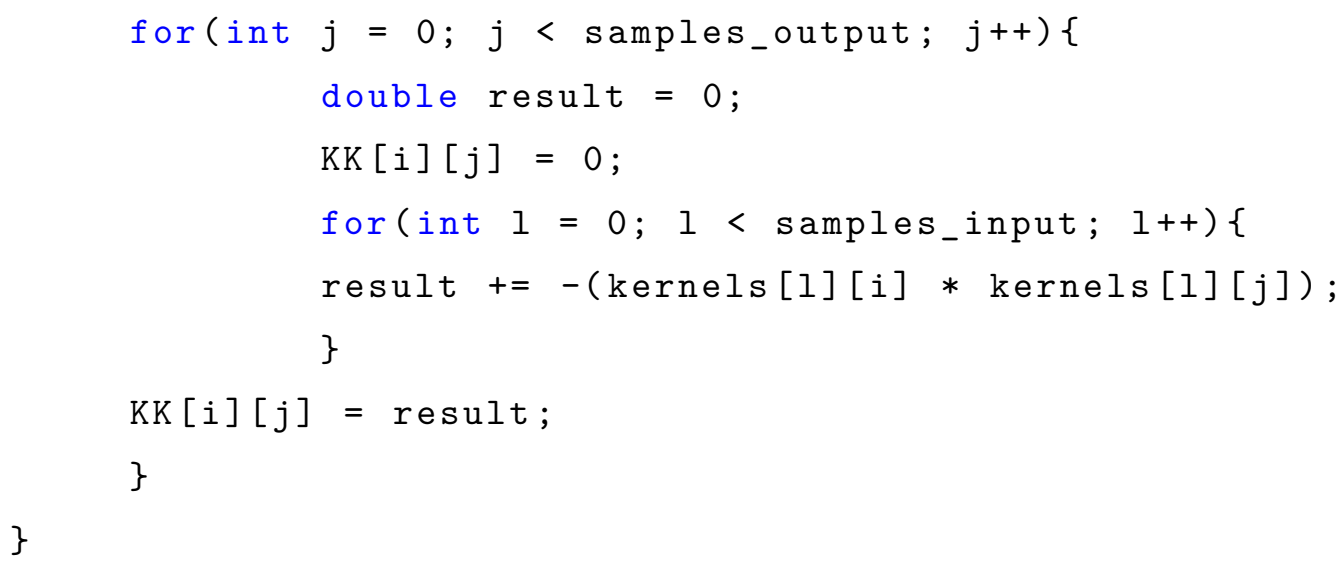




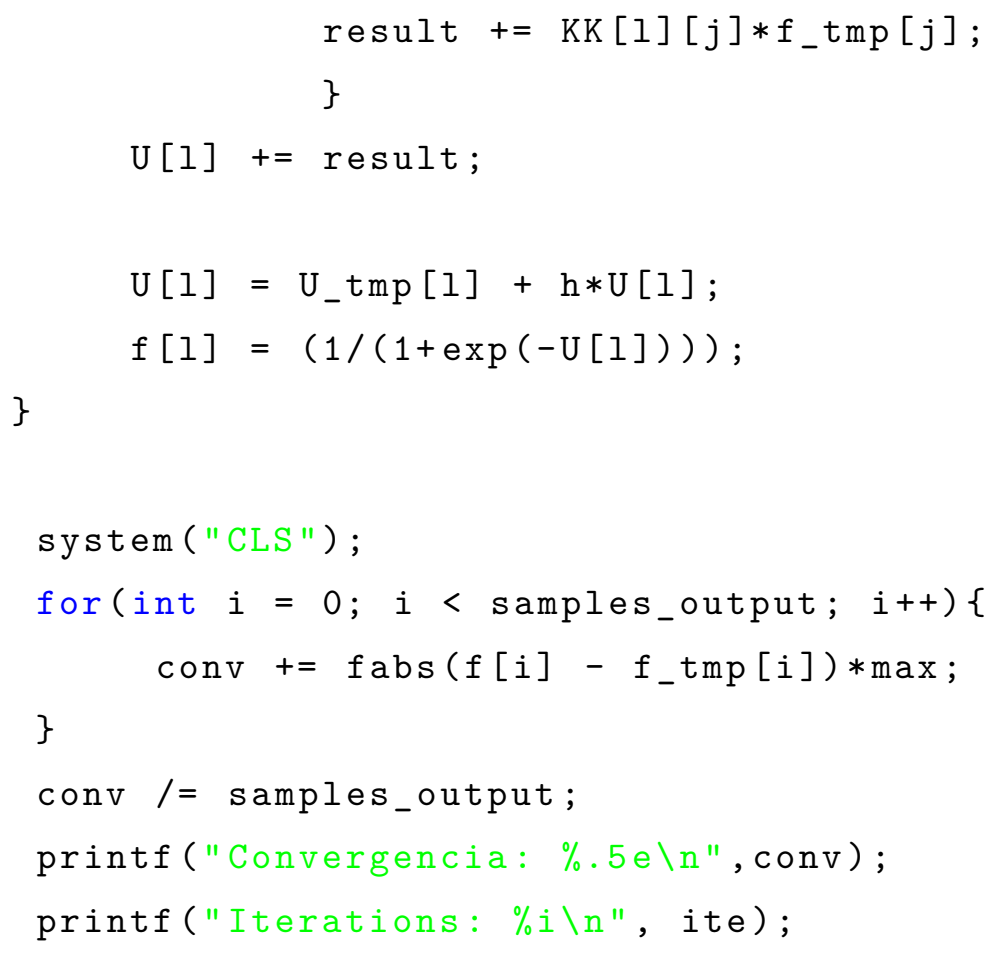




\section{APÊNDICE $C$}

\section{Código $C++$ para $L S-R$}

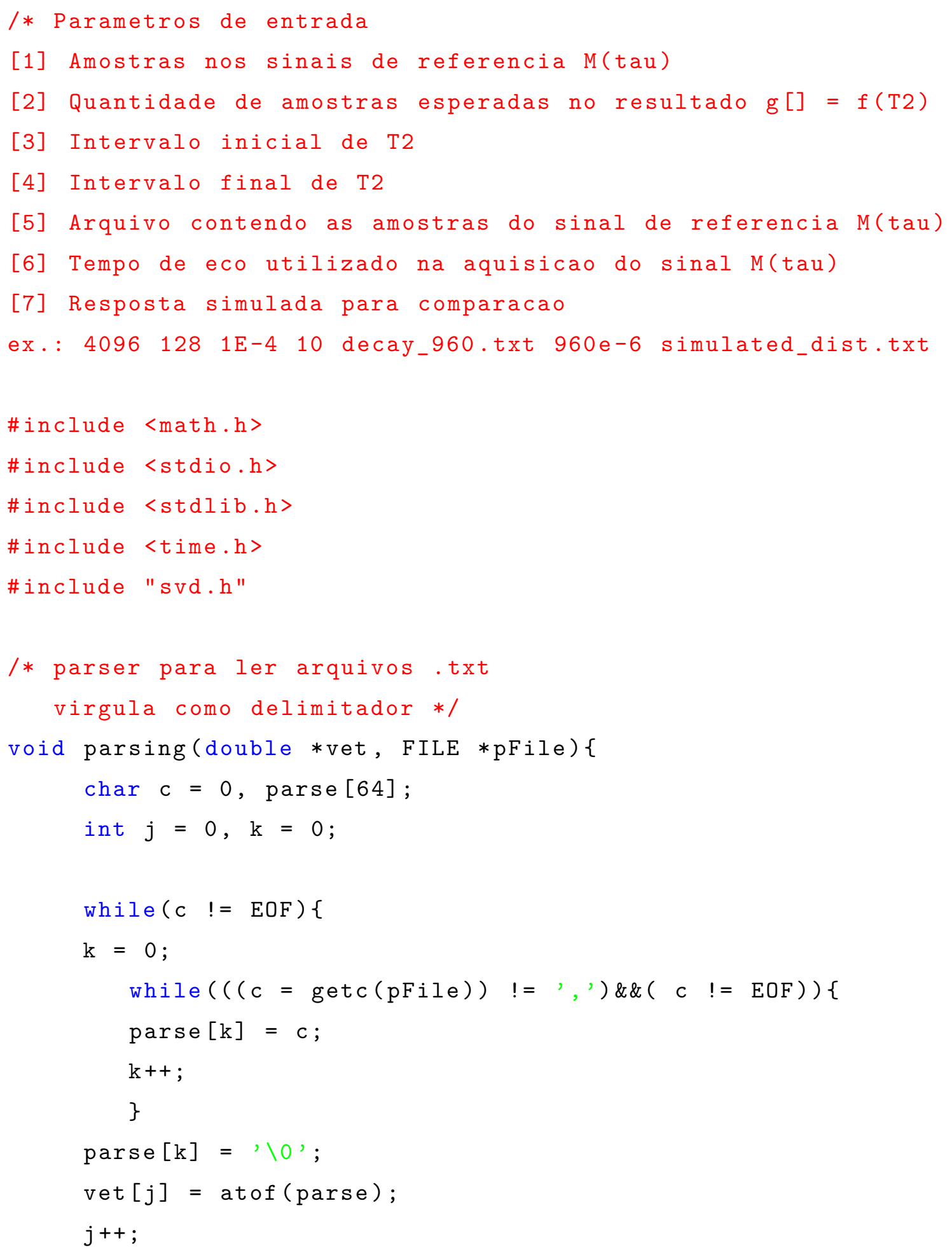




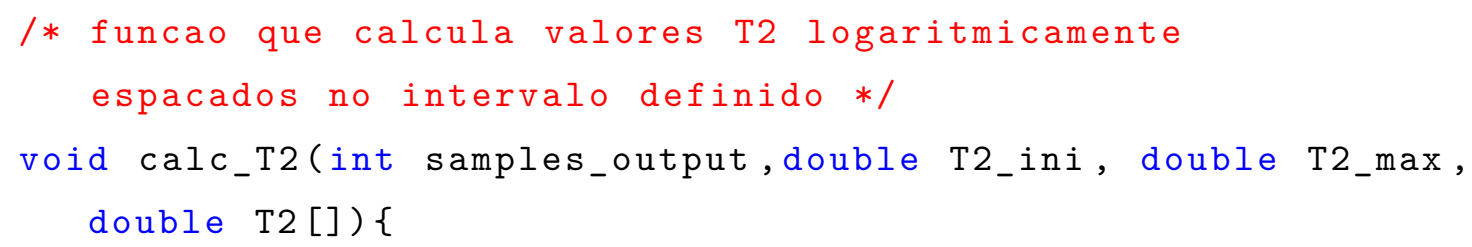




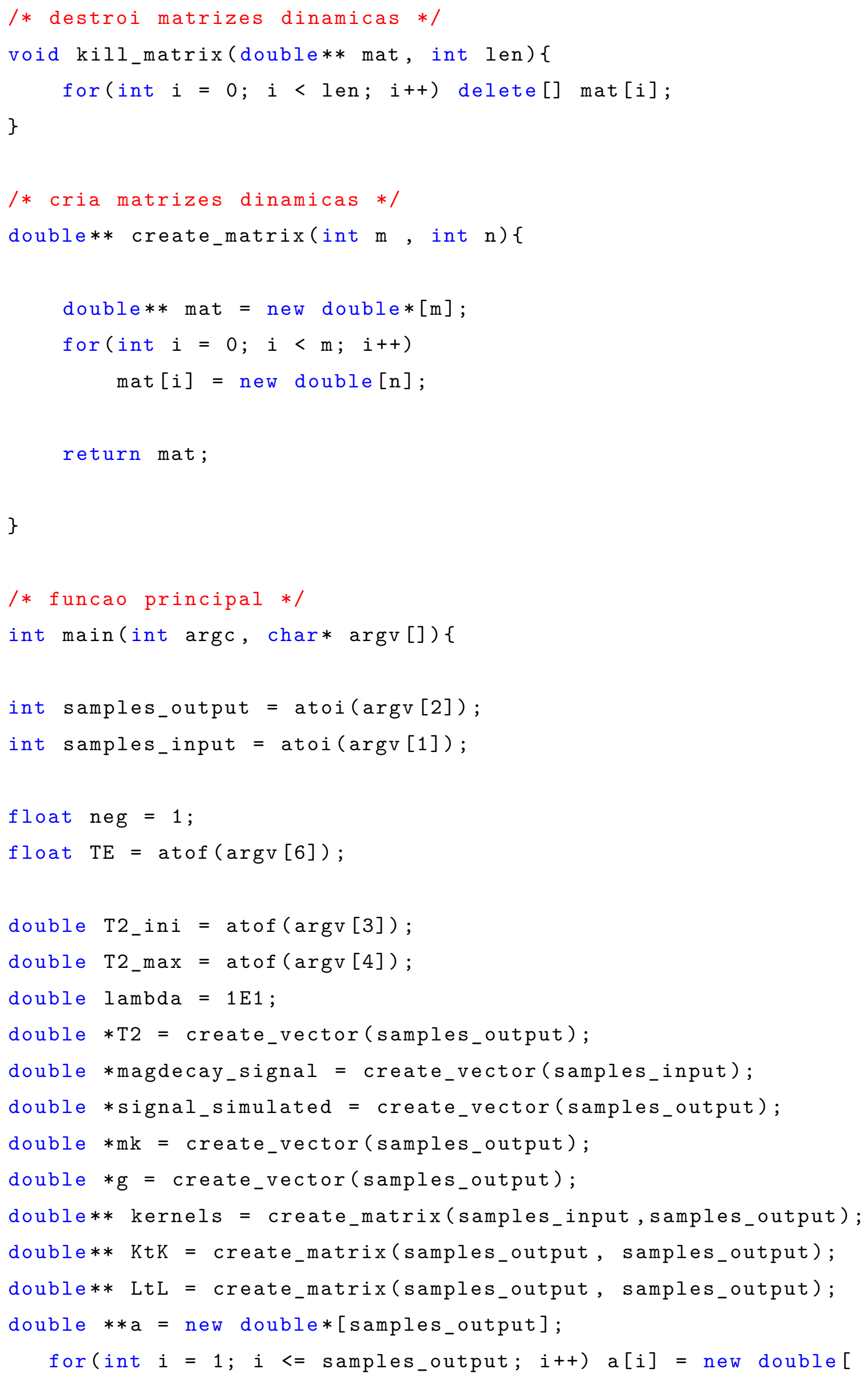


samples_output];

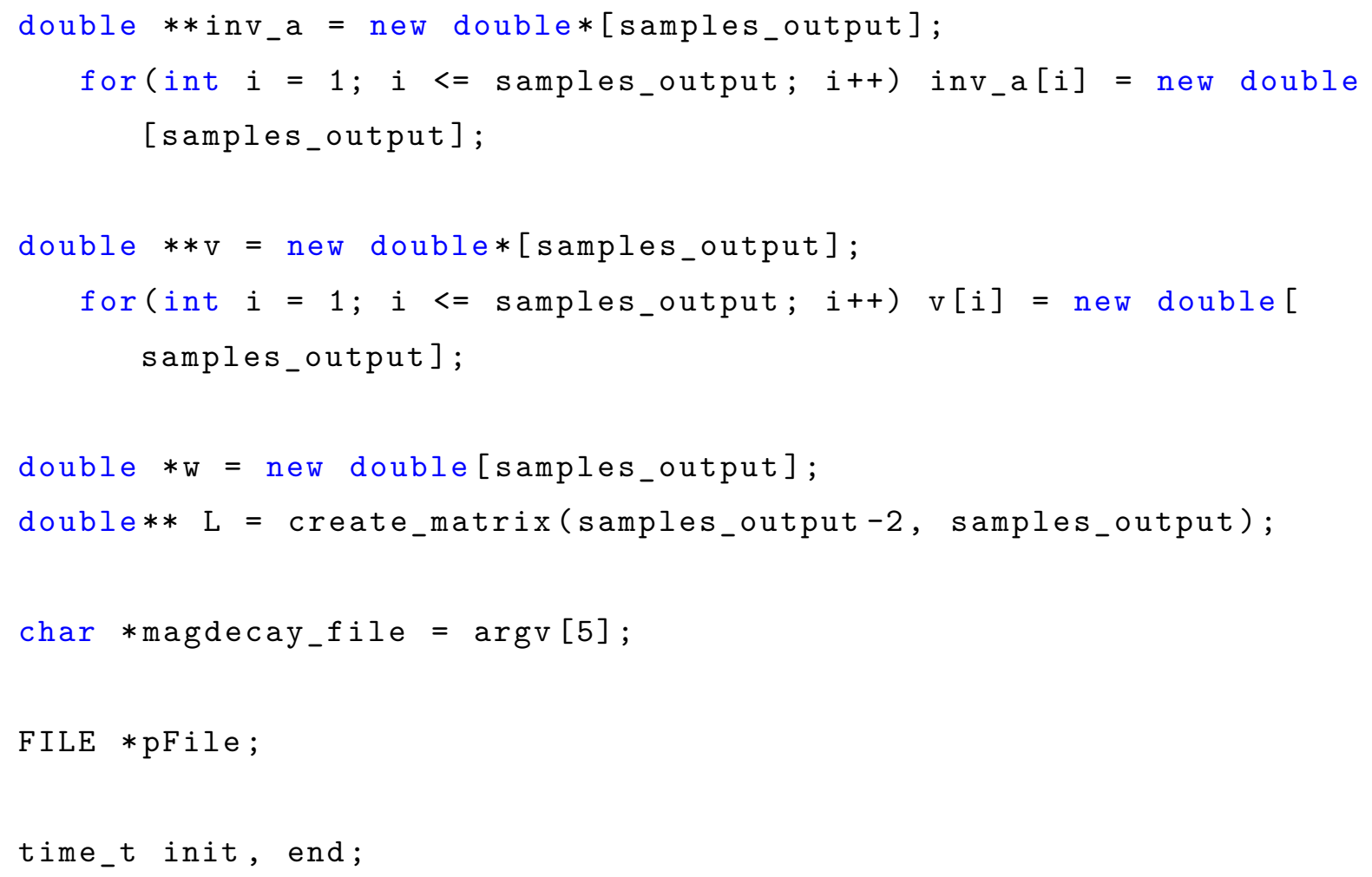




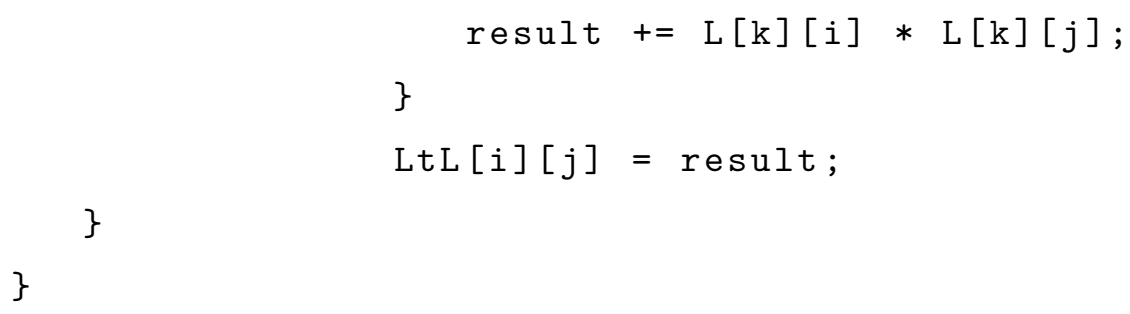




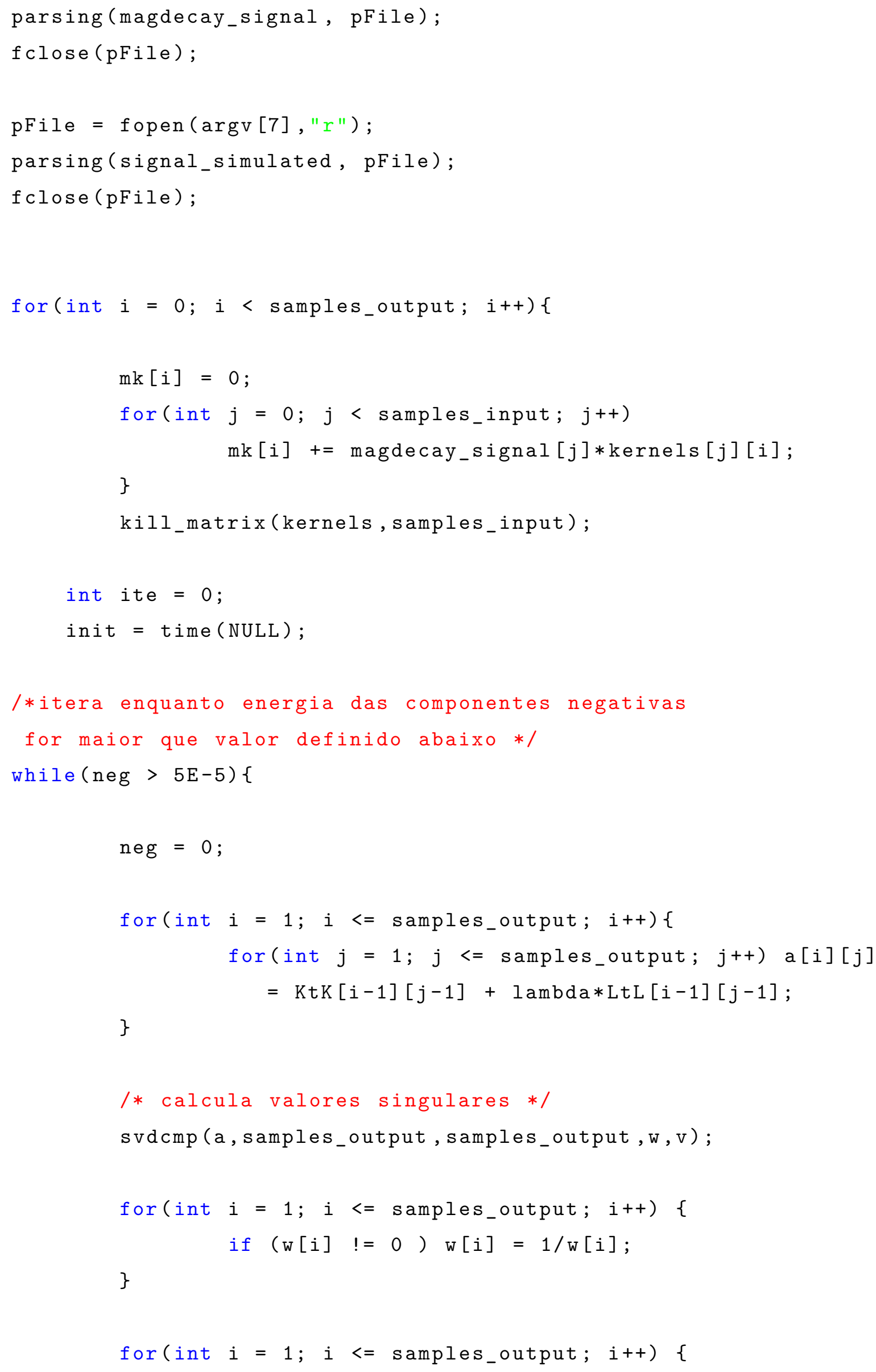




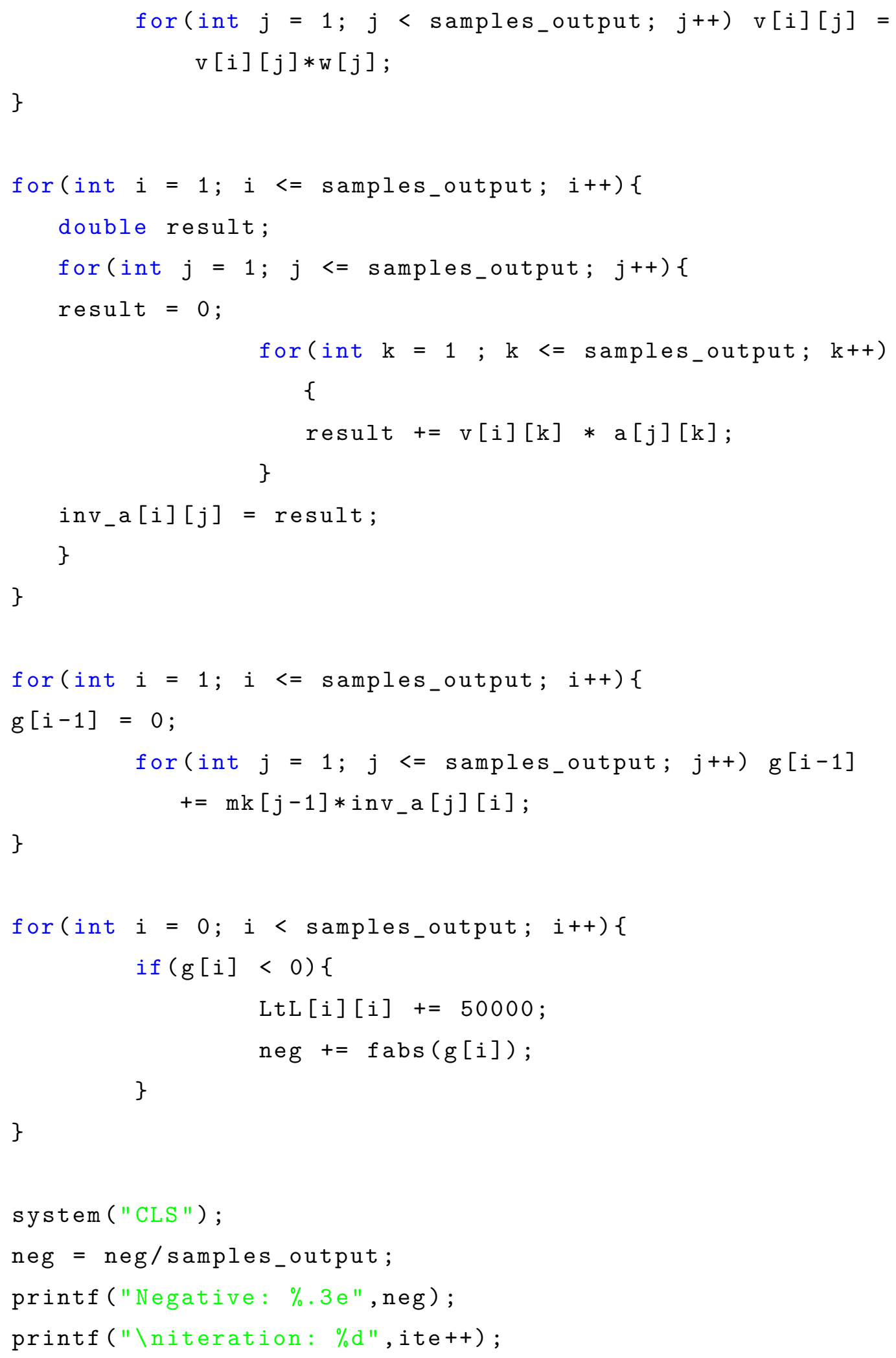




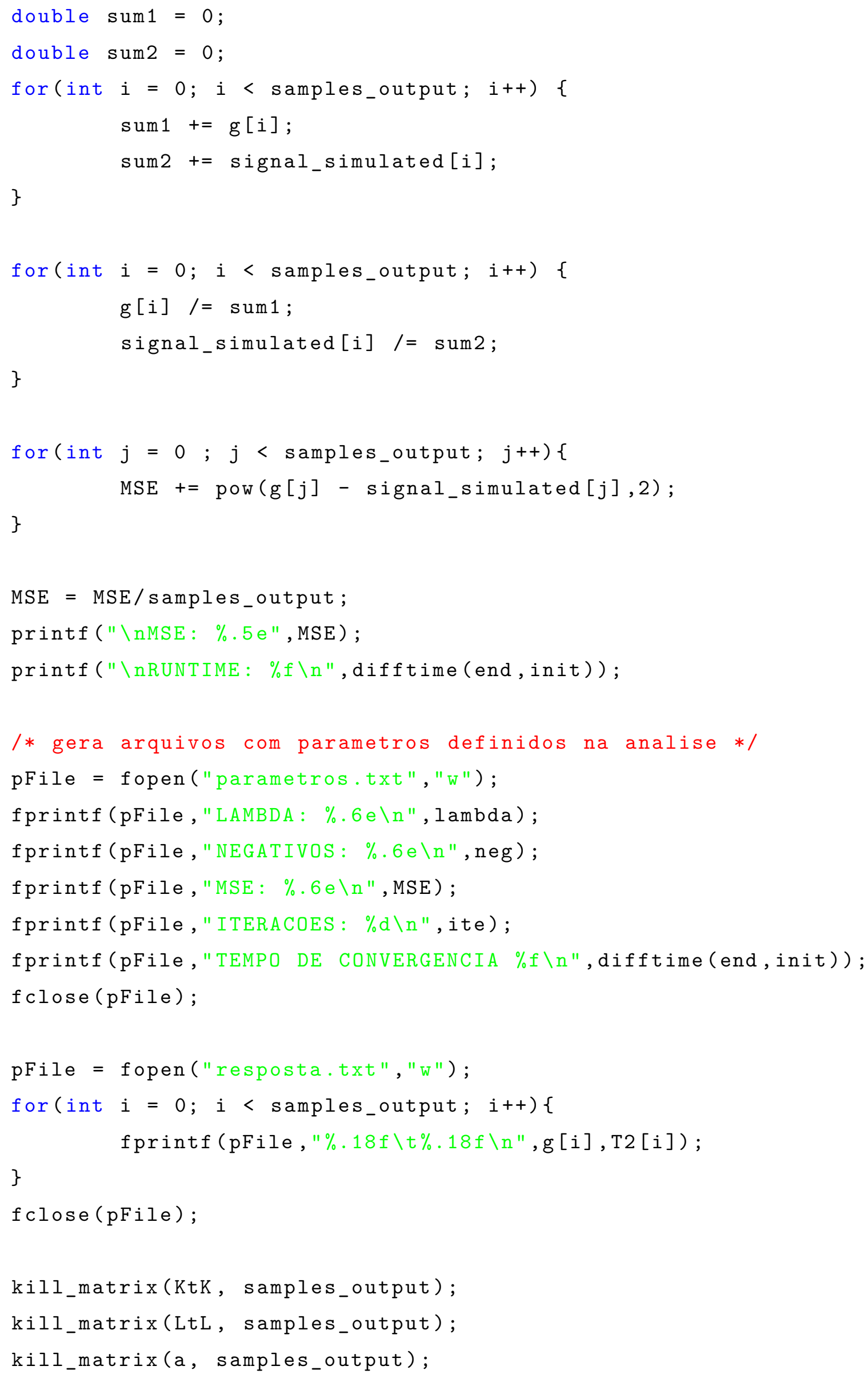




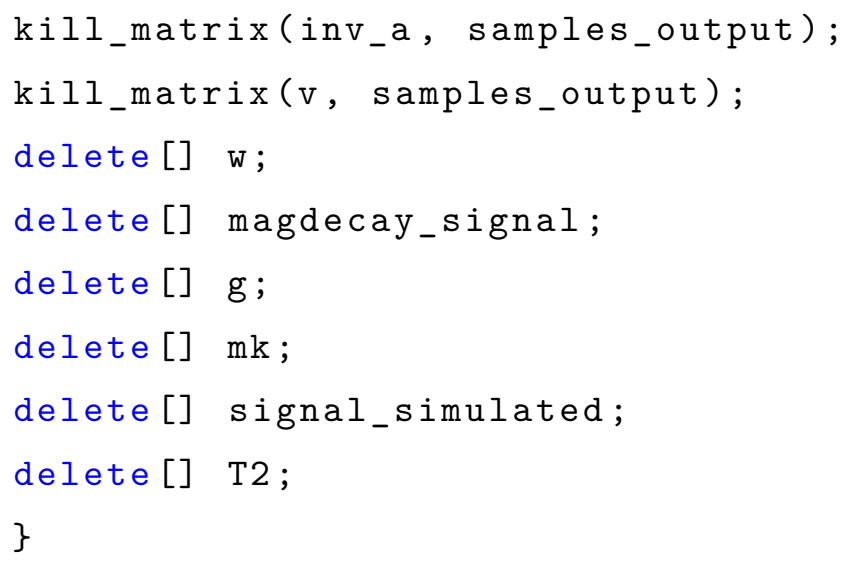

